\title{
Structure-Elucidating Total Synthesis of the (Polyenoyl)tetramic Acid Militarinone C
}

Christian Drescher ${ }^{\dagger}$, Morris Keller + (in part), Olivier Potterat + (in part), Matthias Hamburger* + (in part), Reinhard Brückner*,†

${ }^{\dagger}$ Institut für Organische Chemie

Albert-Ludwigs-Universität Freiburg

Albertstraße 21

79104 Freiburg im Breisgau

Germany

*reinhard.brueckner@organik.chemie.uni-freiburg.de

\$ Pharmazeutische Biologie

Universität Basel

Klingelbergstr. 50

4056 Basel

Switzerland

*matthias.hamburger@unibas.ch 


\section{Table of Contents}

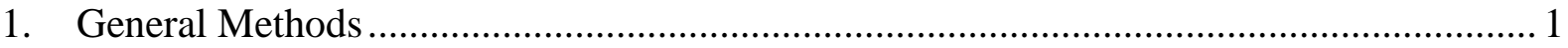

2. Graphical Index for the Experiments and Characterization Data .................................. 3

2.1 Synthesis of the two Enantiomers of Western Block 8 ............................................ 6

2.2 Synthesis of the two Enantiomers of the Eastern Block Precursor 32 ......................... 18

2.3 Synthesis of all 4 Possible Diastereomeres of the Tetramic Acid Militarinone C (1) .. 36

2.4 Isolation and Oxidative Degradation of Natural Militarinone C (1) .......................... 63

2.5 Chiral GLC Studies of the Enantiomeric Alcohol 35 ........................................... 72

2.6 HPLC-Report of the DMB-Tyrosine Ester 13 and the Western Block 8.................... 77

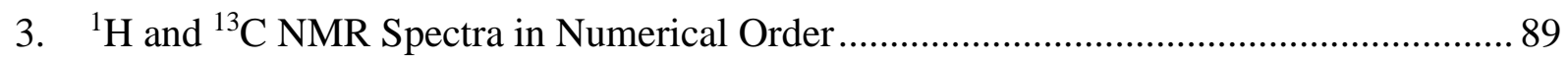

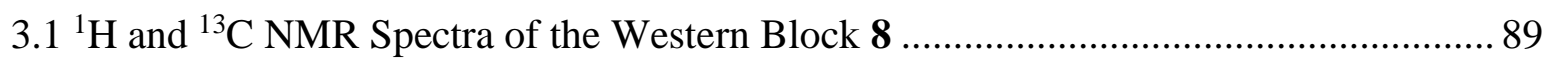

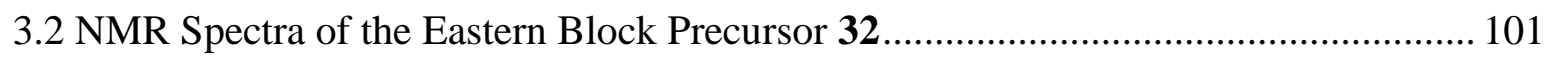

3.3 NMR Spectra of all 4 Diastereomeres of the Tetramic Acid Militarionone C (1)...... 120

3.4 Spectra of the Isolated Products of Paecilomyces militaris RCEF 0095 ..................... 137 


\section{General Methods}

Working technique: All reactions not containing water were carried out under an $\mathrm{N}_{2}$-atmosphere. Reaction flasks were dried in an oven $\left(65^{\circ} \mathrm{C}\right)$ and in vacuo with a heat gun prior to use. Liquids were added with syringe and cannula through a rubber septum. Solids were added in a $\mathrm{N}_{2}$-counterflow. Reactions containing water were carried out without inert gas atmosphere. For reactions that require heating, it was used an oil bath.

Solvents: Tetrahydrofuran (THF) for reactions was distilled over potassium, diethyl ether $\left(\mathrm{Et}_{2} \mathrm{O}\right)$ over sodium-potassium alloy, and dichloromethane $\left(\mathrm{CH}_{2} \mathrm{Cl}_{2}\right)$ and triethyl amine $\left(\mathrm{NEt}_{3}\right)$ over $\mathrm{CaH}_{2}$ under an $\mathrm{N}_{2}$-atmosphere prior to use. Other solvents for that purpose were obtained commercially as "dry" or "extra dry" solvents and used without further purification. Cyclohexane $\left(c \mathrm{C}_{6} \mathrm{H}_{12}\right)$, ethyl acetate $(\mathrm{AcOEt})$, methanol $(\mathrm{MeOH})$, ethanol $(\mathrm{EtOH})$, dichloromethane $\left(\mathrm{CH}_{2} \mathrm{Cl}_{2}\right)$, and tert-butyl methyl ether ( $\left.t \mathrm{BuOMe}\right)$ for workup and column chromatography were distilled using a rotary evaporator prior to use to remove high boiling fractions. Diethyl ether $\left(\mathrm{Et}_{2} \mathrm{O}\right)$, pentane, and chloroform $\left(\mathrm{CHCl}_{3}\right)$ for that purpose were obtained as p. a. grade solvents and used without further purification.

Organo-lithium reagents were stored in a refrigerator in Schlenk flasks with PTFE screw caps and PTFE valves and were titrated using $N$-pivaloyl- $o$-toluidine ${ }^{[1]}$ prior to use.

Chromatography: Thin layer chromatography (TLC) was used to monitor reactions and purification procedures. Merck silica plates with glass as supporting material (TLC Silicagel 60 $\mathrm{F}_{254}$ and TLC Silica gel 60 RP-18 $\mathrm{F}_{254}$ ) were used. Chromatograms were, if applicable, marked in UV light at $254 \mathrm{~nm}$ and subsequently stained using one of the following stains: permanganate stain $\left(0.65 \mathrm{~g} \mathrm{KMnO}_{4}, 3.15 \mathrm{~g} \mathrm{~K}_{2} \mathrm{CO}_{3}, 125 \mathrm{~mL} \mathrm{H}_{2} \mathrm{O}\right)$ or cerium sulfate / molybdophosphoric acid (10 g Ce( $\left(\mathrm{SO}_{4}\right)_{2}, 25 \mathrm{~g}$ molybdophosphoric acid, $1 \mathrm{~L} \mathrm{H}_{2} \mathrm{O} ; 80 \mathrm{~mL}$ conc. $\mathrm{H}_{2} \mathrm{SO}_{4}$ ). Flash column chromatography ${ }^{[2]}$ was conducted on Macherey-Nagel \& Co silica gel $60^{\circledR}$ (230-400 mesh) and Sigma-Aldrich $\mathrm{C}_{18}$-Reversed phase silica gel. Chromatography conditions are documented at the respective experiment in the following manner: (solv1:solv2 $=\mathrm{a}: \mathrm{b} \stackrel{\mathrm{x}}{\longrightarrow} \mathrm{c}: \mathrm{d}, \mathrm{d} \times \mathrm{h} \mathrm{cm}$, $\mathrm{V} \mathrm{mL}$ ), which means: a column with the outer diameter $\mathrm{d} \mathrm{cm}$ is packed with $\mathrm{h} \mathrm{cm}$ silica gel. 
Fractions of the size $\mathrm{V} \mathrm{mL}$ are collected. The product is eluted with the solvents solv1 and solv2 in the ratio a:b. The ratio was changed at fraction $\mathrm{x}$ to $\mathrm{c}: \mathrm{d}$.

Nuclear magnetic resonance spectroscopy: NMR spectra were recorded by Dr. M. Keller, F. Reinbold, and M. Schonhard on a Bruker Avance 400 spectrometer $\left[{ }^{1} \mathrm{H}(400 \mathrm{MHz}),{ }^{13} \mathrm{C}\right.$ (100 MHz), DQF-COSY, edHSQC, and HMBC experiments] and a Bruker DRX 500 spectrometer $\left[{ }^{1} \mathrm{H}(500 \mathrm{MHz}),{ }^{13} \mathrm{C}(126 \mathrm{MHz}), \mathrm{DQF}-\mathrm{COSY}\right.$, edHSQC, and HMBC experiments] or by myself on a Varian Mercury VX 300 spectrometer $\left[{ }^{1} \mathrm{H}(300 \mathrm{MHz})\right]$ or on a Bruker Avance 300 spectrometer $\left[{ }^{1} \mathrm{H}(300 \mathrm{MHz})\right] .{ }^{1} \mathrm{H}$ NMR spectra were referenced internally to TMS or the solvent signal respectively $\left(\mathrm{CDCl}_{3}: 7.26 \mathrm{ppm}, \mathrm{C}_{6} \mathrm{D}_{6}: 7.16 \mathrm{ppm}, \mathrm{MeOD}: 3.30 \mathrm{ppm}, \mathrm{D}_{2} \mathrm{O}: 4.79\right.$ $\mathrm{ppm}) .{ }^{13} \mathrm{C}$ NMR spectra were referenced internally to the solvent signal $\left(\mathrm{CDCl}_{3}: 77.10 \mathrm{ppm}\right.$, $\mathrm{C}_{6} \mathrm{D}_{6}$ : $\left.128.00 \mathrm{ppm}, \mathrm{MeOD}: 49.00 \mathrm{ppm}\right) .{ }^{1} \mathrm{H}$ NMR data are reported as follows: chemical shift ( $\delta$ in $\mathrm{ppm}$ ), multiplicity ( $\mathrm{s}$ for singlet; $\mathrm{d}$ for doublet; $\mathrm{t}$ for triplet; $\mathrm{m}$ for multiplet; $\mathrm{m}_{\mathrm{c}}$ for symmetrical multiplet; br for broad signal), coupling constant(s) (Hz; ${ }^{3} J$ couplings unless otherwise noted), integral, and specific assignment. ${ }^{13} \mathrm{C} \mathrm{NMR}$ data are reported in terms of chemical shift and assignment. For AB signals the high-field part was named A and the lowfield part B.

High resolution mass spectrometry: High resolution mass spectra were recorded by Dr. J. Wörth and C. Warth on a Thermo Scientific Exactive mass spectrometer equipped with an orbitrap (ion trap) analyzer. Ionization method: Electrospray ionization (ESI; spray voltage: 2.5-4.0 kV) or atmospheric pressure chemical ionization (APCI; spray current: $5 \mu \mathrm{A}$ ).

Melting points: Melting points were determined in a Büchi melting point apparatus using open glass capillaries.

IR spectroscopy: IR spectra were recorded on a Perkin Elmer Paragon 1000 FT-IR spectrometer for a film of the substance on a $\mathrm{NaCl}$ plate unless otherwise stated.

Specific rotations: Optical rotations were measured using a 341 MC Perkin-Elmer polarimeter. The specific rotations $[\alpha]_{\lambda}^{\mathrm{T}}$ were calculated by the formula:

$$
[\alpha]_{\lambda}^{\mathrm{T}}=\frac{\alpha_{\text {observed }} \times 100}{c \times d}
$$

with $\mathrm{T}=$ temperature in ${ }^{\circ} \mathrm{C}, \alpha_{\mathrm{observed}}=$ the experimentally observed optical rotation, $c=$ concentration in $\mathrm{g} / 100 \mathrm{~mL}, d=$ length of the cuvette in $\mathrm{dm}$. 


\section{Graphical Index for the Experiments and Characterization Data}

Content of Section 2.1 (pp. 6): Synthesis of the two Enantiomers of Western Block 8:
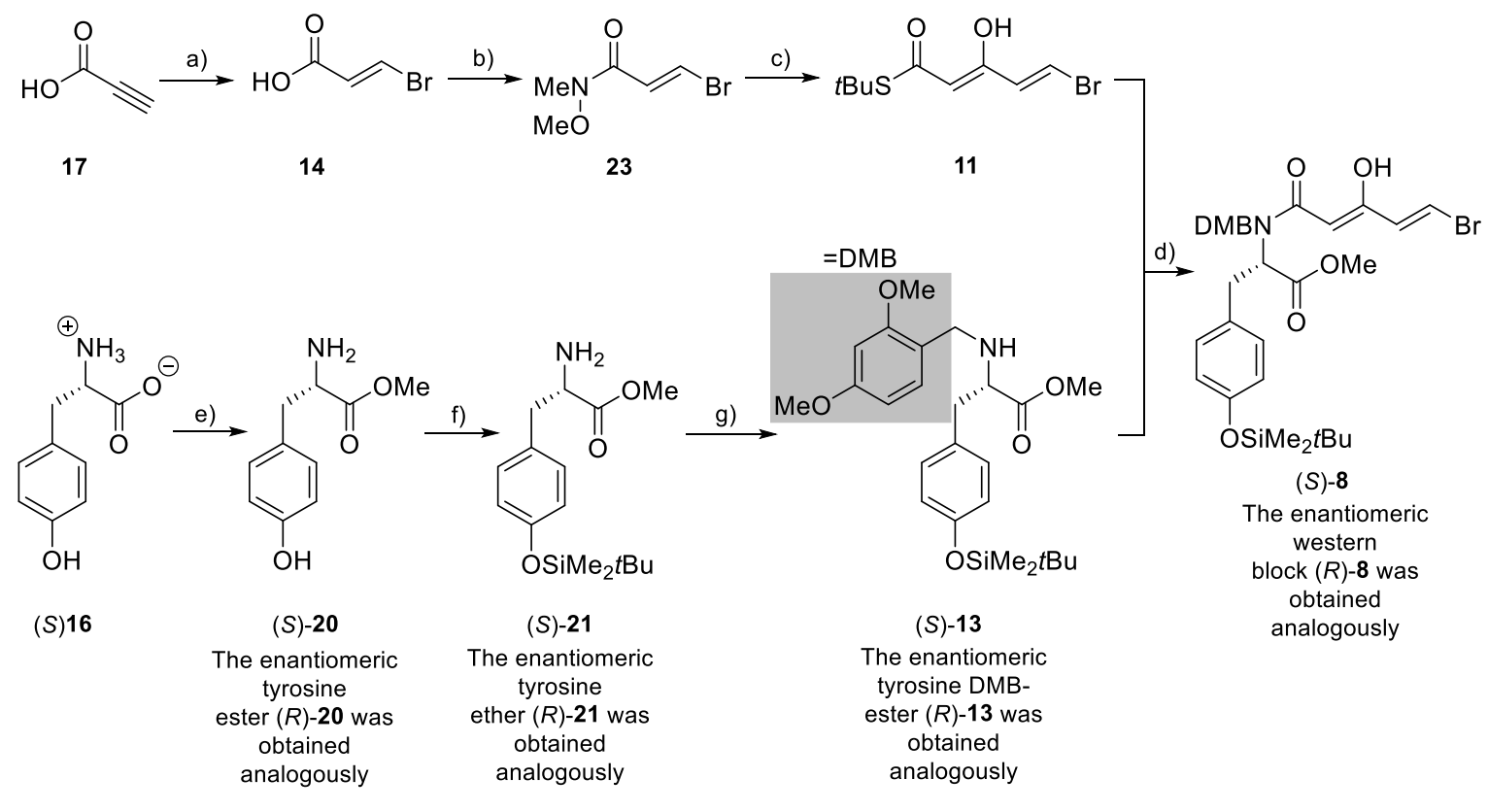

Content of Section 2.2 (pp. 18): Synthesis of the two Enantiomers of the Eastern Block

Precursor 32:

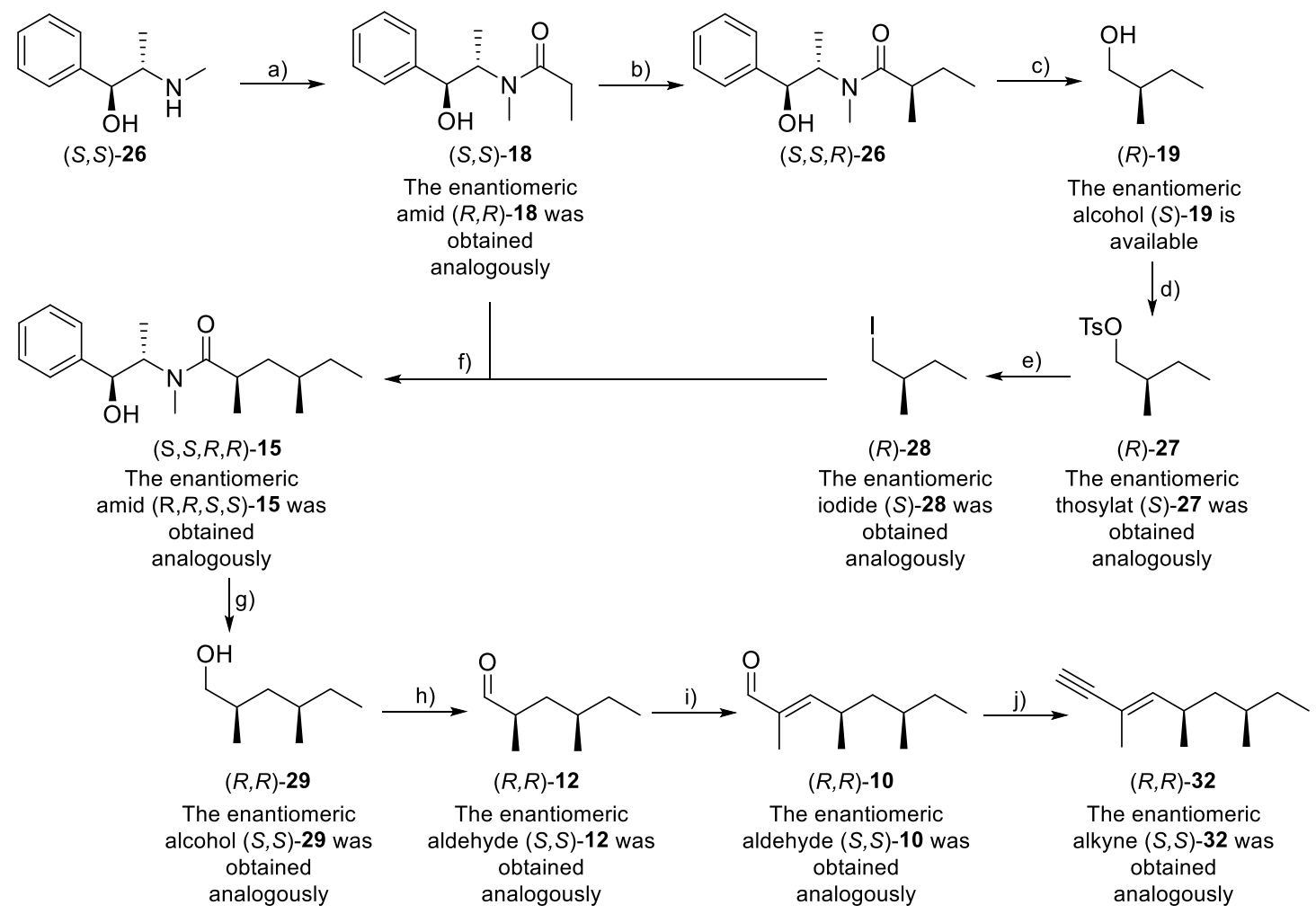




\section{Content of Section 2.3 (pp. 36): Synthesis of all 4 Possible Diastereomeres of the Tetramic}

\section{Acid Militarinone C (1):}

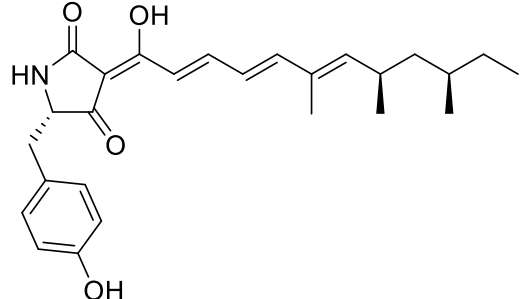

$(S, R, R)-1$

The enantiomeric

tetramic acid $(R, S, S)-1$ was

obtained

analogously
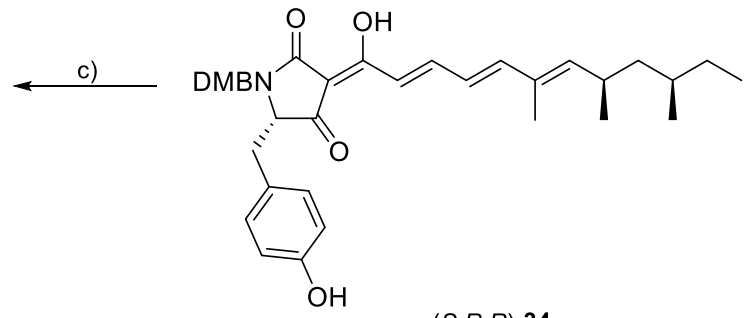

$(S, R, R)-34$

The enantiomeric

tetramic acid $(R, S, S)$-34 was

obtained

analogously

b)<smiles>COC(=O)[C@H](Cc1ccc(OCC(C)(C)C)cc1)N(C(=O)O)C(=O)C=C(O)C=CBr</smiles>

(S)-8<smiles>C#CC(C)=CC(C)CC(C)CC</smiles>

$(R, R)-32$

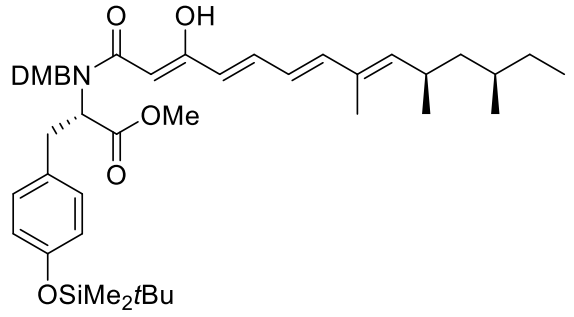

$(S, R, R)-33$

The enantiomeric polyene $(R, S, S)-33$ was obtained analogously<smiles>CCCCOC(=O)[C@@H](C(=O)/C=C(O)/C=C/Br)[C@H](Cc1ccc(OCCCC)cc1)C(=O)OC</smiles>

(S)-8<smiles>C#CC(C)=C[C@@H](C)C[C@H](C)CC</smiles>

$(S, S)-32$<smiles>CCOc1ccc(C[C@@H](C(=O)OC)N(C(=O)/C=C(O)/C=C/C=C/C(C)=C/[C@@H](C)CC(C)CC)C(C)(C)C)cc1</smiles>

$(S, S, S)-33$

The enantiomeric polyene $(R, R, R)-33$ was

obtained

analogously

b)

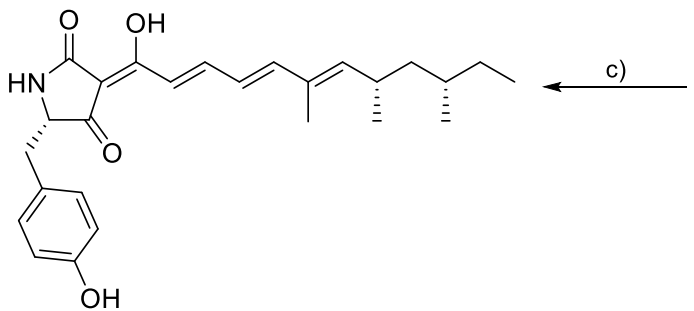

$(S, S, S)-1$

The enantiomeric

tetramic acid $(R, R, R)-1$ was

obtained

analogously

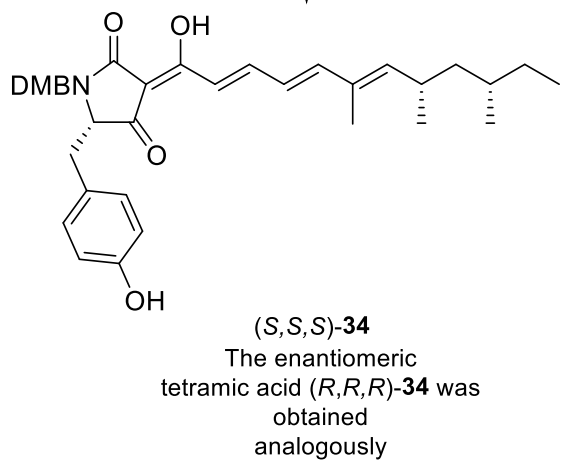


Content of Section 2.4 (pp. 63): Isolation and Oxidative Degradation of Natural Militarinone C (1):

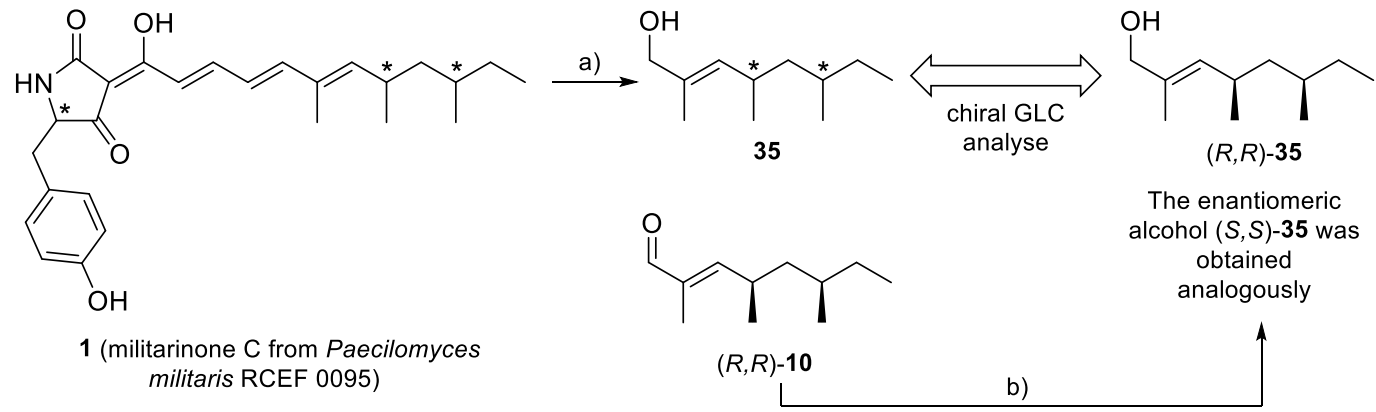

Content of Section 2.5 (pp. 72): Chiral GC Studies of the Enantiomeric Alcohol 35

Content of Section 2.6 (pp. 77): HPLC-Report of the DMB-Tyrosine Ester 13 and the Western Block 8

Reproductions of chromatograms are shown 


\subsection{Synthesis of the two Enantiomers of Western Block 8}

trans-3-Bromacrylic Acid (14)<smiles>O=C(O)C=CBr</smiles>

14

A mixture of propiolic acid (23) $(6.02 \mathrm{~g}, 86.0 \mathrm{mmol})$ and aq. $\mathrm{HBr}$ solution $(8.9 \mathrm{M}, 35 \mathrm{~mL})$ was heated to reflux for $1.5 \mathrm{~h}$ and cooled to room temperature. Further cooling of the mixture to $0^{\circ} \mathrm{C}$ resulted in the formation of crystals, which were collected by filtration, washed with $\mathrm{H}_{2} \mathrm{O}(50$ $\mathrm{mL}$ ), and dried to leave the product $14\left[9.67 \mathrm{~g}, 75 \%, 64.5 \mathrm{mmol}\left(\right.\right.$ Lit. $\left.\left.^{3}: 85 \%\right)\right]$ as brown needles.

$\mathbf{R}_{\mathbf{f}}\left(c \mathrm{C}_{6} \mathrm{H}_{12}: \mathrm{AcOEt}=4: 1\right)=0.35$

${ }^{1} \mathbf{H}$ NMR (300.13 MHz, $\left.\mathrm{CDCl}_{3}, \mathrm{DsBrAu} 18-30540,18.8 .2016\right): \delta=6.54\left(\mathrm{~d}, J_{2,3}=14.0 \mathrm{~Hz}, 1 \mathrm{H}\right.$, 2-H), $7.76\left(\mathrm{~d}, J_{3,2}=14.0 \mathrm{~Hz}, 1 \mathrm{H}, 3-\mathrm{H}\right), 8.56$ (br. s, 1H, 1-OH).

\section{$N$-Methylmethoxyamine (SI-1)}<smiles>CNOC</smiles>

\section{SI-1}

A solution of $N$-methylmethoxyamine hydrochloride $(6.05 \mathrm{~g}, 60.0 \mathrm{mmol})$ in 2-propanole: $\mathrm{H}_{2} \mathrm{O}$ $=(1: 1,20 \mathrm{~mL})$ was treated with $\mathrm{KOH}(85$ wt. $-\%, 3.60 \mathrm{~g}, 54.5 \mathrm{mmol}, 0.9$ eq. $)$ at $-10^{\circ} \mathrm{C}$. The mixture has been distillated (bp. 1013 mbar: $45-50^{\circ} \mathrm{C}$ ). The product $\left[1.52 \mathrm{~g}, 40 \%, 24.0 \mathrm{mmol}\left(\mathrm{Lit} .{ }^{4}\right.\right.$ : $60 \%)$ ] was obtained as a colorless liquid.

${ }^{1} \mathbf{H}$ NMR $\left(300.13 \mathrm{MHz}, \mathrm{CDCl}_{3} / \mathrm{CHCl}_{3}\right.$, sample contained 7 weight-\% of isopropanol with $\mathrm{d}$ at 1.20 and sep at 4.02, DsBrAp24-30930, 24.4.2018): $\delta=2.72\left(\mathrm{~s}, 3 \mathrm{H}, \mathrm{N}-\mathrm{CH}_{3}\right), 3.53(\mathrm{~s}, 3 \mathrm{H}, O-$ $\mathrm{CH}_{3}$ ), 5.52 (br. s, $1 \mathrm{H}, N \mathrm{H}$ ).

\footnotetext{
${ }^{3}$ Y. Luo, I. D. Roy, A.G. E. Madec, H. W. Lam, Angew. Chem. Int. Ed. 2014, 53, 4186-4190.

${ }^{4}$ P. Beak, A. Basha, B. Kokko, D. Loo, J., Am. Chem. Soc. 1986, 108, 6016-6023.
} 


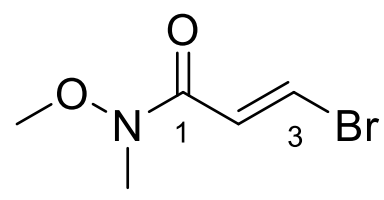

23

At $0^{\circ} \mathrm{C}$ a solution of propylphosphoric acid anhydride in $\mathrm{CH}_{2} \mathrm{Cl}_{2}(54$ wt.- $\%, 0.42 \mathrm{~mL}, 0.56 \mathrm{~g}$, $0.88 \mathrm{mmol}, 1.2$ eq.) was added dropwise to a solution of $N, O$-dimethylhydroxylamine SI-1 (60 mg, $0.88 \mathrm{mmol}$, $1.2 \mathrm{eq}$.$) , trans-3-bromoacrylic acid (14) (120 \mathrm{mg}, 0.80 \mathrm{mmol})$, and $\mathrm{N}$ methylmorpholine (180 $\mu \mathrm{L}, 160 \mathrm{mg} 1.60 \mathrm{mmol}, 2.0$ eq.) in $\mathrm{CH}_{2} \mathrm{Cl}_{2}(9 \mathrm{~mL})$. After $30 \mathrm{~min}$ the solution was diluted with $\mathrm{H}_{2} \mathrm{O}(3 \mathrm{~mL})$ und $\mathrm{HOAc}(\mathrm{pH}=3-4)$. The layers were separated and the aq. layer was extracted with $\mathrm{CH}_{2} \mathrm{Cl}_{2}(3 \times 5 \mathrm{~mL})$. The combined organic layers were dried over $\mathrm{Na}_{2} \mathrm{SO}_{4}$ and concentrated under reduced pressure. Purification by flash chromatography (PE 30/50: $\mathrm{Et}_{2} \mathrm{O}=3: 1,1.5 \mathrm{~cm}, 10 \mathrm{~cm}, 8 \mathrm{~mL}$ ) afforded 23 [134 mg, 85\%, 0.68 mmol (Lit. ${ }^{5}$ : $86 \%)]$ as a pale brown oil.

$\mathbf{R}_{\mathbf{f}}\left(\mathrm{PE} 30 / 50: \mathrm{Et}_{2} \mathrm{O}=3: 1\right)=0.22$

${ }^{1} \mathbf{H}$ NMR (300.13 MHz, $\left.\mathrm{CDCl}_{3} / \mathrm{CHCl}_{3}, \mathrm{DsBrNo} 21-30560,21.11 .2019\right): \delta=3.24$ (s, 3H, $N$ $\left.\mathrm{CH}_{3}\right), 3.72\left(\mathrm{~s}, 3 \mathrm{H}, O-\mathrm{CH}_{3}\right), 7.09\left(\mathrm{~d}, J_{2,3}=13.7 \mathrm{~Hz}, 1 \mathrm{H}, 2-\mathrm{H}\right), 7.58\left(\mathrm{~d}, J_{3,2}=13.7 \mathrm{~Hz}, 1 \mathrm{H}, 3-\mathrm{H}\right)$.

HRMS (ESI) m/z: [M+H] ${ }^{+}$Calcd for $\mathrm{C}_{5} \mathrm{H}_{8} \mathrm{BrNO}_{2}$ 193.9811; Found 193.9814

IR (KBr): $\tilde{v}=3000,2950,2840,1735,1615,1590,1510,1465,1440,1420,1365,1290,1265$, $1210,1175,1160,1130,1080,1035,935,920,835,790,765,750,710,640 \mathrm{~cm}^{-1}$.

\footnotetext{
${ }^{5}$ M. L. Hofferberth, R. Brückner, Angew. Chem. 2014, 126, 7456-7462; Angew. Chem. Int. Ed. 2014, 53, 7328 7334.
} 


\section{$t$-Butylthioacetate (24)}

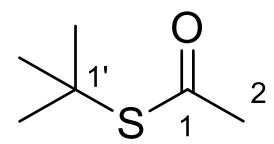

\section{4}

Pyridine $(2.89 \mathrm{~g}, 36.6 \mathrm{mmol}, 1.1 \mathrm{eq}$.$) was dissolved in chloroform (13.3 \mathrm{~mL})$ at $0^{\circ} \mathrm{C}$. It was added $\mathrm{AcCl}\left(2.80 \mathrm{~g}, 36.6 \mathrm{mmol}, 1.1 \mathrm{eq}\right.$.). The mixture stirred for $30 \mathrm{~min}$ at $0^{\circ} \mathrm{C} . t \mathrm{BuSH}(3.00 \mathrm{~g}$, $33.3 \mathrm{mmol}$ ) was added over a period of $3 \mathrm{~h}$. The mixture was warmed to $25^{\circ} \mathrm{C}$ and stirred at this temperature for $12 \mathrm{~h}$. The mixture was diluted with $\mathrm{H}_{2} \mathrm{O}(15 \mathrm{~mL})$. The organic layer was separated and washed with $\mathrm{H}_{2} \mathrm{O}(10 \mathrm{~mL})$, aq. $\mathrm{H}_{2} \mathrm{SO}_{4}(10$ wt.- $\%, 10 \mathrm{~mL})$, sat. aq. $\mathrm{NaHCO}_{3}$ solution $(10 \mathrm{~mL})$ and again with $\mathrm{H}_{2} \mathrm{O}(10 \mathrm{~mL})$. The organic layer was tried over $\mathrm{K}_{2} \mathrm{CO}_{3}$ und the solvent was removed under reduced pressure. The residue (bp. $\left.1013 \mathrm{mbar}: 130^{\circ} \mathrm{C}\right)$ was distillated to get the product 24 [3.17 g, 72\%, $24.0 \mathrm{mmol}$ (Lit. ${ }^{6}: 81 \%$ )] as a pale yellow liquid.

${ }^{1} \mathbf{H}$ NMR $\left(300.13 \mathrm{MHz}, \mathrm{CDCl}_{3} / \mathrm{CHCl}_{3}\right.$, DsBrMz23-30370, 23.3.2015): $\delta=1.46$ [s, 9H, 1'$\left.\left(\mathrm{CH}_{3}\right)_{3}\right], 2.23\left(\mathrm{~s}, 3 \mathrm{H}, 2-\mathrm{H}_{3}\right)$.

\section{S-tert-Butyl (E,E)-5-Bromo-3-hydroxypenta-2,4-dienethioate [enol-(11)]}

[pure (when crystallized)

or in an 85:15 mixture with the tautomeric]

S-tert-Butyl (E)-5-Bromo-3-oxopent-4-enethioate [keto-(11)]

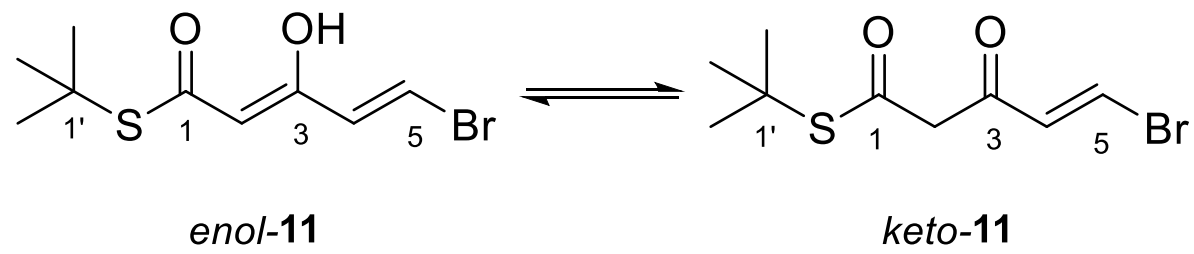

A solution of LiHMDS ( $1 \mathrm{M}$ in hexane, $0.77 \mathrm{~mL}, 0.77 \mathrm{mmol}, 1.5$ eq.) in THF (1.2 $\mathrm{mL})$ at $78^{\circ} \mathrm{C}$ was diluted with $t$ BuSAc (24) $(99 \mathrm{mg}, 0.78 \mathrm{mmol}, 1.6$ eq.) in THF (0.9 mL). After $1 \mathrm{~h}$ $\mathrm{MgBr}_{2} \cdot \mathrm{OEt}_{2}(290 \mathrm{mg}, 1.12 \mathrm{mmol}, 2.2 \mathrm{eq}$.) was added in one portion. After $1 \mathrm{~h}$ a solution of Weinreb amide $23(98 \mathrm{mg}, 0.51 \mathrm{mmol})$ in THF $(0.9 \mathrm{~mL})$ was added tropwise. After $2 \mathrm{~h}$ the

\footnotetext{
${ }^{6}$ P. N. Rylander, D. S. Tarbe, J. Am. Chem. Soc., 1950, 72, 3021-3025.
} 
solution was diluted with $\mathrm{HOAc}(0.5 \mathrm{~mL})$ and sat. aq. $\mathrm{NH}_{4} \mathrm{Cl}$ solution $(3 \mathrm{~mL})$. The mixture was warmed to room temperature and extracted with $\mathrm{CH}_{2} \mathrm{Cl}_{2}(3 \times 10 \mathrm{~mL})$. The combined organic layers were dried over $\mathrm{Na}_{2} \mathrm{SO}_{4}$ and concentrated under reduced pressure. Purification by flash chromatography $\left(c \mathrm{C}_{6} \mathrm{H}_{12}: \mathrm{AcOEt}=75: 1,1.5 \mathrm{~cm}, 15 \mathrm{~cm}, 8 \mathrm{~mL}\right)$ afforded 11 [101 mg, 75\%, 0.38 mmol (Lit. $\left.{ }^{5}: 83 \%\right)$ ] as a red oil, which became solid (mp.: $51^{\circ} \mathrm{C}$ ) after $12 \mathrm{~h}$ at $-20^{\circ} \mathrm{C}$.

$\mathbf{R}_{\mathbf{f}}\left(c \mathrm{C}_{6} \mathrm{H}_{12}: \mathrm{AcOEt}=4: 1\right)=0.90$

${ }^{1} \mathbf{H}$ NMR $\left(300.13 \mathrm{MHz}, \mathrm{CDCl}_{3} / \mathrm{CHCl}_{3}\right.$, sample contained unknown impurity with a s at 1.46, DsBrNo26-30370, 26.11.2019): $\delta=1.48$ [s, 9H, 1'-( $\left.\mathrm{CH}_{3}\right)_{3}$ (keto-11)], 1.52 [s, 9H, 1'-( $\left.\mathrm{CH}_{3}\right)_{3}$ (enol-11)], 3.68 [s, 2H, 2- $\mathrm{H}_{2}$ (keto-11)], 5.32 [s, 1H, 2-H (enol-11)], 6.36 [dd, $J_{4,5}=13.6 \mathrm{~Hz}$, ${ }^{4} J_{4,3-\mathrm{OH}}=1.6 \mathrm{~Hz}, 1 \mathrm{H}, 4-\mathrm{H}($ enol-11) $], 6.90\left[\mathrm{~d}, J_{4,5}=14.0 \mathrm{~Hz}, 1 \mathrm{H}, 4-\mathrm{H}(\right.$ keto-11), 7.27 [d, $J_{5,4}=13.6 \mathrm{~Hz}, 1 \mathrm{H}, 5-\mathrm{H}\left(\right.$ enol-11), $7.66\left[\mathrm{~d}, J_{5,4}=14.0 \mathrm{~Hz}, 1 \mathrm{H}, 5-\mathrm{H},\left(\right.\right.$ keto-11), $12.44\left[\mathrm{~d},{ }^{4} J_{3-}\right.$ $\mathrm{OH}, 4=1.7 \mathrm{~Hz}, 1 \mathrm{H}, 3-\mathrm{OH}($ enol-11) $]$.

HRMS (ESI) m/z: [M-H] ${ }^{+}$Calcd for $\mathrm{C}_{9} \mathrm{H}_{12} \mathrm{O}_{2} \mathrm{BrS} 262.9747$; Found 262.9746

IR (KBr): $\tilde{v}=3085,2965,2925,2865,1625,1575,1495,1475,1455,1400,1365,1325,1285$, $1205,1170,1075,1025,985,930,860,820,760,700,685,610,530,510 \mathrm{~cm}^{-1}$.

Methyl (2S)-Amino-3-(4'-hydroxyphenyl)propanoate [(S)-20]

and

\section{Methyl (2R)-Amino-3-(4'-hydroxyphenyl)propanoate [(R)-20]}<smiles>COC(=O)[C@H](N)Cc1ccc(O)cc1</smiles>

$(S)-20$<smiles>COC(=O)[C@H](N)Cc1ccc(O)cc1</smiles>

$(R)-20$

Thionyl chloride ( $4.00 \mathrm{~mL}, 6.55 \mathrm{~g}, 55.0 \mathrm{mmol}, 1.8 \mathrm{eq}$.) was added dropwise to a suspension of $L$-tyrosine $(\mathbf{1 6})(5.45 \mathrm{~g}, 30.1 \mathrm{mmol})$ in $\mathrm{MeOH}(100 \mathrm{~mL})$ at $0^{\circ} \mathrm{C}$. The mixture was allowed to 
Christian Drescher, Morris Keller, Olivier Potterat, Matthias Hamburger and Reinhard Brückner, Structure-Elucidating Total Synthesis of the (Polyenoyl)tetramic Acid Militarinone C

warm to $25^{\circ} \mathrm{C}$ and stirred overnight. The solvent was removed under reduced pressure and the residue was washed with $\mathrm{Et}_{2} \mathrm{O}(50 \mathrm{~mL})$. The product $(S)-20$ [5.58 g, 95\%, 28.6 mmol (Lit. : 99\%)] was obtained as a white solid.

The enantiomer $(R)$-20 was obtained analogously in $95 \%$ yield.

${ }^{1} \mathrm{H}$ NMR (300.13 MHz, $\left.\mathrm{D}_{2} \mathrm{O} / \mathrm{H}_{2} \mathrm{O}, \mathrm{DsBrAp} 15-30500,15.4 .2015\right): \delta=\mathrm{AB}$ signal $\left(\delta_{\mathrm{A}}=3.21\right.$, $\delta_{\mathrm{B}}=3.30, J_{\mathrm{AB}}=14.6 \mathrm{~Hz}$, in addition split by $\left.J_{3-\mathrm{H}^{\mathrm{A}}, 2}=5.8 \mathrm{~Hz}, J_{3-\mathrm{H}^{\mathrm{B}}, 2}=7.5 \mathrm{~Hz}, 2 \mathrm{H}, 3-\mathrm{H}_{2}\right), 3.87$ $\left(\mathrm{s}, 3 \mathrm{H}, 1-\mathrm{OCH}_{3}\right), 4.41\left(\mathrm{dd}, J_{2,3-\mathrm{H}^{\mathrm{A}}}=6.0 \mathrm{~Hz}, J_{2,3-\mathrm{H}^{\mathrm{B}}}=7.4 \mathrm{~Hz}, 1 \mathrm{H}, 2-\mathrm{H}\right), 6.93\left(\mathrm{~m}, J_{3^{\prime}, 5^{\prime} ; 2^{\prime}, 6^{\prime}}=8.7 \mathrm{~Hz}\right.$, 2H, 3'-H, 5'-H), 7.20 (d, J $\left.J_{2}^{\prime}, 6^{\prime} 3^{\prime}, 5^{\prime}=8.7 \mathrm{~Hz}, 2 \mathrm{H}, 2^{\prime}-\mathrm{H}, 66^{\prime}-\mathrm{H}\right)$.

Specific rotation $(S)-20[a]_{D}^{20}=+13.1^{\circ}\left(\mathrm{c}=1.0\right.$ in $\left.\mathrm{H}_{2} \mathrm{O}\right)$.

Specific rotation $(R)-20[a]_{D}^{20}=-12.2^{\circ}\left(\mathrm{c}=1.0\right.$ in $\left.\mathrm{H}_{2} \mathrm{O}\right)$.

Methyl (2S)-2-Amino-3-\{4'-[(tert-butyldimethylsilyl)oxy]phenyl\}propanoate $[(S)-21]$ and

\section{Methyl (2R)-2-Amino-3-\{4'-[(tert-butyldimethylsilyl)oxy]phenyl\}propanoate [(R)-21]}

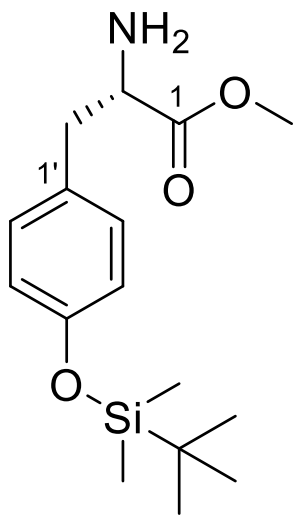

$(S)-21$<smiles>COC(=O)[C@H](N)Cc1ccc(O[Si](C)(C)C(C)(C)C)cc1</smiles>

$(R)-21$

A suspension of $L$-tyrosine methyl ester (20) $(500 \mathrm{mg}, 2.55 \mathrm{mmol})$ in $\mathrm{CH}_{2} \mathrm{Cl}_{2}(25 \mathrm{~mL})$ was treated with imidazole (438 mg, $6.34 \mathrm{mmol}, 2.5$ eq.) and $t \mathrm{BuMe}{ }_{2} \mathrm{SiCl}$ (461 mg, $3.06 \mathrm{mmol}, 1.2$ eq.). The mixture stirred at $25^{\circ} \mathrm{C}$ for $14 \mathrm{~h}$ before being quenched with sat. aq. $\mathrm{NH}_{4} \mathrm{Cl}$ solution $(25 \mathrm{~mL})$. The layers were separated and aq. layer was extracted with $\mathrm{CH}_{2} \mathrm{Cl}_{2}(3 \times 15 \mathrm{~mL})$. The combined organic layers were dried over $\mathrm{MgSO}_{4}$ and concentrated under reduced pressure.

${ }^{7}$ L. Raffier, O. Piva, Beilstein J. Org. Chem. 2011, 7, 151-155. 
Purification by flash chromatography (AcOEt, $2.5 \mathrm{~cm}, 15 \mathrm{~cm}, 20 \mathrm{~mL}$ ) afforded $(S)-27[667 \mathrm{mg}$, 84\%, 2.14 mmol (Lit. ${ }^{8}: 86 \%$ )] as a colorless oil.

The enantiomer $(R)$-21 was obtained analogously in $85 \%$ yield.

$\mathbf{R}_{\mathbf{f}}(\mathrm{AcOEt})=0.45$

${ }^{1} \mathbf{H}$ NMR $\left(300.13 \mathrm{MHz}, \mathrm{CDCl}_{3} / \mathrm{CHCl}_{3}\right.$, DsBrNo21-30550, 21.11.2019): $\delta=0.18$ [s, 6H, Si$\left(\mathrm{CH}_{3}\right)_{2}$ ], 0.97 [s, 9H, Si-C(CH$)_{3}$ ], 1.60 (br. s, $\left.2 \mathrm{H}, 2-\mathrm{NH}_{2}\right), \mathrm{AB}$ signal $\left(\delta_{\mathrm{A}}=2.81, \delta_{\mathrm{B}}=3.00\right.$, $J_{\mathrm{AB}}=13.6 \mathrm{~Hz}$, in addition split by $\left.J_{3-\mathrm{H}^{\mathrm{A}}, 2}=5.4 \mathrm{~Hz}, J_{3-\mathrm{H}^{\mathrm{B}}, 2}=7.8 \mathrm{~Hz}, 2 \mathrm{H}, 3-\mathrm{H}_{2}\right), 3.69\left(\mathrm{dd}, J_{2,3-\mathrm{H}^{\mathrm{B}}}\right.$ $\left.=5.3 \mathrm{~Hz}, J_{2,3-\mathrm{H}^{\mathrm{A}}}=7.8 \mathrm{~Hz}, 1 \mathrm{H}, 2-\mathrm{H}\right), 3.70\left(\mathrm{~s}, 3 \mathrm{H}, 1-\mathrm{OCH}_{3}\right), 6.77\left(\mathrm{~d}, J_{3^{\prime}, 5^{\prime} ; 2^{\prime}, 6^{\prime}}=8.7 \mathrm{~Hz}, 2 \mathrm{H}, 3^{\prime}-\mathrm{H}\right.$, 5'-H), $7.03\left(\mathrm{~d}, J_{2^{\prime}, 6^{\prime} ; 3^{\prime}, 5^{\prime}}=8.6 \mathrm{~Hz}, 2 \mathrm{H}, 2^{\prime}-\mathrm{H}, 6^{\prime}-\mathrm{H}\right)$.

Specific rotation $(S)-21[a]_{D}^{20}=+4.9^{\circ}\left(\mathrm{c}=1.0\right.$ in $\left.\mathrm{CHCl}_{3}\right)$.

Specific rotation $(R)-21[a]_{D}^{20}=-5.1^{\circ}\left(\mathrm{c}=1.0\right.$ in $\left.\mathrm{CHCl}_{3}\right)$.

Methyl (2S)-3-\{4'-[(tert-Butyldimethylsilyl)oxy]phenyl\}-2-[(1",5'"dimethoxybenzyl)amino]propanoate $[(S)-13]$

and

Methyl (2R)-3-\{4'-[(tert-Butyldimethylsilyl)oxy]phenyl $\}-2-\left[\left(1^{\prime \prime}, 5^{\prime \prime}-\right.\right.$ dimethoxybenzyl)amino]propanoate $[(S)-13]$<smiles>COC(=O)[C@H](Cc1ccc(O[Si](C)(C)C(C)(C)C)cc1)NCC1=[C+]C=C(OC)C=C1OC</smiles>

(S)-13<smiles>COC(=O)[C@H](Cc1ccc(O[Si](C)(C)C(C)(C)C)cc1)NCc1ccc(OC)cc1OC</smiles>

$(R)-13$

\footnotetext{
${ }^{8}$ N. Riache, C. Bailly, A. Deville, L. Dubost, B. Nay, Eur. J. Org. Chem. 2010, 5402-5408.
} 
The amine $(S)-21(250 \mathrm{mg}, 0.80 \mathrm{mmol})$ was dissolved in $\mathrm{MeOH}(7.5 \mathrm{~mL})$. It was added HOAc (5 drops), 2,4-dimethoxybenzaldehyde (135 mg, $0.88 \mathrm{mmol}, 1.1$ eq.) and immediately (not more than $10 \mathrm{sec}) \mathrm{NaBH}_{3} \mathrm{CN}$ (79 mg, $1.20 \mathrm{mmol}, 1.5 \mathrm{eq}$.). The mixture was stirred at $25^{\circ} \mathrm{C}$ for $2.25 \mathrm{~h}$. The solvent was removed under reduced pressure. It was added $\mathrm{H}_{2} \mathrm{O}(5 \mathrm{~mL})$ and $\mathrm{Et}_{2} \mathrm{O}$ $(10 \mathrm{~mL})$. The layers were separated and aq. layer was extracted with $\mathrm{Et}_{2} \mathrm{O}(3 \times 15 \mathrm{~mL})$. The combined organic layers were dried over $\mathrm{MgSO}_{4}$ and concentrated under reduced pressure. Purification by flash chromatography $\left(c \mathrm{C}_{6} \mathrm{H}_{12}: \mathrm{AcOEt}=2: 1,3.0 \mathrm{~cm}, 20 \mathrm{~cm}, 20 \mathrm{~mL}\right)$ afforded (S)-13 (260 mg, 70\%, $0.56 \mathrm{mmol}, 99.5 \%$ ee ) as a colorless oil.

The enantiomer $(R)$-13 was obtained analogously in $69 \%$ yield.

$\mathbf{R}_{\mathbf{f}}\left(c \mathrm{C}_{6} \mathrm{H}_{12}: \mathrm{AcOEt}=2: 1\right)=0.35$

${ }^{1} \mathbf{H}$ NMR $\left(500.10 \mathrm{MHz}, \mathrm{CDCl}_{3} / \mathrm{CHCl}_{3}, \mathrm{DsBrNo} 07-31160,28.11 .2019\right): \delta=0.18$ [s, 6H, Si$\left.\left(\mathrm{CH}_{3}\right)_{2}\right], 0.97$ [s, 9H, Si-C $\left.\left(\mathrm{CH}_{3}\right)_{3}\right], 1.97$ (br. s, $\left.1 \mathrm{H}, N \mathrm{H}\right), \mathrm{AB}$ signal $\left(\delta_{\mathrm{A}}=2.85, \delta_{\mathrm{B}}=2.89\right.$, $J_{\mathrm{AB}}=13.4 \mathrm{~Hz}$, in addition split by $\left.J_{3-\mathrm{H}^{\mathrm{A}}, 2}=6.6 \mathrm{~Hz}, J_{3-\mathrm{H}^{\mathrm{B}}, 2}=7.6 \mathrm{~Hz}, 2 \mathrm{H}, 3-\mathrm{H}_{2}\right), 3.45(\mathrm{t}$, $\left.J_{2,3}=6.7 \mathrm{~Hz}, 1 \mathrm{H}, 2-\mathrm{H}\right), 3.59\left(\mathrm{~s}, 3 \mathrm{H}, 1-\mathrm{OCH}_{3}\right), \mathrm{AB}$ signal $\left(\delta_{\mathrm{A}}=3.61, \delta_{\mathrm{B}}=3.72, J_{\mathrm{AB}}=13.4 \mathrm{~Hz}\right.$, $\left.2 \mathrm{H}, \mathrm{N}-\mathrm{CH}_{2}\right), 3.68$ (s, 3H, 1"--OCH 3$), 3.78$ (s, 3H, 5"--OCH $), 6.35-6.41$ (m, 2H, 4"-H, 6"-H), 6.67-6.76 (m, 2H, 3'-H, 5'-H), 6.94-7.05 (m, 1H, 3'-H), 6.94-7.05 (m, 2H, 2'-H, 6'-H).

${ }^{13} \mathrm{C}$ NMR (125.75 MHz, $\mathrm{CDCl}_{3} / \mathrm{CHCl}_{3}$, DsBrMi21-5076, 28.11.2019): $\delta=-4.3$ [Si-( $\left.\left(\mathrm{CH}_{3}\right)_{2}\right]$, $18.3\left[\mathrm{Si}-\mathrm{C}\left(\mathrm{CH}_{3}\right)_{3}\right], 25.8$ [Si-C $\left.\left(\mathrm{CH}_{3}\right)_{3}\right], 39.0(\mathrm{C}-3), 47.3\left(\mathrm{~N}-\mathrm{CH}_{2}\right), 51.6\left(1-\mathrm{OCH}_{3}\right), 55.2(1 "-$ $\mathrm{OCH}_{3}$ ), 55.4 (5"--OCH 3 ), 62.3 (C-2), 98.5 (C-4"), 103.7 (C-6"), 120.0 (C-3', C-5'), 120.2 (C-2"), 130.2 (C-1', C-2', C-6'), 130.4 (C-3"), 154.4 (C-4'), 158.7 (C-1"), 160.2 (C-5"), 175.1 (C-1).

edHSQC [,,short-range H,C-COSY“, $500.32 \mathrm{MHz} / 125.80 \mathrm{MHz}, \mathrm{CDCl}_{3} ; \delta\left({ }^{1} \mathrm{H}\right) \leftrightarrow \delta\left({ }^{13} \mathrm{C}\right): 0.18$ $\left[\mathrm{s}, 6 \mathrm{H}, \mathrm{Si}-\left(\mathrm{CH}_{3}\right)_{2}\right] \leftrightarrow-4.3\left[\mathrm{Si}-\left(\mathrm{CH}_{3}\right)_{2}\right], 0.98\left[\mathrm{~s}, \mathrm{Si}-\mathrm{C}\left(\mathrm{CH}_{3}\right)_{3}\right] \leftrightarrow 25.8\left[\mathrm{Si}-\mathrm{C}\left(\mathrm{CH}_{3}\right)_{3}\right], \mathrm{AB}$ signal $\left(\delta_{\mathrm{A}}=2.85, \delta_{\mathrm{B}}=2.89,3-\mathrm{H}_{2}\right) \leftrightarrow 39.0(\mathrm{C}-3), 3.45(\mathrm{t}, 2-\mathrm{H}) \leftrightarrow 62.3(\mathrm{C}-2), 3.59\left(\mathrm{~s}, 3 \mathrm{H}, 1-\mathrm{OCH}_{3}\right) \leftrightarrow$ $51.6\left(1-\mathrm{OCH}_{3}\right), \mathrm{AB}$ signal $\left(\delta_{\mathrm{A}}=3.61, \delta_{\mathrm{B}}=3.72, N-\mathrm{CH}_{2}\right) \leftrightarrow 47.3\left(N-\mathrm{CH}_{2}\right), 3.68\left(\mathrm{~s}, 1 "-\mathrm{OCH}_{3}\right)$ $\leftrightarrow 55.2\left(1 "-\mathrm{OCH}_{3}\right), 3.78\left(\mathrm{~s}, 5 "-\mathrm{OCH}_{3}\right) \leftrightarrow 55.4\left(5 "-\mathrm{OCH}_{3}\right), 6.35-6.41(\mathrm{~m}, 4 "-\mathrm{H}) \leftrightarrow 98.5(\mathrm{C}-4 ")$, 6.35-6.41 (m, 6"-H) 103.7 (C-6"), 6.67-6.76 (m, 3'-H, 5'-H) 120.0 (C-3', C-5'), 6.94-7.05 $\left(\mathrm{m}, 3^{\prime \prime}-\mathrm{H}\right) \leftrightarrow 130.4$ (C-3"), 6.94-7.05 (m, 2'-H, 6'-H) 130.2 (C-2', C-6').

Specific rotation $(S)-\mathbf{1 3}[a]_{D}^{20}=+14.9^{\circ}\left(\mathrm{c}=1.0\right.$ in $\left.\mathrm{CHCl}_{3}\right)$.

Specific rotation $(R)-13[a]_{D}^{20}=-15.5^{\circ}\left(\mathrm{c}=1.0\right.$ in $\left.\mathrm{CHCl}_{3}\right)$.

HRMS (ESI) m/z: [M+H] $]^{+}$Calcd for $\mathrm{C}_{25} \mathrm{H}_{38} \mathrm{O}_{5} \mathrm{NSi} 460.2514$; Found 460.2517 
IR (KBr): $\tilde{v}=3000,2955,2930,2860,1730,1610,1590,1510,1465,1440,1290,1260,1210$, $1155,1130,1040,915,840,780,690 \mathrm{~cm}^{-1}$.

Methyl (2'S)-2'-\{N-[(1E,2Z,4E)-5-Bromo-3-hydroxy-1-oxoypenta-2,4-dienyl]- $N$-(1"',5"'dimethoxybenzyl) $\}-3 '-\left\{1^{\prime \prime}-[(\right.$ tert-butyldimethylsilyl)oxy $]$ phenyl $\}$ propanoate $[$ E-enol-(S)-

8]

in an equilibrium with

Methyl (2'S)-2'-\{N-[(1Z,2Z,4E)-5-Bromo-3-hydroxy-1-oxoypenta-2,4-dienyl]- $N$-(1'",5'"'dimethoxybenzyl) $\}-3$ '- $\left\{1^{\prime \prime}-[(\right.$ tert-butyldimethylsilyl $)$ oxy $]$ phenyl $\}$ propanoate $[Z$-enol-(S)8]

in an equilibrium with

Methyl (2'S)-2'- $\left\{N\right.$-[(1E,4E)-5-Bromo-1,3-dioxopent-4-enyl]- $N-\left(1 '{ }^{\prime \prime}, 5\right.$ '"dimethoxybenzyl $)\}-3$ '-\{1"-[(tert-butyldimethylsilyl)oxy $]$ phenyl $\}$ propanoate $[$ E-keto-(S)8] in an equilibrium with

Methyl (2'S)-2'-\{ $N$-[(1Z,4E)-5-Bromo-1,3-dioxopent-4-enyl]- $N-\left(1{ }^{\prime \prime}, 5\right.$ '"'dimethoxybenzyl $)\}-3$ '- $\left\{1^{\prime \prime}-[(\right.$ tert-butyldimethylsilyl)oxy]phenyl $\}$ propanoate $[$ Z-keto-(S)8] and

Methyl (2'R)-2' - $\{N$-[(1E,2Z,4E)-5-Bromo-3-hydroxy-1-oxoypenta-2,4-dienyl]- $N$-(1"',5'"'dimethoxybenzyl $)\}-3$ '- $\left\{1^{\prime \prime}-[(\right.$ tert-butyldimethylsilyl)oxy $]$ phenyl $\}$ propanoate $[E-e n o l-(R)-$ 8]

in an equilibrium with

Methyl (2'R)-2'-\{N-[(1Z,2Z,4E)-5-Bromo-3-hydroxy-1-oxoypenta-2,4-dienyl]- $N$-(1'"',5"'dimethoxybenzyl) $\}-3^{\prime}-\left\{1^{\prime \prime}-[(\right.$ tert-butyldimethylsilyl $)$ oxy $]$ phenyl $\}$ propanoate $[Z$-enol-(R)8] 
Christian Drescher, Morris Keller, Olivier Potterat, Matthias Hamburger and Reinhard Brückner, Structure-Elucidating Total Synthesis of the (Polyenoyl)tetramic Acid Militarinone C

Methyl (2'R)-2'-\{N-[(1E,4E)-5-Bromo-1,3-dioxopent-4-enyl]- $N-(1 ' ", 5 ' " '-$

dimethoxybenzyl $)\}-3 '-\left\{1^{\prime \prime}-[(\right.$ tert-butyldimethylsilyl $)$ oxy $]$ phenyl $\}$ propanoate $[$ E-keto- $(R)-$ 8]

in an equilibrium with

Methyl (2'R)-2'-\{N-[(1Z,4E)-5-Bromo-1,3-dioxopent-4-enyl]- $N-\left(1{ }^{\prime \prime}, 5\right.$ '"'-

dimethoxybenzyl) $\}-3$ '-\{1'-[(tert-butyldimethylsilyl)oxy]phenyl $\}$ propanoate $[$ Z-keto-(R)-

8] 
Christian Drescher, Morris Keller, Olivier Potterat, Matthias Hamburger and Reinhard Brückner, Structure-Elucidating Total Synthesis of the (Polyenoyl)tetramic Acid Militarinone C<smiles>CC=CC(O)=CC(=O)N(Cc1ccc(OC)c[14c]1OC)[C@@H](Cc1ccc(O[Si](C)(C)C(C)(C)C)cc1)C(=O)OC</smiles>

E-enol-(S)-8<smiles>[CH]=C</smiles><smiles></smiles>

E-keto-(S)-8<smiles>CO[Te]c1cc(OC)ccc1CN(C(=O)/C=C(O)/C=C/Br)[C@@H](Cc1ccc(O[Si](C)(C)C(C)(C)C)cc1)C(=O)OC</smiles>

Z-enol-(S)-8<smiles>[CH-]=C</smiles><smiles>COC(=O)[C@H](Cc1ccc(O[Si](C)(C)C(C)(C)C)cc1)N(Cc1ccc(OC)cc1OC)C(=O)CC(=O)/C=C/Br</smiles>

Z-keto-(S)-8

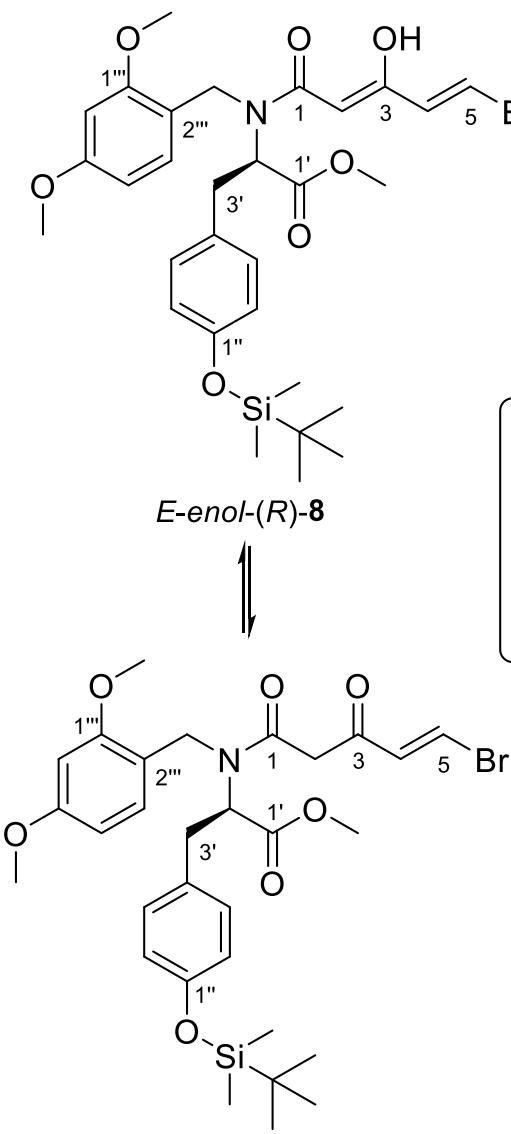


A solution of $N$-DMB-tyrosine methyl ester $(S)-13(73.0 \mathrm{mg}, 220 \mu \mathrm{mol})$ and the $\beta$-ketothioester 11 (66.0 mg, $330 \mu \mathrm{mol}, 1.5 \mathrm{eq}$.) in THF (1.9 mL) was treated with powdered molecular sieves $4 \AA(0.4 \mathrm{~g})$. The suspension stirred at $25^{\circ} \mathrm{C}$ for $30 \mathrm{~min}$. Then $\mathrm{AgO}_{2} \mathrm{CCF}_{3}(65.1 \mathrm{mg}, 330 \mu \mathrm{mol}$, 1.5 eq.) was added and the mixture was stirred for $20 \mathrm{~h}$ at $25^{\circ} \mathrm{C}$. The solvent was remove under reduced pressure and $\mathrm{Et}_{2} \mathrm{O}(10 \mathrm{~mL})$ was added to the resulting suspension. The mixture was filtered and the filtrate was concentrated under reduced pressure. Purification by flash chromatography $\left(c \mathrm{C}_{6} \mathrm{H}_{12}:\right.$ AcOEt $\left.=5: 1,2.0 \mathrm{~cm}, 20 \mathrm{~cm}, 15 \mathrm{~mL}\right)$ afforded $(S)-8(65.2 \mathrm{mg}, 65 \%$, $141 \mu \mathrm{mol}, 99 \% \mathrm{ee}$ ) as a red oil. It represented a mixture of two rotamers in a keto and an enol form each [E-enol-(S)-8:Z-enol-(S)-8:E-keto-(S)-8:Z-keto-(S)-8=84:12:2:1]. This ratio was determined by integrating the 5-H signals [at $\delta=7.18$ (E-enol), 7.53 (Z-keto), 7.60 (E-keto) and $7.68(Z$-enol $) \mathrm{ppm}]$ and compared with the 3-OH signals [14.00 (E-enol) and $14.08(Z$-enol $)]$.

The enantiomer $(R)-8$ was obtained analogously in $66 \%$ yield.

$\mathbf{R}_{\mathbf{f}}\left(c \mathrm{C}_{6} \mathrm{H}_{12}: \mathrm{AcOEt}=5: 1\right)=0.38$

\section{E-enol-(S)-8:}

${ }^{1} \mathbf{H}$ NMR $\left(500.10 \mathrm{MHz}, \mathrm{CDCl}_{3} / \mathrm{CHCl}_{3}\right.$, sample contained grease, $\mathrm{H}_{2} \mathrm{O}$, trace amounts of AcOEt and unknown impurity $\mathrm{m}$ at 5.05 and dd at 6.08, DsBrMi21-5070, 26.8.2015): $\delta=0.18[\mathrm{~s}, 6 \mathrm{H}$, $\left.\mathrm{Si}-\left(\mathrm{CH}_{3}\right)_{2}\right], 0.98\left[\mathrm{~s}, 9 \mathrm{H}, \mathrm{Si}-\mathrm{C}\left(\mathrm{CH}_{3}\right)_{3}\right], \mathrm{AB} \operatorname{signal}\left(\delta_{\mathrm{A}}=3.12, \delta_{\mathrm{B}}=3.29, J_{\mathrm{AB}}=14.1 \mathrm{~Hz}\right.$, in addition split by $\left.J_{3^{\prime}-\mathrm{H}^{\mathrm{A}}, 2^{\prime}}=6.0 \mathrm{~Hz}, J_{3^{\prime}-\mathrm{H}^{\mathrm{B}}, 2^{\prime}}=9.0 \mathrm{~Hz}, 2 \mathrm{H}, 3^{\prime}-\mathrm{H}_{2}\right), 3.61$ (s, 3H, 1'-OCH $), 3.75$ (s, 3H, 1'"$\left.\mathrm{OCH}_{3}\right), 3.79$ (s, 3H, 5'"-OCH $), 3.90\left(\mathrm{~d},{ }^{2} J=17.2 \mathrm{~Hz}, 1 \mathrm{H}, 2\right.$ ''-C-H $\left.{ }^{\mathrm{A}}\right), 4.32\left(\mathrm{~d},{ }^{2} J=17.2 \mathrm{~Hz}, 1 \mathrm{H}\right.$,

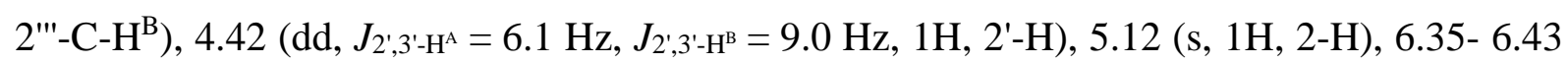
(m, 3H, 4"'-H, 6"'-H, 4-H), 6.70-6.74 (m, 2H, 2"-H, 6"-H), 6.93-6.98 (m, 2H, 3"-H, 5"-H), 6.93$6.98\left(\mathrm{~m}, 1 \mathrm{H}, 3^{\prime \prime}-\mathrm{H}\right), 7.18\left(\mathrm{~d}, J_{5,4}=13.6 \mathrm{~Hz}, 1 \mathrm{H}, 5-\mathrm{H}\right), 14.00\left(\mathrm{~d},{ }^{4} J_{3-\mathrm{OH}, 2}=1.5 \mathrm{~Hz}, 1 \mathrm{H}, 3-\mathrm{OH}\right)$.

${ }^{13} \mathrm{C}$ NMR (125.75 MHz, $\left.\mathrm{CDCl}_{3} / \mathrm{CHCl}_{3}, \mathrm{DsBrMi} 21-5076,26.8 .2015\right): \delta=-4.3\left[\mathrm{Si}-\left(\mathrm{CH}_{3}\right)_{2}\right]$, $18.3\left[\mathrm{Si}-\mathrm{C}\left(\mathrm{CH}_{3}\right)_{3}\right], 25.8$ [Si-C $\left.\left(\mathrm{CH}_{3}\right)_{3}\right], 34.7\left(\mathrm{C}-3\right.$ '), $47.1\left(2\right.$ "'- $\left.\mathrm{CH}_{2}\right), 52.3\left(1\right.$ '- $\left.-\mathrm{OCH}_{3}\right), 55.2(1$ '"$\mathrm{OCH}_{3}$ ), 55.5 (5'"-OCH 3$), 60.9$ (C-2'), 90.7 (C-2), 98.3 (C-4'"), 103.8 (C-6'"), 116.3 (C-5), 116.6 (C-2"'), 120.2 (C-2", C-6"), 129.3 (C-3"'), 130.3 (C-3", C-5"), 130.5 (C-4"), 132.8 (C-4), 154.4 (C-1"), 157.9 (C-1"'), 160.7 (C-5"'), 166.9 (C-3), 171.1 (C-1'), 172.6 (C-1).

edHSQC [,,short-range H,C-COSY“،, $500.32 \mathrm{MHz} / 125.80 \mathrm{MHz}, \mathrm{CDCl}_{3} ; \delta\left({ }^{1} \mathrm{H}\right) \leftrightarrow \delta\left({ }^{13} \mathrm{C}\right): 0.18$ $\left[\mathrm{s}, \mathrm{Si}-\left(\mathrm{CH}_{3}\right)_{2}\right] \leftrightarrow-4.3\left[\mathrm{Si}-\left(\mathrm{CH}_{3}\right)_{2}\right], 0.98\left[\mathrm{~s}, \mathrm{Si}-\mathrm{C}\left(\mathrm{CH}_{3}\right)_{3}\right] \leftrightarrow 25.8\left[\mathrm{Si}-\mathrm{C}\left(\mathrm{CH}_{3}\right)_{3}\right], \mathrm{AB}$ signal $\left(\delta_{\mathrm{A}}\right.$ $\left.=3.12, \delta_{\mathrm{B}}=3.29,3^{\prime}-\mathrm{H}_{2}\right) \leftrightarrow 34.7\left(\mathrm{C}-3\right.$ '), $3.61\left(\mathrm{~s}, 1^{\prime}-\mathrm{OCH}_{3}\right) \leftrightarrow 52.2\left(1^{\prime}-\mathrm{OCH}_{3}\right), 3.75\left(\mathrm{~s}, 1^{\prime \prime}-\mathrm{OCH}_{3}\right)$

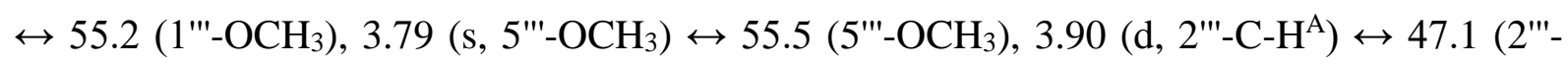


Christian Drescher, Morris Keller, Olivier Potterat, Matthias Hamburger and Reinhard Brückner, Structure-Elucidating Total Synthesis of the (Polyenoyl)tetramic Acid Militarinone C

$\left.\mathrm{CH}_{2}\right), 4.32\left(\mathrm{~d}, 2^{\prime \prime}-\mathrm{C}-\mathrm{H}^{\mathrm{B}}\right) \leftrightarrow 47.1\left(2^{\prime \prime}-\mathrm{CH}_{2}\right), 4.42\left(\mathrm{dd}, 2^{\prime}-\mathrm{H}\right) \leftrightarrow 60.9(\mathrm{C}-2 '), 5.12(\mathrm{~s}, 2-\mathrm{H}) \leftrightarrow 90.7$ (C-2), 6.35- 6.43 (m, 4'"-H) ↔ 98.3 (C-4'"), 6.43 (m, 6"'-H) 103.8 (C-6"'), 6.41 (m, 4-H) ↔ 132.8 (C-4), 6.70-6.74 (m, 2"-H, 6"-H) ↔ 120.2 (C-2", C-6"), 6.93-6.98 (m, 3"-H, 5"-H) ↔ 130.3 (C-3", C-5"), 6.93-6.98 (m, 3"'-H) ↔ 129.3 (C-3"'), 7.18 (d, 5-H) 116.3 (C-5).

Specific rotation $(S)-8[a]_{D}^{20}=-189.3^{\circ}\left(\mathrm{c}=1.0\right.$ in $\left.\mathrm{CHCl}_{3}\right)$.

Specific rotation $(R)-8[a]_{D}^{20}=+199.5^{\circ}\left(\mathrm{c}=1.0\right.$ in $\left.\mathrm{CHCl}_{3}\right)$.

HRMS (ESI) m/z: [M+H] $]^{+}$Calcd for $\mathrm{C}_{30} \mathrm{H}_{41} \mathrm{O}_{7} \mathrm{NBrSi}$ 634.1830; Found 634.1830

IR $(\mathrm{KBr}): \tilde{v}=2955,2930,2855,1745,1635,1585,1470,1390,1290,1260,1210,1170,1160$, 1120, 1040, 915, 840, 780, $695 \mathrm{~cm}^{-1}$. 


\subsection{Synthesis of the two Enantiomers of the Eastern Block Precursor 32}

$N$-[(1Z,1'S,2'S)-2'-Hydroxy-2'-phenylpropan-1'-yl]- $N$-methylpropionamide $[(Z, S, S)-18]$ in an 67:33 mixture with

$N-\left[\left(1 E, 1^{\prime} S, 2 ' S\right)-2\right.$ '-Hydroxy-2'-phenylpropan-1'-yl]- $N$-methylpropionamide $[(E, S, S)-18]$ and

$N$-[(1Z,1'R,2'R)-2'-Hydroxy-2'-phenylpropan-1'-yl]- $N$-methylpropionamide $[(Z, R, R)-18]$ in an 67:33 mixture with

$N$-[(1E,1'R,2'R)-2'-Hydroxy-2'-phenylpropan-1'-yl]- $N$-methylpropionamide $[(E, R, R)-18]$<smiles>CCC(=O)N(C)[C@@H](C)[C@H](O)c1ccccc1</smiles><smiles>CCC(=O)N(C)[C@@H](C)[C@H](O)c1ccccc1</smiles>

$(Z, S, S)-18$

$(E, S, S)-\mathbf{1 8}$<smiles>CCC(=O)N(C)[C@@H](C)[C@@H](O)c1ccccc1</smiles>

$(Z, R, R)-18$<smiles>CCC(=O)N(C)[C](C)[C@H](O)c1ccccc1</smiles>

$(E, R, R)-18$

A solution of pseudoephedrine $[(S, S)-25](2.10 \mathrm{~g}, 12.7 \mathrm{mmol})$ and $\mathrm{NEt}_{3}(1.55 \mathrm{~g}, 15.3 \mathrm{mmol}$, 1.2 eq.) in $\mathrm{CH}_{2} \mathrm{Cl}_{2}(25 \mathrm{~mL})$ was treated with propionic anhydride (1.72 g, $13.6 \mathrm{mmol}, 1.1$ eq.). After $30 \mathrm{~min}$ at $25^{\circ} \mathrm{C}$ the mixture was diluted with $\mathrm{H}_{2} \mathrm{O}(10 \mathrm{~mL})$. The organic layer was washed with sat. aq. $\mathrm{NaHCO}_{3}$ solution $(2 \times 15 \mathrm{~mL})$ and $\mathrm{HCl}(1 \mathrm{M}, 2 \times 15 \mathrm{~mL})$. The organic layer was 
tried over $\mathrm{Na}_{2} \mathrm{SO}_{4}$ and the solvent was removed under reduced pressure. The residue was recrystallized from toluene. The product [2.58 g, 92\%, $11.6 \mathrm{mmol}$ (Lit.9: 95\%)] was obtained as a white solid. It represented a mixture of two rotamers $[(Z, S, S)-\mathbf{1 8}:(E, S, S)-\mathbf{1 8}=67: 33]$. This ratio was determined by integrating the $3-\mathrm{H}_{3}$ signals [at $\delta=1.01(Z)-\mathbf{1 8}$ and $\left.1.22(E)-\mathbf{1 8} \mathrm{ppm}\right]$.

The enantiomer $(R, R)-\mathbf{1 8}$ was obtained analogously in $92 \%$ yield.

${ }^{1} \mathrm{H}$ NMR $\left(300.13 \mathrm{MHz}, \mathrm{C}_{6} \mathrm{D}_{6} / \mathrm{C}_{6} \mathrm{H}_{6}, \mathrm{DsBrNo} 07-31180,7.11 .2019 ; 67: 33\right.$ rotamer ration, asterisk denotes $E$-rotamer peaks): $\delta=0.58\left(\mathrm{~d}, J_{1^{\prime}-\mathrm{CH}_{3}, 1^{\prime}}=6.8 \mathrm{~Hz}, 3 \mathrm{H}, 1^{\prime}-\mathrm{CH}_{3}\right)^{*}, 0.95\left(\mathrm{~d}, J_{1^{\prime}-}\right.$ $\left.\mathrm{CH}_{3}, 1^{\prime}=7.0 \mathrm{~Hz}, 3 \mathrm{H}, 1^{\prime}-\mathrm{CH}_{3}\right), 1.01\left(\mathrm{t}, J_{3,2}=7.4 \mathrm{~Hz}, 3 \mathrm{H}, 3-\mathrm{H}_{3}\right), 1.22\left(\mathrm{t}, J_{3,2}=7.3 \mathrm{~Hz}, 3 \mathrm{H}, 3-\mathrm{H}_{3}\right)^{*}$, 1.67-1.86 (m, 2H, 2- $\left.\mathrm{H}_{2}\right), 2.13\left(\mathrm{~s}, 3 \mathrm{H}, N-\mathrm{CH}_{3}\right), 2.42-2.54\left(\mathrm{~m}, 1 \mathrm{H}, 1^{\prime}-\mathrm{H}\right)^{*}, 2.83\left(\mathrm{~s}, 3 \mathrm{H}, N-\mathrm{CH}_{3}\right)^{*}$, $3.73\left(\mathrm{~m}_{\mathrm{c}}, 1 \mathrm{H}, 2^{\prime}-\mathrm{H}\right)^{*}, 4.20-4.35$ (m, 1H, 1'-H), 4.35 (t, J = 7.0 Hz, 1H, 2'-H), 4.94 (br. s, 1H, 2'$\mathrm{OH}), 7.04-7.35\left(\mathrm{~m}, 5 \mathrm{H}, \mathrm{Ar}-\mathrm{H}_{5}\right)$.

Specific rotation $(R, R)-\mathbf{1 8}[a]_{D}^{20}=-111.5^{\circ}\left(\mathrm{c}=1.0\right.$ in $\left.\mathrm{CHCl}_{3}\right)$.

Specific rotation $(S, S)-\mathbf{1 8}[a]_{D}^{20}=+113.3^{\circ}\left(\mathrm{c}=1.0\right.$ in $\left.\mathrm{CHCl}_{3}\right)$.

\footnotetext{
${ }^{9}$ A. G. Myers, B. H. Yang, H. Chen, L. McKinstry, D. J. Kopecky, J. L. Gleason, J. Am. Chem. Soc. 1997, 119, 6496-6511.
} 


\section{$(1 Z, 2 R)-N-[(1 ' S, 2$ ' $S)-2$ '-Hydroxy-2'-phenylpropan-1'-yl]- $N, 2$-dimethylbutanamide}

$(Z, S, S, R)-26$

in an 82:18 mixture with

(1E,2R)-N-[(1'S,2'S)-2'-Hydroxy-2'-phenylpropan-1'-yl]- $N, 2$-dimethylbutanamide $(E, S, S, R)-26$

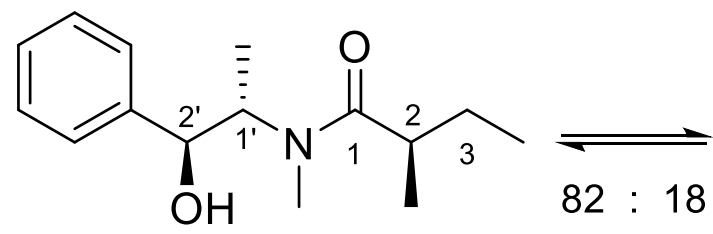

$(Z, S, S, R)-\mathbf{2 6}$<smiles>CC[C@H](C)C(=O)N(C)[C@@H](C)[C@H](O)c1ccccc1</smiles>

$(E, S, S, R)-26$

A solution of $n \mathrm{BuLi}$ (1.40 M in hexane, $33.8 \mathrm{~mL}, 47.3 \mathrm{mmol}, 2.1$ eq.) was added to a suspension of $\mathrm{LiCl}$ (6.68 g, $158 \mathrm{mmol}, 7.0$ eq.) and diisopropylamine (7.30 mL, $5.26 \mathrm{~g}, 51.9 \mathrm{mmol}, 2.3 \mathrm{eq}$.) in THF $(22 \mathrm{~mL})$ at $-78^{\circ} \mathrm{C}$. The mixture was warmed to $0^{\circ} \mathrm{C}$ briefly and was cooled again to $78^{\circ} \mathrm{C}$. An ice-cooled solution of the amide $(S, S)-18(5.00 \mathrm{~g}, 22.6 \mathrm{mmol})$ in THF $(55 \mathrm{~mL})$ was added. The mixture stirred at $-78^{\circ} \mathrm{C}$ for $1 \mathrm{~h}$, at $0^{\circ} \mathrm{C}$ for $15 \mathrm{~min}$ and at $25^{\circ} \mathrm{C}$ for $5 \mathrm{~min}$. The mixture was cooled to $0^{\circ} \mathrm{C}$ and EtI $(7.24 \mathrm{~mL}, 14.1 \mathrm{~g}, 90.0 \mathrm{mmol}, 4.0$ eq.) was added. The mixture stirred at $25^{\circ} \mathrm{C}$ for $15 \mathrm{~h}$. The mixture was diluted with sat. aq. $\mathrm{NH}_{4} \mathrm{Cl}$ solution $(50 \mathrm{~mL})$, the layers were separated and the aq. layer was extracted with AcOEt $(4 \times 50 \mathrm{~mL})$. The combined organic layers were dried over $\mathrm{MgSO}_{4}$ and concentrated under reduced pressure. Purification by flash chromatography $\left(c \mathrm{C}_{6} \mathrm{H}_{12}\right.$ :AcOEt $\left.=2: 1,8 \mathrm{~cm}, 15 \mathrm{~cm}, 100 \mathrm{~mL}\right)$ afforded $(S, S, R)-\mathbf{2 6}\left[5.30 \mathrm{~g}, 94 \%, 44.5 \mathrm{mmol}\left(\right.\right.$ Lit. $\left.\left.^{10}: 88 \%\right)\right]$ as a pale yellow oil. It represented a mixture of two rotamers $[(Z, S, S, R)-\mathbf{2 6}$ : $(E, S, S, R)-\mathbf{2 6}=82: 18]$. This ratio was determined by integrating the $N-\mathrm{CH}_{3}$ signals [at $\delta=2.85(Z)-26$ and $2.91(E)-26 \mathrm{ppm}$ ].

$\mathrm{R}_{\mathrm{f}}\left(c \mathrm{C}_{6} \mathrm{H}_{12}: \mathrm{AcOEt}=2: 1\right)=0.23$

${ }^{1} \mathbf{H}$ NMR $\left(300.13 \mathrm{MHz}, \mathrm{CDCl}_{3} / \mathrm{CHCl}_{3}\right.$, DsBrNo21-30870, 21.11.2019; 82:18 rotamer ration, asterisk denotes $E$-rotamer peaks): $\delta=0.84$ (t, $\left.J_{4,3}=7.4 \mathrm{~Hz}, 3 \mathrm{H}, 4-\mathrm{H}_{3}\right), 0.90\left(\mathrm{t}, J_{4,3}=7.1 \mathrm{~Hz}\right.$, $\left.3 \mathrm{H}, 4-\mathrm{H}_{3}\right)^{*}, 1.02\left(\mathrm{~d}, J_{1^{\prime}-\mathrm{CH}_{3}, 1^{\prime}}=6.8 \mathrm{~Hz}, 3 \mathrm{H}, 1^{\prime}-\mathrm{CH}_{3}\right)^{*}, 1.09\left(\mathrm{~d}, J_{2-\mathrm{CH}_{3}, 2}=6.7 \mathrm{~Hz}, 3 \mathrm{H}, 2-\mathrm{CH}_{3}\right)$,

\footnotetext{
${ }^{10}$ F. Bellotta, M. V. D`Auria, V. Sepe, A. Zampella, Tetrahedron 2009, 65, 3659-3663.
} 
$1.14\left(\mathrm{~d}, J_{1^{\prime}-\mathrm{CH}_{3}, 1^{\prime}}=7.0 \mathrm{~Hz}, 3 \mathrm{H}, 1^{\prime}-\mathrm{CH}_{3}\right), 1.32-1.43\left(\mathrm{~m}, 1 \mathrm{H}, 3-\mathrm{H}^{\mathrm{A}}\right), 1.56-1.70\left(\mathrm{~m}, 1 \mathrm{H}, 3-\mathrm{H}^{\mathrm{B}}\right), 2.53$ $\left(\mathrm{sx}, J_{2,3}=J_{2,2-\mathrm{CH}_{3}}=7.0 \mathrm{~Hz}, 1 \mathrm{H}, 2-\mathrm{H}\right), 2.85\left(\mathrm{~s}, 3 \mathrm{H}, N-\mathrm{CH}_{3}\right), 2.91\left(\mathrm{~s}, 3 \mathrm{H}, N-\mathrm{CH}_{3}\right)^{*}, 4.06-4.15(\mathrm{~m}$, $\left.1 \mathrm{H}, 1^{\prime}-\mathrm{H}\right)^{*}, 4.42$ (br. s, $\left.1 \mathrm{H}, 1^{\prime}-\mathrm{H}\right), 4.62\left(\mathrm{t}, J_{2^{\prime}, 1^{\prime}}=J_{2^{\prime}, 2^{\prime}-\mathrm{OH}}=7.2 \mathrm{~Hz}, 1 \mathrm{H}, 2^{\prime}-\mathrm{H}\right), 7.22-7.38(\mathrm{~m}, 5 \mathrm{H}$, Ar- $\left.\mathrm{H}_{5}\right)$.

(2R)-2-Methylbutanol [(R)-19]

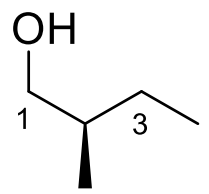

$(R)-19$

A solution of $n \mathrm{BuLi}$ (2.25 $\mathrm{M}$ in hexane, $18.5 \mathrm{~mL}, 41.6 \mathrm{mmol}, 3.9$ eq.) was added to a solution of diisopropylamine $\left(6.35 \mathrm{~mL}, 4.55 \mathrm{~g}, 44.8 \mathrm{mmol}, 4.2 \mathrm{eq}\right.$.) in THF $(12.2 \mathrm{~mL})$ at $-78^{\circ} \mathrm{C}$. After $10 \mathrm{~min}$ the solution was warmed to $0^{\circ} \mathrm{C}$ and held this temperature for $10 \mathrm{~min} . \mathrm{BH}_{3} \mathrm{NH}_{3}(1.32 \mathrm{~g}$, $42.6 \mathrm{mmol}, 4.0$ eq.) was added in one portion and the suspension stirred at $0^{\circ} \mathrm{C}$ for $15 \mathrm{~min}$ and at $25^{\circ} \mathrm{C}$ for $15 \mathrm{~min}$. A solution of the amide $(S, S, R)-26(2.65 \mathrm{~g}, 10.6 \mathrm{mmol})$ in THF $(20 \mathrm{~mL})$ was added at $0^{\circ} \mathrm{C}$. The mixture was warmed to $25^{\circ} \mathrm{C}$ and held this temperature for $2 \mathrm{~h}$. Then the mixture was cooled to $0^{\circ} \mathrm{C}$ and the excess hydride was quenched with $\mathrm{HCl}(3 \mathrm{M}, 20 \mathrm{~mL})$. The solution stirred at $0^{\circ} \mathrm{C}$ for $2 \mathrm{~h}$. The organic layer was separated and the aq. layer was extracted with $\mathrm{Et}_{2} \mathrm{O}(3 \times 40 \mathrm{~mL})$. The combined organic layers were washed with $\mathrm{HCl}(3 \mathrm{M}$, $20 \mathrm{~mL})$, aq. $\mathrm{NaOH}(2 \mathrm{M}, 20 \mathrm{~mL})$ and brine $(20 \mathrm{~mL})$. The combined organic layers were dried over $\mathrm{MgSO}_{4}$ and concentrated under reduced pressure. Purification by flash chromatography (PE 30/50:Et $2 \mathrm{O}=3: 1,3.5 \mathrm{~cm}, 15 \mathrm{~cm}, 30 \mathrm{~mL})$ afforded $(R)-\mathbf{1 9}\left(720 \mathrm{mg}, 76 \%, 8.14\right.$ mmol (Lit. ${ }^{10}$ : $85 \%)$ as a colorless liquid.

$\mathbf{R}_{\mathbf{f}}\left(\mathrm{PE} 30 / 50: \mathrm{Et}_{2} \mathrm{O}=3: 1\right)=0.42$

${ }^{1} \mathbf{H}$ NMR (300.13 MHz, $\mathrm{CDCl}_{3} / \mathrm{CHCl}_{3}$, DsBrSe26-30160, 26.9.2019): $\delta=0.90$ (t, $J_{4,3}=7.3 \mathrm{~Hz}$, $\left.3 \mathrm{H}, 4-\mathrm{H}_{3}\right), 0.91\left(\mathrm{~d}, J_{2-\mathrm{CH}_{3}, 2}=6.6 \mathrm{~Hz}, 3 \mathrm{H}, 2-\mathrm{CH}_{3}\right), 1.06-1.28\left(\mathrm{~m}, 1 \mathrm{H}, 3-\mathrm{H}^{\mathrm{A}}\right), 1.33$ (br. s, $1 \mathrm{H}, 1-$ $\mathrm{OH}), 1.37-1.61\left(\mathrm{~m}, 2 \mathrm{H}, 3-\mathrm{H}^{\mathrm{B}}, 2-\mathrm{H}\right), \mathrm{AB}$ signal $\left(\delta_{\mathrm{A}}=3.42, \delta_{\mathrm{B}}=3.51, J_{\mathrm{AB}}=9.9 \mathrm{~Hz}\right.$ in addition split by $\left.J_{1-\mathrm{H}^{\mathrm{A}}, 2}=5.8 \mathrm{~Hz}, J_{1-\mathrm{H}^{\mathrm{B}}, 2}=6.3 \mathrm{~Hz}, 2 \mathrm{H}, 1-\mathrm{H}_{2}\right)$. 
and

\section{(2S)-2-Methylbutyl 4-Methylbenzenesulfonate [(S)-27]}

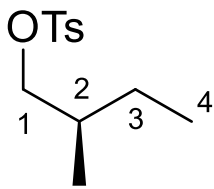

$(R)-27$

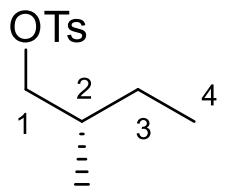

$(S)-27$

(2R)-2-Methylbutanol [(R)-19] (500 $\mathrm{mg}, 5.70 \mathrm{mmol})$ was dissolved in pyridine $(0.9 \mathrm{~mL})$ and cooled to $0^{\circ} \mathrm{C}$. Then $\mathrm{TsCl}(1.12 \mathrm{~g}, 5.80 \mathrm{mmol}, 1.0$ eq.) was added slowly. The mixture was stirred at $0^{\circ} \mathrm{C}$ for $12 \mathrm{~h}$. The mixture was deluded with $\mathrm{H}_{2} \mathrm{O}(3 \mathrm{~mL})$, the organic layer was separated and aq. layer was extracted with $\mathrm{Et}_{2} \mathrm{O}(3 \times 5 \mathrm{~mL})$. The combined organic layers were washed with sat. aq. $\mathrm{CuSO}_{4}$ solution $(5 \mathrm{~mL}), \mathrm{H}_{2} \mathrm{O}(5 \mathrm{~mL})$ and brine $(5 \mathrm{~mL})$. The combined organic layers were dried over $\mathrm{MgSO}_{4}$ and concentrated under reduced pressure. The product [1.15 g, 83\%, $4.73 \mathrm{mmol}$ (Lit. ${ }^{11}$ : 99\%)] was afforded as colorless oil.

The enantiomer $(S)-27$ was obtained analogously in $83 \%$ yield.

${ }^{1} \mathbf{H}$ NMR $\left(300.13 \mathrm{MHz}, \mathrm{CDCl}_{3} / \mathrm{CHCl}_{3}\right.$, sample contained $\mathrm{H}_{2} \mathrm{O}$, trace amounts of Acetone and unknown impurity $\mathrm{m}$ at 7.67 and $\mathrm{s}$ at 8.62, DsBrOc21-30430, 21.10.2014): $\delta=0.83$ (t, $\left.J_{4,3}=7.4 \mathrm{~Hz}, 3 \mathrm{H}, 4-\mathrm{H}_{3}\right), 0.88\left(\mathrm{~d}, J_{2-\mathrm{CH}_{3}, 2}=6.8 \mathrm{~Hz}, 3 \mathrm{H}, 2-\mathrm{CH}_{3}\right), 1.07-1.23\left(\mathrm{~m}, 1 \mathrm{H}, 3-\mathrm{H}^{\mathrm{A}}\right), 1.32-$ $1.47\left(\mathrm{~m}, 1 \mathrm{H}, 3-\mathrm{H}^{\mathrm{B}}\right), 1.70\left(\mathrm{sx}, J_{2,1}=J_{2,3}=6.8 \mathrm{~Hz}, 1 \mathrm{H}, 2-\mathrm{H}\right), 2.45\left(\mathrm{~s}, 3 \mathrm{H}, \mathrm{Ar}-\mathrm{CH}_{3}\right), \mathrm{AB}$ signal $\left(\delta_{\mathrm{A}}=3.82, \delta_{\mathrm{B}}=3.89, J_{\mathrm{AB}}=9.4 \mathrm{~Hz}\right.$, in addition split by $J_{1-\mathrm{H}^{\mathrm{A}}, 2}=6.4 \mathrm{~Hz}, J_{1-\mathrm{H}^{\mathrm{B}}, 2}=5.9 \mathrm{~Hz}, 2 \mathrm{H}, 1-$ $\left.\mathrm{H}_{2}\right), 7.34\left(\mathrm{~d}, J=8.0 \mathrm{~Hz}, 2 \mathrm{H}, \mathrm{Ar}-\mathrm{H}_{2}\right), 7.79$ (d, $\left.J=8.3 \mathrm{~Hz}, 2 \mathrm{H}, \mathrm{Ar}-\mathrm{H}_{2}\right)$.

Specific rotation $(S)-27[a]_{D}^{20}=+8.1^{\circ}\left(\mathrm{c}=1.0\right.$ in $\left.\mathrm{CHCl}_{3}\right)$.

Specific rotation $(R)-27[a]_{D}^{20}=-8.3^{\circ}\left(\mathrm{c}=1.0\right.$ in $\left.\mathrm{CHCl}_{3}\right)$.

\footnotetext{
${ }^{11}$ K. Mori, A. Kamada, M. Kido, Liebigs Ann. Chem. 1991, 775-781.
} 
and

(2S)-1-Iodo-2-methylbutane $[(S)-28]$

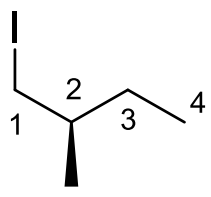

$(R)-28$

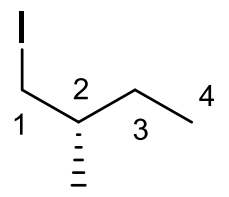

$(S)-28$

The tosylate $(R)-27(1.10 \mathrm{~g}, 4.60 \mathrm{mmol})$ was dissolved in DMF $(5.0 \mathrm{~mL})$. NaI $(1.50 \mathrm{~g}$, $10.0 \mathrm{mmol}, 2.2 \mathrm{eq}$.) was added and the mixture was stirred at $60^{\circ} \mathrm{C}$ for $1.5 \mathrm{~h}$ under light exclusion. $\mathrm{H}_{2} \mathrm{O}(5 \mathrm{~mL})$ was added, the organic layer was separated and the aq. layer was extracted with pentane $(3 \times 5 \mathrm{~mL})$. The combined organic layers were washed with $\mathrm{H}_{2} \mathrm{O}(2 \mathrm{~mL})$ and brine $(2 \mathrm{~mL})$, tried over $\mathrm{MgSO}_{4}$ and concentrated under reduced pressure. Distillation (bp.25mbar: $48^{\circ} \mathrm{C}$ ) afforded (R)-28 [530 mg, 58\%, $2.67 \mathrm{mmol}$ (Lit. ${ }^{12}: 89 \%$ )] as a colorless liquid.

The enantiomer $(S)$-28 was obtained analogously in $68 \%$ yield.

${ }^{1} \mathbf{H}$ NMR (300.13 MHz, $\mathrm{CDCl}_{3} / \mathrm{CHCl}_{3}$, sample contained $\mathrm{H}_{2} \mathrm{O}$, DsBrOc22-30520, 22.10.2014): $\delta=0.83\left(\mathrm{t}, J_{4,3}=7.3 \mathrm{~Hz}, 3 \mathrm{H}, 4-\mathrm{H}_{3}\right), 0.91\left(\mathrm{~d}, J_{2-\mathrm{CH}_{3}, 2}=6.2 \mathrm{~Hz}, 3 \mathrm{H}, 2-\mathrm{CH}_{3}\right), 1.11-1.40(\mathrm{~m}, 3 \mathrm{H}$, $\left.3-\mathrm{H}_{2}, 2-\mathrm{H}\right), \mathrm{AB}$ signal $\left(\delta_{\mathrm{A}}=3.17, \delta_{\mathrm{B}}=3.23, J_{\mathrm{AB}}=9.6 \mathrm{~Hz}\right.$ in addition split by $J_{1-\mathrm{H}^{\mathrm{A}}, 2}=4.6 \mathrm{~Hz}$, $\left.J_{1-\mathrm{H}^{\mathrm{B}}, 2}=5.7 \mathrm{~Hz}, 2 \mathrm{H}, 1-\mathrm{H}_{2}\right)$.

Specific rotation $(S)-28[a]_{D}^{20}=+5.6^{\circ}\left(\mathrm{c}=1.0\right.$ in $\left.\mathrm{CHCl}_{3}\right)$.

Specific rotation $(R)-28[a]_{D}^{20}=-5.1^{\circ}\left(\mathrm{c}=1.0\right.$ in $\left.\mathrm{CHCl}_{3}\right)$.

IR (KBr): $\tilde{v}=3495,2985,2205,2055,1960,1890,1820,1735,1510,1465,1275,1260,1210$, $1160,1035,955,895,825,765,750,705,510,500 \mathrm{~cm}^{-1}$.

\footnotetext{
${ }^{12}$ K. Akasaka, S. Tamogami, R. W. Beeman, K. Mori, Tetrahedron 2011, 67, 201-209.
} 
Christian Drescher, Morris Keller, Olivier Potterat, Matthias Hamburger and Reinhard Brückner, Structure-Elucidating Total Synthesis of the (Polyenoyl)tetramic Acid Militarinone C

$(1 Z, 2 R, 4 R)-N-[(1 ' S, 2$ ' $S)-2$ '-Hydroxy-1'-methyl-2'-phenylethyl]- $N$-methyl-2,4dimethylhexanamide $[(Z, S, S, R, R)-15]$

in an 85:15 mixture with

$(1 E, 2 R, 4 R)-N-[(1 ' S, 2 ' S)-2 '-H y d r o x y-1 '-m e t h y l-2 '$-phenylethyl]- $N$-methyl-2,4dimethylhexanamide $[(E, S, S, R, R)-15]$

and

$(1 Z, 2 S, 4 S)-N-\left[\left(1^{\prime} R, 2^{\prime} R\right)-2 '-H y d r o x y-1 '-m e t h y l-2 '-p h e n y l e t h y l\right]-N$-methyl-2,4dimethylhexanamide $[(Z, R, R, S, S)-15]$

in an 85:15 mixture with

$(1 E, 2 S, 4 S)-N-\left[\left(1^{\prime} R, 2^{\prime} R\right)-2 '-H y d r o x y-1 '-m e t h y l-2 '\right.$-phenylethyl]- $N$-methyl-2,4dimethylhexanamide $[(E, R, R, S, S)-15]$

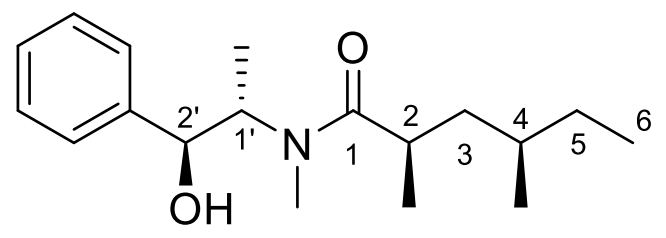

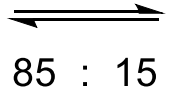<smiles>CC[C@H](C)C[C@H](C)C(=O)N(C)[C@@H](C)[C@H](O)c1ccccc1</smiles>

$(Z, S, S, R, R)-15$

$(E, S, S, R, R)-15$<smiles>CC[C@H](C)C[C@H](C)C(=O)N(C)[C@@H](C)[C@H](O)c1ccccc1</smiles>

$85: 15$<smiles>CC[C@H](C)C[C@@H](C)C(=O)N(C)[C](C)[C@H](O)c1ccccc1</smiles>

$(Z, R, R, S, S)-15$

$(E, R, R, S, S)-15$

A solution of $n \mathrm{BuLi}$ (2.33 $\mathrm{M}$ in hexane, $4.43 \mathrm{~mL}, 10.4 \mathrm{mmol}, 4.0$ eq.) was added to a suspension of $\mathrm{LiCl}$ (1.39 g, $32.8 \mathrm{mmol} 12.7$ eq.) and diisopropylamine (1.12 g, $11.5 \mathrm{mmol}, 4.3 \mathrm{eq}$.) in THF $(7.5 \mathrm{~mL})$ at $-78^{\circ} \mathrm{C}$. The mixture was warmed to $0^{\circ} \mathrm{C}$ briefly and was cooled again to $-78^{\circ} \mathrm{C}$. An 
ice-cooled solution of the amide $(S, S)-\mathbf{1 8}(1.20 \mathrm{~g}, 5.40 \mathrm{mmol}, 2.1$ eq.) in THF (16.3 mL) was added. The mixture stirred at $-78^{\circ} \mathrm{C}$ for $1 \mathrm{~h}$, at $0^{\circ} \mathrm{C}$ for $15 \mathrm{~min}$ and at $25^{\circ} \mathrm{C}$ for $5 \mathrm{~min}$. The mixture was cooled to $0^{\circ} \mathrm{C}$ and the iodide $(R)-28(500 \mathrm{mg}, 2.60 \mathrm{mmol})$ was added. The mixture stirred at $25^{\circ} \mathrm{C}$ for $24 \mathrm{~h}$. The mixture was diluted with sat. aq. $\mathrm{NH}_{4} \mathrm{Cl}$ solution $(20 \mathrm{~mL})$, the layers were separated and the aq. layer was extracted with AcOEt $(4 \times 40 \mathrm{~mL})$. The combined organic layers were dried over $\mathrm{MgSO}_{4}$ and concentrated under reduced pressure. Purification by flash chromatography $\left(c \mathrm{C}_{6} \mathrm{H}_{12}: \mathrm{AcOEt}=2: 1,2.5 \mathrm{~cm}, 15 \mathrm{~cm}, 20 \mathrm{~mL}\right)$ afforded $(S, S, R, R)-15$ [640 mg, 88\%, 2.27 mmol (Lit. $\left.\left.{ }^{9}: 95 \%\right)\right]$ as a pale yellow oil. It represented a mixture of two rotamers $[(Z, S, S, R, R)-\mathbf{1 5}:(E, S, S, R, R)-\mathbf{1 5}=85: 15]$. This ratio was determined by integrating the $N-\mathrm{CH}_{3}$ signals [at $\delta=2.85(Z)-\mathbf{1 5}$ and $\left.2.90(E)-\mathbf{1 5} \mathrm{ppm}\right]$.

The enantiomer $(R, R, S, S)$-15 was obtained analogously in 94\% yield.

$\mathbf{R}_{\mathbf{f}}\left(c \mathrm{C}_{6} \mathrm{H}_{12}: \mathrm{AcOEt}=1: 1\right)=0.45$

${ }^{1} \mathbf{H}$ NMR $\left(300.13 \mathrm{MHz}, \mathrm{CDCl}_{3} / \mathrm{CHCl}_{3}\right.$, DsBrNo07-31190, 7.11.2019, 85:15 rotamer ration, asterisk denotes E-rotamer peaks): $\delta=0.77\left(\mathrm{~d}, J_{4-\mathrm{CH}_{3}, 4}=6.5 \mathrm{~Hz}, 3 \mathrm{H}, 4-\mathrm{CH}_{3}\right), 0.86$ (t, $\left.J_{6,5}=7.1 \mathrm{~Hz}, 3 \mathrm{H}, 6-\mathrm{H}_{3}\right), 0.98-1.08\left(\mathrm{~m}, 1 \mathrm{H}, 5-\mathrm{H}^{\mathrm{A}}\right), 1.07\left(\mathrm{~d}, J_{2-\mathrm{CH}_{3}, 2}=6.8 \mathrm{~Hz}, 3 \mathrm{H}, 2-\mathrm{CH}_{3}\right), 1.15$ $\left(\mathrm{d}, J_{1^{\prime}-\mathrm{CH}_{3}, 1^{\prime}}=7.0 \mathrm{~Hz}, 3 \mathrm{H}, 1^{\prime}-\mathrm{CH}_{3}\right), 1.21-1.40\left(\mathrm{~m}, 1 \mathrm{H}, 5-\mathrm{H}^{\mathrm{B}}\right), 1.21-1.40\left(\mathrm{~m}, 2 \mathrm{H}, 3-\mathrm{H}_{2}\right), 1.71\left(\mathrm{~m}_{\mathrm{c}}\right.$, $1 \mathrm{H}, 4-\mathrm{H}), 1.93\left(\mathrm{~m}_{\mathrm{c}}, 1 \mathrm{H}, 4-\mathrm{H}\right)^{*}, 2.61-2.76(\mathrm{~m}, 1 \mathrm{H}, 2-\mathrm{H}), 2.85\left(\mathrm{~s}, 3 \mathrm{H}, N-\mathrm{CH}_{3}\right), 2.90(\mathrm{~s}, 3 \mathrm{H}, N-$ $\left.\mathrm{CH}_{3}\right)^{*}, 3.02\left(\mathrm{~m}_{\mathrm{c}}, 1 \mathrm{H}, 2-\mathrm{H}\right)^{*}, 4.11\left(\mathrm{~m}, 1 \mathrm{H}, 1^{\prime}-\mathrm{H}\right)^{*}, 4.38$ (br. s, 1H, 1'-H), 4.61 (t, $J_{2^{\prime}, 1^{\prime}}=J_{2^{\prime}, 2^{\prime}-\mathrm{OH}}$ $=6.6 \mathrm{~Hz}, 1 \mathrm{H}, 2 \mathrm{\prime}-\mathrm{H}), 7.21-7.38\left(\mathrm{~m}, 5 \mathrm{H}, \mathrm{Ar}-\mathrm{H}_{5}\right)$.

Specific rotation $(R, R, S, S)-15[a]_{D}^{20}=-65.9^{\circ}\left(\mathrm{c}=1.0\right.$ in $\left.\mathrm{CHCl}_{3}\right)$.

Specific rotation $(S, S, R, R)-15[a]_{D}^{20}=+64.3^{\circ}\left(\mathrm{c}=1.0\right.$ in $\left.\mathrm{CHCl}_{3}\right)$.

HRMS (ESI) m/z: [M-H] $]^{+}$Calcd for $\mathrm{C}_{18} \mathrm{H}_{28} \mathrm{O}_{2} \mathrm{~N}$ 290.2126; Found 290.2124

IR (KBr): $\tilde{v}=3380,3065,2960,2930,2875,1620,1455,1410,1375,1235,1305,1200,1110$, $1085,1050,755,700,610,515 \mathrm{~cm}^{-1}$. 
and

\section{$(2 S, 4 S)-2,4-D i m e t h y l h e x a n-1-o l[(S, S)-29]$}<smiles>CC[C@@H](C)CC(C)CO</smiles>

$(R, R)-29$

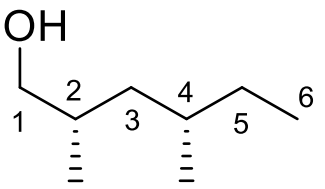

$(S, S)-29$

A solution of $n \mathrm{BuLi}$ (2.44 $\mathrm{M}$ in hexane, $3.13 \mathrm{~mL}, 7.60 \mathrm{mmol}, 3.9$ eq.) was added to a solution of diisopropylamine ( $840 \mathrm{mg}, 8.20 \mathrm{mmol}, 4.2 \mathrm{eq}$.) in $\mathrm{THF}(2.2 \mathrm{~mL})$ at $-78^{\circ} \mathrm{C}$. After $10 \mathrm{~min}$ the solution was warmed to $0^{\circ} \mathrm{C}$ and held this temperature for $10 \mathrm{~min}$. $\mathrm{BH}_{3} \mathrm{NH}_{3}(240 \mathrm{mg}$, $7.79 \mathrm{mmol}, 4.0$ eq.) was added in one portion and the suspension stirred at $0^{\circ} \mathrm{C}$ for $15 \mathrm{~min}$ and at $25^{\circ} \mathrm{C}$ for $15 \mathrm{~min}$. A solution of the amide $(S, S, R, R)-15(570 \mathrm{mg}, 2.01 \mathrm{mmol})$ in THF $(5 \mathrm{~mL})$ was added at $0^{\circ} \mathrm{C}$. The mixture was warmed to $25^{\circ} \mathrm{C}$ and held this temperature for $2 \mathrm{~h}$. Then the mixture was cooled to $0^{\circ} \mathrm{C}$ and the excess hydride quenched with $\mathrm{HCl}(3 \mathrm{M}, 10 \mathrm{~mL})$. The solution stirred at $0^{\circ} \mathrm{C}$ for $2 \mathrm{~h}$. The organic layer was separated and the aq. layer was extracted with $\mathrm{Et}_{2} \mathrm{O}(3 \times 20 \mathrm{~mL})$. The combined organic layers were washed with $\mathrm{HCl}(3 \mathrm{M}, 10 \mathrm{~mL})$, aq. $\mathrm{NaOH}(2 \mathrm{M}, 10 \mathrm{~mL})$ and brine $(10 \mathrm{~mL})$. The combined organic layers were dried over $\mathrm{MgSO}_{4}$ and concentrated under reduced pressure. Purification by flash chromatography (PE 30/50: $\mathrm{Et}_{2} \mathrm{O}$ $=3: 1,2.0 \mathrm{~cm}, 10 \mathrm{~cm}, 15 \mathrm{~mL})$ afforded $(R, R)-29$ [189 mg, 74\%, $1.48 \mathrm{mmol}\left(\right.$ Lit. $\left.^{10}: 88 \%\right)$ ] as a colorless liquid.

The enantiomer $(S, S)-\mathbf{2 9}$ was obtained analogously in $80 \%$ yield.

$\mathbf{R}_{\mathbf{f}}\left(\mathrm{PE} 30 / 50: \mathrm{Et}_{2} \mathrm{O}=3: 1\right)=0.39$

${ }^{1} \mathbf{H}$ NMR $\left(500.10 \mathrm{MHz}, \mathrm{CDCl}_{3} / \mathrm{CHCl}_{3}\right.$, sample contained 4 weight-\% of Et $2 \mathrm{O}$, DsBrJa26-5010, 27.1.2015): $\delta=0.88\left(\mathrm{~d}, J_{4-\mathrm{CH}_{3}, 4}=6.4 \mathrm{~Hz}, 3 \mathrm{H}, 4-\mathrm{CH}_{3}\right), 0.89\left(\mathrm{t}, J_{6,5}=7.4 \mathrm{~Hz}, 3 \mathrm{H}, 6-\mathrm{H}_{3}\right), 0.90-$ $0.96\left(\mathrm{~m}, 1 \mathrm{H}, 3-\mathrm{H}^{\mathrm{A}}\right), 0.93\left(\mathrm{~d}, J_{2-\mathrm{CH}_{3}, 2}=6.7 \mathrm{~Hz}, 3 \mathrm{H}, 2-\mathrm{CH}_{3}\right), 1.03-1.14\left(\mathrm{~m}, 1 \mathrm{H}, 5-\mathrm{H}^{\mathrm{A}}\right), 1.21-1.49$ $\left(\mathrm{m}, 1 \mathrm{H}, 3-\mathrm{H}^{\mathrm{B}}\right), 1.21-1.49\left(\mathrm{~m}, 1 \mathrm{H}, 5-\mathrm{H}^{\mathrm{B}}\right), 1.21-1.49(\mathrm{~m}, 1 \mathrm{H}, 4-\mathrm{H}), 1.72\left(\mathrm{sx}, J_{2,1}=J_{2,3}=6.5 \mathrm{~Hz}\right.$, $1 \mathrm{H}, 2-\mathrm{H}), 3.38\left(\mathrm{dd}, J_{1-\mathrm{H}^{\mathrm{A}}, 2}=6.7 \mathrm{~Hz}, J_{1-\mathrm{H}^{\mathrm{A}}, \mathrm{OH}}=10.5 \mathrm{~Hz}, 1 \mathrm{H}, 1-\mathrm{H}^{\mathrm{A}}\right), 3.52\left(\mathrm{dd}, J_{1-\mathrm{H}^{\mathrm{B}}, 2}=5.2 \mathrm{~Hz}, J_{1-}\right.$ $\left.\mathrm{H}^{\mathrm{B}}, \mathrm{OH}=10.5 \mathrm{~Hz}, 1-\mathrm{H}^{\mathrm{B}}\right)$. 
Christian Drescher, Morris Keller, Olivier Potterat, Matthias Hamburger and Reinhard Brückner, Structure-Elucidating Total Synthesis of the (Polyenoyl)tetramic Acid Militarinone C

${ }^{13} \mathrm{C}$ NMR (125.75 MHz, $\mathrm{CDCl}_{3} / \mathrm{CHCl}_{3}$, sample contained $\mathrm{Et}_{2} \mathrm{O}$, DsBrJa26-5014, 27.1.2015): $\delta=11.2(\mathrm{C}-6), 17.4\left(2-\mathrm{CH}_{3}\right), 19.9\left(4-\mathrm{CH}_{3}\right), 29.2(\mathrm{C}-5), 31.7(\mathrm{C}-4), 33.2(\mathrm{C}-2), 40.7(\mathrm{C}-3), 68.5$ $(\mathrm{C}-1)$.

edHSQC [,,short-range H,C-COSY“, $500.10 \mathrm{MHz} / 125.75 \mathrm{MHz}, \mathrm{CDCl}_{3} ; \delta\left({ }^{1} \mathrm{H}\right) \leftrightarrow \delta\left({ }^{13} \mathrm{C}\right): 0.88$ $\left(\mathrm{d}, 4-\mathrm{CH}_{3}\right) \leftrightarrow 19.9\left(4-\mathrm{CH}_{3}\right), 0.89\left(\mathrm{t}, 6-\mathrm{H}_{3}\right) \leftrightarrow 11.3(\mathrm{C}-6), 0.90-0.96\left(\mathrm{~m}, 3-\mathrm{H}^{\mathrm{B}}\right) \leftrightarrow 40.7(\mathrm{C}-3)$, $0.93\left(\mathrm{~d}, 2-\mathrm{CH}_{3}\right) \leftrightarrow 17.4\left(2-\mathrm{CH}_{3}\right), 1.03-1.14(\mathrm{~m}, 5-\mathrm{H}) \leftrightarrow 29.2(\mathrm{C}-5), 1.21-1.49\left(\mathrm{~m}, 3-\mathrm{H}^{\mathrm{A}}\right) \leftrightarrow$ 40.7 (C-3), 1.21-1.49 (m, 5- $\left.\mathrm{H}^{\mathrm{A}}\right) \leftrightarrow 29.2(\mathrm{C}-5), 1.21-1.49(\mathrm{~m}, 4-\mathrm{H}) \leftrightarrow 31.7$ (C-4), 1.72 (sx, 2$\mathrm{H}) \leftrightarrow 33.3(\mathrm{C}-2), 3.38\left(\mathrm{dd}, 1-\mathrm{H}^{\mathrm{B}}\right) \leftrightarrow 68.6(\mathrm{C}-1), 3.52\left(\mathrm{dd}, 1-\mathrm{H}^{\mathrm{A}}\right) \leftrightarrow 68.6(\mathrm{C}-1)$

Specific rotation $(S, S)-29[a]_{D}^{20}=-4.4^{\circ}\left(\mathrm{c}=1.0\right.$ in $\left.\mathrm{CHCl}_{3}\right)$.

Specific rotation $(R, R)-29[a]_{D}^{20}=+4.8^{\circ}\left(\mathrm{c}=1.0\right.$ in $\left.\mathrm{CHCl}_{3}\right)$.

HRMS (ESI) m/z: [M+NH 4$]^{+}$Calcd for $\mathrm{C}_{8} \mathrm{H}_{22} \mathrm{ON}$ 148.1696; Found 148.1695

IR (KBr): $\tilde{v}=3330,3055,2960,2915,2875,1680,1630,1460,1380,1265,1205,1035,985$, $745,705 \mathrm{~cm}^{-1}$

$(2 R, 4 R)-2,4-D i m e t h y l h e x a n a l ~[(R, R)-12]$

and

$(2 S, 4 S)-2,4-D i m e t h y l h e x a n a l[(S, S)-12]$

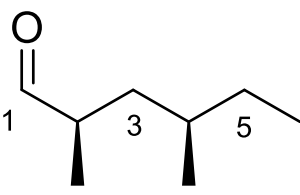

$(R, R)-12$<smiles>CC[C@H](C)C[C@@H](C)C=O</smiles>

$(S, S)-12$

Method A: A solution of the alcohol $(R, R)-29(84 \mathrm{mg}, 0.64 \mathrm{mmol})$ and $N$-methylmorpholine$N$-oxide (151 mg, $1.29 \mathrm{mmol}, 2.0$ eq.) in $\mathrm{CH}_{2} \mathrm{Cl}_{2}(1.0 \mathrm{~mL})$ was treated with molecular sieves 4 $\AA(0.20 \mathrm{~g})$. The mixture stirred $5 \mathrm{~min}$ at $25^{\circ} \mathrm{C}$. Then it was added $\mathrm{Pr}_{4} \mathrm{NRuO}_{4}(11.5 \mathrm{mg}$, $32.0 \mu \mathrm{mol}, 5 \mathrm{~mol}-\%$ ) and held this temperature for $1 \mathrm{~h}$. The mixture was filtered over a pad of Celite ${ }^{\circledR}$. The pad was rinsed with $\mathrm{Et}_{2} \mathrm{O}(20 \mathrm{~mL})$. The solvent was removed under reduced

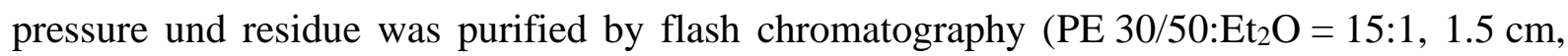


$15 \mathrm{~cm}, 5 \mathrm{~mL}$ ). The product $(R, R)-\mathbf{1 2}\left[65 \mathrm{mg}, 77 \%, 0.50 \mathrm{mmol}, d r=98: 2\right.$ (Lit. $\left.\left.^{10}: 98 \%\right)\right]$ was obtained as a colorless liquid.

The enantiomer $(S, S)-\mathbf{1 2}$ was obtained analogously in $76 \%$ yield.

Method B: A solution of $(\mathrm{COCl})_{2}(210 \mu \mathrm{L}, 2.42 \mathrm{mmol}, 1.1$ eq. $)$ in THF (6.2 mL) was treated with DMSO $(180 \mu \mathrm{L}, 2.48 \mathrm{mmol}, 1.1$ eq. $)$ at $-78^{\circ} \mathrm{C}$. The mixture was stirred for $3 \mathrm{~min}$ at $0^{\circ} \mathrm{C}$ and cooled again to $-78^{\circ} \mathrm{C}$. A solution of the alcohol $(R, R)-29(300 \mathrm{mg}, 2.29 \mathrm{mmol})$ in $\mathrm{THF}$ $(3.3 \mathrm{~mL})$ was added. The mixture was warmed to $0^{\circ} \mathrm{C}$ and held this temperature for $15 \mathrm{~min}$. Then $\mathrm{NEt}_{3}(1.60 \mathrm{~mL}, 1.17 \mathrm{~g}, 11.5 \mathrm{mmol}, 5.0$ eq.) was added and the mixture was warmed to $25^{\circ} \mathrm{C}$. The mixture was diluted with sat. aq. $\mathrm{NH}_{4} \mathrm{Cl}$ solution $(5 \mathrm{~mL})$. The layers were separated und the aq. layer was extracted with $\mathrm{Et}_{2} \mathrm{O}$ :pentane $(4: 1,3 \times 40 \mathrm{~mL})$. The combined organic layers were tried over $\mathrm{Na}_{2} \mathrm{SO}_{4}$ and the solvent was removed under reduced pressure. The residue was purified by flash chromatography (PE 30/50:Et ${ }_{2} \mathrm{O}=15: 1,2.0 \mathrm{~cm}, 15 \mathrm{~cm}, 20 \mathrm{~mL}$ ). The product $(R, R)-\mathbf{1 2}(222 \mathrm{mg}, 76 \%, 1.74 \mathrm{mmol})$ was obtained as a colorless liquid.

The enantiomer $(S, S)-\mathbf{1 2}$ was obtained analogously in $75 \%$ yield.

$\boldsymbol{R}_{\mathbf{f}}\left(\mathrm{PE} 30 / 50: \mathrm{Et}_{2} \mathrm{O}=15: 1\right)=0.52$

${ }^{1} \mathbf{H}$ NMR $\left(300.13 \mathrm{MHz}, \mathrm{CDCl}_{3} / \mathrm{CHCl}_{3}\right.$, sample contained 6 weight- $\%$ of THF and unknown impurity $\mathrm{d}$ at 2.01, DsBrAp23-30090, 23.4.2015): $\delta=0.82-0.91\left(\mathrm{~m}, 3 \mathrm{H}, 4-\mathrm{CH}_{3}\right), 0.82-0.91(\mathrm{~m}$, $\left.3 \mathrm{H}, 6-\mathrm{H}_{3}\right), 1.08\left(\mathrm{~d}, J_{2-\mathrm{CH}_{3}, 2}=7.0 \mathrm{~Hz}, 3 \mathrm{H}, 2-\mathrm{CH}_{3}\right), 1.10-1.16\left(\mathrm{~m}, 1 \mathrm{H}, 5-\mathrm{H}^{\mathrm{A}}\right), 1.10-1.16(\mathrm{~m}, 1 \mathrm{H}$, 3- $\left.\mathrm{H}^{\mathrm{A}}\right), 1.28-1.36(\mathrm{~m}, 1 \mathrm{H}, 4-\mathrm{H}), 1.28-1.36\left(\mathrm{~m}, 1 \mathrm{H}, 5-\mathrm{H}^{\mathrm{B}}\right), 1.38-1.47\left(\mathrm{~m}, 1 \mathrm{H}, 3-\mathrm{H}^{\mathrm{B}}\right), 1.72(\mathrm{~m}$, 4-H), $2.45\left(\mathrm{~m}_{\mathrm{c}}, 1 \mathrm{H}, 2-\mathrm{H}\right), 9.58\left(\mathrm{~d}, J_{1,2}=2.4 \mathrm{~Hz}, 1 \mathrm{H}, 1-\mathrm{H}\right)$.

\section{(E)-N-Cyclohexylpropan-1-imine (SI-2)}

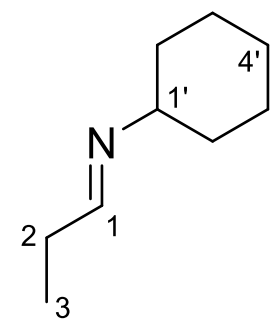

SI-2

To stirred neat cyclohexylamine $(16.0 \mathrm{~mL}, 13.8 \mathrm{~g}, 140 \mathrm{mmol}, 1.0$ eq. $)$ was added at $0{ }^{\circ} \mathrm{C}$ propionaldehyde $(10.0 \mathrm{~mL}, 8.1 \mathrm{~g}, 140 \mathrm{mmol})$ tropwise. After complete addition, stirring was 
continued at $0^{\circ} \mathrm{C}$ for 40 minutes. Then $\mathrm{KOH}$ (about 10 pellets) was added and the mixture was allowed to stand until two clear phases formed. The layers were separated and $\mathrm{KOH}$ (5-6 pellets) was added to the organic phase, which was then allowed to stand in a refrigerator overnight. The solution was decanted, $\mathrm{KOH}$ (5-6 pellets) were added and the material was distilled under reduced pressure (bp.25mbar: $67-70^{\circ} \mathrm{C}$ ). The product SI-2 [17.8 g, 92\%, $128 \mathrm{mmol}$ (Lit. $\left.\left.{ }^{13}: 87 \%\right)\right]$ was obtained as colorless oil.

${ }^{1} \mathbf{H}$ NMR (300.13 MHz, $\mathrm{CDCl}_{3} / \mathrm{CHCl}_{3}$, DsBrJa29-30060, 29.1.2018): $\delta=1.09$ (t, $J_{3,2}=7.5 \mathrm{~Hz}$, $\left.3 \mathrm{H}, 3-\mathrm{H}_{3}\right), 1.17-1.40\left(\mathrm{~m}, 3 \mathrm{H}, 3^{\prime}-\mathrm{H}^{\mathrm{B}}, 5^{\prime}-\mathrm{H}^{\mathrm{B}}, 4^{\prime}-\mathrm{H}^{\mathrm{B}}\right), 1.42-1.56\left(\mathrm{~m}, 2 \mathrm{H}, 3^{\prime}-\mathrm{H}^{\mathrm{A}}, 5^{\prime}-\mathrm{H}^{\mathrm{A}}\right), 1.60-1.70$ $\left(\mathrm{m}, 3 \mathrm{H}, 2^{\prime}-\mathrm{H}^{\mathrm{B}}, 6^{\prime}-\mathrm{H}^{\mathrm{B}}, 4^{\prime}-\mathrm{H}^{\mathrm{A}}\right), 1.75-1.84\left(\mathrm{~m}, 2 \mathrm{H}, 2^{\prime}-\mathrm{H}^{\mathrm{A}}, 6^{\prime}-\mathrm{H}^{\mathrm{A}}\right), 2.45\left(\mathrm{dq}, J_{2,3}=7.6 \mathrm{~Hz}\right.$, $\left.J_{2,1}=4.9 \mathrm{~Hz}, 2 \mathrm{H}, 2-\mathrm{H}_{2}\right), 2.93\left(\mathrm{tt}, J_{1^{\prime}, 2^{\mathrm{A}} / 6^{\mathrm{A}}}=10.8 \mathrm{~Hz}, J_{1^{\prime}, 2^{1 \mathrm{~B}} / 6^{\mathrm{B}}}=4.1 \mathrm{~Hz}, 1 \mathrm{H}, 1^{\prime}-\mathrm{H}\right), 7.68(\mathrm{t}$, $\left.J_{1,2}=4.9 \mathrm{~Hz}, 1 \mathrm{H}, 1-\mathrm{H}\right)$.

\section{(E)-N-Cyclohexyl-2-(triethylsilyl)propan-1-imine (30)}

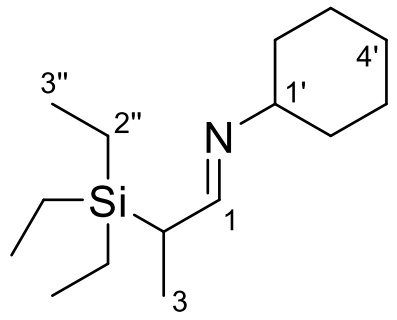

30

A solution of diisopropylamine $(2.10 \mathrm{~mL}, 1.51 \mathrm{~g}, 15.1 \mathrm{mmol}, 1.1$ eq.) in THF (18 mL) was treated with $n \mathrm{BuLi}\left(2.45 \mathrm{M}\right.$ in hexane, $6.13 \mathrm{~mL}, 15.1 \mathrm{mmol}, 1.1$ eq.) at $-78{ }^{\circ} \mathrm{C}$. The solution was stirred at $-78{ }^{\circ} \mathrm{C}$ for 30 minutes and then at $0^{\circ} \mathrm{C}$ for 15 minutes. The imine SI-2 $(2.00 \mathrm{~g}$, $14.4 \mathrm{mmol})$ in THF $(3.6 \mathrm{~mL})$ was added dropwise. After 35 minutes at $-78^{\circ} \mathrm{C} \mathrm{TESCl}(2.50 \mathrm{~mL}$, $2.25 \mathrm{~g}, 15.1 \mathrm{mmol}, 1.1 \mathrm{eq}$.) was added. The reaction mixture was allowed to warm to $0^{\circ} \mathrm{C}$ over a period of $3 \mathrm{~h}$ and stirred at that temperature for 15 minutes. Then water $(100 \mathrm{~mL})$ was added together with $\mathrm{Et}_{2} \mathrm{O}(50 \mathrm{~mL})$. The phases were separated and the aq. layer was extracted with $\mathrm{Et}_{2} \mathrm{O}(3 \times 50 \mathrm{~mL})$. The combined organic layers were dried over $\mathrm{K}_{2} \mathrm{CO}_{3}$, filtered and concentrated under reduced pressure. Distillation (bp.0.6mbar: $120-125^{\circ} \mathrm{C}$ ) afforded 30 [2.51 g, $69 \%, 9.94$ mmol (Lit. $\left.\left.{ }^{13}: 88 \%\right)\right]$ as a pale yellow oil.

\footnotetext{
${ }^{13}$ F. Glaus, K.-H. Altmann, Angew. Chem. Int. Ed. 2015, 54, 1937 -1940.
} 
${ }^{1} \mathbf{H}$ NMR $\left(300.13 \mathrm{MHz}, \mathrm{CDCl}_{3} / \mathrm{CHCl}_{3}\right.$, sample contained unknown impurity s at 2.38, DsBrJa30-30040, 30.1.2019): 0.59 (q, $\left.J_{2^{\prime \prime}, 3^{\prime \prime}}=8.0 \mathrm{~Hz}, 6 \mathrm{H}, 3 \times 2 "-\mathrm{H}_{2}\right), 0.95\left(\mathrm{t}, J_{3^{\prime \prime}, 2^{\prime \prime}}=8.0 \mathrm{~Hz}, 9 \mathrm{H}\right.$, $\left.3 \times 3^{\prime \prime}-\mathrm{H}_{3}\right), 1.16\left(\mathrm{~d}, J_{3,2}=7.2 \mathrm{~Hz}, 3 \mathrm{H}, 3-\mathrm{H}_{3}\right), 1.17-1.37\left(\mathrm{~m}, 3 \mathrm{H}, 3^{\prime}-\mathrm{H}^{\mathrm{B}}, 5^{\prime}-\mathrm{H}^{\mathrm{B}}, 4^{\prime}-\mathrm{H}^{\mathrm{B}}\right), 1.40-1.55$ $\left(\mathrm{m}, 2 \mathrm{H}, 3^{\prime}-\mathrm{H}^{\mathrm{A}}, 5^{\prime}-\mathrm{H}^{\mathrm{A}}\right), 1.56-1.67\left(\mathrm{~m}, 3 \mathrm{H}, 2^{\prime}-\mathrm{H}^{\mathrm{B}}, 6^{\prime}-\mathrm{H}^{\mathrm{B}}, 4^{\prime}-\mathrm{H}^{\mathrm{A}}\right), 1.72-1.82\left(\mathrm{~m}, 2 \mathrm{H}, 2^{\prime}-\mathrm{H}^{\mathrm{A}}, 6^{\prime}-\mathrm{H}^{\mathrm{A}}\right)$, $2.03\left(\mathrm{q}, J_{2,3}=J_{2,1}=6.9 \mathrm{~Hz}, 1 \mathrm{H}, 2-\mathrm{H}\right), 2.87\left(\mathrm{tt}, J_{1^{\prime}, 2^{\mathrm{A}} / 6^{\mathrm{A}}}=10.5 \mathrm{~Hz}, J_{1^{\prime}, 2^{\mathrm{B}} / 6^{\mathrm{B}}}=4.2 \mathrm{~Hz}, 1 \mathrm{H}, 1^{\prime}-\mathrm{H}\right)$, $7.65\left(\mathrm{dd}, J_{1,2}=6.7 \mathrm{~Hz},{ }^{4} J_{1,1^{\prime}}=0.6 \mathrm{~Hz}, 1 \mathrm{H}, 1-\mathrm{H}\right)$.

$(4 R, 6 R, E)-2,4,6-T r i m e t h y l o c t-2-e n a l ~[(R, R)-10]$

and

$(4 S, 6 S, E)-2,4,6-$ Trimethyloct-2-enal $[(S, S)-10]$<smiles>CC[C@H](C)C[C@@H](C)/C=C(\C)C=O</smiles>

$(R, R)-10$<smiles>CC[C@H](C)C[C@@H](C)/C=C(\C)C=O</smiles>

$(S, S)-10$

A solution of the imine $30(214 \mathrm{mg}, 0.84 \mathrm{mmol}, 1.4 \mathrm{eq}$.$) in THF (1.1 \mathrm{~mL})$ was treated with $s$ BuLi (1.4 M in hexane, $0.55 \mathrm{~mL}, 0.78 \mathrm{mmol}, 1.3$ eq.) at $-78^{\circ} \mathrm{C}$. The mixture was stirred at $-78^{\circ} \mathrm{C}$ for $30 \mathrm{~min}$. Then the aldehyde $(R, R)-12(70 \mathrm{mg}, 0.60 \mathrm{mmol})$ in THF $(0.6 \mathrm{~mL})$ was added, the mixture was warmed to $-20^{\circ} \mathrm{C}$ and stirred at this temperature for $2.5 \mathrm{~h}$. It was quenched with $\mathrm{H}_{2} \mathrm{O}(3 \mathrm{~mL})$, the layers were separated and the organic layer was extracted with $\mathrm{Et}_{2} \mathrm{O}$ $(3 \times 10 \mathrm{~mL})$. The combined organic layers were washed with brine $(5 \mathrm{~mL})$ and tried over $\mathrm{MgSO}_{4}$. The combined organic layers were concentrated under reduced pressure. The obtained imine was dissolved in THF $(2.8 \mathrm{~mL})$. The mixture was treated with $\mathrm{CF}_{3} \mathrm{CO}_{2} \mathrm{H}(65 \mu \mathrm{L}, 0.66$ mmol, 1.1 eq.) at $0^{\circ} \mathrm{C}$ and stirred at this temperature for $1 \mathrm{~h}$. Then $\mathrm{H}_{2} \mathrm{O}(1.1 \mathrm{~mL})$ was added and the mixture stirred for $12 \mathrm{~h}$ at $0^{\circ} \mathrm{C}$. The mixture was quenched with sat. aq. $\mathrm{NaHCO}_{3}(3 \mathrm{~mL})$, the layers were separated and the aq. layer was extracted with $\mathrm{Et}_{2} \mathrm{O}(3 \times 10 \mathrm{~mL})$. The combined organic layers were washed with brine $(10 \mathrm{~mL})$ und tried over $\mathrm{MgSO}_{4}$. The combined organic layers were concentrated under reduced pressure. Purification by flash chromatography (PE $\left.30 / 50: \mathrm{Et}_{2} \mathrm{O}=35: 1,2.0 \mathrm{~cm}, 25 \mathrm{~cm}, 15 \mathrm{~mL}\right)$ afforded $(R, R)-10(79 \mathrm{mg}, 86 \%, 0.52 \mathrm{mmol})$ as a colorless liquid.

The enantiomer $(S, S)-\mathbf{1 0}$ was obtained analogously in $86 \%$ yield. 
Christian Drescher, Morris Keller, Olivier Potterat, Matthias Hamburger and Reinhard Brückner, Structure-Elucidating Total Synthesis of the (Polyenoyl)tetramic Acid Militarinone C

$\mathbf{R}_{\mathbf{f}}\left(\mathrm{PE} 30 / 50: \mathrm{Et}_{2} \mathrm{O}=35: 1\right)=0.31$

${ }^{1} \mathbf{H}$ NMR $\left(500.10 \mathrm{MHz}, \mathrm{CDCl}_{3} / \mathrm{CHCl}_{3}\right.$, sample contained $\mathrm{H}_{2} \mathrm{O}$, DsBrJa20-5060, 3.2.2017): $\delta=$ $0.85\left(\mathrm{~d}, J_{6-\mathrm{CH}_{3}, 6}=6.2 \mathrm{~Hz}, 3 \mathrm{H}, 6-\mathrm{CH}_{3}\right), 0.86\left(\mathrm{t}, J_{8,7}=7.3 \mathrm{~Hz}, 3 \mathrm{H}, 8-\mathrm{H}_{3}\right), 1.05\left(\mathrm{~d}, J_{4-\mathrm{CH}_{3}, 4}=6.6 \mathrm{~Hz}\right.$, $\left.3 \mathrm{H}, 4-\mathrm{CH}_{3}\right), 1.11-1.19\left(\mathrm{~m}, 1 \mathrm{H}, 7-\mathrm{H}^{\mathrm{A}}\right), 1.20-1.26\left(\mathrm{~m}, 1 \mathrm{H}, 5-\mathrm{H}^{\mathrm{A}}\right), 1.20-1.26(\mathrm{~m}, 1 \mathrm{H}, 6-\mathrm{H}), 1.27-$ $1.34\left(\mathrm{~m}, 1 \mathrm{H}, 7-\mathrm{H}^{\mathrm{B}}\right), 1.38-1.43\left(\mathrm{~m}, 1 \mathrm{H}, 5-\mathrm{H}^{\mathrm{B}}\right), 1.76\left(\mathrm{~d},{ }^{4} \mathrm{~J}_{2-\mathrm{CH}_{3}, 3}=1.4 \mathrm{~Hz}, 3 \mathrm{H}, 2-\mathrm{CH}_{3}\right), 2.81\left(\mathrm{~m}_{\mathrm{c}}\right.$, $1 \mathrm{H}, 4-\mathrm{H}), 6.22\left(\mathrm{dq}, J_{3,4}=10.0 \mathrm{~Hz},{ }^{4} J_{3,2-\mathrm{CH}_{3}}=1.4 \mathrm{~Hz}, 1 \mathrm{H}, 3-\mathrm{H}\right), 9.39$ (s, 1H, 1-H).

${ }^{13} \mathrm{C}$ NMR (125.75 MHz, $\mathrm{CDCl}_{3} / \mathrm{CHCl}_{3}$, DsBrJa20-5064, 3.2.2017): $\delta=9.4\left(2-\mathrm{CH}_{3}\right), 11.3(\mathrm{C}-$ 8), $19.1\left(6-\mathrm{CH}_{3}\right), 20.5\left(4-\mathrm{CH}_{3}\right), 30.1$ (C-7), 31.3 (C-4), 32.5 (C-6), 44.1 (C-5), 137.9 (C-2), 160.9 (C-3), 195.7 (C-1).

edHSQC [,,short-range H,C-COSY“, $500.10 \mathrm{MHz} / 125.75 \mathrm{MHz}, \mathrm{CDCl}_{3} ; \delta\left({ }^{1} \mathrm{H}\right) \leftrightarrow \delta\left({ }^{13} \mathrm{C}\right): 0.85$ $\left(\mathrm{d}, 6-\mathrm{CH}_{3}\right) \leftrightarrow 19.1\left(6-\mathrm{CH}_{3}\right), 0.86\left(\mathrm{t}, 8-\mathrm{H}_{3}\right) \leftrightarrow 11.3(\mathrm{C}-8), 1.05\left(\mathrm{~d}, 4-\mathrm{CH}_{3}\right) \leftrightarrow 20.5\left(4-\mathrm{CH}_{3}\right)$, 1.11-1.19 (m, 7- $\left.\mathrm{H}^{\mathrm{A}}\right) \leftrightarrow 30.1(\mathrm{C}-7), 1.20-1.26\left(\mathrm{~m}, 5-\mathrm{H}^{\mathrm{A}}\right) \leftrightarrow 44.1(\mathrm{C}-5), 1.20-1.26(\mathrm{~m}, 6-\mathrm{H}) \leftrightarrow$ $32.5(\mathrm{C}-6), 1.27-1.34\left(\mathrm{~m}, 7-\mathrm{H}^{\mathrm{B}}\right) \leftrightarrow 30.1(\mathrm{C}-7), 1.38-1.43\left(\mathrm{~m}, 5-\mathrm{H}^{\mathrm{B}}\right) \leftrightarrow 44.1(\mathrm{C}-5), 1.76(\mathrm{~d}, 2-$ $\left.\mathrm{CH}_{3}\right) \leftrightarrow 9.4\left(2-\mathrm{CH}_{3}\right), 2.81\left(\mathrm{~m}_{\mathrm{c}}, 4-\mathrm{H}\right) \leftrightarrow 31.3(\mathrm{C}-4), 6.22(\mathrm{dq}, 3-\mathrm{H}) \leftrightarrow 160.9(\mathrm{C}-3), 9.39$ (s, $1-$ $\mathrm{H}) \leftrightarrow 195.7(\mathrm{C}-1)$.

Specific rotation $(S, S)-\mathbf{1 0}[a]_{D}^{20}=42.0^{\circ}\left(\mathrm{c}=1.0\right.$ in $\left.\mathrm{CHCl}_{3}\right)$.

Specific rotation $(R, R)-\mathbf{1 0}[a]_{D}^{20}=-41.8^{\circ}\left(\mathrm{c}=1.0\right.$ in $\left.\mathrm{CHCl}_{3}\right)$, Lit. $^{14}:[a]_{D}^{20}=-43.0^{\circ}(\mathrm{c}=0.75$ in $\mathrm{CHCl}_{3}$ ).

HRMS (ESI) m/z: $\left[\mathrm{M}+\mathrm{NH}_{4}\right]^{+}$Calcd for $\mathrm{C}_{11} \mathrm{H}_{24} \mathrm{ON} 186.1852$; Found 186.1853

IR (KBr): $\tilde{v}=2965,2930,2875,2875,2855,2815,2765,2705,1690,1645,1460,1405,1380$, 1360, 1315, 1275, 1260, 1245, 1200, 1152, 1130, 1050, 1015, 970, 875, 825, 805, $675 \mathrm{~cm}^{-1}$.

\footnotetext{
${ }^{14}$ H. J. Jessen, A. Schumacher, T. Shaw, A. Pfaltz, K. Gademann, Angew. Chem. Int. Ed. 2011, 50, 4222-4226.
} 


\section{Dimethyl (2-Oxopropyl)phosphonate (SI-3)}<smiles>COP(=O)(CC(C)=O)OC</smiles>

\section{SI-3}

A solution of chloroacetone (5.00 mL, $5.75 \mathrm{~g}, 54.0 \mathrm{mmol})$ and $\mathrm{KI}(9.00 \mathrm{~g}, 54.0 \mathrm{mmol}, 1.0 \mathrm{eq}$. in acetone $(11 \mathrm{~mL})$ and acetonitrile $(20 \mathrm{~mL})$ was stirred at room temperature for $2 \mathrm{~h}$ under light exclusion. Then trimethylphosphite $(6.45 \mathrm{ml}, 6.71 \mathrm{~g}, 54.0 \mathrm{mmol}, 1.0 \mathrm{eq}$.) was added, and the solution was stirred at $50^{\circ} \mathrm{C}$ for $24 \mathrm{~h}$. The mixture was cooled to $25^{\circ} \mathrm{C}$ and the precipitate was filtered off. After evaporation of the solvent under reduced pressure the crude product was distilled (bp.2mabr: 88-90 ${ }^{\circ}$ ). The product SI-3 [7.97 g, 77\%, $41.6 \mathrm{mmol}$ (Lit. ${ }^{15}$ : 82\%) was obtained as a colorless liquid.

${ }^{1} \mathbf{H}$ NMR (300.13 MHz, $\left.\mathrm{CDCl}_{3} / \mathrm{CHCl}_{3}, \mathrm{DsBrDe} 22-30250,22.12 .2016\right): \delta=2.33\left(\mathrm{~s}, 3 \mathrm{H}, 3-\mathrm{H}_{3}\right)$, $3.10\left(\mathrm{~d}, J_{1, \mathrm{P}}=22.8 \mathrm{~Hz}, 2 \mathrm{H}, 1-\mathrm{H}_{2}\right), 3.78$ [s, 3H, P(O)-OMe ${ }^{\mathrm{A}}$, 3.81 [s, 3H, $\left.\mathrm{P}(\mathrm{O})-\mathrm{OMe}^{\mathrm{B}}\right]$.

\section{Dimethyl (1-Diazo-2-oxopropyl)phosphonate (SI-4)}<smiles>COP(=O)(OC)C(=N[Cl+])C(C)=O</smiles>

At $0^{\circ} \mathrm{C} \mathrm{SI-3}(10.0 \mathrm{~g}, 60.0 \mathrm{mmol})$ was dissolved in toluene $(120 \mathrm{~mL})$ and $\mathrm{NaH}(60 \mathrm{wt}-\%, 4.80 \mathrm{~g}$, $72.0 \mathrm{mmol}, 2.0$ eq.) was added portion wise. After $1 \mathrm{~h}$ at the same temperature, a solution of $p$ ABSA (13.0 g, $54.0 \mathrm{mmol}, 0.9$ eq.) in THF (40 mL) was added dropwise. The reaction mixture was stirred at room temperature for $24 \mathrm{~h}$. PE 30/50 (50 mL) was added, then the precipitate was filtered off, and the filter cake was washed with $\mathrm{Et}_{2} \mathrm{O}(3 \times 50 \mathrm{~mL})$. The filtrate was evaporated and the residue was purified by flash chromatography $(\mathrm{PE} \mathrm{30/50:AcOEt}=1: 1,6 \mathrm{~cm}, 15 \mathrm{~cm}, 50$ $\mathrm{mL}$ ). The product SI-4 [6.24 g, 60\%, $32.3 \mathrm{mmol}$ (Lit. ${ }^{15}$ : 92\%)] was obtained as a yellow liquid.

\footnotetext{
15 T. Du, F. Du, Y. Ning, Y. Peng, Org.Lett. 2015, 17, 1308-1311.
} 


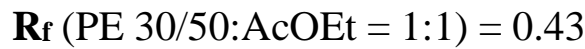

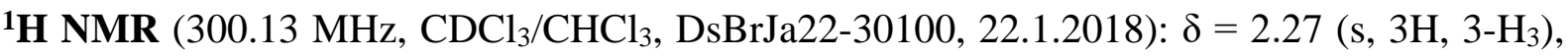
$3.82\left[\mathrm{~s}, 3 \mathrm{H}, \mathrm{P}(\mathrm{O})-\mathrm{OMe}^{\mathrm{A}}\right], 3.86\left[\mathrm{~s}, 3 \mathrm{H}, \mathrm{P}(\mathrm{O})-\mathrm{OMe}^{\mathrm{B}}\right]$.

\section{Dimethyl (Diazomethyl)phosphonate (31)}<smiles>CO[P](=O)/C=N\[N+]=O</smiles>

31

A solution of SI-4 $(3.47 \mathrm{~g}, 18.3 \mathrm{mmol})$ in $\mathrm{MeOH}(26 \mathrm{~mL})$ was treated with $\mathrm{K}_{2} \mathrm{CO}_{3}(0.75 \mathrm{~g}$, $5.3 \mathrm{mmol}, 0.3 \mathrm{eq})$ at $25^{\circ} \mathrm{C}$. The mixture held this temperature for $15 \mathrm{~min}$ and filtered off. The filter cake was rinsed with $\mathrm{CH}_{2} \mathrm{Cl}_{2}(20 \mathrm{~mL})$. The solvent was removed under reduced pressure and the residue was purified by flash chromatography $(\mathrm{PE} \mathrm{30/50:AcOEt}=1: 1,3.5 \mathrm{~cm}, 15 \mathrm{~cm}$, $30 \mathrm{~mL}$ ). The product 31 [1.34 g, 49\%, $9.12 \mathrm{mmol}$ (Lit. ${ }^{16}: 85 \%$ )] was obtained as a yellow oil.

$\mathbf{R}_{\mathbf{f}}(\mathrm{PE} 30 / 50: \mathrm{AcOEt}=1: 1)=0.49$

${ }^{1} \mathbf{H}$ NMR $\left(300.13 \mathrm{MHz}, \mathrm{CDCl}_{3} / \mathrm{CHCl}_{3}, \mathrm{DsBrAu07-30600,7.8.2018):} \delta=3.75[\mathrm{~s}, 3 \mathrm{H}, \mathrm{P}(\mathrm{O})-\right.$ $\mathrm{OMe}^{\mathrm{A}}$ ], $3.76\left(\mathrm{~d}, J_{1, \mathrm{P}}=11.1 \mathrm{~Hz}, 1 \mathrm{H}, 1-\mathrm{H}\right), 3.80\left[\mathrm{~s}, 3 \mathrm{H}, \mathrm{P}(\mathrm{O})-\mathrm{OMe}^{\mathrm{B}}\right]$. 
and

$(5 S, 7 S, E)-3,5,7-T r i m e t h y l n o n-3-e n-1-y n e ~[(S, S)-32]$

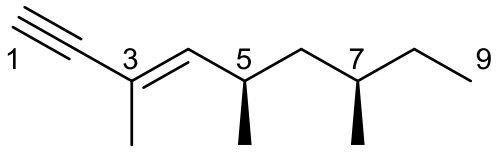

$(R, R)-32$

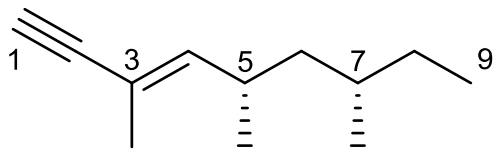

$(S, S)-32$

A solution of 31 (32.0 mg, $180 \mu \mathrm{mol}, 1.5$ eq.) in THF (1.1 mL) was treated with $\mathrm{KO} t \mathrm{Bu}(1 \mathrm{M}$ in THF, $180 \mu \mathrm{L}, 180 \mu \mathrm{mol}, 1.5$ eq.) at $-78^{\circ} \mathrm{C}$. The mixture was stirred at $-78{ }^{\circ} \mathrm{C}$ for $15 \mathrm{~min}$. A solution of the aldehyde $(R, R)-\mathbf{1 0}(20.0 \mathrm{mg}, 120 \mu \mathrm{mol})$ in THF $(0.3 \mathrm{~mL})$ was added. The mixture was stirred first $5 \mathrm{~min}$ at $-78^{\circ} \mathrm{C}$ and then for $1.5 \mathrm{~h}$ at $-30^{\circ} \mathrm{C}$. The mixture was diluted with sat. aq. $\mathrm{NH}_{4} \mathrm{Cl}$ solution $(3 \mathrm{~mL})$, stirred for $15 \mathrm{~min}$ at $-30^{\circ} \mathrm{C}$ and warmed to $25^{\circ} \mathrm{C}$. The phases were separated and the aq. layer was extracted with $\mathrm{Et}_{2} \mathrm{O}(3 \times 10 \mathrm{~mL})$. The combined organic layers were dried over $\mathrm{Na}_{2} \mathrm{SO}_{4}$ and concentrated under reduced pressure (250 mbar for $10 \mathrm{~min}$ ). The residue was purified by flash chromatography (pentane, $1.0 \mathrm{~cm}, 10 \mathrm{~cm}, 3 \mathrm{~mL}$ ). The alkyne $(R, R)-32(16.2 \mathrm{mg}, 83 \%, 110 \mu \mathrm{mol})$ was obtained as a colorless liquid.

The enantiomer $(S, S)$-32 was obtained analogously in $82 \%$ yield.

$\mathbf{R}_{\mathbf{f}}($ pentane $)=0.75$

${ }^{1} \mathrm{H}$ NMR $\left(500.10 \mathrm{MHz}, \mathrm{CDCl}_{3} / \mathrm{CHCl}_{3}\right.$, sample contained $\mathrm{H}_{2} \mathrm{O}$ and 1 weight-\% of $\mathrm{Et}_{2} \mathrm{O}$, DsBrJa20-5050, 3.2.2017): 0.82 (d, $\left.J_{7-\mathrm{CH}_{3}, 7}=6.4 \mathrm{~Hz}, 3 \mathrm{H}, 7-\mathrm{CH}_{3}\right), 0.85$ (t, $J_{9,8}=7.3 \mathrm{~Hz}, 3 \mathrm{H}, 9$ $\left.\mathrm{H}_{3}\right), 0.94\left(\mathrm{~d}, J_{5-\mathrm{CH}_{3}, 5}=6.7 \mathrm{~Hz}, 3 \mathrm{H}, 5-\mathrm{CH}_{3}\right), 1.03-1.17\left(\mathrm{~m}, 1 \mathrm{H}, 6-\mathrm{H}^{\mathrm{A}}\right), 1.03-1.17\left(\mathrm{~m}, 1 \mathrm{H}, 8-\mathrm{H}^{\mathrm{A}}\right)$, 1.23-1.33 (m, $\left.1 \mathrm{H}, 6-\mathrm{H}^{\mathrm{B}}\right), 1.23-1.33(\mathrm{~m}, 1 \mathrm{H}, 7-\mathrm{H}), 1.23-1.33\left(\mathrm{~m}, 1 \mathrm{H}, 8-\mathrm{H}^{\mathrm{B}}\right), 1.80\left(\mathrm{~d},{ }^{4} J_{3-\mathrm{CH}_{3,4}}=\right.$ $\left.1.5 \mathrm{~Hz}, 3 \mathrm{H}, 3-\mathrm{CH}_{3}\right), 2.54\left(\mathrm{~m}_{\mathrm{c}}, 1 \mathrm{H}, 5-\mathrm{H}\right), 2.74(\mathrm{~s}, 1 \mathrm{H}, 1-\mathrm{H}), 5.69\left(\mathrm{dq}, J_{4,5}=9.9 \mathrm{~Hz},{ }^{4} J_{4,3-\mathrm{CH}_{3}}=1.5\right.$ $\mathrm{Hz}, 1 \mathrm{H}, 4-\mathrm{H})$.

${ }^{13} \mathrm{C}$ NMR $\left(125.75 \mathrm{MHz}, \mathrm{CDCl}_{3} / \mathrm{CHCl}_{3}\right.$, sample contained $\mathrm{Et}_{2} \mathrm{O}$ and pentane, DsBrJa20-5054, 3.2.2017): $\delta=11.3(\mathrm{C}-9), 17.2\left(3-\mathrm{CH}_{3}\right), 19.1\left(7-\mathrm{CH}_{3}\right), 21.0\left(5-\mathrm{CH}_{3}\right), 30.2(\mathrm{C}-8), 30.7(\mathrm{C}-5)$, 32.3 (C-7), 44.5 (C-6), 73.3 (C-1), 87.2 (C-2), 115.2 (C-3), 146.4 (C-4).

edHSQC [,,short-range H,C-COSY“, $500.10 \mathrm{MHz} / 125.75 \mathrm{MHz}, \mathrm{CDCl}_{3} ; \delta\left({ }^{1} \mathrm{H}\right) \leftrightarrow \delta\left({ }^{13} \mathrm{C}\right): 0.82$ $\left(\mathrm{d}, 7-\mathrm{CH}_{3}\right) \leftrightarrow 19.1\left(7-\mathrm{CH}_{3}\right), 0.85\left(\mathrm{t}, 9-\mathrm{H}_{3}\right) \leftrightarrow 11.3(\mathrm{C}-9), 0.94\left(\mathrm{~d}, 5-\mathrm{CH}_{3}\right) \leftrightarrow 21.0\left(5-\mathrm{CH}_{3}\right)$ 
1.03-1.17 (m, 6- $\left.\mathrm{H}^{\mathrm{A}}\right) \leftrightarrow 44.5(\mathrm{C}-6), 1.03-1.17\left(\mathrm{~m}, 8-\mathrm{H}^{\mathrm{A}}\right) \leftrightarrow 30.2(\mathrm{C}-8), 1.23-1.33\left(\mathrm{~m}, 6-\mathrm{H}^{\mathrm{B}}\right) \leftrightarrow$ 44.5 (C-6), 1.23-1.33 (m, 7-H) $32.3(\mathrm{C}-7), 1.23-1.33\left(\mathrm{~m}, 8-\mathrm{H}^{\mathrm{B}}\right) \leftrightarrow 30.2(\mathrm{C}-8), 1.80(\mathrm{~d}, 3-$ $\left.\mathrm{CH}_{3}\right) \leftrightarrow 17.2\left(3-\mathrm{CH}_{3}\right), 2.54\left(\mathrm{~m}_{\mathrm{c}}, 5-\mathrm{H}\right) \leftrightarrow 30.7(\mathrm{C}-5), 2.74(\mathrm{~s}, 1-\mathrm{H}) \leftrightarrow 73.3(\mathrm{C}-1), 5.69$ (dq, $4-$ $\mathrm{H}) \leftrightarrow 146.4(\mathrm{C}-4)$.

Specific rotation $(S, S)-32[a]_{D}^{20}=61.0^{\circ}\left(\mathrm{c}=1.0\right.$ in $\left.\mathrm{CHCl}_{3}\right)$.

Specific rotation $(R, R)-32[a]_{D}^{20}=-60.5^{\circ}\left(\mathrm{c}=1.0\right.$ in $\left.\mathrm{CHCl}_{3}\right)$.

HRMS (ESI) m/z: [M+H $\left.\mathrm{H}_{3} \mathrm{O}\right]^{+}$Calcd for $\mathrm{C}_{12} \mathrm{H}_{23} \mathrm{O}$ 183.1744; Found 183.1745

IR (KBr): $\tilde{v}=3315,3300,2960,2925,2875,2855,2095,1455,1380,1355,1305,1275,1260$, $1235,1190,1110,1045,1035,995,965,885,875,860,635,600,555,525 \mathrm{~cm}^{-1}$. 


\subsection{Synthesis of all 4 Possible Diastereomeres of the Tetramic Acid Militarinone C (1)}

Methyl (2'S)-2'-\{ $N$-[(1E,2Z,4E,6E,8E,10S,12S)-3-Hydroxy-8,10,12-trimethyltetradeca-

2,4,6,8-tetraenamido]- $N-(1 "$ '',5'"'-dimethoxybenzyl $)\}-3$ '- $\{1$ "'-[(tert-

butyldimethylsilyl)oxy $]$ phenyl $\}$ propanoate $[E-e n o l-(S, S, S)-33]$

in an equilibrium with

Methyl (2'S)-2'- $\{N-[(1 Z, 2 Z, 4 E, 6 E, 8 E, 10 S, 12 S)-3-H y d r o x y-8,10,12$-trimethyltetradeca-

2,4,6,8-tetraenamido]- $N-\left(1{ }^{\prime \prime \prime}, 5\right.$ '"'-dimethoxybenzyl $\left.)\right\}-3$ '- $\{1$ ''-[(tert-

butyldimethylsilyl)oxy]phenyl $\}$ propanoate $[Z-e n o l-(S, S, S)-33]$

in an equilibrium with

Methyl (2' $S)-2 '-\{N-[(1 E, 4 E, 6 E, 8 E, 10 S, 12 S)-3-0 \times 0-8,10,12-t r i m e t h y l t e t r a d e c a-4,6,8-$

trienamido]- $N-(1$ '",5"'-dimethoxybenzyl $)\}-3 '-\{1$ "'-[(tert-

butyldimethylsilyl)oxy]phenyl $\}$ propanoate $[$ E-keto-(S,S,S)-33]

in an equilibrium with

Methyl (2'S)-2'- $\{N$-[(1Z,4E,6E,8E,10S,12S)-3-Oxo-8,10,12-trimethyltetradeca-4,6,8-

trienamido]- $N-(1 " ', 5$ "'-dimethoxybenzyl $)\}-3 '-\{1$ "-[(tert-

butyldimethylsilyl)oxy]phenyl $\}$ propanoate $[Z-k e t o-(S, S, S)-33]$

and

Methyl $\left(2^{\prime} R\right)-2^{\prime}-\{N-[(1 E, 2 Z, 4 E, 6 E, 8 E, 10 R, 12 R)-3-H y d r o x y-8,10,12$-trimethyltetradeca-

2,4,6,8-tetraenamido]- $N-\left(1{ }^{\prime \prime}, 5\right.$ '"'-dimethoxybenzyl $\left.)\right\}-3$ '- $\{1$ "-[(tert-

butyldimethylsilyl)oxy]phenyl $\}$ propanoate $[$ E-enol-(R,R,R)-33]

in an equilibrium with

Methyl (2' $R)-2 '-\{N-[(1 Z, 2 Z, 4 E, 6 E, 8 E, 10 R, 12 R)-3-H y d r o x y-8,10,12$-trimethyltetradeca-

2,4,6,8-tetraenamido]-N-(1"',5'"-dimethoxybenzyl) $\}-3$ '- $\{1$ ''-[(tert-

butyldimethylsilyl)oxy]phenyl $\}$ propanoate $[Z$-enol-(R,R,R)-33]

in an equilibrium with 
Christian Drescher, Morris Keller, Olivier Potterat, Matthias Hamburger and Reinhard Brückner, Structure-Elucidating Total Synthesis of the (Polyenoyl)tetramic Acid Militarinone C

Methyl (2'R)-2'- $\{N-[(1 E, 4 E, 6 E, 8 E, 10 R, 12 R)-3-0 x 0-8,10,12-t r i m e t h y l t e t r a d e c a-4,6,8-$ trienamido]- $N-(1 " ', 5$ '"'-dimethoxybenzyl $)\}-3$ '-\{1'"-[(tertbutyldimethylsilyl)oxy]phenyl $\}$ propanoate $[$ E-keto- $(R, R, R)-33]$ in an equilibrium with

Methyl (2'R)-2'- $\{N-[(1 Z, 4 E, 6 E, 8 E, 10 R, 12 R)-3-0 x 0-8,10,12-t r i m e t h y l t e t r a d e c a-4,6,8-$ trienamido]- $N-(1 " ', 5$ "'-dimethoxybenzyl $)\}-3 '-\{1$ "'-[(tertbutyldimethylsilyl)oxy]phenyl $\}$ propanoate $[Z-k e t o-(R, R, R)-33]$ 
Christian Drescher, Morris Keller, Olivier Potterat, Matthias Hamburger and Reinhard Brückner, Structure-Elucidating Total Synthesis of the (Polyenoyl)tetramic Acid Militarinone C
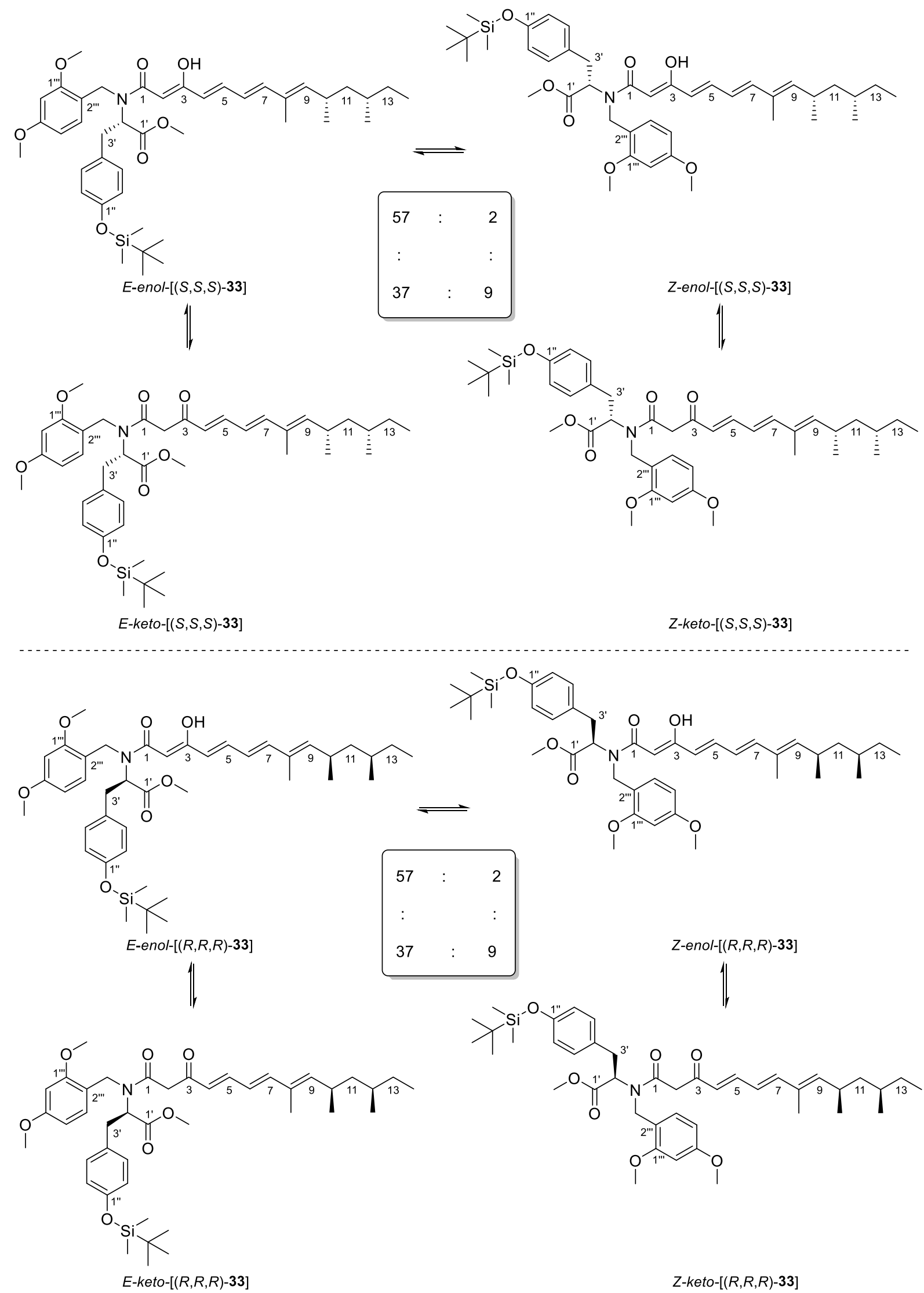

The alkyne $(S, S)-32(45.0 \mathrm{mg}, 246 \mu \mathrm{mol})$ was dissolved in THF $(1.5 \mathrm{~mL})$. It was added $\mathrm{Pd}\left(\mathrm{PPh}_{3}\right)_{2} \mathrm{Cl}_{2}(17.6 \mathrm{mg}, 25 \mu \mathrm{mol}, 10 \mathrm{~mol}-\%)$ and a solution of $\mathrm{Bu}_{3} \mathrm{SnH}(107 \mathrm{mg}, 367 \mu \mathrm{mol}$, 
1.5 eq. $)$ in THF $(1.0 \mathrm{~mL})$. The mixture was stirred at $25^{\circ} \mathrm{C}$ for $30 \mathrm{~min}$. Then a solution of $(S)-8$ (108 mg, $172 \mu \mathrm{mol}, 0.7$ eq.) in THF (1.5 mL) was added. Immediately it was added $\operatorname{Pd}_{2}(\mathrm{dba})_{3}$ (22.9 mg, $25 \mu \mathrm{mol}, 10 \mathrm{~mol}-\%)$ and $\mathrm{AsPh}_{3}(18.7 \mathrm{mg}, 61 \mu \mathrm{mol}, 25 \mathrm{~mol}-\%)$. The mixture was stirred at $25^{\circ} \mathrm{C}$ for $24 \mathrm{~h}$. The mixture was diluted with aq. KF solution $(8 \mathrm{M}, 2.5 \mathrm{~mL})$ und it was sonicated in the ultra sound bath for $1 \mathrm{~min}$. The layers were separated and the organic layer was extracted with $\mathrm{Et}_{2} \mathrm{O}(3 \times 20 \mathrm{~mL})$. The combined organic layers were washed with brine $(10 \mathrm{~mL})$, tried over $\mathrm{Na}_{2} \mathrm{SO}_{4}$ and concentrated under reduced pressure. The residue was purified by flash chromatography $\left(c \mathrm{C}_{6} \mathrm{H}_{12}: \mathrm{AcOEt}=5: 1,2.0 \mathrm{~cm}, 20 \mathrm{~cm}, 10 \mathrm{~mL}\right)$. The polyen $(S, S, S)-33$ $(62.7 \mathrm{mg}, 51 \%, 87.7 \mu \mathrm{mol})$ was obtained as a pale brown oil. It represented a mixture of two rotamers in a keto and an enol form each [E-enol-(S,S,S)-33:Z-enol- $(S, S, S)-33: E$-keto- $(S, S, S)$ 33:Z-keto- $(S, S, S)-33=51: 2: 37: 9]$. This ratio was determined by integrating the 9-H signals [at $\delta=5.22$ (Z-keto), 5.37 (E-enol), 5.50 (E-keto) and 5.66 (Z-enol) ppm] and compared with the 3-OH signals [14.05 (E-enol) and 14.09 (Z-enol)].

The enantiomer $(R, R, R)$-33 was obtained analogously in $52 \%$ yield.

$\mathbf{R}_{\mathbf{f}}\left(c \mathrm{C}_{6} \mathrm{H}_{12}: \mathrm{AcOEt}=5: 1\right)=0.38$

E-enol-(S,S,S)-33:

${ }^{1} \mathrm{H}$ NMR $\left(500.10 \mathrm{MHz}, \mathrm{CDCl}_{3} / \mathrm{CHCl}_{3}\right.$, sample contained 1 weight- $\%$ of $c \mathrm{C}_{6} \mathrm{H}_{12}$ and 9 weight-\%

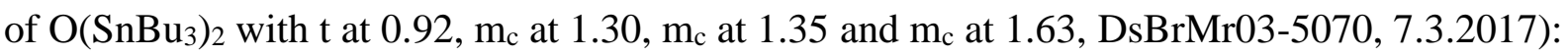
$\delta=0.17$ [s, 6H, Si- $\left.\left(\mathrm{CH}_{3}\right)_{2}\right], 0.81-0.86\left(\mathrm{~m}, 3 \mathrm{H}, 12-\mathrm{CH}_{3}\right), 0.84\left(\mathrm{t}, J_{14,13}=6.1 \mathrm{~Hz}, 3 \mathrm{H}, 14-\mathrm{H}_{3}\right)$, 0.95-0.99 (m, 3-H, 10- $\left.\mathrm{CH}_{3}\right), 0.96$ [s, 9H, Si-C( $\left.\left.\mathrm{CH}_{3}\right)_{3}\right], 1.06-1.17\left(\mathrm{~m}, 1 \mathrm{H}, 11-\mathrm{H}^{\mathrm{A}}\right), 1.06-1.17$ (m, $\left.1 \mathrm{H}, 13-\mathrm{H}^{\mathrm{A}}\right), 1.19-1.40(\mathrm{~m}, 1 \mathrm{H}, 12-\mathrm{H}), 1.19-1.40\left(\mathrm{~m}, 1 \mathrm{H}, 13-\mathrm{H}^{\mathrm{B}}\right), 1.19-1.40\left(\mathrm{~m}, 1 \mathrm{H}, 11-\mathrm{H}^{\mathrm{B}}\right)$, $1.81\left(\mathrm{~s}, 3 \mathrm{H}, 8-\mathrm{CH}_{3}\right), 2.62\left(\mathrm{~m}_{\mathrm{c}}, 1 \mathrm{H}, 10-\mathrm{H}\right), \mathrm{AB}$ signal $\left(\delta_{\mathrm{A}}=3.12, \delta_{\mathrm{B}}=3.29, J_{\mathrm{AB}}=14.1 \mathrm{~Hz}\right.$ in

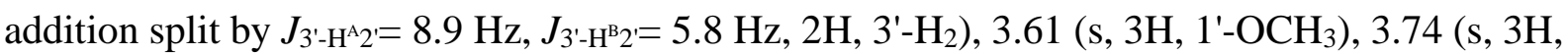

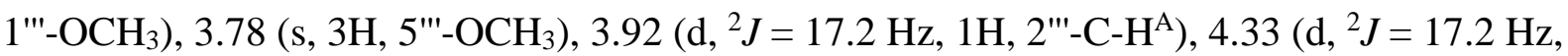
1H, 2'"-C-H $\left.{ }^{\mathrm{B}}\right), 4.44$ (m, 1H, 2'-H), 5.12 (s, 1H, 2-H), 5.37 (d, $\left.J_{9,10}=9.9 \mathrm{~Hz}, 1 \mathrm{H}, 9-\mathrm{H}\right), 5.81$ (d, $\left.J_{4,5}=15.0 \mathrm{~Hz}, 1 \mathrm{H}, 4-\mathrm{H}\right), 6.16\left(\mathrm{dd}, J_{6,5}=11.1 \mathrm{~Hz}, J_{6,7}=15.2 \mathrm{~Hz}, 1 \mathrm{H}, 6-\mathrm{H}\right), 6.34-6.41(\mathrm{~m}, 2 \mathrm{H}$, 4"'-H, 6"'-H), 6.46 (d, J7,6 = 15.2 Hz, 1H, 7-H), 6.70-6.74 (m, 2H, 2"-H, 6"-H), 6.92-7.03 (m, 3H, 3"-H, 5"-H, 3"'-H), 7.09 (dd, J5,6 = $\left.11.1 \mathrm{~Hz}, J_{5,4}=15.2 \mathrm{~Hz}, 1 \mathrm{H}, 5-\mathrm{H}\right), 14.05$ (s, 1H, 3-OH).

${ }^{13} \mathrm{C}$ NMR $\left(125.75 \mathrm{MHz}, \mathrm{CDCl}_{3} / \mathrm{CHCl}_{3}\right.$, sample contained $c \mathrm{C}_{6} \mathrm{H}_{12}$ and $\mathrm{O}\left(\mathrm{SnBu}_{3}\right)_{2}$ at 13.6, 17.6, 26.9 and 27.9, DsBrMz03-5071, 7.3.2017): $\delta=-4.4\left[\mathrm{Si}-\left(\mathrm{CH}_{3}\right)_{2}\right], 11.4(\mathrm{C}-14), 12.5\left(8-\mathrm{CH}_{3}\right)$, $18.3\left[\mathrm{Si}-\mathrm{C}\left(\mathrm{CH}_{3}\right)_{3}\right], 19.2\left(12-\mathrm{CH}_{3}\right), 21.5\left(10-\mathrm{CH}_{3}\right), 25.8$ [Si-C $\left.\left(\mathrm{CH}_{3}\right)_{3}\right], 30.2(\mathrm{C}-13), 30.7(\mathrm{C}-10)$, 32.4 (C-12), 34.8 (C-3'), 44.8 (C-11), 46.8 (2'"-C), 52.2 (1'- $\left.\mathrm{OCH}_{3}\right), 55.2\left(1 " '-\mathrm{OCH}_{3}\right), 55.4$ (5'"- 
OCH 3 ), 60.7 (C-2'), 90.0 (C-2), 98.3 (C-4"'), 103.8 (C-6"'), 116.8 (C-2'"), 120.2 (C-2", C-6"), 124.9 (C-6), 125.3 (C-4), 129.3 (C-3"'), 130.3 (C-3", C-5"), 130.4 (C-4"), 132.4 (C-8), 136.9 (C-5), 143.3 (C-7), 143.7 (C-9), 154.4 (C-1"), 157.9 (C-1'"), 160.5 (C-5"'), 169.7 (C-3), 171.4 (C-1'), $172.9(\mathrm{C}-1)$.

edHSQC [,,short-range H,C-COSY“, $\left.500.10 \mathrm{MHz} / 125.75 \mathrm{MHz}, \mathrm{CDCl}_{3}\right] ; \delta\left({ }^{1} \mathrm{H}\right) \leftrightarrow \delta\left({ }^{13} \mathrm{C}\right)$ : $0.17\left[\mathrm{~s}, \mathrm{Si}-\left(\mathrm{CH}_{3}\right)_{2}\right] \leftrightarrow-4.4\left[\mathrm{Si}-\left(\mathrm{CH}_{3}\right)_{2}\right], 0.81-0.86\left(\mathrm{~m}, 12-\mathrm{CH}_{3}\right) \leftrightarrow 19.2\left(12-\mathrm{CH}_{3}\right), 0.84(\mathrm{t}, 14-$ $\left.\mathrm{H}_{3}\right) \leftrightarrow 11.4(\mathrm{C}-14), 0.95-0.99\left(\mathrm{~m}, 10-\mathrm{CH}_{3}\right) \leftrightarrow 21.5\left(10-\mathrm{CH}_{3}\right), 0.96\left[\mathrm{~s}, \mathrm{Si}-\mathrm{C}\left(\mathrm{CH}_{3}\right)_{3}\right] \leftrightarrow 25.8[\mathrm{Si}-$ $\left.\mathrm{C}\left(\mathrm{CH}_{3}\right)_{3}\right], 1.06-1.17\left(\mathrm{~m}, 11-\mathrm{H}^{\mathrm{A}}\right) \leftrightarrow 44.8(\mathrm{C}-11), 1.06-1.17\left(\mathrm{~m}, 13-\mathrm{H}^{\mathrm{A}}\right) \leftrightarrow 30.2$ (13-C), 1.19$1.40(\mathrm{~m}, 12-\mathrm{H}) \leftrightarrow 32.4(\mathrm{C}-12), 1.19-1.40\left(\mathrm{~m}, 13-\mathrm{H}^{\mathrm{B}}\right) \leftrightarrow 30.2(\mathrm{C}-13), 1.19-1.40\left(\mathrm{~m}, 11-\mathrm{H}^{\mathrm{B}}\right) \leftrightarrow$ $44.8(\mathrm{C}-11), 1.81\left(\mathrm{~s}, 8-\mathrm{CH}_{3}\right) \leftrightarrow 12.5\left(8-\mathrm{CH}_{3}\right), 2.62\left(\mathrm{~m}_{\mathrm{c}}, 10-\mathrm{H}\right) \leftrightarrow 30.7(\mathrm{C}-10)$, AB signal $\left(\delta_{\mathrm{A}}=3.12, \delta_{\mathrm{B}}=3.29,3^{\prime}-\mathrm{H}_{2}\right) \leftrightarrow 34.8(\mathrm{C}-3$ ' $), 3.58\left(\mathrm{~s}, 1^{\prime}-\mathrm{OCH}_{3}\right) \leftrightarrow 52.2\left(1^{\prime}-\mathrm{OCH}_{3}\right), 3.74\left(\mathrm{~s}, 1^{\prime \prime \prime}-\right.$ $\left.\mathrm{OCH}_{3}\right) \leftrightarrow 55.2\left(1 "\right.$ '- $\left.-\mathrm{OCH}_{3}\right), 3.78\left(\mathrm{~s}, 5^{\prime \prime \prime}-\mathrm{OCH}_{3}\right) \leftrightarrow 55.4\left(5^{\prime \prime}-\mathrm{OCH}_{3}\right), 3.92\left(\mathrm{~d}, 2\right.$ '"-C-H $\left.{ }^{\mathrm{A}}\right) \leftrightarrow 46.8$ (2"'-C), 4.33 (d, 2"'-C-H $) \leftrightarrow 46.8$ (2'"-C), 4.44 (m, 2'-H) 60.7 (C-2'), 5.12 (s, 2-H) 90.0 (C-2), $5.37(\mathrm{~d}, 9-\mathrm{H}) \leftrightarrow 143.7(\mathrm{C}-9), 5.81(\mathrm{~d}, 4-\mathrm{H}) \leftrightarrow 125.3(\mathrm{C}-4), 6.16(\mathrm{dd}, 6-\mathrm{H}) \leftrightarrow 124.9(\mathrm{C}-$

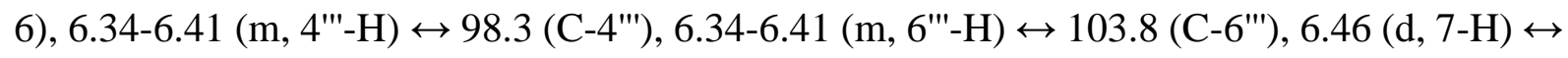
143.3 (C-7), 6.70-6.74 (m, 2"-H, 6"-H) ↔ 120.2 (C-2", C-6"), 6.92-7.03 (m, 3"-H, 5"-H) ↔ 130.3 (C-3", C-5"), 6.92-7.03 (m, 3"'-H) ↔ 129.3 (C-3"'), 7.09 (dd, 5-H) ↔ 136.9 (C-5).

\section{E-keto- $(S, S, S)-33$ :}

${ }^{1} \mathbf{H}$ NMR $\left(500.10 \mathrm{MHz}, \mathrm{CDCl}_{3} / \mathrm{CHCl}_{3}\right.$, sample contained 1 weight- $\%$ of $c \mathrm{C}_{6} \mathrm{H}_{12}$ and 9 weight-\%

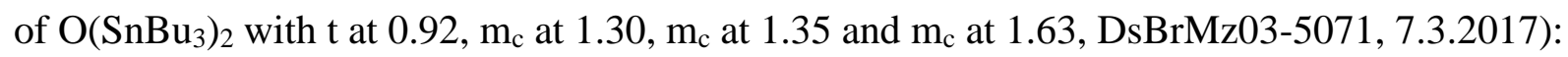
$\delta=0.17$ [s, 6H, Si- $\left.\left(\mathrm{CH}_{3}\right)_{2}\right], 0.81-0.86\left(\mathrm{~m}, 3 \mathrm{H}, 12-\mathrm{CH}_{3}\right), 0.84\left(\mathrm{t}, J_{14,13}=6.1 \mathrm{~Hz}, 3 \mathrm{H}, 14-\mathrm{H}_{3}\right)$, 0.95-0.99 (m, 3H, 10- $\left.\mathrm{CH}_{3}\right), 0.98$ [s, 9H, Si-C $\left.\left(\mathrm{CH}_{3}\right)_{3}\right], 1.06-1.17\left(\mathrm{~m}, 1 \mathrm{H}, 11-\mathrm{H}^{\mathrm{A}}\right), 1.06-1.17(\mathrm{~m}$, $\left.1 \mathrm{H}, 13-\mathrm{H}^{\mathrm{A}}\right), 1.19-1.40(\mathrm{~m}, 1 \mathrm{H}, 12-\mathrm{H}), 1.19-1.40\left(\mathrm{~m}, 1 \mathrm{H}, 13-\mathrm{H}^{\mathrm{B}}\right), 1.19-1.40\left(\mathrm{~m}, 1 \mathrm{H}, 11-\mathrm{H}^{\mathrm{B}}\right)$, $1.77\left(\mathrm{~s}, 3 \mathrm{H}, 8-\mathrm{CH}_{3}\right), 2.62\left(\mathrm{~m}_{\mathrm{c}}, 1 \mathrm{H}, 10-\mathrm{H}\right), \mathrm{AB}$ signal $\left(\delta_{\mathrm{A}}=3.12, \delta_{\mathrm{B}}=3.29, J_{\mathrm{AB}}=14.1 \mathrm{~Hz}\right.$ in addition split by $\left.J_{3^{\prime}-\mathrm{H}^{\mathrm{A}} 2^{\prime}}=8.9 \mathrm{~Hz}, J_{3^{\prime}-\mathrm{H}^{\mathrm{A}} 2^{\prime}}=5.8 \mathrm{~Hz}, 2 \mathrm{H}, 3^{\prime}-\mathrm{H}_{2}\right), 3.58\left(\mathrm{~s}, 3 \mathrm{H}, 1^{\prime}-\mathrm{OCH}_{3}\right), 3.71(\mathrm{~d}$,

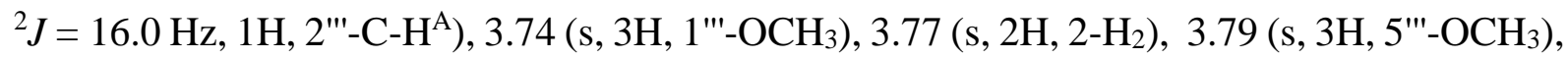
$4.03(\mathrm{~m}, 1 \mathrm{H}, 2 \mathrm{\prime}-\mathrm{H}), 4.36-4.40\left(\mathrm{~m}, 1 \mathrm{H}, 2^{2 '}-\mathrm{C}-\mathrm{H}^{\mathrm{B}}\right), 5.49$ (d, J9,10 = 9.7 Hz, 1H, 9-H), 6.27 (dd, $\left.J_{6,5}=11.0 \mathrm{~Hz}, J_{6,7}=15.0 \mathrm{~Hz}, 1 \mathrm{H}, 6-\mathrm{H}\right), 6.32\left(\mathrm{~d}, J_{4,5}=15.3 \mathrm{~Hz}, 1 \mathrm{H}, 4-\mathrm{H}\right), 6.34-6.41$ (m, 2H, 4'"H, 6"'-H), 6.66 (d, J7,6 = 15.2 Hz, 1H, 7-H), 6.70-6.74 (m, 2H, 2"-H, 6"-H), 6.92-7.03 (m, 3H, 3"-H, 5"-H, 3'"-H), 7.35 (dd, J5,6 $\left.=10.8 \mathrm{~Hz}, J_{5,4}=15.0 \mathrm{~Hz}, 1 \mathrm{H}, 5-\mathrm{H}\right)$.

${ }^{13} \mathrm{C} \mathrm{NMR}\left(125.75 \mathrm{MHz}, \mathrm{CDCl}_{3} / \mathrm{CHCl}_{3}\right.$, sample contained $c \mathrm{C}_{6} \mathrm{H}_{12}$ and $\mathrm{O}\left(\mathrm{SnBu}_{3}\right)_{2}$ at 13.6, 17.6, 26.9 and 27.9, DsBrMz03-5071, 7.3.2017): $\delta=-4.3$ [Si- $\left.\left(\mathrm{CH}_{3}\right)_{2}\right], 11.4(\mathrm{C}-14), 12.5\left(8-\mathrm{CH}_{3}\right)$, 
$18.3\left[\mathrm{Si}-\mathrm{C}\left(\mathrm{CH}_{3}\right)_{3}\right], 19.2\left(12-\mathrm{CH}_{3}\right), 21.3\left(10-\mathrm{CH}_{3}\right), 25.8$ [Si-C $\left.\left(\mathrm{CH}_{3}\right)_{3}\right], 30.2(\mathrm{C}-13), 30.7(\mathrm{C}-10)$, 32.4 (C-12), 34.4 (C-3'), 44.7 (C-11), 48.1 (C-2), $49.0\left(2\right.$ '"- $\left.\mathrm{CH}_{2}\right), 52.1\left(1 '-\mathrm{OCH}_{3}\right), 55.2$ (1'"$\left.\mathrm{OCH}_{3}\right), 55.4$ (5'"-OCH 3$), 61.0(\mathrm{C}-2 '), 98.6$ (C-6"'), 103.8 (C-4'"), 116.8 (C-2"'), 120.1 (C-2", C6"), 124.2 (C-6), 127.4 (C-4), 128.5 (C-7), 129.3 (C-3"'), 130.4 (C-4"), 130.7 (C-3", C-5"), 132.5 (C-8), 145.6 (C-5), 146.5 (C-9), 154.3 (C-1"), 158.9 (C-1"'), 161.0 (C-5"') 167.6 (C-1'), 170.9 (C-1), 193.4 (C-3).

edHSQC [,,short-range H,C-COSY“, $\left.500.10 \mathrm{MHz} / 125.75 \mathrm{MHz}, \mathrm{CDCl}_{3}\right] ; \delta\left({ }^{1} \mathrm{H}\right) \leftrightarrow \delta\left({ }^{13} \mathrm{C}\right)$ : $0.17\left[\mathrm{~s}, \mathrm{Si}-\left(\mathrm{CH}_{3}\right)_{2}\right] \leftrightarrow-4.3\left[\mathrm{Si}-\left(\mathrm{CH}_{3}\right)_{2}\right], 0.81-0.86\left(\mathrm{~m}, 12-\mathrm{CH}_{3}\right) \leftrightarrow 19.2\left(12-\mathrm{CH}_{3}\right), 0.84$ (t, $14-$ $\left.\mathrm{H}_{3}\right) \leftrightarrow 11.4(\mathrm{C}-14), 0.95-0.99\left(\mathrm{~m}, 10-\mathrm{CH}_{3}\right) \leftrightarrow 21.3\left(10-\mathrm{CH}_{3}\right), 0.96\left[\mathrm{~s}, \mathrm{Si}-\mathrm{C}\left(\mathrm{CH}_{3}\right)_{3}\right] \leftrightarrow 25.8[\mathrm{Si}-$ $\left.\mathrm{C}\left(\mathrm{CH}_{3}\right)_{3}\right], 1.06-1.17\left(\mathrm{~m}, 11-\mathrm{H}^{\mathrm{A}}\right) \leftrightarrow 44.7(\mathrm{C}-11), 1.06-1.17\left(\mathrm{~m}, 13-\mathrm{H}^{\mathrm{A}}\right) \leftrightarrow 30.2(\mathrm{C}-13), 1.19-$ $1.40(\mathrm{~m}, 12-\mathrm{H}) \leftrightarrow 32.4(\mathrm{C}-12), 1.19-1.40\left(\mathrm{~m}, 13-\mathrm{H}^{\mathrm{B}}\right) \leftrightarrow 30.2(\mathrm{C}-13), 1.19-1.40\left(\mathrm{~m}, 11-\mathrm{H}^{\mathrm{B}}\right) \leftrightarrow$ $44.7(\mathrm{C}-11), 1.77\left(\mathrm{~s}, 8-\mathrm{CH}_{3}\right) \leftrightarrow 12.5\left(8-\mathrm{CH}_{3}\right), 2.62\left(\mathrm{~m}_{\mathrm{c}}, 10-\mathrm{H}\right) \leftrightarrow 30.7$ (10-C), AB signal $\left(\delta_{\mathrm{A}}=3.12, \delta_{\mathrm{B}}=3.29,3^{\prime}-\mathrm{H}_{2}\right) \leftrightarrow 34.8(\mathrm{C}-3 '), 3.56\left(\mathrm{~s}, 1^{\prime}-\mathrm{OCH}_{3}\right) \leftrightarrow 52.1\left(1^{\prime}-\mathrm{OCH}_{3}\right), 3.71\left(\mathrm{~d}, 2^{\prime \prime}-\right.$

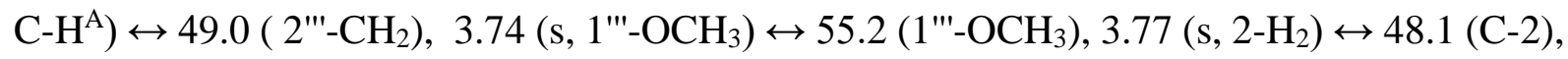
$3.79\left(\mathrm{~s}, 5^{\prime \prime}-\mathrm{OCH}_{3}\right) \leftrightarrow 55.4\left(5^{\prime \prime}-\mathrm{OCH}_{3}\right), 4.03\left(\mathrm{~m}, 2^{\prime}-\mathrm{H}\right), 61.0\left(\mathrm{C}-2^{\prime}\right), 4.36-4.40\left(\mathrm{~m}, 2^{2 '-}-\mathrm{C}-\mathrm{H}^{\mathrm{B}}\right) \leftrightarrow$ $49.0\left(2\right.$ '"- $\left.\mathrm{CH}_{2}\right), 5.49$ (d, J9,10 $\left.=9.7 \mathrm{~Hz}, 9-\mathrm{H}\right) \leftrightarrow 146.5(\mathrm{C}-9), 6.27$ (dd, 6-H) 124.2 (C-6), 6.32 $(\mathrm{d}, 4-\mathrm{H}) \leftrightarrow 127.4(\mathrm{C}-4), 6.34-6.41\left(\mathrm{~m}, 4{ }^{\prime \prime}-\mathrm{H}\right) \leftrightarrow 103.8$ (C-4'"), 6.34-6.41 (m, 6"'-H) 6"'), $6.66(\mathrm{~d}, 7-\mathrm{H}) \leftrightarrow 128.5$ (C-7), 6.70-6.74 (m, 2"-H, 6"-H) ↔ 120.4 (C-2", C-6"), 6.92-7.03 (m, 3"-H, 5"-H) ↔ 130.7 (C-3", C-5"), 6.92-7.03 (m, 3"'-H) ↔ 129.3 (C-3"'), 7.35 (dd, 5-H) $145.6(\mathrm{C}-5)$.

Specific rotation $(S, S, S)-33[a]_{D}^{20}=-204.7^{\circ}\left(\mathrm{c}=1.0\right.$ in $\left.\mathrm{CHCl}_{3}\right)$.

Specific rotation $(R, R, R)-33[a]_{D}^{20}=206.0^{\circ}\left(\mathrm{c}=1.0\right.$ in $\left.\mathrm{CHCl}_{3}\right)$.

HRMS (ESI) m/z: [M+H $]^{+}$Calcd for $\mathrm{C}_{42} \mathrm{H}_{62} \mathrm{O}_{7} \mathrm{NSi} 720.4290$; Found 720.4277

IR (KBr): $\tilde{v}=3025,2955,2930,2860,1745,1635,1610,1585,1575,1510,1465,1420,1390$, $1335,1290,1255,1210,1160,1120,1075,1035,990,915,840,805,780 \mathrm{~cm}^{-1}$. 


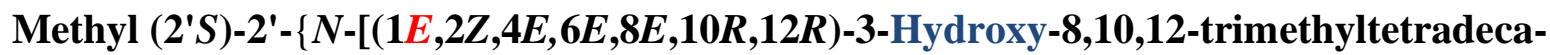

2,4,6,8-tetraenamido]- $N-(1 " ', 5$ '"-dimethoxybenzyl $)\}-3 '-\left\{1^{\prime \prime}-[(\right.$ tertbutyldimethylsilyl)oxy]phenyl $\}$ propanoate $[E-e n o l-(S, R, R)-33]$

in an equilibrium with

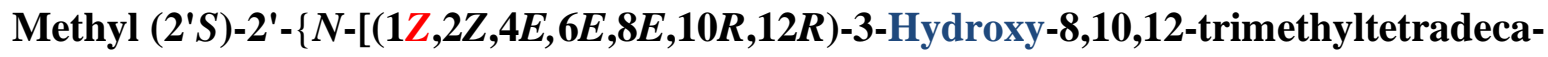
2,4,6,8-tetraenamido]-N-(1"',5'"-dimethoxybenzyl) $\}-3$ '- $\{1$ ''-[(tertbutyldimethylsilyl)oxy]phenyl $\}$ propanoate $[Z-e n o l-(S, R, R)-33]$ in an equilibrium with

Methyl (2'S)-2' - $\{N$-[(1E,4E,6E,8E,10R,12R)-3-Oxo-8,10,12-trimethyltetradeca-4,6,8trienamido]- $N-(1$ '",5"'-dimethoxybenzyl $)\}-3$ '-\{1"'-[(tertbutyldimethylsilyl)oxy]phenyl $\}$ propanoate $[$ E-keto-(S,R,R)-33] in an equilibrium with

Methyl (2'S)-2' - $\{N-[(1 Z, 4 E, 6 E, 8 E, 10 R, 12 R)-3-0 x 0-8,10,12$-trimethyltetradeca-4,6,8trienamido $]-N-\left(1{ }^{\prime \prime}, 5\right.$ '"'-dimethoxybenzyl $\left.)\right\}-3 '-\{1$ '-[(tertbutyldimethylsilyl)oxy]phenyl $\}$ propanoate $[Z-k e t o-(S, R, R)-33]$ and

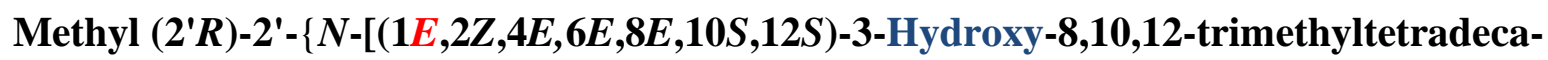
2,4,6,8-tetraenamido]-N-(1"',5'"-dimethoxybenzyl) $\}-3$ '- $\{1$ ''-[(tertbutyldimethylsilyl)oxy]phenyl $\}$ propanoate $[E-e n o l-(R, S, S)-33]$ in an equilibrium with

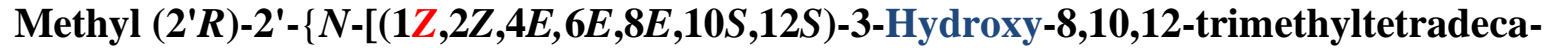
2,4,6,8-tetraenamido]- $N-\left(1^{\prime \prime}, 5\right.$ '"-dimethoxybenzyl) $\}-3$ '- $\left\{1^{\prime \prime}-[(\right.$ tertbutyldimethylsilyl)oxy]phenyl $\}$ propanoate $[Z$-enol-(R,S,S)-33] in an equilibrium with

Methyl (2'R)-2' - $\{N-[(1 E, 4 E, 6 E, 8 E, 10 S, 12 S)-3-0 x 0-8,10,12-t r i m e t h y l t e t r a d e c a-4,6,8-$ trienamido $]-N-(1 " ', 5$ "'-dimethoxybenzyl $)\}-3 '-\{1$ "'-[(tertbutyldimethylsilyl)oxy]phenyl $\}$ propanoate $[$ E-keto-(R,S,S)-33] 
Christian Drescher, Morris Keller, Olivier Potterat, Matthias Hamburger and Reinhard Brückner, Structure-Elucidating Total Synthesis of the (Polyenoyl)tetramic Acid Militarinone C

Methyl (2'R)-2' - $\{N-[(1 Z, 4 E, 6 E, 8 E, 10 S, 12 S)-3-0 \times 0-8,10,12-t r i m e t h y l t e t r a d e c a-4,6,8-$ trienamido]- $N-(1$ '",5'"'-dimethoxybenzyl $)\}-3$ '-\{1'-[(tertbutyldimethylsilyl)oxy]phenyl \} propanoate $[Z-k e t o-(R, S, S)-33]$ 

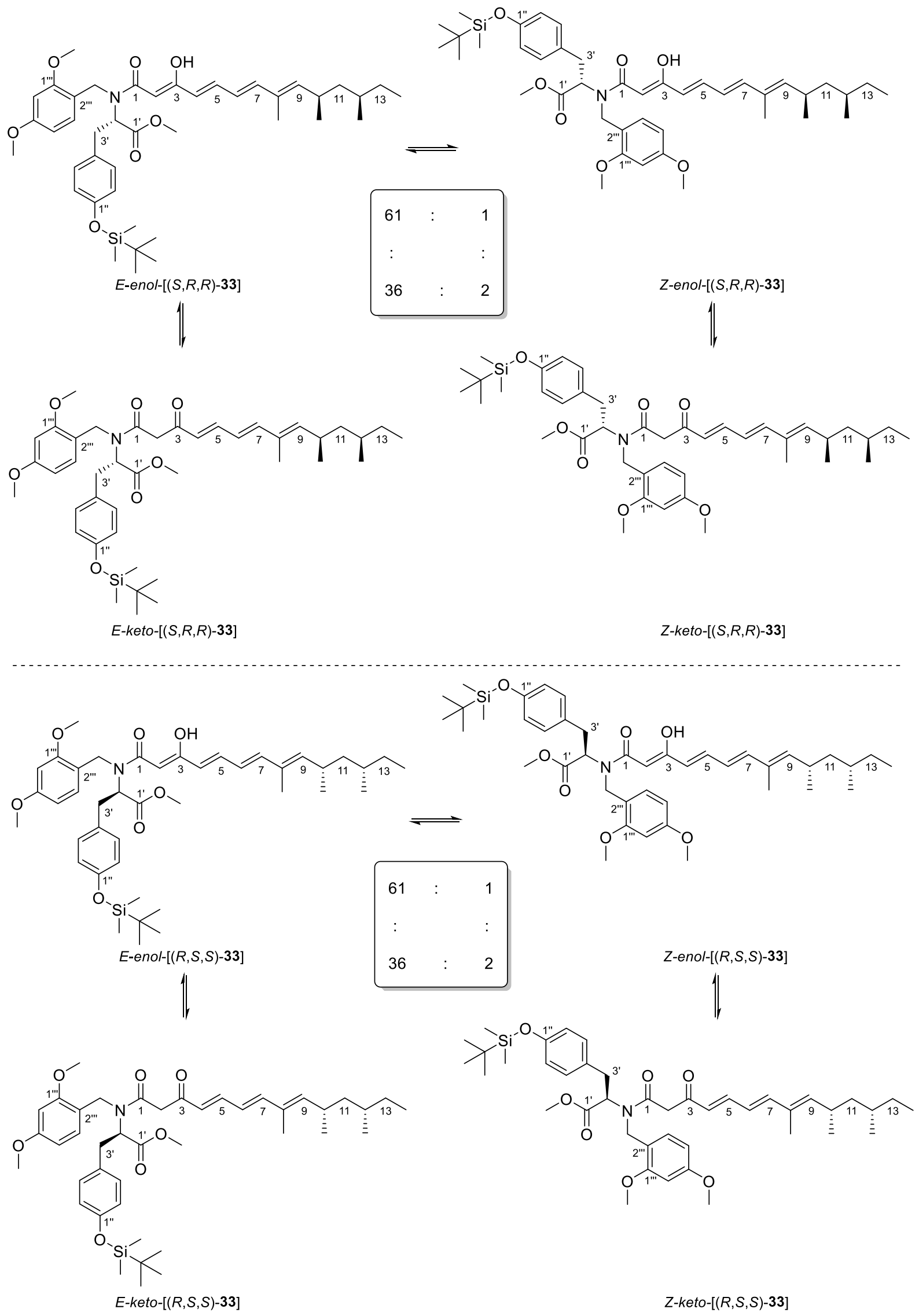

The alkyne $(R, R)-32(76.5 \mathrm{mg}, 418 \mu \mathrm{mol})$ was dissolved in THF $(2.6 \mathrm{~mL})$. It was added $\mathrm{Pd}\left(\mathrm{PPh}_{3}\right)_{2} \mathrm{Cl}_{2}(29.9 \mathrm{mg}, 43 \mu \mathrm{mol}, 10 \mathrm{~mol}-\%)$ and a solution of $\mathrm{Bu}_{3} \mathrm{SnH}(182 \mathrm{mg}, 624 \mu \mathrm{mol}$, 
1.5 eq. $)$ in THF $(1.7 \mathrm{~mL})$. The mixture was stirred at $25^{\circ} \mathrm{C}$ for $30 \mathrm{~min}$. Then a solution of $(S)-8$ (184 mg, $292 \mu \mathrm{mol}, 0.7$ eq.) in THF (2.6 mL) was added. Immediately it was added $\operatorname{Pd}_{2}(\mathrm{dba})_{3}$ (38.9 mg, $43 \mu \mathrm{mol}, 10 \mathrm{~mol}-\%)$ and $\mathrm{AsPh}_{3}(31.8 \mathrm{mg}, 104 \mu \mathrm{mol}, 25 \mathrm{~mol}-\%)$. The mixture was stirred at $25^{\circ} \mathrm{C}$ for $24 \mathrm{~h}$. The mixture was diluted with aq. $\mathrm{KF}$ solution $(8 \mathrm{M}, 4.5 \mathrm{~mL})$ and it was sonicated in the ultra sound bath for $1 \mathrm{~min}$. The layers were separated and the organic layer was extracted with $\mathrm{Et}_{2} \mathrm{O}(3 \times 20 \mathrm{~mL})$. The combined organic layers were washed with brine $(10 \mathrm{~mL})$, tried over $\mathrm{Na}_{2} \mathrm{SO}_{4}$ and concentrated under reduced pressure. The residue was purified by flash chromatography $\left(c \mathrm{C}_{6} \mathrm{H}_{12}: \mathrm{AcOEt}=5: 1,2.0 \mathrm{~cm}, 20 \mathrm{~cm}, 10 \mathrm{~mL}\right)$. The polyen $(S, S, S)-33$ (108.1 mg, 52\%, $150.2 \mu \mathrm{mol}$ ) was obtained as a pale brown oil. It represented a mixture of two rotamers in a keto and an enol form each $[$ E-enol- $(S, R, R)-33: Z$-enol- $(S, R, R)-33$ :E-keto- $(S, R, R)$ 33:Z-keto- $(S, R, R)-33=61: 1: 36: 2]$. This ratio was determined by integrating the $9-\mathrm{H}$ signals [at $\delta=5.23$ (Z-keto), 5.37 (E-enol), 5.50 (E-keto) and 5.55 (Z-enol) ppm] and compared with die 3-OH signals [14.06 (E-enol) and $14.10($ Z-enol) $]$.

The enantiomer $(R, S, S)$-33 was obtained analogously in $52 \%$ yield.

$\mathbf{R}_{\mathbf{f}}\left(c \mathrm{C}_{6} \mathrm{H}_{12}: \mathrm{AcOEt}=5: 1\right)=0.38$

E-enol-(S,R,R)-33:

${ }^{1} \mathbf{H}$ NMR $\left(500.10 \mathrm{MHz}, \mathrm{CDCl}_{3} / \mathrm{CHCl}_{3}\right.$, sample contained 4 weight- $\%$ of $\mathrm{O}\left(\mathrm{SnBu}_{3}\right)_{2}$ with $\mathrm{t}$ at $0.92, \mathrm{~m}_{\mathrm{c}}$ at $1.30, \mathrm{~m}_{\mathrm{c}}$ at 1.35 and $\mathrm{m}_{\mathrm{c}}$ at 1.63, DsBrNo29-500800, 29.11.2019): $\delta=0.18[\mathrm{~s}, 6 \mathrm{H}$, Si- $\left.\left(\mathrm{CH}_{3}\right)_{2}\right], 0.81-0.86\left(\mathrm{~m}, 3 \mathrm{H}, 12-\mathrm{CH}_{3}\right), 0.81-0.87$ (m, 3H, 14- $\left.\mathrm{H}_{3}\right), 0.95-0.99$ (m, 3H, 10- $\left.\mathrm{CH}_{3}\right)$, $0.98\left[\mathrm{~s}, 9 \mathrm{H}, \mathrm{Si}-\mathrm{C}\left(\mathrm{CH}_{3}\right)_{3}\right], 1.07-1.18\left(\mathrm{~m}, 1 \mathrm{H}, 11-\mathrm{H}^{\mathrm{A}}\right), 1.07-1.18\left(\mathrm{~m}, 1 \mathrm{H}, 13-\mathrm{H}^{\mathrm{A}}\right), 1.22-1.35(\mathrm{~m}$, $1 \mathrm{H}, 12-\mathrm{H}), 1.22-1.35\left(\mathrm{~m}, 1 \mathrm{H}, 13-\mathrm{H}^{\mathrm{B}}\right), 1.22-1.35\left(\mathrm{~m}, 1 \mathrm{H}, 11-\mathrm{H}^{\mathrm{B}}\right), 1.78\left(\mathrm{~d},{ }^{4} J_{8-\mathrm{CH}_{3}, 9}=1.0 \mathrm{~Hz}, 8-\right.$ $\left.\mathrm{CH}_{3}\right), 2.62\left(\mathrm{~m}_{\mathrm{c}}, 1 \mathrm{H}, 10-\mathrm{H}\right), \mathrm{AB}$ signal $\left(\delta_{\mathrm{A}}=3.12, \delta_{\mathrm{B}}=3.30, J_{\mathrm{AB}}=14.1 \mathrm{~Hz}\right.$ in addition split by $\left.J_{3^{\prime}-\mathrm{H}^{\mathrm{A}}, 2^{\prime}}=8.8 \mathrm{~Hz}, J_{3^{\prime}-\mathrm{H}^{\mathrm{B}}, 2^{\prime}}=6.4 \mathrm{~Hz}, 2 \mathrm{H}, 3^{\prime}-\mathrm{H}_{2}\right), 3.62\left(\mathrm{~s}, 3 \mathrm{H}, 1^{\prime}-\mathrm{OCH}_{3}\right), 3.76$ (s, 3H, 1''- $\left.-\mathrm{OCH}_{3}\right)$, 3.79 (s, 3H, 5"'-OCH $), 3.94\left(\mathrm{~d},{ }^{2} J=17.5 \mathrm{~Hz}, 1 \mathrm{H}, 2^{2}\right.$ '-C-H $\left.{ }^{\mathrm{A}}\right), 4.34$ (d, ${ }^{2} J=17.4 \mathrm{~Hz}, 1 \mathrm{H}, 2^{2 '}-\mathrm{C}-$

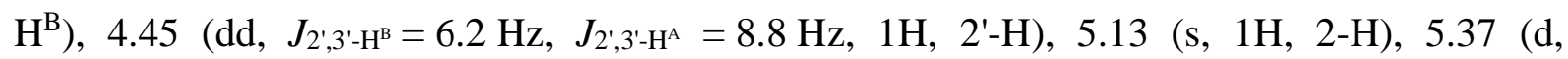
$\left.J_{9,10}=9.8 \mathrm{~Hz}, 1 \mathrm{H}, 9-\mathrm{H}\right), 5.83\left(\mathrm{~d}, J_{4,5}=15.1 \mathrm{~Hz}, 1 \mathrm{H}, 4-\mathrm{H}\right), 6.17\left(\mathrm{dd}, J_{6,5}=11.1 \mathrm{~Hz}\right.$, $\left.J_{6,7}=15.2 \mathrm{~Hz}, 1 \mathrm{H}, 6-\mathrm{H}\right), 6.34-6.41\left(\mathrm{~m}, 2 \mathrm{H}, 4 " '-\mathrm{H}, 6\right.$ '"-H), 6.46 (d, $\left.J_{7,6}=15.2 \mathrm{~Hz}, 1 \mathrm{H}, 7-\mathrm{H}\right), 6.71-$ 6.75 (m, 2H, 2"-H, 6"-H), 6.93-7.04 (m, 3H, 3"-H, 5"-H, 3"'-H), 7.10 (dd, J5,6=11.0 Hz, $\left.J_{5,4}=15.0 \mathrm{~Hz}, 1 \mathrm{H}, 5-\mathrm{H}\right), 14.06\left(\mathrm{~d},{ }^{4} J_{3-\mathrm{OH}, 2}=1.3 \mathrm{~Hz}, 1 \mathrm{H}, 3-\mathrm{OH}\right)$.

${ }^{13} \mathrm{C}$ NMR $\left(125.75 \mathrm{MHz}, \mathrm{CDCl}_{3} / \mathrm{CHCl}_{3}\right.$, sample contained $\mathrm{O}\left(\mathrm{SnBu}_{3}\right)_{2}$ at 13.6, 17.6, 26.9 and 27.9, DsBrNo29-500804, 29.11.2019): $\delta=-4.4$ [Si- $\left.\left(\mathrm{CH}_{3}\right)_{2}\right], 11.4(\mathrm{C}-14), 12.5\left(8-\mathrm{CH}_{3}\right), 18.3$ [Si- $\left.C\left(\mathrm{CH}_{3}\right)_{3}\right], 19.2\left(12-\mathrm{CH}_{3}\right), 21.5\left(10-\mathrm{CH}_{3}\right), 25.8\left[\mathrm{Si}-\mathrm{C}\left(\mathrm{CH}_{3}\right)_{3}\right], 30.2(\mathrm{C}-13), 30.7(\mathrm{C}-10), 32.4$ 
(C-12), 34.8 (C-3'), $44.8(\mathrm{C}-11), 46.8\left(2^{\prime \prime \prime}-\mathrm{CH}_{2}\right), 52.2\left(1^{\prime}-\mathrm{OCH}_{3}\right), 55.2\left(1{ }^{\prime \prime \prime}-\mathrm{OCH}_{3}\right), 55.4$ (5'"$\mathrm{OCH}_{3}$ ), 60.7 (C-2'), 89.9 (C-2), 98.3 (C-4"'), 103.8 (C-6"'), 116.8 (C-2'"), 120.2 (C-2", C-6"), 124.9 (C-6), 125.3 (C-4), 129.3 (C-3"'), 130.3 (C-3", C-5"), 130.4 (C-4"), 132.4 (C-8), 136.9 (C-5), 143.3 (C-7), 143.7 (C-9), 154.3 (C-1"), 157.9 (C-1'"), 160.5 (C-5"'), 169.7 (C-3), 171.7 $(\mathrm{C}-1$ '), $172.9(\mathrm{C}-1)$.

edHSQC [,,short-range H,C-COSY“, 500.10 MHz/125.75 MHz, $\left.\mathrm{CDCl}_{3}\right] ; \delta\left({ }^{1} \mathrm{H}\right) \leftrightarrow \delta\left({ }^{13} \mathrm{C}\right)$ : $0.18\left[\mathrm{~s}, \mathrm{Si}-\left(\mathrm{CH}_{3}\right)_{2}\right] \leftrightarrow-4.4\left[\mathrm{Si}-\left(\mathrm{CH}_{3}\right)_{2}\right], 0.81-0.86\left(\mathrm{~m}, 12-\mathrm{CH}_{3}\right) \leftrightarrow 19.2\left(12-\mathrm{CH}_{3}\right), 0.81-0.87(\mathrm{~m}$, $\left.14-\mathrm{H}_{3}\right) \leftrightarrow 11.4(\mathrm{C}-14), 0.95-0.99\left(\mathrm{~m}, 10-\mathrm{CH}_{3}\right) \leftrightarrow 21.5\left(10-\mathrm{CH}_{3}\right), 0.98\left[\mathrm{~s}, \mathrm{Si}-\mathrm{C}\left(\mathrm{CH}_{3}\right)_{3}\right] \leftrightarrow 25.8$ $\left[\mathrm{Si}-\mathrm{C}\left(\mathrm{CH}_{3}\right)_{3}\right], 1.07-1.18\left(\mathrm{~m}, 11-\mathrm{H}^{\mathrm{A}}\right) \leftrightarrow 44.8(\mathrm{C}-11), 1.07-1.18\left(\mathrm{~m}, 13-\mathrm{H}^{\mathrm{A}}\right) \leftrightarrow 30.2(\mathrm{C}-13), 1.22-$ $1.35(\mathrm{~m}, 12-\mathrm{H}) \leftrightarrow 32.4(\mathrm{C}-12), 1.22-1.35\left(\mathrm{~m}, 13-\mathrm{H}^{\mathrm{B}}\right) \leftrightarrow 30.2(\mathrm{C}-13), 1.22-1.35\left(\mathrm{~m}, 11-\mathrm{H}^{\mathrm{B}}\right) \leftrightarrow$ $44.8(\mathrm{C}-11), 1.78\left(\mathrm{~d}, 8-\mathrm{CH}_{3}\right) \leftrightarrow 12.5\left(8-\mathrm{CH}_{3}\right), \mathrm{AB}$ signal $\left(\delta_{\mathrm{A}}=3.12, \delta_{\mathrm{B}}=3.30,3^{\prime}-\mathrm{H}_{2}\right) \leftrightarrow 34.8$ $\left(\mathrm{C}-3\right.$ '), $3.62\left(\mathrm{~s}, 1^{\prime}-\mathrm{OCH}_{3}\right) \leftrightarrow 52.2\left(1^{\prime}-\mathrm{OCH}_{3}\right), 3.76\left(\mathrm{~s}, 11^{\prime \prime}-\mathrm{OCH}_{3}\right) \leftrightarrow 55.2(1 '$ '"-OCH $), 3.79$ (s,

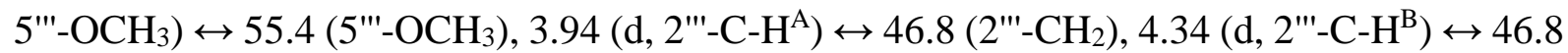
$\left(2^{\prime \prime}-\mathrm{CH}_{2}\right), 4.45\left(\mathrm{dd}, 2^{\prime}-\mathrm{H}\right) \leftrightarrow 60.7\left(\mathrm{C}-2^{\prime}\right), 5.13(\mathrm{~s}, 2-\mathrm{H}) \leftrightarrow 89.9(\mathrm{C}-2), 5.37(\mathrm{~d}, 9-\mathrm{H}) \leftrightarrow 143.7(\mathrm{C}-$ 9), $5.83(\mathrm{~d}, 4-\mathrm{H}) \leftrightarrow 125.3(\mathrm{C}-4), 6.17(\mathrm{dd}, 6-\mathrm{H}) \leftrightarrow 124.9$ (C-6), 6.34-6.41 (m, 4'"-H) 98.3 (C-4"'), 6.34-6.41 (m, 6"'-H) 103.8 (C-6'"), 6.46 (d, 7-H) ↔ 143.3 (C-7), 6.71-6.75 (m, 2"H, 6"-H) 120.2 (C-2", C-6"), 6.93-7.04 (m, 3"-H, 5"-H) ↔130.3 (C-3", C-5"), 6.93-7.04 (m, 3'"-H) $129.3\left(\mathrm{C}-3{ }^{\prime \prime}\right), 7.10(\mathrm{dd}, 5-\mathrm{H}) \leftrightarrow 136.9(\mathrm{C}-5)$,

\section{E-keto- $(S, R, R)-33:$}

${ }^{1} \mathbf{H}$ NMR $\left(500.10 \mathrm{MHz}, \mathrm{CDCl}_{3} / \mathrm{CHCl}_{3}\right.$, sample contained 4 weight- $\%$ of $\mathrm{O}\left(\mathrm{SnBu}_{3}\right)_{2}$ with $\mathrm{t}$ at 0.92, $\mathrm{m}_{\mathrm{c}}$ at 1.30, $\mathrm{m}_{\mathrm{c}}$ at 1.35 and $\mathrm{m}_{\mathrm{c}}$ at 1.63, DsBrNo29-500800, 29.11.2019): $\delta=0.18[\mathrm{~s}, 6 \mathrm{H}$, $\left.\mathrm{Si}-\left(\mathrm{CH}_{3}\right)_{2}\right], 0.81-0.86\left(\mathrm{~m}, 3 \mathrm{H}, 12-\mathrm{CH}_{3}\right), 0.81-0.87$ (m, 3H, 14- $\left.\mathrm{H}_{3}\right), 0.95-0.99\left(\mathrm{~m}, 3 \mathrm{H}, 10-\mathrm{CH}_{3}\right)$, 0.99 [s, 9H, Si-C $\left.\left(\mathrm{CH}_{3}\right)_{3}\right], 1.07-1.18\left(\mathrm{~m}, 1 \mathrm{H}, 11-\mathrm{H}^{\mathrm{A}}\right), 1.07-1.18\left(\mathrm{~m}, 1 \mathrm{H}, 13-\mathrm{H}^{\mathrm{A}}\right), 1.22-1.35(\mathrm{~m}$, $1 \mathrm{H}, 12-\mathrm{H}), 1.22-1.35\left(\mathrm{~m}, 1 \mathrm{H}, 13-\mathrm{H}^{\mathrm{B}}\right), 1.22-1.35\left(\mathrm{~m}, 1 \mathrm{H}, 11-\mathrm{H}^{\mathrm{B}}\right), 1.82\left(\mathrm{~d},{ }^{4} \mathrm{~J}_{8-\mathrm{CH}_{3}, 9}=1.1 \mathrm{~Hz}, 8-\right.$ $\left.\mathrm{CH}_{3}\right), 2.62\left(\mathrm{~m}_{\mathrm{c}}, 1 \mathrm{H}, 10-\mathrm{H}\right), \mathrm{AB}$ signal $\left(\delta_{\mathrm{A}}=3.09, \delta_{\mathrm{B}}=3.30, J_{\mathrm{AB}}=14.1 \mathrm{~Hz}\right.$ in addition split by $\left.J_{3^{\prime}-\mathrm{H}^{\mathrm{A}}, 2^{\prime}}=9.0 \mathrm{~Hz}, J_{3^{\prime}-\mathrm{H}^{\mathrm{B}}, 2^{\prime}}=6.4 \mathrm{~Hz}, 2 \mathrm{H}, 3^{\prime}-\mathrm{H}_{2}\right), 3.59\left(\mathrm{~s}, 3 \mathrm{H}, 1^{\prime}-\mathrm{OCH}_{3}\right), 3.71\left(\mathrm{~d},{ }^{2} J=16.0 \mathrm{~Hz}, 1 \mathrm{H}\right.$,

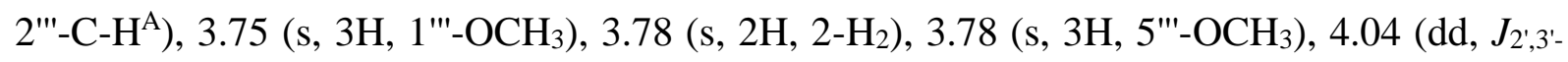
$\left.\mathrm{H}^{\mathrm{B}}=5.6 \mathrm{~Hz}, J_{2^{\prime}, 3^{\prime}-\mathrm{H}^{\mathrm{A}}}=9.1 \mathrm{~Hz}, 1 \mathrm{H}, 2^{\prime}-\mathrm{H}\right), 4.39\left(\mathrm{~d},{ }^{2} J=16.0 \mathrm{~Hz}, 1 \mathrm{H}, 2^{\prime \prime}-\mathrm{C}-\mathrm{H}^{\mathrm{B}}\right), 5.49(\mathrm{~d}$, $\left.J_{9,10}=9.8 \mathrm{~Hz}, 1 \mathrm{H}, 9-\mathrm{H}\right), 6.27\left(\mathrm{dd}, J_{6,5}=11.0 \mathrm{~Hz}, J_{6,7}=15.2 \mathrm{~Hz}, 1 \mathrm{H}, 6-\mathrm{H}\right), 6.33(\mathrm{~d}$, $\left.J_{4,5}=15.2 \mathrm{~Hz}, 1 \mathrm{H}, 4-\mathrm{H}\right), 6.35-6.41$ (m, 2H, 4"'-H, 6"'-H), 6.67 (d, $\left.J_{7,6}=14.9 \mathrm{~Hz}, 1 \mathrm{H}, 7-\mathrm{H}\right), 6.71-$ 6.74 (m, 2H, 2"-H, 6"-H), 6.93-7.03 (m, 3H, 3"-H, 5"-H, 3"'-H), 7.36 (dd, J5,6 = $11.1 \mathrm{~Hz}$, $\left.J_{5,4}=15.3 \mathrm{~Hz}, 1 \mathrm{H}, 5-\mathrm{H}\right)$. 
${ }^{13} \mathrm{C}$ NMR $\left(125.75 \mathrm{MHz}, \mathrm{CDCl}_{3} / \mathrm{CHCl}_{3}\right.$, sample contained $\mathrm{O}\left(\mathrm{SnBu}_{3}\right)_{2}$ at 13.6, 17.6, 26.9 and 27.9, DsBrNo29-500804, 29.11.2019): $\delta=-4.4\left[\mathrm{Si}-\left(\mathrm{CH}_{3}\right)_{2}\right], 11.4(\mathrm{C}-14), 12.54\left(8-\mathrm{CH}_{3}\right), 18.3$ [Si-C(CH$\left.)_{3}\right], 19.2\left(12-\mathrm{CH}_{3}\right), 21.3\left(10-\mathrm{CH}_{3}\right), 25.8$ [Si-C $\left.\left(\mathrm{CH}_{3}\right)_{3}\right], 30.1(\mathrm{C}-13), 30.9(\mathrm{C}-10), 32.4$

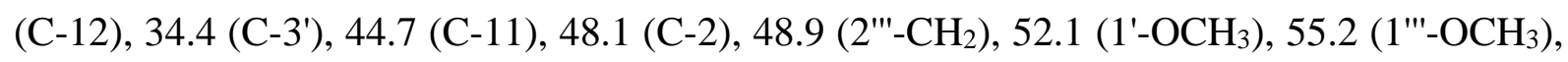
55.4 (5"'--OCH 3 ), 61.0 (C-2'), 98.6 (C-4"'), 103.8 (C-6"'), 116.2 (C-2"'), 120.1 (C-2", C-6"), 124.2 (C-6), 127.4 (C-4), 129.3 (C-3"'), 130.5 (C-4"), 130.7 (C-3", C-5"), 132.5 (C-8), 145.5 (C-5), 146.4 (C-9), 148.3 (C-7), 154.3 (C-1"), 158.8 (C-1"'), 161.0 (C-5"'), 167.6 (C-1'), 170.9 (C-1), $193.3(\mathrm{C}-3)$.

edHSQC [,,short-range H,C-COSY“, 500.10 MHz/125.75 MHz, $\left.\mathrm{CDCl}_{3}\right] ; \delta\left({ }^{1} \mathrm{H}\right) \leftrightarrow \delta\left({ }^{13} \mathrm{C}\right)$ : $0.18\left[\mathrm{~s}, \mathrm{Si}-\left(\mathrm{CH}_{3}\right)_{2}\right] \leftrightarrow-4.4\left[\mathrm{Si}-\left(\mathrm{CH}_{3}\right)_{2}\right], 0.81-0.86\left(\mathrm{~m}, 12-\mathrm{CH}_{3}\right) \leftrightarrow 19.2\left(12-\mathrm{CH}_{3}\right), 0.81-0.87(\mathrm{~m}$, $\left.14-\mathrm{H}_{3}\right) \leftrightarrow 11.4(\mathrm{C}-14), 0.95-0.99\left(\mathrm{~m}, 10-\mathrm{CH}_{3}\right) \leftrightarrow 21.3\left(10-\mathrm{CH}_{3}\right), 0.98\left[\mathrm{~s}, \mathrm{Si}-\mathrm{C}\left(\mathrm{CH}_{3}\right)_{3}\right] \leftrightarrow 25.8$ [Si-C $\left.\left(\mathrm{CH}_{3}\right)_{3}\right], 1.07-1.18\left(\mathrm{~m}, 11-\mathrm{H}^{\mathrm{A}}\right) \leftrightarrow 44.7(\mathrm{C}-11), 1.07-1.18\left(\mathrm{~m}, 13-\mathrm{H}^{\mathrm{A}}\right) \leftrightarrow 30.1(\mathrm{C}-13), 1.22-$ $1.35(\mathrm{~m}, 12-\mathrm{H}) \leftrightarrow 32.4(\mathrm{C}-12), 1.22-1.35\left(\mathrm{~m}, 13-\mathrm{H}^{\mathrm{B}}\right) \leftrightarrow 30.1(\mathrm{C}-13), 1.22-1.35\left(\mathrm{~m}, 11-\mathrm{H}^{\mathrm{B}}\right) \leftrightarrow$ $44.7(\mathrm{C}-11), 1.82\left(\mathrm{~d}, 8-\mathrm{CH}_{3}\right) \leftrightarrow 12.5\left(8-\mathrm{CH}_{3}\right), \mathrm{AB}$ signal $\left(\delta_{\mathrm{A}}=3.09, \delta_{\mathrm{B}}=3.30,3^{\prime}-\mathrm{H}_{2}\right) \leftrightarrow 34.4$ $\left(\mathrm{C}-3\right.$ '), $3.59\left(\mathrm{~s}, 1^{\prime}-\mathrm{OCH}_{3}\right) \leftrightarrow 52.1\left(1^{\prime}-\mathrm{OCH}_{3}\right), 3.71\left(\mathrm{~d}, 2\right.$ ''-C-H $\left.{ }^{\mathrm{A}}\right) \leftrightarrow 48.9\left(2^{\prime \prime}-\mathrm{CH}_{2}\right), 3.75$ (s, 1'"$\left.\mathrm{OCH}_{3}\right) \leftrightarrow 55.2\left(1\right.$ "'- $\left.-\mathrm{OCH}_{3}\right), 3.78\left(\mathrm{~s}, 2-\mathrm{H}_{2}\right) \leftrightarrow 48.1(\mathrm{C}-2), 3.78\left(\mathrm{~s}, 5^{\prime \prime}-\mathrm{OCH}_{3}\right) \leftrightarrow 55.4\left(5^{\prime \prime}-\mathrm{OCH}_{3}\right)$, $4.04\left(\mathrm{dd}, 2^{\prime}-\mathrm{H}\right) \leftrightarrow 61.0\left(\mathrm{C}-2^{\prime}\right), 4.39\left(\mathrm{~d}, 2^{\prime \prime}-\mathrm{C}-\mathrm{H}^{\mathrm{B}}\right) \leftrightarrow 48.9\left(2^{\prime \prime}-\mathrm{CH}_{2}\right), 5.49(\mathrm{~d}, 9-\mathrm{H}) \leftrightarrow 146.4(\mathrm{C}-$ 9), 6.27 (dd, 6-H) 124.2 (C-6), $6.33(\mathrm{~d}, 4-\mathrm{H}) \leftrightarrow 127.4$ (C-4), 6.35-6.41 (m, 4'"-H) 98.6 (C-4"'), 6.35-6.41 (m, 6"'-H) 103.8 (C-6"'), 6.67 (d, 7-H) ↔ 148.3 (C-7), 6.71-6.74 (m, 2"H, 6"-H) 120.1 (C-2", C-6"), 6.93-7.03 (m, 3"-H, 5"-H) ↔ 130.7 (C-3", C-5"), 6.93-7.03 (m, $3^{\prime \prime \prime-H)} 129.3\left(\mathrm{C}-3{ }^{\prime \prime \prime}\right), 7.36(\mathrm{dd}, 5-\mathrm{H}) \leftrightarrow 145.5(\mathrm{C}-5)$.

Specific rotation $(S, R, R)-33[a]_{D}^{20}=-220.0^{\circ}\left(\mathrm{c}=1.0\right.$ in $\left.\mathrm{CHCl}_{3}\right)$.

Specific rotation $(R, S, S)-33[a]_{D}^{20}=224.0^{\circ}\left(\mathrm{c}=1.0\right.$ in $\left.\mathrm{CHCl}_{3}\right)$.

HRMS (ESI) m/z: [M+H $]^{+}$Calcd for $\mathrm{C}_{42} \mathrm{H}_{62} \mathrm{O}_{7} \mathrm{NSi} 720.4290$; Found 720.4277

IR (KBr): $\tilde{v}=3030,2960,2930,2860,1745,1635,1610,1585,1575,1510,1465,1420,1390$, $1335,1290,1255,1160,1120,1075,1035,915,840,805,780 \mathrm{~cm}^{-1}$. 
Christian Drescher, Morris Keller, Olivier Potterat, Matthias Hamburger and Reinhard Brückner, Structure-Elucidating Total Synthesis of the (Polyenoyl)tetramic Acid Militarinone C

(5S)-5-(1'-Hydroxybenzyl)-1-(1'",5'"'-dimethoxybenzyl)-3-(1'Z,2' $\left.E, 4^{\prime} E, 6 ' E, 8^{\prime} S, 10 ' S\right)-1^{\prime}-$ hydroxy-6',8',10'-trimethyldodeca-2',4',6'-trien-1'-ylidene)pyrrolidine-2,4-dion [Z-enol$(S, S, S)-34]$

in an 66:34 mixture with

(5S)-5-(1"'-Hydroxybenzyl)-1-(1'",5'"'-dimethoxybenzyl)-3-(1'E,2'E,4' $E, 6$ ' $\left.E, 8^{\prime} S, 10 ' S\right)-1$ 'hydroxy-6',8',10'-trimethyldodeca-2',4',6' -trien-1'-ylidene)pyrrolidine-2,4-dion [E-enol$(S, S, S)-34]$

and

(5R)-5-(1"-Hydroxybenzyl)-1-(1'",5'"'-dimethoxybenzyl)-3-(1'Z,2' $\left.E, 4^{\prime} E, 6^{\prime} E, 8^{\prime} R, 10 ' R\right)-1^{\prime}-$ hydroxy-6',8',10'-trimethyldodeca-2',4',6'-trien-1' -ylidene)pyrrolidine-2,4-dion [Z-enol$(\boldsymbol{R}, \boldsymbol{R}, \boldsymbol{R})-34]$

in an 66:34 mixture with

(5R)-5-(1'-Hydroxybenzyl)-1-(1'"',5'"'-dimethoxybenzyl)-3-(1'E,2' $\left.E, 4^{\prime} E, 6^{\prime} E, 8^{\prime} R, 10 ' R\right)-1$ 'hydroxy-6',8',10'-trimethyldodeca-2',4',6' -trien-1'-ylidene)pyrrolidine-2,4-dion [E-enol$(\boldsymbol{R}, \boldsymbol{R}, \boldsymbol{R})-34]$
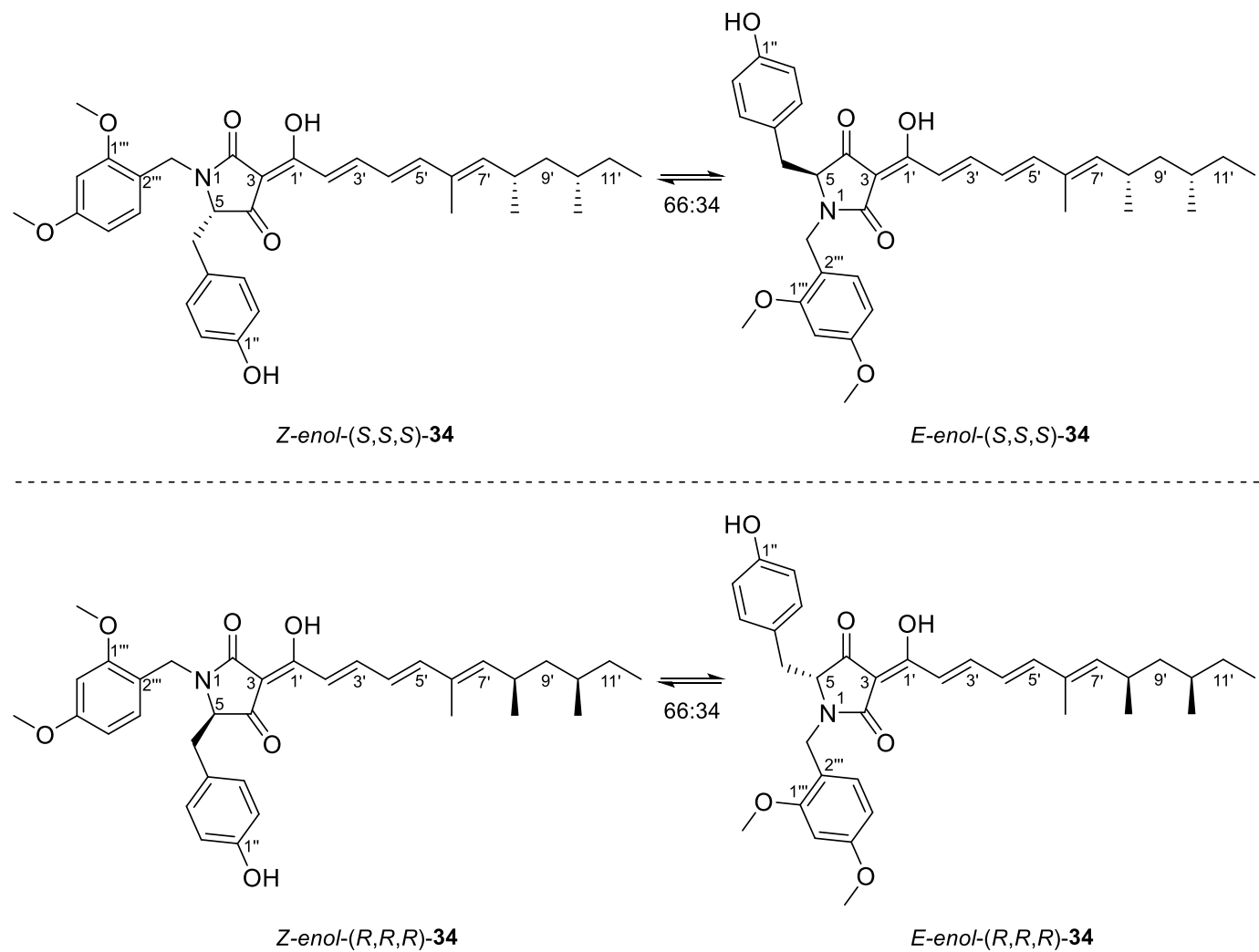
To a solution of the polyene $(S, S, S)-33(27.0 \mathrm{mg}, 38.0 \mu \mathrm{mol})$ in THF $(0.3 \mathrm{~mL})$ was added TBAF ( $1 \mathrm{M}$ in THF, $135 \mu \mathrm{L}, 133 \mu \mathrm{mol}, 3.5$ eq.) at $25^{\circ} \mathrm{C}$. At this temperature the mixture was stirred for $1 \mathrm{~h}$. The solvent was removed under reduced pressure and the residue was obtained with $\mathrm{H}_{2} \mathrm{O}(1.0 \mathrm{~mL})$, aq. $\mathrm{H}_{2} \mathrm{SO}_{4}(4 \mathrm{M}, 1.0 \mathrm{~mL})$ and $\mathrm{Et}_{2} \mathrm{O}(5 \mathrm{~mL})$. The layers were separated and the organic layer was extracted with $\mathrm{Et}_{2} \mathrm{O}(3 \times 10 \mathrm{~mL})$. The combined organic layers were tried over $\mathrm{Na}_{2} \mathrm{SO}_{4}$ and concentrated under reduced pressure. The residue was purified by reverse phase flash chromatography $\left(\mathrm{MeCN}: \mathrm{H}_{2} \mathrm{O}: \mathrm{TFA}=85: 15: 0.1,1.5 \mathrm{~cm}, 10 \mathrm{~cm}, 2 \mathrm{~mL}\right)$. The tetramic acid $(S, S, S)-34(12.9 \mathrm{mg}, 60 \%, 23.1 \mu \mathrm{mol})$ was obtained as a yellow powder (mp.: $\left.78-80^{\circ} \mathrm{C}\right)$. It was assigned as a 66:34 mixture of the $Z$ - and the $E$-enol of the title compounds $(S, S, S)-34$. This ratio was determined by integrating dd's of the respective 3'-H [7.37 (E-enol) and 7.45 (Zenol)].

The enantiomer $(R, R, R)-34$ was obtained analogously in $60 \%$ yield.

$\mathbf{R}_{\mathbf{f}}\left(\mathrm{MeCN}: \mathrm{H}_{2} \mathrm{O}: \mathrm{TFA}=85: 15: 0.1\right)=0.18$

Z-enol-(S,S,S)-34

${ }^{1} \mathbf{H}$ NMR $\left(500.10 \mathrm{MHz}, \mathrm{CDCl}_{3} / \mathrm{CHCl}_{3}\right.$, sample contained unknown impurity s at 1.26 and br. s at 3.63, DsBrSe27-500400, 30.9.2019): $\delta=0.80-0.87$ (m, 3H, 10'- $\left.\mathrm{CH}_{3}\right), 0.80-0.87$ (m, 3H, 12'$\left.\mathrm{H}_{3}\right), 0.96\left(\mathrm{~d}, J_{8^{\prime}-\mathrm{CH}_{3}, 8^{\prime}}=6.6 \mathrm{~Hz}, 3 \mathrm{H}, 8^{\prime}-\mathrm{CH}_{3}\right), 1.08-1.16\left(\mathrm{~m}, 1 \mathrm{H}, 9^{\prime}-\mathrm{H}^{\mathrm{A}}\right), 1.08-1.16\left(\mathrm{~m}, 1 \mathrm{H}, 11^{\prime}-\right.$ $\left.\mathrm{H}^{\mathrm{A}}\right), 1.21-1.34\left(\mathrm{~m}, 1 \mathrm{H}, 10^{\prime}-\mathrm{H}\right), 1.21-1.34\left(\mathrm{~m}, 1 \mathrm{H}, 9^{\prime}-\mathrm{H}^{\mathrm{B}}\right), 1.19-1.40\left(\mathrm{~m}, 1 \mathrm{H}, 11^{\prime}-\mathrm{H}^{\mathrm{B}}\right), 1.80(\mathrm{~d}$, $\left.{ }^{4} J_{6}-\mathrm{CH}_{3}, 7^{\prime}=0.9 \mathrm{~Hz}, 3 \mathrm{H}, 66^{\prime}-\mathrm{CH}_{3}\right), 2.64\left(\mathrm{~m}_{\mathrm{c}}, 1 \mathrm{H}, 8^{\prime}-\mathrm{H}\right), 3.05-3.15\left(\mathrm{~m}, 2 \mathrm{H}, 5-\mathrm{CH}_{2}\right), 3.79$ (s, 3H, 1'"$\left.\mathrm{OCH}_{3}\right), 3.79$ (s, 3H, 5"'-OCH $), 3.83\left(\mathrm{~m}_{\mathrm{c}}, 1 \mathrm{H}, 5-\mathrm{H}\right), 4.21\left(\mathrm{~d},{ }^{2} J=14.7 \mathrm{~Hz}, 1 \mathrm{H}, 2^{2 "}-\mathrm{C}-\mathrm{H}^{\mathrm{A}}\right), 5.00$ $\left(\mathrm{d},{ }^{2} J=14.7 \mathrm{~Hz}, 1 \mathrm{H}, 2^{\prime \prime}-\mathrm{C}-\mathrm{H}^{\mathrm{B}}\right), 5.51\left(\mathrm{~d}, J_{7^{\prime}, 6^{\prime}}=9.7 \mathrm{~Hz}, 1 \mathrm{H}, 7^{\prime}-\mathrm{H}\right), 6.38\left(\mathrm{dd}, J_{4^{\prime}, 3^{\prime}}=11.1 \mathrm{~Hz}\right.$, $\left.J_{4^{\prime}, 5^{\prime}}=14.8 \mathrm{~Hz}, 1 \mathrm{H}, 4^{\prime}-\mathrm{H}\right), 6.42-6.45$ (m, 2H, 4'"-H, 6"'-H), 6.64 (d, $\left.J_{5^{\prime}, 4^{\prime}}=14.8 \mathrm{~Hz}, 1 \mathrm{H}, 5^{\prime}-\mathrm{H}\right)$, 6.64-6.68 (m, 2H, 2"-H, 6"-H), 6.92-6.97 (m, 2H, 3"-H, 5"-H), 7.08-7.14 (m, 1H, 3"'-H), 7.10 $\left(\mathrm{d}, J_{2^{\prime}, 3^{\prime}}=15.2 \mathrm{~Hz}, 1 \mathrm{H}, 2^{\prime}-\mathrm{H}\right), 7.45\left(\mathrm{dd}, J_{3^{\prime}, 4^{\prime}}=11.1 \mathrm{~Hz}, J_{3^{\prime}, 2^{\prime}}=15.2 \mathrm{~Hz}, 1 \mathrm{H}, 3^{\prime}-\mathrm{H}\right)$.

${ }^{13} \mathrm{C}$ NMR $\left(125.75 \mathrm{MHz}, \mathrm{CDCl}_{3} / \mathrm{CHCl}_{3}\right.$, sample contained unknown impurity at 14.2 and 22.8, DsBrSe27-500404, 30.9.2019): $\delta=11.4\left(\mathrm{C}-12^{\prime}\right), 12.5\left(6^{\prime}-\mathrm{CH}_{3}\right), 19.2\left(10^{\prime}-\mathrm{CH}_{3}\right), 21.3\left(8^{\prime}-\mathrm{CH}_{3}\right)$, 30.2 (C-11'), $31.0\left(\mathrm{C}-8\right.$ '), $32.4\left(\mathrm{C}-10\right.$ '), $34.1\left(5-\mathrm{CH}_{2}\right), 38.4\left(2^{\prime \prime}-\mathrm{CH}_{2}\right), 44.7(\mathrm{C}-9$ '), 55.5 (1'"$\left.\mathrm{OCH}_{3}\right), 55.5$ (5'"--OCH 3$), 65.1$ (C-5), 98.6 (C-6"'), 100.6 (C-3), 104.5 (C-4"'), 115.3 (C-2", C6"), 116.3 (C-2"'), 120.2 (C-2'), 125.2 (C-4'), 127.2 (C-4"), 130.9 (C-3", C-5"), 131.5 (C-3"'), 132.9 (C-6'), 145.8 (C-3'), 147.2 (C-7'), 148.7 (C-5'), 154.7 (C-1"), 158.7 (C-1'"), 160.9 (C-5'"), 173.2 (C-1'), 173.9 (C-2), 194.7 (C-4). 
edHSQC [,,short-range H,C-COSY“, 500.10 MHz/125.75 MHz, $\mathrm{CDCl}_{3} ; \delta\left({ }^{1} \mathrm{H}\right) \leftrightarrow \delta\left({ }^{13} \mathrm{C}\right)$ : 0.80-0.87 (m, 10'- $\left.\mathrm{CH}_{3}\right) \leftrightarrow 19.2\left(10^{\prime}-\mathrm{CH}_{3}\right), 0.80-0.87\left(\mathrm{~m}, 12^{\prime}-\mathrm{H}_{3}\right) \leftrightarrow 11.4\left(\mathrm{C}-12^{\prime}\right), 0.96\left(\mathrm{~d}, 8^{\prime}-\right.$ $\left.\mathrm{CH}_{3}\right) \leftrightarrow 21.3\left(8^{\prime}-\mathrm{CH}_{3}\right), 1.08-1.16\left(\mathrm{~m}, 9^{\prime}-\mathrm{H}^{\mathrm{A}}\right) \leftrightarrow 44.7\left(\mathrm{C}-9^{\prime}\right), 1.08-1.16\left(\mathrm{~m}, 11^{\prime}-\mathrm{H}^{\mathrm{A}}\right) \leftrightarrow 30.2(\mathrm{C}-$ $\left.11^{\prime}\right), 1.21-1.34\left(\mathrm{~m}, 10^{\prime}-\mathrm{H}\right) \leftrightarrow 32.4\left(\mathrm{C}-10^{\prime}\right), 1.21-1.34\left(\mathrm{~m}, 9^{\prime}-\mathrm{H}^{\mathrm{B}}\right) \leftrightarrow 44.7$ (C-9'), 1.19-1.40 (m, $\left.11^{\prime}-\mathrm{H}^{\mathrm{B}}\right) \leftrightarrow 30.2\left(\mathrm{C}-11^{\prime}\right), 1.80\left(\mathrm{~d}, 6^{\prime}-\mathrm{CH}_{3}\right) \leftrightarrow 12.5\left(6^{\prime}-\mathrm{CH}_{3}\right), 2.64\left(\mathrm{~m}_{\mathrm{c}}, 8^{\prime}-\mathrm{H}\right), \leftrightarrow 31.0\left(\mathrm{C}-8^{\prime}\right), 3.05-$ $3.15\left(\mathrm{~m}, 5-\mathrm{CH}_{2}\right) \leftrightarrow 34.1\left(5-\mathrm{CH}_{2}\right), 3.79\left(\mathrm{~s}, 1\right.$ '"-- $\left.\mathrm{OCH}_{3}\right) \leftrightarrow 55.5\left(1 "\right.$ '- $\left.-\mathrm{OCH}_{3}\right), 3.79(\mathrm{~s}, 5$ "'--OCH $)$ $\leftrightarrow 55.5\left(5 " '-\mathrm{OCH}_{3}\right), 3.83\left(\mathrm{~m}_{\mathrm{c}}, 5-\mathrm{H}\right) \leftrightarrow 65.1(\mathrm{C}-5), 4.21(\mathrm{~d}, 2$ "'-C-H $) \leftrightarrow 38.4\left(2^{\mathrm{A}}\right.$ '"-CH$), 5.00$ $\left(\mathrm{d}, 2^{\prime \prime}-\mathrm{C}-\mathrm{H}^{\mathrm{B}}\right) \leftrightarrow 38.4\left(2^{\prime \prime}-\mathrm{CH}_{2}\right), 5.51\left(\mathrm{~d}, 7^{\prime}-\mathrm{H}\right) \leftrightarrow 147.2\left(\mathrm{C}-7^{\prime}\right), 6.38\left(\mathrm{dd}, 4^{\prime}-\mathrm{H}\right) \leftrightarrow 125.2\left(\mathrm{C}-4^{\prime}\right)$, 6.42-6.45 (m, 4'"-H) ↔ 104.5 (C-4"'), 6.42-6.45 (m, 6"'-H) ↔ 98.6 (C-6"'), 6.64 (d, 5'-H) 130.8 (C-5'), 6.64-6.68 (m, 2H, 2"-H, 6"-H) ↔ 115.3 (C-2", C-6"), 6.92-6.97 (m, 2H, 3"-H, 5"H) 130.9 (C-3", C-5"), 7.08-7.14 (m, 3'"-H), ↔ 131.5 (C-3'"), 7.10 (d, 2'-H) 120.2 (C-2'), $7.45\left(\mathrm{dd}, 3^{\prime}-\mathrm{H}\right) \leftrightarrow 145.8\left(\mathrm{C}-3^{\prime}\right)$.

\section{E-enol-(S,S,S)-34}

${ }^{1} \mathbf{H}$ NMR $\left(500.10 \mathrm{MHz}, \mathrm{CDCl}_{3} / \mathrm{CHCl}_{3}\right.$, sample contained unknown impurity s at 1.26 and br. s at 3.63, DsBrSe27-500400, 30.9.2019): 0.80-0.87 (m, 3H, 10'- $\left.\mathrm{CH}_{3}\right), 0.80-0.87$ (m, 3H, 12'- $\left.\mathrm{H}_{3}\right)$, $0.97\left(\mathrm{~d}, J_{8^{\prime}-\mathrm{CH}_{3}, 8^{\prime}}=6.6 \mathrm{~Hz}, 3 \mathrm{H}, 8^{\prime}-\mathrm{CH}_{3}\right), 1.08-1.16\left(\mathrm{~m}, 1 \mathrm{H}, 9^{\prime}-\mathrm{H}^{\mathrm{A}}\right), 1.08-1.16\left(\mathrm{~m}, 1 \mathrm{H}, 11^{\prime}-\mathrm{H}^{\mathrm{A}}\right)$,

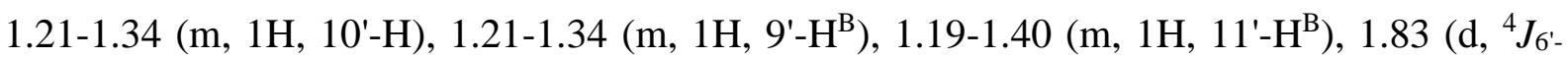
$\left.\mathrm{CH}_{3}, 7^{\prime}=1.0 \mathrm{~Hz}, 3 \mathrm{H}, 6 \mathrm{C}^{\prime}-\mathrm{CH}_{3}\right), 2.64\left(\mathrm{~m}_{\mathrm{c}}, 1 \mathrm{H}, 8^{\prime}-\mathrm{H}\right), 3.05-3.15\left(\mathrm{~m}, 2 \mathrm{H}, 5-\mathrm{CH}_{2}\right), 3.79$ (s, 3H, 1'"$\left.\mathrm{OCH}_{3}\right), 3.79$ (s, 3H, 5'"-OCH 3 ), $3.99\left(\mathrm{t}, J_{5,5-\mathrm{CH}_{2}}=4.3 \mathrm{~Hz}, 1 \mathrm{H}, 5-\mathrm{H}\right), 4.21\left(\mathrm{~d},{ }^{2} J=14.6 \mathrm{~Hz}, 1 \mathrm{H}\right.$, 2'"-C-H $\left.{ }^{\mathrm{A}}\right), 5.01\left(\mathrm{~d},{ }^{2} J=14.6 \mathrm{~Hz}, 1 \mathrm{H}, 2^{\prime \prime}-\mathrm{C}-\mathrm{H}^{\mathrm{B}}\right), 5.52\left(\mathrm{~d}, J_{7^{\prime}, 6^{\prime}}=9.9 \mathrm{~Hz}, 1 \mathrm{H}, 7^{\prime}-\mathrm{H}\right), 6.42-6.45$ (m, 2H, 4"'-H, 6'"-H), 6.56 (d, J', 3' = 14.8 Hz, 1H, 2'-H), 6.64-6.68 (m, 2H, 2"-H, 6"-H), 6.71 (dd, $\left.J_{4^{\prime}, 3^{\prime}}=J_{4^{\prime}, 5^{\prime}}=11.7 \mathrm{~Hz}, 1 \mathrm{H}, 4^{\prime}-\mathrm{H}\right), 6.82\left(\mathrm{~d}, J_{5^{\prime}, 4^{\prime}}=11.7 \mathrm{~Hz}, 1 \mathrm{H}, 5^{\prime}-\mathrm{H}\right), 6.92-6.97$ (m, 2H, 3"-H, 5"H), 7.08-7.14 (m, 1H, 3"'-H), 7.37 (dd, $\left.J_{3^{\prime}, 4^{\prime}}=11.8 \mathrm{~Hz}, J_{3^{\prime}, 2^{\prime}}=15.2 \mathrm{~Hz}, 1 \mathrm{H}, 3^{\prime}-\mathrm{H}\right)$.

${ }^{13} \mathrm{C} \mathrm{NMR}\left(125.75 \mathrm{MHz}, \mathrm{CDCl}_{3} / \mathrm{CHCl}_{3}\right.$, sample contained unknown impurity at 14.2 and 22.8, DsBrSe27-500404, 30.9.2019): $\delta=11.4\left(\mathrm{C}-12\right.$ '), 12.7 (6'- $\left.\mathrm{CH}_{3}\right), 19.2\left(10^{\prime}-\mathrm{CH}_{3}\right), 21.3\left(8^{\prime}-\mathrm{CH}_{3}\right)$, 30.2 (C-11'), $31.0\left(\mathrm{C}-8\right.$ '), 32.5 (C-10'), $34.0\left(5-\mathrm{CH}_{2}\right), 38.4\left(2^{\prime \prime \prime}-\mathrm{CH}_{2}\right), 44.7(\mathrm{C}-9$ '), 55.5 (1'"$\left.\mathrm{OCH}_{3}\right), 55.5$ (5'"-OCH 3 ), 65.0 (C-5), 98.6 (C-6"'), 100.6 (C-3), 104.5 (C-4"'), 115.3 (C-2", C6"), 115.9 (C-5'), 116.3 (C-2"'), 125.2 (C-3'), 127.2 (C-4"), 130.8 (C-3", C-5"), 131.5 (C-3"'), 133.2 (C-6'), 147.2 (C-4'), 147.3 (C-7'), 149.8 (C-2'), 154.7 (C-1"), 158.6 (C-1'"), 160.7 (C-5'"), 174.3 (C-1'), 175.2 (C-2), 194.2 (C-4).

edHSQC [,,short-range H,C-COSY“, 500.10 MHz/125.75 MHz, $\mathrm{CDCl}_{3} ; \delta\left({ }^{1} \mathrm{H}\right) \leftrightarrow \delta\left({ }^{13} \mathrm{C}\right)$ : 0.80-0.87 (m, 10'- $\left.\mathrm{CH}_{3}\right) \leftrightarrow 19.2\left(10^{\prime}-\mathrm{CH}_{3}\right), 0.80-0.87\left(\mathrm{~m}, 12^{\prime}-\mathrm{H}_{3}\right) \leftrightarrow 11.4\left(\mathrm{C}-12^{\prime}\right), 0.96\left(\mathrm{~d}, 8^{\prime}-\right.$ 
$\left.\mathrm{CH}_{3}\right) \leftrightarrow 21.3\left(8^{\prime}-\mathrm{CH}_{3}\right), 1.08-1.16\left(\mathrm{~m}, 9^{\prime}-\mathrm{H}^{\mathrm{A}}\right) \leftrightarrow 44.7\left(\mathrm{C}-9^{\prime}\right), 1.08-1.16\left(\mathrm{~m}, 11^{\prime}-\mathrm{H}^{\mathrm{A}}\right) \leftrightarrow 30.2(\mathrm{C}-$ $\left.11^{\prime}\right), 1.21-1.34\left(\mathrm{~m}, 10^{\prime}-\mathrm{H}\right) \leftrightarrow 32.5\left(\mathrm{C}-10^{\prime}\right), 1.21-1.34\left(\mathrm{~m}, 9^{\prime}-\mathrm{H}^{\mathrm{B}}\right) \leftrightarrow 44.7\left(\mathrm{C}-9^{\prime}\right), 1.19-1.40(\mathrm{~m}$, $\left.11^{\prime}-\mathrm{H}^{\mathrm{B}}\right) \leftrightarrow 30.2\left(\mathrm{C}-11^{\prime}\right), 1.83\left(\mathrm{~d}, 6^{\prime}-\mathrm{CH}_{3}\right) \leftrightarrow 12.7\left(6^{\prime}-\mathrm{CH}_{3}\right), 2.64\left(\mathrm{~m}_{\mathrm{c}}, 8^{\prime}-\mathrm{H}\right), \leftrightarrow 31.0\left(\mathrm{C}-8^{\prime}\right), 3.05-$ $3.15\left(\mathrm{~m}, 5-\mathrm{CH}_{2}\right) \leftrightarrow 34.0\left(5-\mathrm{CH}_{2}\right), 3.79\left(\mathrm{~s}, 1 " '-\mathrm{OCH}_{3}\right) \leftrightarrow 55.5\left(1 '\right.$ '- $\left.\mathrm{OCH}_{3}\right), 3.79(\mathrm{~s}, 5$ ''-OCH 3$)$ $\leftrightarrow 55.5\left(5 " '-\mathrm{OCH}_{3}\right), 3.83\left(\mathrm{~m}_{\mathrm{c}}, 5-\mathrm{H}\right) \leftrightarrow 65.0(\mathrm{C}-5), 4.21\left(\mathrm{~d}, 2^{\prime \prime \prime}-\mathrm{C}-\mathrm{H}^{\mathrm{A}}\right) \leftrightarrow 38.4\left(2 " '-\mathrm{C}-\mathrm{H}_{2}\right), 5.00$ $\left(\mathrm{d}, 2^{\prime \prime \prime}-\mathrm{C}-\mathrm{H}^{\mathrm{B}}\right) \leftrightarrow 38.4\left(2^{\prime \prime}-\mathrm{C}-\mathrm{H}_{2}\right), 5.51\left(\mathrm{~d}, 7^{\prime}-\mathrm{H}\right) \leftrightarrow 147.3\left(\mathrm{C}-7^{\prime}\right), 6.42-6.45\left(\mathrm{~m}, 4\right.$ "'-H$^{\prime} \leftrightarrow 104.5$ (C-4"'), 6.42-6.45 (m, 6"'-H) ↔ 98.6 (C-6'"), 6.56 (d, 2'-H) ↔ 149.8 (C-2'), 6.64-6.68 (m, 2"H, 6"-H) 115.3 (C-2", C-6"), $6.71\left(\mathrm{dd}, 4^{\prime}-\mathrm{H}\right) \leftrightarrow 147.2\left(\mathrm{C}-4^{\prime}\right), 6.82\left(\mathrm{~d}, 5^{\prime}-\mathrm{H}\right) \leftrightarrow 115.2\left(\mathrm{C}-5^{\prime}\right)$, 6.92-6.97 (m, 3"-H, 5"-H) ↔ 130.8 (C-3", C-5"), 7.08-7.14 (m, 3"'-H) ↔ 131.5 (C-3"'), 7.37 $\left(\mathrm{dd}, 3^{\prime}-\mathrm{H}\right) \leftrightarrow 125.2\left(\mathrm{C}-3^{\prime}\right)$.

Specific rotation $(S, S, S)-34[a]_{D}^{20}=-688.3^{\circ}\left(\mathrm{c}=1.0\right.$ in $\left.\mathrm{CHCl}_{3}\right)$.

Specific rotation $(R, R, R)-34[a]_{D}^{20}=714.0^{\circ}\left(\mathrm{c}=1.0\right.$ in $\left.\mathrm{CHCl}_{3}\right)$.

HRMS (ESI) m/z: [M-H] ${ }^{+}$Calcd for $\mathrm{C}_{35} \mathrm{H}_{42} \mathrm{O}_{6} \mathrm{~N} 572.3018$; Found 572.3017

IR (KBr): $\tilde{v}=3340,2960,2925,2870,1675,1610,1590,1555,1510,1455,1360,1265,1245$, $1210,1160,1035,1000,935,885,825,750,610 \mathrm{~cm}^{-1}$. 
Christian Drescher, Morris Keller, Olivier Potterat, Matthias Hamburger and Reinhard Brückner, Structure-Elucidating Total Synthesis of the (Polyenoyl)tetramic Acid Militarinone C

(5S)-5-(1"'-Hydroxybenzyl)-1-(1'"',5'"-dimethoxybenzyl)-3-(1'Z,2'E,4'E,6'E, 8' R,10'R)-1'hydroxy-6',8',10'-trimethyldodeca-2',4',6'-trien-1'-ylidene)pyrrolidine-2,4-dion [Z-enol$(S, R, R)-34]$

in an 78:22 mixture with

(5S)-5-(1'-Hydroxybenzyl)-1-(1'"',5'"'-dimethoxybenzyl)-3-(1'E,2' $\left.E, 4^{\prime} E, 6{ }^{\prime} E, 8^{\prime} R, 10 ' R\right)-1$ 'hydroxy-6',8',10'-trimethyldodeca-2',4',6' -trien-1'-ylidene)pyrrolidine-2,4-dion [E-enol$(S, R, R)-34]$

and

(5R)-5-(1"-Hydroxybenzyl)-1-(1'"',5'"-dimethoxybenzyl)-3-(1'Z,2' $\left.E, 4^{\prime} E, 6{ }^{\prime} E, 8^{\prime} S, 10 ' S\right)-1^{\prime}$ hydroxy-6', 8',10'-trimethyldodeca-2',4',6'-trien-1'-ylidene)pyrrolidine-2,4-dion [Z-enol$(R, S, S)-34]$

in an 78:22 mixture with

(5R)-5-(1"'-Hydroxybenzyl)-1-(1'",5'"'-dimethoxybenzyl)-3-(1'E,2' $\left.E, 4^{\prime} E, 66^{\prime} E, 8^{\prime} S, 10 ' S\right)-1^{\prime}$ hydroxy-6',8',10'-trimethyldodeca-2',4',6' -trien-1'-ylidene)pyrrolidine-2,4-dion [E-enol$(R, S, S)-34]$
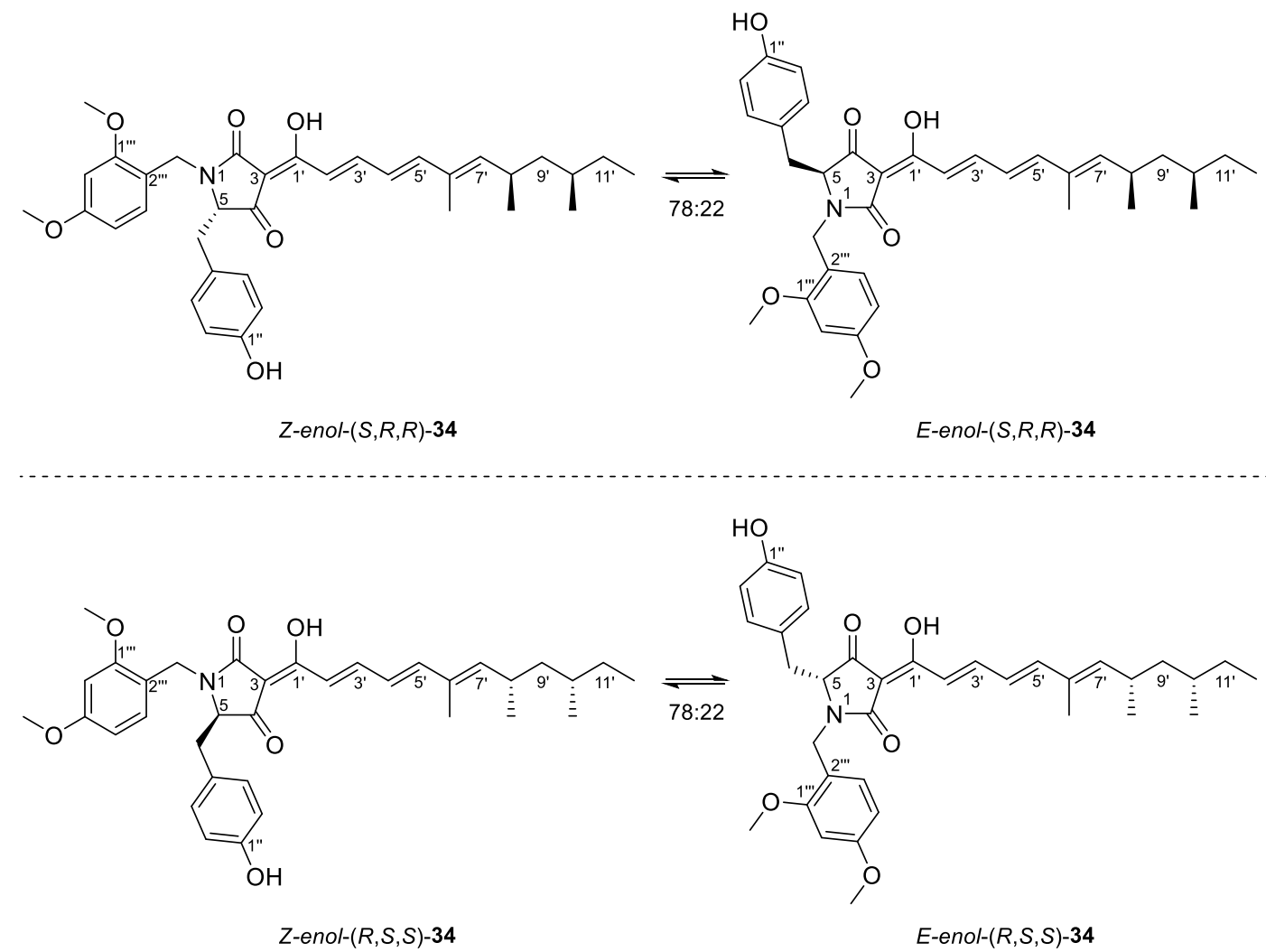
To a solution of the polyene $(S, R, R)-\mathbf{3 3}(67.4 \mathrm{mg}, 95.5 \mu \mathrm{mol})$ in THF $(0.8 \mathrm{~mL})$ was added TBAF ( $1 \mathrm{M}$ in THF, $338 \mu \mathrm{L}, 338 \mu \mathrm{mol}, 3.5$ eq.) at $25^{\circ} \mathrm{C}$. At this temperature the mixture was stirred for $1 \mathrm{~h}$. The solvent was removed under reduced pressure and the residue was obtained with $\mathrm{H}_{2} \mathrm{O}(2.5 \mathrm{~mL})$, aq. $\mathrm{H}_{2} \mathrm{SO}_{4}(4 \mathrm{M}, 2.5 \mathrm{~mL})$ and $\mathrm{Et}_{2} \mathrm{O}(12 \mathrm{~mL})$. The layers were separated and the organic layer was extracted with $\mathrm{Et}_{2} \mathrm{O}(3 \times 15 \mathrm{~mL})$. The combined organic layers were tried over $\mathrm{Na}_{2} \mathrm{SO}_{4}$ and concentrated under reduced pressure. The residue was purified by reverse phase flash chromatography $\left(\mathrm{MeCN}: \mathrm{H}_{2} \mathrm{O}: \mathrm{TFA}=85: 15: 0.1,1.5 \mathrm{~cm}, 10 \mathrm{~cm}, 2 \mathrm{~mL}\right)$. The tetramic acid $(S, R, R)-34(32.8 \mathrm{mg}, 61 \%, 58.6 \mu \mathrm{mol})$ was obtained as a yellow powder (mp.: $\left.78-80^{\circ} \mathrm{C}\right)$. It was assigned as a 78:22 mixture of the $Z$ - and the $E$-enol of the title compounds $(S, R, R)-34$. This ratio was determined by integrating dd's of the respective 3'-H [7.37 (E-enol) and 7.45 (Zenol)].

$\mathbf{R f}_{\mathbf{f}}\left(\mathrm{MeCN}: \mathrm{H}_{2} \mathrm{O}: \mathrm{TFA}=85: 15: 0.1\right)=0.18$

\section{Z-enol-(R,S,S)-34}

${ }^{1} \mathbf{H}$ NMR (500.10 MHz, $\mathrm{CDCl}_{3} / \mathrm{CHCl}_{3}$, DsBrDe12-410300, 13.12.19): $\delta=0.80-0.87$ (m, 3H, $\left.10 '-\mathrm{CH}_{3}\right), 0.80-0.87\left(\mathrm{~m}, 3 \mathrm{H}, 12^{\prime}-\mathrm{H}_{3}\right), 0.96\left(\mathrm{~d}, J_{8^{\prime}-\mathrm{CH}_{3}, 8^{\prime}}=6.6 \mathrm{~Hz}, 3 \mathrm{H}, 8^{\prime}-\mathrm{CH}_{3}\right), 1.08-1.16(\mathrm{~m}, 1 \mathrm{H}$, 9'- $\left.\mathrm{H}^{\mathrm{A}}\right), 1.08-1.16\left(\mathrm{~m}, 1 \mathrm{H}, 11^{\prime}-\mathrm{H}^{\mathrm{A}}\right), 1.21-1.34(\mathrm{~m}, 1 \mathrm{H}, 10 \mathrm{\prime}-\mathrm{H}), 1.21-1.34\left(\mathrm{~m}, 1 \mathrm{H}, 9^{\prime}-\mathrm{H}^{\mathrm{B}}\right), 1.21-$ $1.40\left(\mathrm{~m}, 1 \mathrm{H}, 11^{\prime}-\mathrm{H}^{\mathrm{B}}\right), 1.81\left(\mathrm{~s}, 3 \mathrm{H}, 66^{\prime}-\mathrm{CH}_{3}\right), 2.64\left(\mathrm{~m}_{\mathrm{c}}, 1 \mathrm{H}, 8^{\prime}-\mathrm{H}\right), 3.05-3.15\left(\mathrm{~m}, 2 \mathrm{H}, 5-\mathrm{CH}_{2}\right), 3.79$ (s, 3H, 1'"--OCH 3 ), 3.79 (s, 3H, 5'"--OCH $), 3.84\left(\mathrm{~m}_{\mathrm{c}}, 1 \mathrm{H}, 5-\mathrm{H}\right), 4.23$ (d, ${ }^{2} J=14.7 \mathrm{~Hz}, 1 \mathrm{H}, 2^{\prime \prime}-$ C-H $\left.\mathrm{H}^{\mathrm{A}}\right), 5.02\left(\mathrm{~d},{ }^{2} J=14.7 \mathrm{~Hz}, 1 \mathrm{H}, 2^{\prime \prime}-\mathrm{C}-\mathrm{H}^{\mathrm{B}}\right), 5.52\left(\mathrm{~d}, J_{7^{\prime}, 8^{\prime}}=9.7 \mathrm{~Hz}, 1 \mathrm{H}, 7^{\prime}-\mathrm{H}\right), 6.38$ (dd, $\left.J_{4^{\prime}, 3^{\prime}}=11.1 \mathrm{~Hz}, J_{4^{\prime}, 5^{\prime}}=14.8 \mathrm{~Hz}, 1 \mathrm{H}, 4^{\prime}-\mathrm{H}\right), 6.42-6.45$ (m, 2H, 4"'-H, 6"'-H), 6.64-6.68 (m, 1H, 5'H), 6.64-6.68 (m, 2H, 2"-H, 6"-H), 6.92-6.97 (m, 2H, 3"-H, 5"-H), 7.08-7.14 (m, 1H, 3"'-H), $7.11\left(\mathrm{~d}, J_{2^{\prime}, 3^{\prime}}=14.9 \mathrm{~Hz}, 1 \mathrm{H}, 2^{\prime}-\mathrm{H}\right), 7.45\left(\mathrm{dd}, J_{3^{\prime}, 4^{\prime}}=11.2 \mathrm{~Hz}, J_{3^{\prime}, 2^{\prime}}=15.1 \mathrm{~Hz}, 1 \mathrm{H}, 3^{\prime}-\mathrm{H}\right)$.

${ }^{13}$ C NMR (125.75 MHz, $\left.\mathrm{CDCl}_{3} / \mathrm{CHCl}_{3}, \mathrm{DsBrDe} 12-410304,13.12 .19\right): \delta=11.4$ (C-12'), 12.5 $\left(6 '-\mathrm{CH}_{3}\right), 19.2\left(10^{\prime}-\mathrm{CH}_{3}\right), 21.3\left(8^{\prime}-\mathrm{CH}_{3}\right), 30.1\left(\mathrm{C}-11{ }^{\prime}\right), 31.0\left(\mathrm{C}-8^{\prime}\right), 32.4(\mathrm{C}-10 '), 33.9\left(5-\mathrm{CH}_{2}\right)$, 38.4 (2'"-- $\left.\mathrm{CH}_{2}\right), 44.7$ (C-9'), 55.5 (1"'--OCH 3$), 55.5$ (5'"--OCH $), 65.1$ (C-5), 98.6 (C-6"'), 100.6 (C-3), 104.5 (C-4"'), 115.4 (C-2", C-6"), 116.2 (C-2"'), 120.1 (C-2'), 125.2 (C-4'), 126.9 (C-4"), 130.8 (C-3", C-5"), 131.5 (C-3"'), 132.9 (C-6'), 145.7 (C-3'), 147.4 (C-7'), 148.7 (C-5'), 154.9 (C-1"), 158.6 (C-1"'), 160.9 (C-5"'), 173.4 (C-1'), 173.9 (C-2), 194.9 (C-4).

edHSQC [,,short-range H,C-COSY“, $500.10 \mathrm{MHz} / 125.75 \mathrm{MHz}, \mathrm{CDCl}_{3} ; \delta\left({ }^{1} \mathrm{H}\right) \leftrightarrow \delta\left({ }^{13} \mathrm{C}\right)$ : 0.80-0.87 (m, 10'- $\left.\mathrm{CH}_{3}\right) \leftrightarrow 19.2\left(10^{\prime}-\mathrm{CH}_{3}\right), 0.80-0.87\left(\mathrm{~m}, 12^{\prime}-\mathrm{H}_{3}\right) \leftrightarrow 11.4\left(\mathrm{C}-12^{\prime}\right), 0.96\left(\mathrm{~d}, 8^{\prime}-\right.$ $\left.\mathrm{CH}_{3}\right) \leftrightarrow 21.3\left(8^{\prime}-\mathrm{CH}_{3}\right), 1.08-1.16\left(\mathrm{~m}, 9^{\prime}-\mathrm{H}^{\mathrm{A}}\right) \leftrightarrow 44.7\left(\mathrm{C}-9^{\prime}\right), 1.08-1.16\left(\mathrm{~m}, 11^{\prime}-\mathrm{H}^{\mathrm{A}}\right) \leftrightarrow 30.1(\mathrm{C}-$ 
11'), 1.21-1.34 (m, 10'-H) $32.4\left(\mathrm{C}-10^{\prime}\right), 1.21-1.34\left(\mathrm{~m}, 9^{\prime}-\mathrm{H}^{\mathrm{B}}\right) \leftrightarrow 44.7$ (C-9'), 1.21-1.40 (m, $\left.11^{\prime}-\mathrm{H}^{\mathrm{B}}\right) \leftrightarrow 30.1\left(\mathrm{C}-11^{\prime}\right), 1.81\left(\mathrm{~s}, 6^{\prime}-\mathrm{CH}_{3}\right) \leftrightarrow 12.5\left(6^{\prime}-\mathrm{CH}_{3}\right), 2.64\left(\mathrm{~m}_{\mathrm{c}}, 8^{\prime}-\mathrm{H}\right) \leftrightarrow 31.0\left(\mathrm{C}-8^{\prime}\right), 3.05-$ $3.15\left(\mathrm{~m}, 5-\mathrm{CH}_{2}\right) \leftrightarrow 34.0\left(5-\mathrm{CH}_{2}\right), 3.79\left(\mathrm{~s}, 1\right.$ '"-- $\left.\mathrm{OCH}_{3}\right) \leftrightarrow 55.5\left(1 " '-\mathrm{OCH}_{3}\right), 3.79(\mathrm{~s}, 5$ "'--OCH $)$ $\leftrightarrow 55.5\left(5^{\prime \prime \prime}-\mathrm{OCH}_{3}\right), 3.84\left(\mathrm{~m}_{\mathrm{c}}, 5-\mathrm{H}\right) \leftrightarrow 65.1(\mathrm{C}-5), 4.23\left(\mathrm{~d}, 2^{\prime \prime \prime}-\mathrm{C}-\mathrm{H}^{\mathrm{A}}\right) \leftrightarrow 38.4\left(2^{\prime \prime \prime}-\mathrm{CH}_{2}\right), 5.0(\mathrm{~d}$, 2"'-C-H $\left.{ }^{\mathrm{B}}\right) \leftrightarrow 38.4\left(2^{\prime \prime}-\mathrm{CH}_{2}\right), 5.52\left(\mathrm{~d}, 7^{\prime}-\mathrm{H}\right) \leftrightarrow 147.3\left(\mathrm{C}-7^{\prime}\right), 6.38\left(\mathrm{dd}, 4^{\prime}-\mathrm{H}\right) \leftrightarrow 125.2\left(\mathrm{C}-4^{\prime}\right)$, 6.42-6.45 (m, 4"'-H) 104.5 (C-4'"), 6.42-6.45 (m, 6"'-H), 98.6 (C-6"'), 6.64-6.68 (m, 5'-H)↔ 148.7 (C-5'), 6.64-6.68 (m, 2"-H, 6"-H) ↔ 115.3 (C-2", C-6"), 6.92-6.97 (m, 3"-H, 5"-H) ↔ 130.8 (C-3", C-5"), 7.08-7.14 (m, 3"'-H) ↔ 131.5 (C-3"'), 7.11 (d, 2'-H) ↔ 120.1 (C-2'), 7.45 $\left(\mathrm{dd}, 3^{\prime}-\mathrm{H}\right) \leftrightarrow 145.6\left(\mathrm{C}-3^{\prime}\right)$.

\section{E-enol-(R,S,S)-34:}

${ }^{1} \mathbf{H}$ NMR (500.10 MHz, $\mathrm{CDCl}_{3} / \mathrm{CHCl}_{3}$, DsBrDe12-410300, 13.12.19): 0.80-0.87 (m, 3H, 10'$\left.\mathrm{CH}_{3}\right), 0.80-0.87\left(\mathrm{~m}, 3 \mathrm{H}, 12^{\prime}-\mathrm{H}_{3}\right), 0.96\left(\mathrm{~d}, J_{8^{\prime}-\mathrm{CH}_{3}, 8^{\prime}}=6.7 \mathrm{~Hz}, 3 \mathrm{H}, 8^{\prime}-\mathrm{CH}_{3}\right), 1.08-1.16\left(\mathrm{~m}, 1 \mathrm{H}, 9^{\prime}-\right.$ $\left.\mathrm{H}^{\mathrm{A}}\right), 1.08-1.16\left(\mathrm{~m}, 1 \mathrm{H}, 11^{\prime}-\mathrm{H}^{\mathrm{A}}\right), 1.21-1.34\left(\mathrm{~m}, 1 \mathrm{H}, 10^{\prime}-\mathrm{H}\right), 1.21-1.34\left(\mathrm{~m}, 1 \mathrm{H}, 9^{\prime}-\mathrm{H}^{\mathrm{B}}\right), 1.19-1.40$ $\left(\mathrm{m}, 1 \mathrm{H}, 11^{\prime}-\mathrm{H}^{\mathrm{B}}\right), 1.83\left(\mathrm{~s}, 6^{\prime}-\mathrm{CH}_{3}\right), 2.64\left(\mathrm{~m}_{\mathrm{c}}, 1 \mathrm{H}, 8^{\prime}-\mathrm{H}\right), 3.05-3.15\left(\mathrm{~m}, 2 \mathrm{H}, 5-\mathrm{CH}_{2}\right), 3.79(\mathrm{~s}, 3 \mathrm{H}$, 1'"- $\left.-\mathrm{OCH}_{3}\right), 3.79$ (s, 3H, 5"'-OCH 3 ), 3.99 (t, $\left.J_{5,5-\mathrm{CH}_{2}}=4.4 \mathrm{~Hz}, 1 \mathrm{H}, 5-\mathrm{H}\right), 4.25\left(\mathrm{~d},{ }^{2} J=14.7 \mathrm{~Hz}\right.$, $\left.1 \mathrm{H}, 2^{\prime \prime}-\mathrm{C}-\mathrm{H}^{\mathrm{A}}\right), 5.09\left(\mathrm{~d},{ }^{2} J=14.7 \mathrm{~Hz}, 1 \mathrm{H}, 2^{2}{ }^{\prime}-\mathrm{C}^{-\mathrm{H}^{\mathrm{B}}}\right), 5.52\left(\mathrm{~d}, J_{7^{\prime}, 8^{\prime}}=9.9 \mathrm{~Hz}, 1 \mathrm{H}, 7^{\prime}-\mathrm{H}\right), 6.42-6.45$ (m, 1H, 4"'-H), 6.42-6.45 (m, 1H, 6"'-H), 6.56 (d, $\left.J_{2^{\prime}, 3^{\prime}}=15.0 \mathrm{~Hz}, 1 \mathrm{H}, 2^{\prime}-\mathrm{H}\right), 6.64-6.68$ (m, 2H, 2"-H, 6"-H), 6.71 (dd, $\left.J_{4^{\prime}, 3^{\prime}}=J_{4^{\prime}, 5^{\prime}}=11.4 \mathrm{~Hz}, 1 \mathrm{H}, 4^{\prime}-\mathrm{H}\right), 6.82$ (d, $\left.J_{5^{\prime}, 4^{\prime}}=11.7 \mathrm{~Hz}, 1 \mathrm{H}, 5^{\prime}-\mathrm{H}\right), 6.92-$ 6.97 (m, 2H, 3"-H, 5"-H), 7.08-7.14 (m, 1H, 3"'-H), 7.37 (dd, J J', 3 $^{\prime}=11.8 \mathrm{~Hz}, J_{3^{\prime}, 2^{\prime}}=15.0 \mathrm{~Hz}$, $\left.1 \mathrm{H}, 3^{\prime}-\mathrm{H}\right)$.

${ }^{13} \mathrm{C}$ NMR (125.75 MHz, $\left.\mathrm{CDCl}_{3} / \mathrm{CHCl}_{3}, \mathrm{DsBrDe} 12-410304,13.12 .19\right): \delta=11.4(\mathrm{C}-12$ '), 12.64 $\left(6^{\prime}-\mathrm{CH}_{3}\right), 19.1\left(10^{\prime}-\mathrm{CH}_{3}\right), 21.3\left(8^{\prime}-\mathrm{CH}_{3}\right), 30.2\left(\mathrm{C}-11^{\prime}\right), 31.0\left(\mathrm{C}-8^{\prime}\right), 32.4(\mathrm{C}-10 '), 34.0\left(5-\mathrm{CH}_{2}\right)$, 38.4 (2'"-- $\left.\mathrm{CH}_{2}\right), 44.7$ (C-9'), 55.5 (1"'--OCH 3$), 55.5$ (5'"--OCH $), 65.1$ (C-5), 98.5 (C-6"'), 101.1 (C-3), 104.6 (C-4"'), 115.3 (C-2", C-6"), 115.8 (C-5'), 116.2 (C-2'"), 125.2 (C-3'), 127.0 (C-4"), 130.8 (C-3", C-5"), 131.5 (C-3"'), 133.0 (C-6'), 147.3 (C-7'), 147.5 (C-4'), 149.9 (C-2'), 154.8 (C-1"), 158.6 (C-1"'), 160.7 (C-5"'), 173.2 (C-1'), 173.8 (C-2), 194.4 (C-4).

edHSQC [,,short-range H,C-COSY“, $500.10 \mathrm{MHz} / 125.75 \mathrm{MHz}, \mathrm{CDCl}_{3} ; \delta\left({ }^{1} \mathrm{H}\right) \leftrightarrow \delta\left({ }^{13} \mathrm{C}\right)$ : 0.80-0.87 (m, 10'- $\left.\mathrm{CH}_{3}\right) \leftrightarrow 19.1\left(10^{\prime}-\mathrm{CH}_{3}\right), 0.80-0.87\left(\mathrm{~m}, 12^{\prime}-\mathrm{H}_{3}\right) \leftrightarrow 11.4(\mathrm{C}-12 '), 0.96$ (d, 8'$\left.\mathrm{CH}_{3}\right) \leftrightarrow 21.3\left(8^{\prime}-\mathrm{CH}_{3}\right), 1.08-1.16\left(\mathrm{~m}, 9^{\prime}-\mathrm{H}^{\mathrm{A}}\right) \leftrightarrow 44.7\left(\mathrm{C}-9^{\prime}\right), 1.08-1.16\left(\mathrm{~m}, 11^{\prime}-\mathrm{H}^{\mathrm{A}}\right) \leftrightarrow 30.2(\mathrm{C}-$ $\left.11^{\prime}\right), 1.21-1.34\left(\mathrm{~m}, 10^{\prime}-\mathrm{H}\right) \leftrightarrow 32.4(\mathrm{C}-10 '), 1.21-1.34\left(\mathrm{~m}, 9^{\prime}-\mathrm{H}^{\mathrm{B}}\right) \leftrightarrow 44.7\left(\mathrm{C}-9^{\prime}\right), 1.19-1.40(\mathrm{~m}$, $\left.11^{\prime}-\mathrm{H}^{\mathrm{B}}\right) \leftrightarrow 30.2\left(\mathrm{C}-11^{\prime}\right), 1.83\left(\mathrm{~s}, 6^{\prime}-\mathrm{CH}_{3}\right) \leftrightarrow 12.6\left(6^{\prime}-\mathrm{CH}_{3}\right), 2.64\left(\mathrm{~m}_{\mathrm{c}}, 8^{\prime}-\mathrm{H}\right) \leftrightarrow 31.0\left(\mathrm{C}-8^{\prime}\right), 3.05-$ $3.15\left(\mathrm{~m}, 5-\mathrm{CH}_{2}\right) \leftrightarrow 34.0\left(5-\mathrm{CH}_{2}\right), 3.79\left(\mathrm{~s}, 1\right.$ '"'- $\left.\mathrm{OCH}_{3}\right) \leftrightarrow 55.5\left(1 '\right.$ '- $\left.-\mathrm{OCH}_{3}\right), 3.79\left(\mathrm{~s}, 5\right.$ '"- $\left.-\mathrm{OCH}_{3}\right)$ 
$\leftrightarrow 55.5\left(5 ' "-\mathrm{OCH}_{3}\right), 3.99(\mathrm{t}, 5-\mathrm{H}) \leftrightarrow 65.1(\mathrm{C}-5), 4.25\left(\mathrm{~d}, 2\right.$ '"-C-H $\left.{ }^{\mathrm{A}}\right) \leftrightarrow 38.4\left(2^{\prime \prime}-\mathrm{CH}_{2}\right), 5.09(\mathrm{~d}$, 2"'-C-H $\left.{ }^{\mathrm{B}}\right) \leftrightarrow 38.4\left(2^{\prime \prime}-\mathrm{CH}_{2}\right), 5.52\left(\mathrm{~d}, 7^{\prime}-\mathrm{H}\right) \leftrightarrow 147.3(\mathrm{C}-7$ '), 6.42-6.45 (m, 4"'-H) 4'"), 6.42-6.45 (m, 6'"-H) 98.5 (C-6"'), 6.56 (d, 2'-H) 149.9 (C-2'), 6.64-6.68 (m, 2"-H, 6"$\mathrm{H}) \leftrightarrow 115.3$ (C-2", C-6"), 6.71 (dd, 4'-H) 147.5 (C-4'), 6.82 (d, 5'-H) 115.8 (C-5'), 6.926.97 (m, 3"-H, 5"-H) ↔ 130.8 (C-3", C-5"), 7.08-7.14 (m, 3'"-H) 131.5 (C-3"'), 7.37 (dd, 3'$\mathrm{H}) \leftrightarrow 125.2\left(\mathrm{C}-3^{\prime}\right)$.

Specific rotation $(R, S, S)-34[a]_{D}^{20}=650.1^{\circ}\left(\mathrm{c}=1.0\right.$ in $\left.\mathrm{CHCl}_{3}\right)$.

Specific rotation $(S, R, R)-34[a]_{D}^{20}=-727.0^{\circ}\left(\mathrm{c}=1.0\right.$ in $\left.\mathrm{CHCl}_{3}\right)$.

HRMS (ESI) m/z: $[\mathrm{M}+\mathrm{H}]^{+}$Calcd for $\mathrm{C}_{35} \mathrm{H}_{44} \mathrm{O}_{6} \mathrm{~N}$ 574.3163; Found 574.3160

IR (KBr): $\tilde{v}=3340,2960,2925,2870,1675,1610,1590,1555,1510,1455,1360,1265,1245$, $1210,1160,1035,1000,935,915,885,825,750,610 \mathrm{~cm}^{-1}$. 
(5S)-5-(1'"-Hydroxybenzyl)-3-(1'Z,2' $E, 4^{\prime} E, 6$ ' $\left.E, 8^{\prime} S, 10 ' S\right)-1$ '-hydroxy-6',8',10'trimethyldodeca-2',4',6'-trien-1'-ylidene)pyrrolidine-2,4-dion [Z-enol-(S,S,S)-1] in an 75:25 mixture with

(5S)-5-(1'"-Hydroxybenzyl)-3-(1'E,2' $\left.E, 4^{\prime} E, 6^{\prime} E, 8^{\prime} S, 10 ' S\right)-1^{\prime}-h y d r o x y-6 ', 8 ', 10 '-$ trimethyldodeca-2',4',6'-trien-1'-ylidene)pyrrolidine-2,4-dion [E-enol-(S,S,S)-1] and (5R)-5-(1''-Hydroxybenzyl)-3-(1'Z,2' $\left.E, 4^{\prime} E, 6^{\prime} E, 8^{\prime} R, 10^{\prime} R\right)-1^{\prime}-h y d r o x y-6 ', 8^{\prime}, 10 '-$ trimethyldodeca-2',4',6'-trien-1'-ylidene)pyrrolidine-2,4-dion [Z-enol-(R,R,R)-1] in an 75:25 mixture with

(5R)-5-(1'-Hydroxybenzyl)-3-(1'E,2' $\left.E, 4^{\prime} E, 6^{\prime} E, 8^{\prime} R, 10 ' R\right)-1^{\prime}-h y d r o x y-6 ', 8^{\prime}, 10^{\prime}-$ trimethyldodeca-2',4',6'-trien-1'-ylidene)pyrrolidine-2,4-dion [E-enol-(R,R,R)-1]
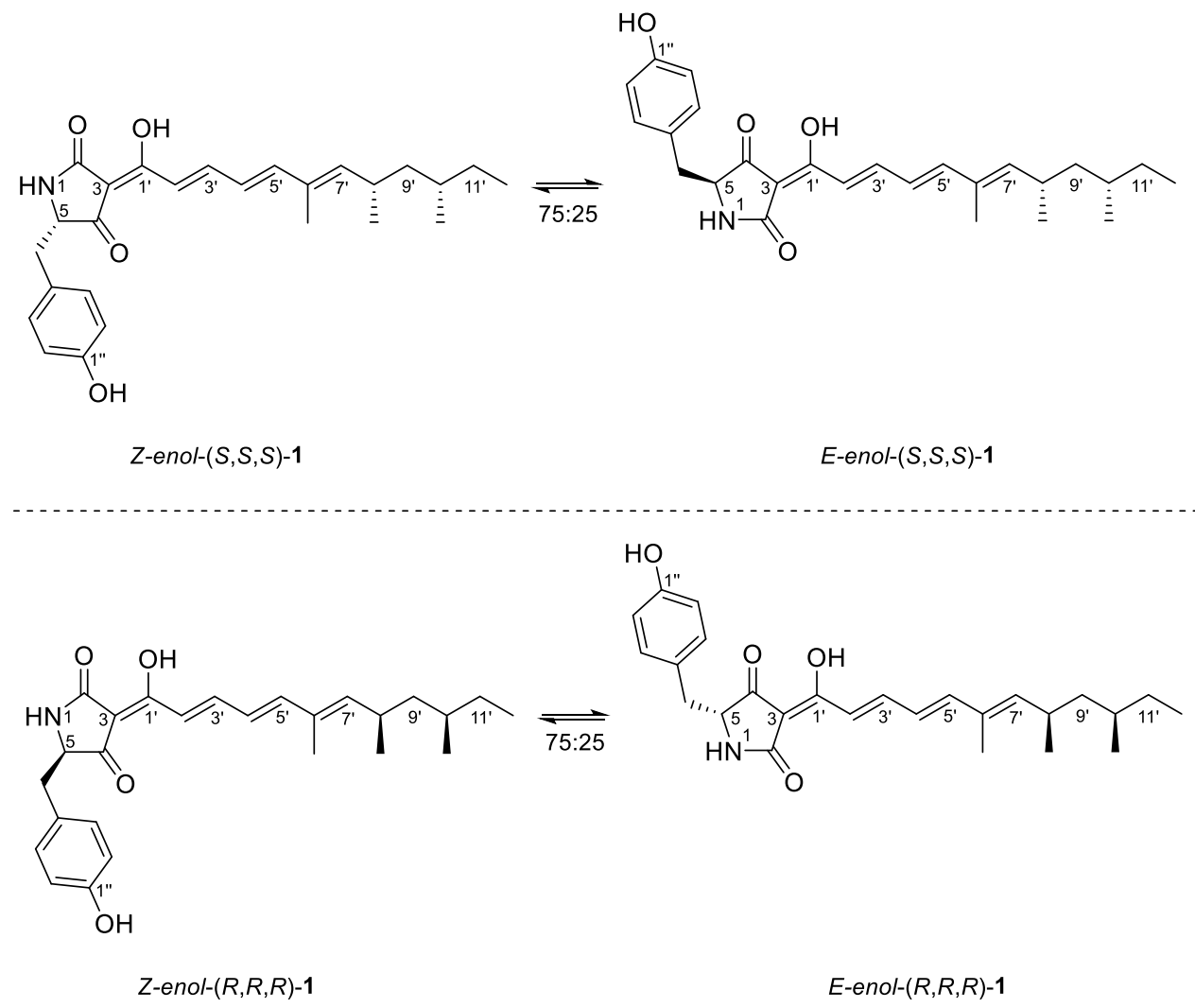

The DMB-tetramic acid $(S, S, S)-34(20.0 \mathrm{mg}, 35.0 \mu \mathrm{mol})$ has been dissolved in $\mathrm{CH}_{2} \mathrm{Cl}_{2}$ (2.0 mL). First Thioanisole ( $200 \mu \mathrm{L}, 210 \mathrm{mg}, 1.75 \mathrm{mmol}, 50$ eq.) and then $\mathrm{F}_{3} \mathrm{CCO}_{2} \mathrm{H}(0.5 \mathrm{~mL})$ was added at $0^{\circ} \mathrm{C}$. Die temperature was warmed up to $25^{\circ} \mathrm{C}$ and held this temperature for $1 \mathrm{~h}$. The mixture was diluted with $\mathrm{H}_{2} \mathrm{O}(2 \mathrm{~mL})$. The layers were separated and the aq. layer was 
extracted with $\mathrm{CH}_{2} \mathrm{Cl}_{2}(3 \times 5 \mathrm{~mL})$. The combined organic layers were washed with brine $(5 \mathrm{~mL})$ and tried over $\mathrm{Na}_{2} \mathrm{SO}_{4}$. The solvent was removed under reduced pressure and to the residue was added $\mathrm{MeOH}(3 \mathrm{~mL})$. The resulting suspension was filtered off and the filtrate was concentrated under reduced pressure. The residue was purified by reverse phase flash chromatography (MeCN: $\mathrm{H}_{2} \mathrm{O}: \mathrm{TFA}=75: 25: 0.1,1.5 \mathrm{~cm}, 10 \mathrm{~cm}, 2 \mathrm{~mL}$ ). The solvent was removed under reduced pressure and the residue was purified by preparative HPLC [Varian ProStar, MeCN: $\mathrm{H}_{2} \mathrm{O}$ :TFA $=85: 15: 0.1,20 \mathrm{~mL} / \mathrm{min}$, injections of $3 \mathrm{mg}$ of crude product per run, $20^{\circ} \mathrm{C}, \lambda_{\mathrm{UV} / \mathrm{vis} \text {-detector }}=387$ $\mathrm{nm}$; $\left.\mathrm{t}_{\text {retention, }(S, S, S)-\mathbf{1}}=11.7 \mathrm{~min}\right]$. The $(S, S, S)$-militarinone $\mathrm{C}(S, S, S)-\mathbf{1}(11.4 \mathrm{mg}, 77 \%, 27.0 \mu \mathrm{mol})$ was obtained as a yellow powder $\left(\mathrm{mp} .: 7^{\circ} \mathrm{C}\right)$. It was assigned as a 75:25 mixture of the $Z$ - and the $E$-enol of the title compounds $(S, S, S)$-1. This ratio was determined by integrating dd's of the respective 3 '-H $[7.40$ (E-enol) and 7.55 (Z-enol)].

The enantiomer $(R, R, R)-\mathbf{1}$ was obtained analogously in $75 \%$ yield.

$\mathbf{R}_{\mathbf{f}}\left(\mathrm{MeCN}: \mathrm{H}_{2} \mathrm{O}: \mathrm{TFA}=75: 25: 0.1\right)=0.16$

${ }^{1} \mathbf{H}$ NMR $(500.10 \mathrm{MHz}, \mathrm{MeOD} / \mathrm{MeOH}$, sample contained 1 weight-\% of MeCN, trace amounts of $\mathrm{CH}_{2} \mathrm{Cl}_{2}$ and unknown impurity s at 3.63, DsBrSe29-5040, 2.10.2017): 0.84-0.88 (m, 3H, 10'$\left.\mathrm{CH}_{3}\right), 0.80-0.87\left(\mathrm{~m}, 3 \mathrm{H}, 12^{\prime}-\mathrm{H}_{3}\right), 0.98\left(\mathrm{~d}, J_{8^{\prime}-\mathrm{CH}_{3}, 8^{\prime}}=6.6 \mathrm{~Hz}, 3 \mathrm{H}, 8^{\prime}-\mathrm{CH}_{3}\right), 1.10-1.19$ (m, 1H, 9'$\left.\mathrm{H}^{\mathrm{A}}\right), 1.10-1.19\left(\mathrm{~m}, 1 \mathrm{H}, 11^{\prime}-\mathrm{H}^{\mathrm{A}}\right), 1.20-1.37\left(\mathrm{~m}, 1 \mathrm{H}, 10^{\prime}-\mathrm{H}\right), 1.20-1.37\left(\mathrm{~m}, 1 \mathrm{H}, 9^{\prime}-\mathrm{H}^{\mathrm{B}}\right), 1.20-1.37$ $\left(\mathrm{m}, 1 \mathrm{H}, 11^{\prime}-\mathrm{H}^{\mathrm{B}}\right), 1.85\left(\mathrm{~d},{ }^{4} J_{6}-\mathrm{CH}_{3}, 7^{\prime}=0.9 \mathrm{~Hz}, 3 \mathrm{H}, 66^{\prime}-\mathrm{CH}_{3}\right), 2.70\left(\mathrm{~m}_{\mathrm{c}}, 1 \mathrm{H}, 8^{\prime}-\mathrm{H}\right)$, AB signal $\left(\delta_{\mathrm{A}}=2.87, \delta_{\mathrm{B}}=3.98, J_{\mathrm{AB}}=14.1 \mathrm{~Hz}\right.$, in addition split by $J_{5-\mathrm{CH}^{\mathrm{A}}, 5}=4.4 \mathrm{~Hz}, J_{5-\mathrm{CH}^{\mathrm{B}}, 5}=6.0 \mathrm{~Hz}, 2 \mathrm{H}$, 5- $\mathrm{CH}_{2}$ ), 4.06 (br. s, 1H, 5-H), 5.59 (d, $\left.J_{7^{\prime}, 8^{\prime}}=9.8 \mathrm{~Hz}, 1 \mathrm{H}, 7^{\prime}-\mathrm{H}\right), 6.45$ (dd, $J_{4^{\prime}, 3^{\prime}}=11.2 \mathrm{~Hz}$, $\left.J_{4^{\prime}, 5^{\prime}}=15.1 \mathrm{~Hz}, 1 \mathrm{H}, 4^{\prime}-\mathrm{H}\right), 6.64-6.68\left(\mathrm{~m}, 2 \mathrm{H}, 2^{\prime \prime}-\mathrm{H}, 66^{\prime \prime}-\mathrm{H}\right), 6.77$ (d, $\left.J_{5^{\prime}, 4^{\prime}}=15.2 \mathrm{~Hz}, 1 \mathrm{H}, 5^{\prime}-\mathrm{H}\right)$, 6.96-6.99 (m, 2H, 3"-H, 5"-H), $7.10\left(\mathrm{~d}, J_{2^{\prime}, 3^{\prime}}=15.2 \mathrm{~Hz}, 1 \mathrm{H}, 2^{\prime}-\mathrm{H}\right), 7.54\left(\mathrm{dd}, J_{3^{\prime}, 4^{\prime}}=11.4 \mathrm{~Hz}\right.$, $\left.J_{3^{\prime}, 2^{\prime}}=15.3 \mathrm{~Hz}, 1 \mathrm{H}, 3^{\prime}-\mathrm{H}\right)$.

${ }^{13}$ C NMR (125.75 MHz, MeOD/MeOH, DsBrSe29-5044, 2.10.2017): $\delta=11.7$ (C-12'), 12.6 (6'- $\left.\mathrm{CH}_{3}\right), 19.5\left(10^{\prime}-\mathrm{CH}_{3}\right), 21.5\left(8^{\prime}-\mathrm{CH}_{3}\right), 31.2\left(\mathrm{C}-11^{\prime}\right), 32.1\left(\mathrm{C}-8^{\prime}\right), 33.7(\mathrm{C}-10 '), 37.6\left(5-\mathrm{CH}_{2}\right)$, 45.8 (C-9'), 64.4 (C-5), 116.1 (C-2", C-6"), 120.7 (C-2'), 126.3 (C-4'), 127.7 (C-4"), 131.8 (C3", C-5"), 134.3 (C-6'), 147.4 (C-3'), 148.4 (C-7'), 150.4 (C-5'), 157.3 (C-1"), 175.3 (C-2).*

*C-3, C-4 and C-1' were not found.

edHSQC [,,short-range H,C-COSY“, 500.10 MHz/125.75 MHz, MeOD; $\delta\left({ }^{1} \mathrm{H}\right) \leftrightarrow \delta\left({ }^{13} \mathrm{C}\right)$ : 0.84-0.88 (m, 10'- $\left.\mathrm{CH}_{3}\right) \leftrightarrow 19.5\left(10^{\prime}-\mathrm{CH}_{3}\right), 0.80-0.87\left(\mathrm{~m}, 12^{\prime}-\mathrm{H}_{3}\right) \leftrightarrow 11.7\left(\mathrm{C}-12^{\prime}\right), 0.98$ (d, 8'$\left.\mathrm{CH}_{3}\right) \leftrightarrow 21.5\left(8^{\prime}-\mathrm{CH}_{3}\right), 1.10-1.19\left(\mathrm{~m}, 9^{\prime}-\mathrm{H}^{\mathrm{A}}\right) \leftrightarrow 45.8\left(\mathrm{C}-9^{\prime}\right), 1.10-1.19\left(\mathrm{~m}, 11^{\prime}-\mathrm{H}^{\mathrm{A}}\right) \leftrightarrow 31.2(\mathrm{C}-$ 
$\left.11^{\prime}\right), 1.20-1.37\left(\mathrm{~m}, 10^{\prime}-\mathrm{H}\right) \leftrightarrow 33.7\left(\mathrm{C}-10^{\prime}\right), 1.20-1.37\left(\mathrm{~m}, 9^{\prime}-\mathrm{H}^{\mathrm{B}}\right) \leftrightarrow 45.8\left(\mathrm{C}-9^{\prime}\right), 1.20-1.37(\mathrm{~m}$, $\left.11^{\prime}-\mathrm{H}^{\mathrm{B}}\right) \leftrightarrow 31.2\left(\mathrm{C}-11^{\prime}\right), 1.85\left(\mathrm{~d}, 6^{\prime}-\mathrm{CH}_{3}\right) \leftrightarrow 12.6\left(6^{\prime}-\mathrm{CH}_{3}\right), 2.70\left(\mathrm{~m}_{\mathrm{c}}, 8^{\prime}-\mathrm{H}\right) \leftrightarrow 32.1\left(\mathrm{C}-8^{\prime}\right), \mathrm{AB}$ signal $\left(\delta_{\mathrm{A}}=2.87, \delta_{\mathrm{B}}=3.98,5-\mathrm{CH}_{2}\right) \leftrightarrow 37.6\left(5-\mathrm{CH}_{2}\right), 4.06($ br. s, 5-H) $\leftrightarrow 64.3(\mathrm{C}-5), 5.59(\mathrm{~d}$, 7'-H) 148.4 (C-7), 6.45 (dd, 4'-H) 126.3 (C-4'), 6.64-6.68 (m, 2"-H, 6"-H) 116.1 (C2", C-6"), 6.77 (d, 5'-H) ↔ 150.3 (C-5'), 6.96-6.99 (m, 3"-H, 5"-H) ↔ 131.8 (C-3", C-5"), 7.10 $\left(\mathrm{d}, 2^{\prime}-\mathrm{H}\right) \leftrightarrow 120.7\left(\mathrm{C}-2^{\prime}\right), 7.54\left(\mathrm{dd}, 3^{\prime}-\mathrm{H}\right) \leftrightarrow 147.4\left(\mathrm{C}-3^{\prime}\right)$.

Specific rotation $(S, S, S)-\mathbf{1}[a]_{D}^{20}=-350.0^{\circ}(\mathrm{c}=0.17$ in $\mathrm{MeOH})$.

Specific rotation $(R, R, R)-1[a]_{D}^{20}=353.0^{\circ}(\mathrm{c}=0.17$ in $\mathrm{MeOH})$.

HRMS (ESI) m/z: [M+H] $]^{+}$Calcd for $\mathrm{C}_{26} \mathrm{H}_{34} \mathrm{O}_{4} \mathrm{~N}$ 424.2482; Found 424.2482

IR (KBr): $\tilde{v}=3285,3060,3035,2960,2925,2870,2855,1650,1625,1595,1550,1515,1430$, $1375,1310,1280,1230,1205,1175,1030,1000,955,895,815,610 \mathrm{~cm}^{-1}$. 
(5S)-5-(1''-Hydroxybenzyl)-3-(1'Z,2' $\left.E, 4^{\prime} E, 6^{\prime} E, 8^{\prime} R, 10 ' R\right)-1$ '-hydroxy-6',8',10'trimethyldodeca-2',4',6'-trien-1'-ylidene)pyrrolidine-2,4-dion [Z-enol-(S,R,R)-1] ${ }^{17}$ in an 81:19 mixture with

(5S)-5-(1'-Hydroxybenzyl)-3-(1'E,2' $\left.E, 4^{\prime} E, 6^{\prime} E, 8^{\prime} R, 10 ' R\right)-1^{\prime}-h y d r o x y-6 ', 8^{\prime}, 10^{\prime}-$ trimethyldodeca-2',4',6'-trien-1'-ylidene)pyrrolidine-2,4-dion [E-enol-(S,R,R)-1] and (5R)-5-(1'-Hydroxybenzyl)-3-(1'Z,2' $E, 4^{\prime} E, 6$ ' $\left.E, 8^{\prime} S, 10 ' S\right)-1$ '-hydroxy-6',8',10'trimethyldodeca-2',4',6'-trien-1'-ylidene)pyrrolidine-2,4-dion [Z-enol-(R,S,S)-1] in an 81:19 mixture with

(5R)-5-(1'-Hydroxybenzyl)-3-(1'E,2' $\left.E, 4^{\prime} E, 6^{\prime} E, 8^{\prime} R, 10 ' R\right)-1^{\prime}-h y d r o x y-6 ', 8^{\prime}, 10^{\prime}-$ trimethyldodeca-2',4',6'-trien-1'-ylidene)pyrrolidine-2,4-dion [E-enol-(R,S,S)-1]

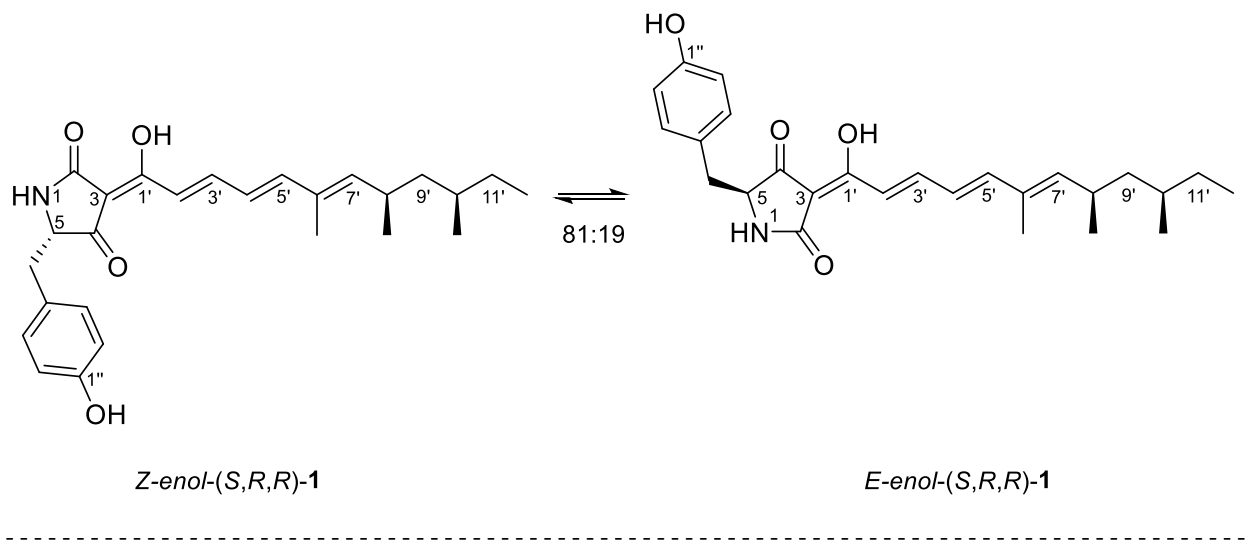

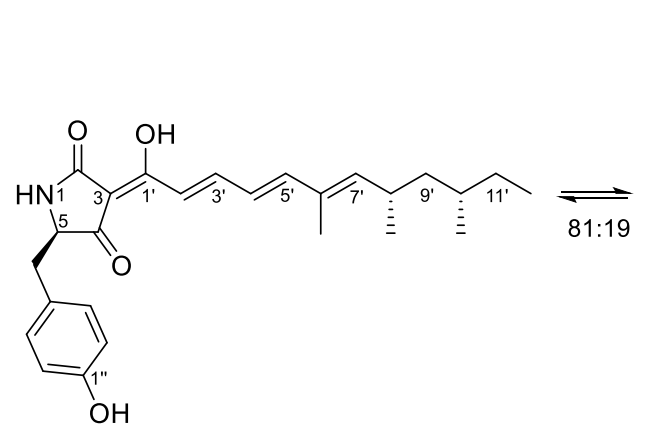

Z-enol-(R,S,S)-1

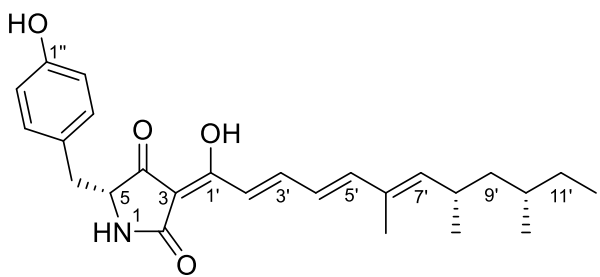

E-enol-(R,S,S)-1

The DMB-tetramic acid $(S, R, R)-34(35.0 \mathrm{mg}, 61.2 \mu \mathrm{mol})$ has been dissolved in $\mathrm{CH}_{2} \mathrm{Cl}_{2}$ (3.5 mL). First Thioansisole $\left(350 \mu \mathrm{L}, 368 \mathrm{mg}, 3.06 \mathrm{mmol}, 50\right.$ eq.) and then $\mathrm{F}_{3} \mathrm{CCO}_{2} \mathrm{H}(0.9 \mathrm{~mL})$

\footnotetext{
${ }^{17}$ K. Schmidt, U. Riese, Z. Li, M. Hamburger, J. Nat. Prod. 2003, 66, 378-383.
} 
was added at $0^{\circ} \mathrm{C}$. Die temperature was warmed up to $25^{\circ} \mathrm{C}$ and held this temperature for $1 \mathrm{~h}$. The mixture was diluted with $\mathrm{H}_{2} \mathrm{O}(2 \mathrm{~mL})$. The layers were separated and the aq. layer was extracted with $\mathrm{CH}_{2} \mathrm{Cl}_{2}(3 \times 15 \mathrm{~mL})$. Die combined organic layers were washed with brine $(10 \mathrm{~mL})$ and tried over $\mathrm{Na}_{2} \mathrm{SO}_{4}$. The solvent was removed under reduced pressure and to the residue was added $\mathrm{MeOH}(5 \mathrm{~mL})$. The resulting suspension was filtered off and the filtrate was concentrated under reduced pressure. The residue was purified by reverse phase flash chromatography (MeCN:H $\left.\mathrm{H}_{2} \mathrm{O}: \mathrm{TFA}=75: 25: 0.1,1.5 \mathrm{~cm}, 10 \mathrm{~cm}, 2 \mathrm{~mL}\right)$. The solvent was removed under reduced pressure and the residue was purified by preparative HPLC [Varian ProStar, $\mathrm{MeCN}: \mathrm{H}_{2} \mathrm{O}: \mathrm{TFA}=85: 15: 0.1,20 \mathrm{~mL} / \mathrm{min}$, injections of $3 \mathrm{mg}$ of crude product per run, $\left.20^{\circ} \mathrm{C}, \lambda_{\mathrm{UV} / \text { vis-detector }}=387 \mathrm{~nm} ; \mathrm{t}_{\text {retention, }(S, R, R)-\mathbf{1}}=11.7 \mathrm{~min}\right]$. The $(S, R, R)$-militarinone $\mathrm{C}(S, R, R)-\mathbf{1}$ $(19.4 \mathrm{mg}, 75 \%, 46.1 \mu \mathrm{mol})$ was obtained as a yellow powder $\left(\mathrm{mp} .: 75^{\circ} \mathrm{C}\right)$. It was assigned as a 81:19 mixture of the $Z$ - and the $E$-enol of the title compounds $(S, R, R)-\mathbf{1}$. This ratio was determined by integrating dd's of the respective 3'-H [7.40 (E-enol) and 7.55 (Z-enol)].

The enantiomer $(R, S, S)-\mathbf{1}$ was obtained analogously in $76 \%$ yield.

$\mathbf{R}_{\mathbf{f}}\left(\mathrm{MeCN}: \mathrm{H}_{2} \mathrm{O}: \mathrm{TFA}=75: 25: 0.1\right)=0.16$

${ }^{1} \mathbf{H}$ NMR (500.10 MHz, MeOD/MeOH, DsBrSe25-201100, 26.9.2019): 0.84-0.88 (m, 3H, 10'$\left.\mathrm{CH}_{3}\right), 0.80-0.87\left(\mathrm{~m}, 3 \mathrm{H}, 12^{\prime}-\mathrm{H}_{3}\right), 0.98\left(\mathrm{~d}, J_{8^{\prime}-\mathrm{CH}_{3}, 8^{\prime}}=6.6 \mathrm{~Hz}, 3 \mathrm{H}, 8^{\prime}-\mathrm{CH}_{3}\right), 1.10-1.19\left(\mathrm{~m}, 1 \mathrm{H}, 9^{\prime}-\right.$ $\left.\mathrm{H}^{\mathrm{A}}\right), 1.10-1.19\left(\mathrm{~m}, 1 \mathrm{H}, 11^{\prime}-\mathrm{H}^{\mathrm{A}}\right), 1.22-1.38\left(\mathrm{~m}, 1 \mathrm{H}, 10^{\prime}-\mathrm{H}\right), 1.22-1.38\left(\mathrm{~m}, 1 \mathrm{H}, 9^{\prime}-\mathrm{H}^{\mathrm{B}}\right), 1.22-1.38$ $\left(\mathrm{m}, 1 \mathrm{H}, 11^{\prime}-\mathrm{H}^{\mathrm{B}}\right), 1.85\left(\mathrm{~d}, J_{6^{\prime}-\mathrm{CH}_{3}, 7^{\prime}}=1.0 \mathrm{~Hz}, 3 \mathrm{H}, 66^{\prime}-\mathrm{CH}_{3}\right), 2.70\left(\mathrm{~m}_{\mathrm{c}}, 1 \mathrm{H}, 8^{\prime}-\mathrm{H}\right), \mathrm{AB}$ signal $\left(\delta_{\mathrm{A}}=2.87, \delta_{\mathrm{B}}=3.98, J_{\mathrm{AB}}=14.1 \mathrm{~Hz}\right.$, in addition split by $J_{5-\mathrm{CH}^{\mathrm{A}}, 5}=4.4 \mathrm{~Hz}, J_{5-\mathrm{CH}^{\mathrm{B}}, 5}=6.0 \mathrm{~Hz}, 2 \mathrm{H}$, 5- $\mathrm{CH}_{2}$ ), 4.06 (br. s, 1H, 5-H), 5.59 (d, $\left.J_{7^{\prime}, 8^{\prime}}=9.8 \mathrm{~Hz}, 1 \mathrm{H}, 7^{\prime}-\mathrm{H}\right), 6.45$ (dd, $J_{4^{\prime}, 3^{\prime}}=11.3 \mathrm{~Hz}$, $\left.J_{4^{\prime}, 5^{\prime}}=15.2 \mathrm{~Hz}, 1 \mathrm{H}, 4^{\prime}-\mathrm{H}\right), 6.63-6.67$ (m, 2H, 2"-H, 6"-H), 6.77 (d, $\left.J_{5^{\prime}, 4^{\prime}}=15.1 \mathrm{~Hz}, 1 \mathrm{H}, 5^{\prime}-\mathrm{H}\right)$, 6.96-6.99 (m, 2H, 3"-H, 5"-H), $7.10\left(\mathrm{~d}, J_{2^{\prime}, 3^{\prime}}=15.2 \mathrm{~Hz}, 1 \mathrm{H}, 2^{\prime}-\mathrm{H}\right), 7.54$ (dd, $J_{3^{\prime}, 4^{\prime}}=11.2 \mathrm{~Hz}$, $\left.J_{3^{\prime}, 2^{\prime}}=15.2 \mathrm{~Hz}, 1 \mathrm{H}, 3^{\prime}-\mathrm{H}\right)$.

${ }^{13}$ C NMR (125.75 MHz, MeOD/MeOH, DsBrSe25-201104, 26.9.2019): $\delta=11.7$ (C-12'), 12.6 (6'- $\left.\mathrm{CH}_{3}\right), 19.5\left(10^{\prime}-\mathrm{CH}_{3}\right), 21.5\left(8^{\prime}-\mathrm{CH}_{3}\right), 31.2\left(\mathrm{C}-11\right.$ '), $32.1\left(\mathrm{C}-8^{\prime}\right), 33.7\left(\mathrm{C}-10\right.$ '), $37.6\left(5-\mathrm{CH}_{2}\right)$, 45.8 (C-9'), 64.2 (C-5), 116.1 (C-2", C-6"), 120.7 (C-2'), 126.3 (C-4'), 127.7 (C-4"), 131.8 (C3", C-5"), 134.3 (C-6'), 147.3 (C-3'), 148.4 (C-7'), 150.3 (C-5'), 157.3 (C-1"), 175.3 (C-2).* *C-3, C-4 and C-1' were not found.

edHSQC [,,short-range H,C-COSY“, 500.10 MHz/125.75 MHz, MeOD; $\delta\left({ }^{1} \mathrm{H}\right) \leftrightarrow \delta\left({ }^{13} \mathrm{C}\right)$ : 0.84-0.88 (m, 10'- $\left.\mathrm{CH}_{3}\right) \leftrightarrow 19.5\left(10^{\prime}-\mathrm{CH}_{3}\right), 0.80-0.87\left(\mathrm{~m}, 12^{\prime}-\mathrm{H}_{3}\right) \leftrightarrow 11.7\left(\mathrm{C}-12^{\prime}\right), 0.98\left(\mathrm{~d}, 8^{\prime}-\right.$ 
$\left.\mathrm{CH}_{3}\right) \leftrightarrow 21.5\left(8^{\prime}-\mathrm{CH}_{3}\right), 1.10-1.19\left(\mathrm{~m}, 9^{\prime}-\mathrm{H}^{\mathrm{A}}\right) \leftrightarrow 45.8\left(\mathrm{C}-9^{\prime}\right), 1.10-1.19\left(\mathrm{~m}, 11^{\prime}-\mathrm{H}^{\mathrm{A}}\right) \leftrightarrow 31.2(\mathrm{C}-$ $\left.11^{\prime}\right), 1.22-1.38\left(\mathrm{~m}, 10^{\prime}-\mathrm{H}\right) \leftrightarrow 33.7\left(\mathrm{C}-10^{\prime}\right), 1.22-1.38\left(\mathrm{~m}, 9^{\prime}-\mathrm{H}^{\mathrm{B}}\right) \leftrightarrow 45.8\left(\mathrm{C}-9^{\prime}\right), 1.22-1.38(\mathrm{~m}$, $\left.11^{\prime}-\mathrm{H}^{\mathrm{B}}\right) \leftrightarrow 31.2\left(\mathrm{C}-11^{\prime}\right), 1.85\left(\mathrm{~d}, 6^{\prime}-\mathrm{CH}_{3}\right) \leftrightarrow 12.6\left(6^{\prime}-\mathrm{CH}_{3}\right), 2.70\left(\mathrm{~m}_{\mathrm{c}}, 8^{\prime}-\mathrm{H}\right) \leftrightarrow 32.1\left(\mathrm{C}-8^{\prime}\right), \mathrm{AB}$ signal $\left(\delta_{\mathrm{A}}=2.87, \delta_{\mathrm{B}}=3.98,5-\mathrm{CH}_{2}\right) \leftrightarrow 37.6\left(5-\mathrm{CH}_{2}\right), 4.06$ (br. s, 5-H) $64.2(\mathrm{C}-5), 5.59(\mathrm{~d}$, 7'-H), ↔ 148.4 (C-7'), 6.45 (dd, 4'-H) ↔ 126.3 (C-4'), 6.63-6.67 (m, 2"-H, 6"-H) 116.1 (C2", C-6"), 6.77 (d, 5'-H) 150.3 (C-5'), 6.96-6.99 (m, 3"-H, 5"-H) ↔ 131.8 (C-3", C-5"), 7.10 $\left(\mathrm{d}, 2^{\prime}-\mathrm{H}\right) \leftrightarrow 120.7\left(\mathrm{C}-2^{\prime}\right), 7.54\left(\mathrm{dd}, 3^{\prime}-\mathrm{H}\right) \leftrightarrow 147.3\left(\mathrm{C}-3^{\prime}\right)$.

Specific rotation $(S, R, R)-\mathbf{1}[a]_{D}^{20}=-317.0^{\circ}(\mathrm{c}=0.17$ in $\mathrm{MeOH})$.

Specific rotation $(R, S, S)-\mathbf{1}[a]_{D}^{20}=319.0^{\circ}(\mathrm{c}=0.17$ in $\mathrm{MeOH})$.

HRMS (ESI) m/z: [M-H] ${ }^{+}$Calcd for $\mathrm{C}_{26} \mathrm{H}_{32} \mathrm{O}_{4} \mathrm{~N}$ 422.2337; Found 422.2332

IR (KBr): $\tilde{v}=3290,3070,3030,2960,2925,2870,2855,1655,1625,1595,1550,1510,1430$, $1370,1310,1230,1205,1175,1105,1030,1000,955,895,870,815,610 \mathrm{~cm}^{-1}$. 
Christian Drescher, Morris Keller, Olivier Potterat, Matthias Hamburger and Reinhard Brückner, Structure-Elucidating Total Synthesis of the (Polyenoyl)tetramic Acid Militarinone C

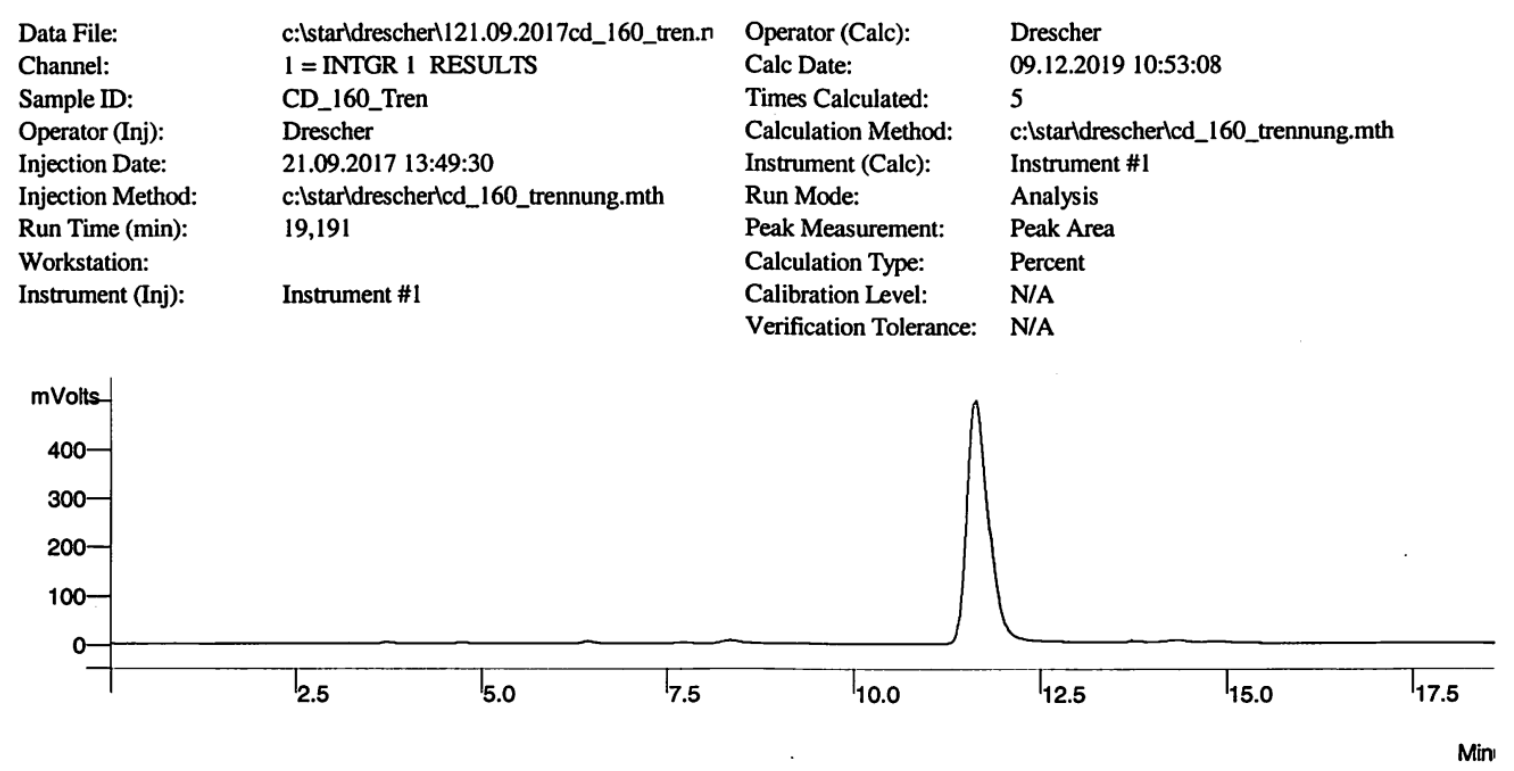

\begin{tabular}{|c|c|c|c|c|c|c|c|c|c|c|}
\hline $\begin{array}{c}\text { Peak } \\
\text { No }\end{array}$ & Peak Name & Result () & $\begin{array}{c}\text { Ret. } \\
\text { Time } \\
\text { (min) }\end{array}$ & $\begin{array}{l}\text { Time } \\
\text { Offiset } \\
\text { (min) }\end{array}$ & $\begin{array}{c}\text { Area } \\
\text { (counts) }\end{array}$ & $\begin{array}{c}\text { Rel } \\
\text { Ret } \\
\text { Time }\end{array}$ & $\begin{array}{l}\text { Sep. } \\
\text { Code }\end{array}$ & $\begin{array}{c}\text { Width } \\
1 / 2 \\
\text { (sec) }\end{array}$ & $\begin{array}{l}\text { Status } \\
\text { Codes }\end{array}$ & Group \\
\hline 1 & & 0,2814 & 3,697 & 0,000 & 29059 & 0,00 & BB & 9,6 & & 0 \\
\hline 2 & & 0,1139 & 4,725 & 0,000 & 11759 & 0,00 & BB & 12,0 & & 0 \\
\hline 3 & & 0,4873 & 6,420 & 0,000 & 50319 & 0,00 & BB & 10,1 & & 0 \\
\hline 4 & & 0,1952 & 7,696 & 0,000 & 20158 & 0,00 & BB & 10,6 & . & 0 \\
\hline 5 & & 0,8458 & 8,333 & 0,000 & 87351 & 0,00 & BB & 14,3 & & 0 \\
\hline 6 & & 95,9660 & 11,640 & 0,000 & 9910549 & 0,00 & BB & 19,4 & $\mathrm{U}$ & 0 \\
\hline 7 & & 2,1105 & 14,350 & 0,000 & 217949 & 0,00 & BB & 17,1 & & 0 \\
\hline & & 100,0001 & & 0,000 & 10327144 & & & & & \\
\hline
\end{tabular}

Status Codes:

U - User defined peak endpoint(s) 


\subsection{Isolation and Oxidative Degradation of Natural Militarinone C}

(5S)-5-(1''-Hydroxybenzyl)-3-(1'Z,2' $\left.E, 4^{\prime} E, 6^{\prime} E, 8^{\prime} R, 10 ' R\right)-1^{\prime}-h y d r o x y-6 ', 8^{\prime}, 10^{\prime}-$ trimethyldodeca-2',4',6'-trien-1'-ylidene)pyrrolidine-2,4-dion [Z-enol-(S,R,R)-1] in an 80:20 mixture with

(5S)-5-(1'-Hydroxybenzyl)-3-(1'E,2' $\left.E, 4^{\prime} E, 6^{\prime} E, 8^{\prime} R, 10 ' R\right)-1^{\prime}-h y d r o x y-6 ', 8 ', 10 '-$ trimethyldodeca-2',4',6'-trien-1'-ylidene)pyrrolidine-2,4-dion [E-enol-(S,R,R)-1] and

(5S)-5-[1'"-Hydroxy(1'-hydroxybenzyl)]-3-(1'Z,2' $\left.E, 4^{\prime} E, 6^{\prime} E, 8^{\prime} R, 10 ' R\right)-1^{\prime}-h y d r o x y-$ $6^{\prime}, 8^{\prime}, 10$ '-trimethyldodeca-2',4',6'-trien-1'-ylidene)pyrrolidine-2,4-dion [Z-enol-( $\left.S, R, R\right)$-2] in an 79:21 mixture with

$(5 S)-5-\left[1^{\prime \prime \prime}-H y d r o x y\left(1^{\prime}\right.\right.$ '-hydroxybenzyl)]-3-(1'E,2' $\left.E, 4^{\prime} E, 6^{\prime} E, 8^{\prime} R, 10 ' R\right)-1 '-h y d r o x y-$ $6^{\prime}, 8^{\prime}, 10 '$-trimethyldodeca-2',4',6'-trien-1'-ylidene)pyrrolidine-2,4-dion [E-enol-( $\left.S, R, R\right)$-2] and

4-Hydroxy-5-(1"'-hydroxyphenyl)-3-[(2E,4E,6E,8R,10R)-6,8,10-trimethyldodeca-2,4,6trienoyl]pyridin-2(1H)-one $[(R, R)-3]$

and

5-[(1"s,4"S)-1",4"'-Dihydroxycyclohexyl]-4-hydroxy-3-[(2E,4E,6E,8R,10R)-6,8,10trimethyldodeca-2,4,6-trienoyl]pyridin-2(1H)-one $[(R, R)-4]$

and

5-[(1"s,4"S)-1",4"'-Dihydroxycyclohexyl]-1,4-dihydroxy-3-[(2E,4E,6E,8R,10R)-6,8,10trimethyldodeca-2,4,6-trienoyl]pyridin-2(1H)-one $[(R, R)-5]$ 

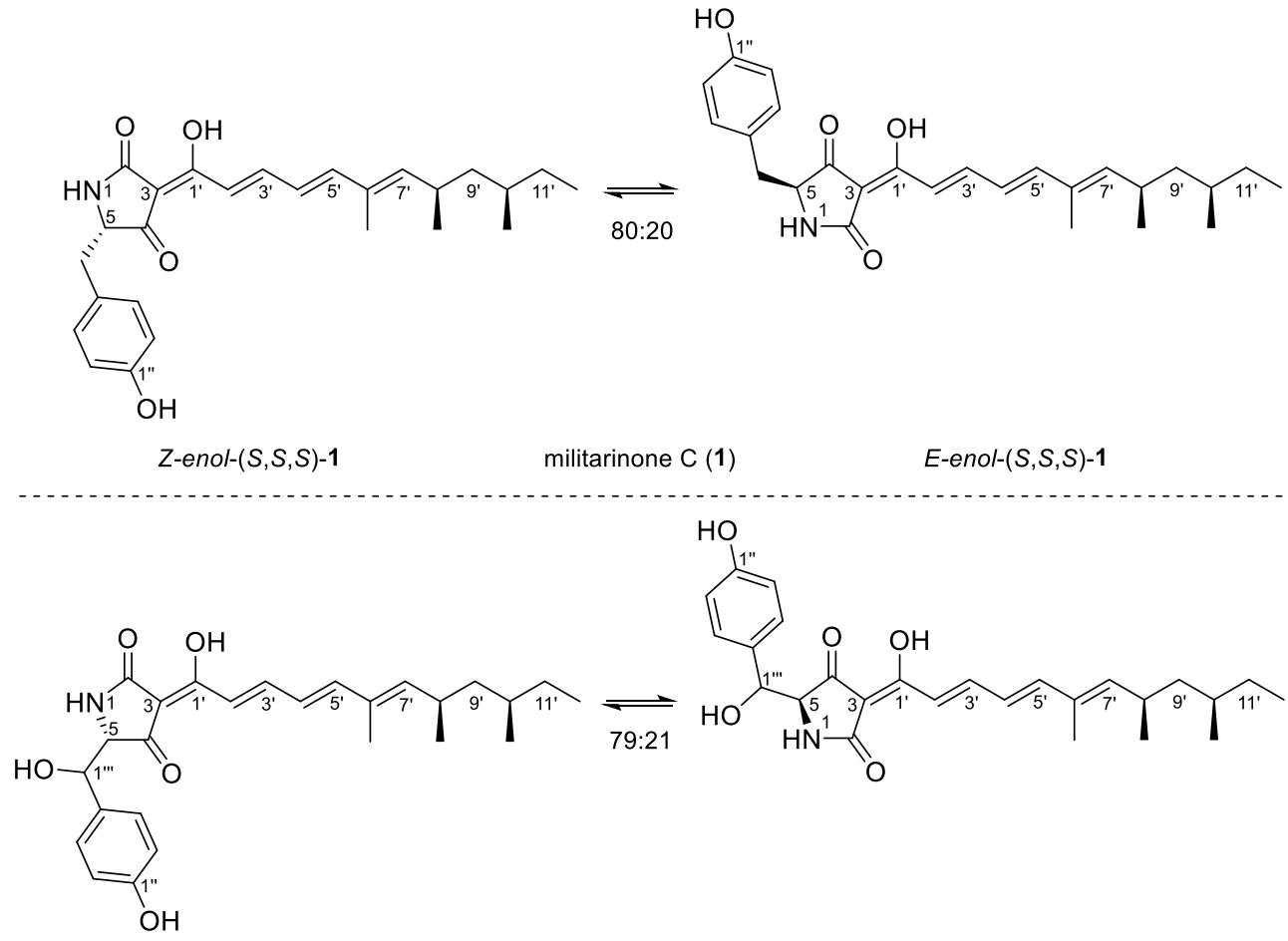

Z-enol-(S,R,R)-2 militarinone B (2)

E-enol-(S,R,R)-2
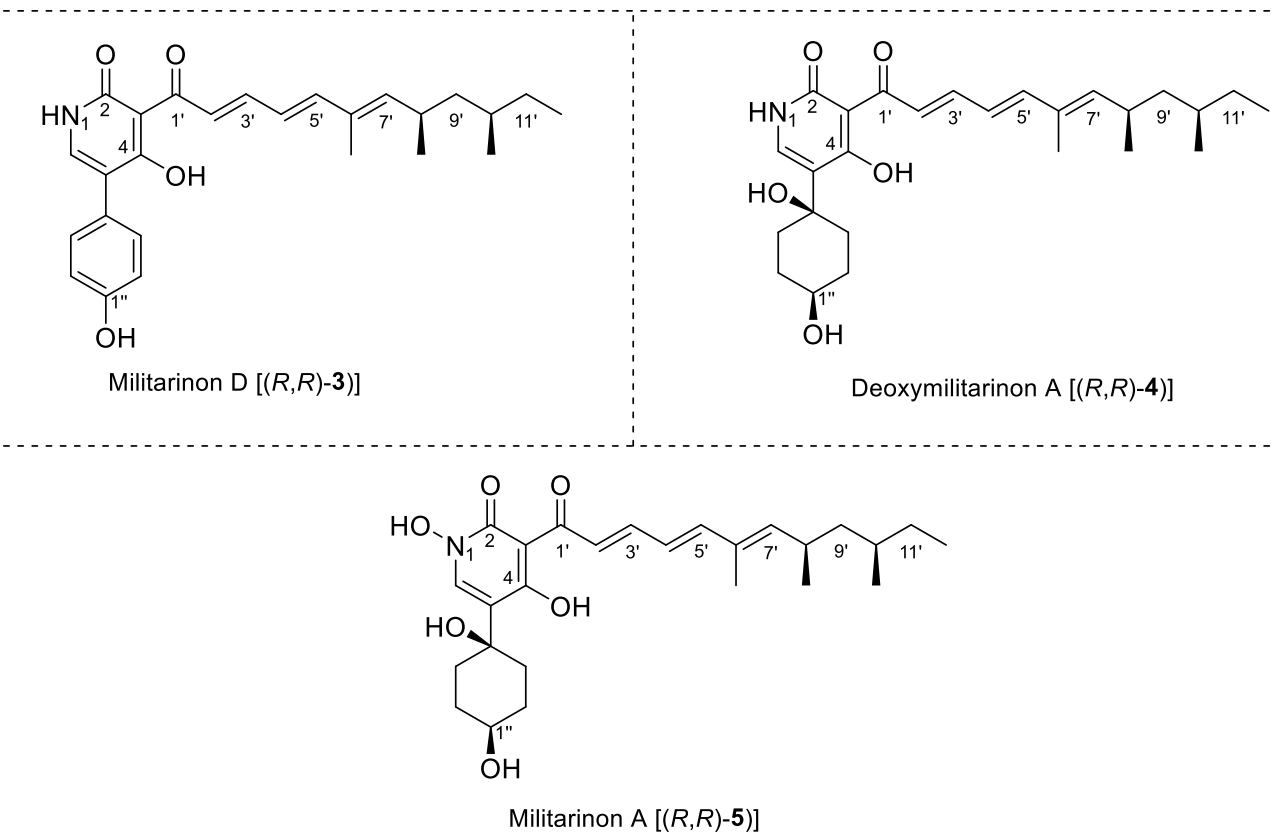

The fungus Paecilomyces militaris RCEF 0095 was cultivated on agar slants [sucrose (40 g/L), peptone $(10 \mathrm{~g} / \mathrm{L})$, yeast extract $(10 \mathrm{~g} / \mathrm{L})$ and agar $(20 \mathrm{~g} / \mathrm{L})]$ for $10 \mathrm{~d}$ at $25^{\circ} \mathrm{C}$. For fermentation, mycelia pieces were transferred into Erlenmeyer flasks $(500 \mathrm{~mL})$ with liquid medium [56 $\times 150 \mathrm{~mL}$; glucose $(20 \mathrm{~g} / \mathrm{L})$, neopeptone $(20 \mathrm{~g} / \mathrm{L})$, glycine $(5 \mathrm{~g} / \mathrm{L}), \mathrm{K}_{2} \mathrm{HPO}_{4}(2 \mathrm{~g} / \mathrm{L})$ and $\left.\mathrm{MgSO}_{4} \times 7 \mathrm{H}_{2} \mathrm{O}(1 \mathrm{~g} / \mathrm{L})\right]$. Still cultures were kept at $27{ }^{\circ} \mathrm{C}$ for $32 \mathrm{~d}$. Mycelium and broth were separated by filtration and the mycelium was freeze-dried to afford a solid residue $(44.2 \mathrm{~g})$. It was extracted with $\mathrm{MeOH}(3 \times 1 \mathrm{~L})$ for $24 \mathrm{~h}$ at $25{ }^{\circ} \mathrm{C}$ under light protection. The combined extracts were filtered und the solvent was removed under reduced pressure. The residue was 
freeze-dried to give a red solid (25.9 g). It was diluted with $\mathrm{H}_{2} \mathrm{O}(500 \mathrm{~mL})$. After ultrasonication and centrifugation at $10{ }^{\circ} \mathrm{C}$ and $800 \mathrm{U} / \mathrm{min}$, the aq. supernatant was decanted. The insoluble gum was freeze-dried $(5.20 \mathrm{~g})$. The residue was extracted with $\mathrm{MeOH}(15 \mathrm{~mL})$ and centrifuged as described. The $\mathrm{MeOH}$ was removed under reduced pressure and the residue (3.52 $\mathrm{g}$ ) was submitted to gel permeation chromatography on a Sephadex LH-20 columm $(20-100 \mu \mathrm{m}$, $\mathrm{MeOH}, 100 \mathrm{~cm}, 5.5 \mathrm{~cm}, 1.5 \mathrm{~mL} / \mathrm{min}, 20 \mathrm{~mL}$ ). On basis of TLC-pattern three fractions (A $\rightarrow$ C) were collected [fraction A (88-104, $750 \mathrm{mg})$; fraction B (109-129, $212 \mathrm{mg})$; fraction C (152164, $8.0 \mathrm{mg})$ ]. The residue of fraction A was purified by preparative HPLC [SunFire ${ }^{\mathrm{TM}}$ Prep C18 OBDTM $(5 \mu \mathrm{m}, 30 \times 150 \mathrm{~mm}$ i.d., Waters), equipped with a C18 Prep Guard Cartridge $(10 \times 30$ mm i.d. $) ; \mathrm{MeCN}: \mathrm{H}_{2} \mathrm{O}: \mathrm{HCO}_{2} \mathrm{H}=70: 30: 0.1 \rightarrow 100: 0: 0.1,0 \mathrm{~min} \rightarrow 30 \mathrm{~min}, 20 \mathrm{~mL} / \mathrm{min}$, injections of $72 \mathrm{mg}$ of crude product per run, $25^{\circ} \mathrm{C}, \lambda \mathrm{UV} / \mathrm{vis}$-detector $=400 \mathrm{~nm}$; $\operatorname{tretention,~}(R, R)-4=14.9$ min; $\left.t_{\text {retention, }(R, R)-5}=16.5 \mathrm{~min}\right]$. Deoxymilitarinone A $[(R, R)-4](11.5 \mathrm{mg}, 26.1 \mu \mathrm{mol})$ was obtained as an orange powder. Militarinone A $[(R, R)-5](177.0 \mathrm{mg}, 386.0 \mu \mathrm{mol})$ was also obtained as an orange powder. The residue of fraction B was although purified by preparative HPLC [SunFire ${ }^{\mathrm{TM}}$ Prep C18 OBDTM $(5 \mu \mathrm{m}, 30 \times 150 \mathrm{~mm}$ i.d., Waters $)$, equipped with a C18 Prep Guard Cartridge $\left(10 \times 30 \mathrm{~mm}\right.$ i.d.); $\mathrm{MeCN}: \mathrm{H}_{2} \mathrm{O}: \mathrm{HCO}_{2} \mathrm{H}=75: 25: 0.1 \rightarrow$ 100:0:0.1, 0 min $\rightarrow 30 \mathrm{~min}, 20 \mathrm{~mL} / \mathrm{min}$, injections of $12 \mathrm{mg}$ of crude product per run, $25^{\circ} \mathrm{C}, \lambda_{\mathrm{UV} / \text { vis-detector }}=400$

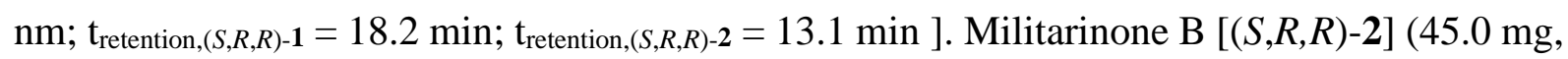
$102 \mu \mathrm{mol})$ and militarinone $\mathrm{C}[(S, R, R)-1](63.2 \mathrm{mg}, 150 \mu \mathrm{mol})$ were both obtained as a yellow powder. The residue of fractions $C$ was purified by semipreparative HPLC [SunFire ${ }^{\mathrm{TM}}$ Prep C18 OBDTM (5 $\mu \mathrm{m}, 10 \times 150 \mathrm{~mm}$ i.d., Waters), equipped with a C18 Prep Guard Cartridge (10 x $10 \mathrm{~mm}$ i.d.); $\mathrm{MeCN}: \mathrm{H}_{2} \mathrm{O}: \mathrm{HCO}_{2} \mathrm{H}=72: 28: 0.1,4 \mathrm{~mL} / \mathrm{min}$, injections of $2 \mathrm{mg}$ of crude product per run, $25^{\circ} \mathrm{C}, \lambda_{\mathrm{UV} / \mathrm{vis} \text {-detector }}=400 \mathrm{~nm}$; $\left.\mathrm{t}_{\text {retention, }(R, R)-3}=19.9 \mathrm{~min}\right]$. Militarinone $\mathrm{D}[(R, R)$ 3] $(2.4 \mathrm{mg}, 6.0 \mu \mathrm{mol})$ was obtained as an orange powder.

Militarinone $\mathrm{C}[(S, R, R)-\mathbf{1}]^{17}$ :

For characterization data: p. 60

Specific rotation $(S, R, R)-\mathbf{1}$ (natural) $[a]_{D}^{20}=-307^{\circ}(\mathrm{c}=0.17$ in $\mathrm{MeOH})$

Militarinone $\mathrm{B}[(S, R, R)-2]^{17}$ :

${ }^{1}$ H NMR (500.10 MHz, MeOD/MeOH, DsBrSe13-500200, 16.9.2019): 0.84-0.87 (m, 3H, 10'$\left.\mathrm{CH}_{3}\right), 0.80-0.87\left(\mathrm{~m}, 3 \mathrm{H}, 12^{\prime}-\mathrm{H}_{3}\right), 0.98\left(\mathrm{~d}, J_{8^{\prime}-\mathrm{CH}_{3}, 8^{\prime}}=6.6 \mathrm{~Hz}, 3 \mathrm{H}, 8^{\prime}-\mathrm{CH}_{3}\right), 1.10-1.19\left(\mathrm{~m}, 1 \mathrm{H}, 9^{\prime}-\right.$ $\left.\mathrm{H}^{\mathrm{A}}\right), 1.10-1.19\left(\mathrm{~m}, 1 \mathrm{H}, 11^{\prime}-\mathrm{H}^{\mathrm{A}}\right), 1.22-1.38\left(\mathrm{~m}, 1 \mathrm{H}, 10^{\prime}-\mathrm{H}\right), 1.22-1.38\left(\mathrm{~m}, 1 \mathrm{H}, 9^{\prime}-\mathrm{H}^{\mathrm{B}}\right), 1.22-1.38$ 
$\left(\mathrm{m}, 1 \mathrm{H}, 11^{\prime}-\mathrm{H}^{\mathrm{B}}\right), 1.86\left(\mathrm{~d}, J_{6}-\mathrm{CH}_{3}, 7^{\prime}=1.1 \mathrm{~Hz}, 3 \mathrm{H}, 6^{\prime}-\mathrm{CH}_{3}\right), 2.71\left(\mathrm{~m}_{\mathrm{c}}, 1 \mathrm{H}, 8^{\prime}-\mathrm{H}\right), 4.23($ br. $\mathrm{s}, 1 \mathrm{H}, 5-$ H), $4.99\left(\mathrm{~d}, J_{1^{\prime \prime}, 5}=3.8 \mathrm{~Hz}, 1 \mathrm{H}, 1^{\prime \prime \prime}-\mathrm{H}\right), 5.59\left(\mathrm{~d}, J_{7^{\prime}, 8^{\prime}}=9.8 \mathrm{~Hz}, 1 \mathrm{H}, 7^{\prime}-\mathrm{H}\right), 6.44\left(\mathrm{dd}, J_{4^{\prime}, 3^{\prime}}=11.2 \mathrm{~Hz}\right.$, $\left.J_{4^{\prime}, 5^{\prime}}=15.1 \mathrm{~Hz}, 1 \mathrm{H}, 4^{\prime}-\mathrm{H}\right), 6.65-6.69$ (m, 2H, 2"-H, 6"-H), 6.76 (d, $\left.J_{5^{\prime}, 4^{\prime}}=15.1 \mathrm{~Hz}, 1 \mathrm{H}, 5^{\prime}-\mathrm{H}\right)$, $7.03\left(\mathrm{~d}, J_{2^{\prime}, 3^{\prime}}=15.2 \mathrm{~Hz}, 1 \mathrm{H}, 2^{\prime}-\mathrm{H}\right), 7.10-7.14\left(\mathrm{~m}, 2 \mathrm{H}, 3 "-\mathrm{H}, 5^{\prime \prime}-\mathrm{H}\right), 7.50\left(\mathrm{dd}, J_{3^{\prime}, 4^{\prime}}=11.2 \mathrm{~Hz}\right.$, $\left.J_{3^{\prime}, 2^{\prime}}=15.1 \mathrm{~Hz}, 1 \mathrm{H}, 3^{\prime}-\mathrm{H}\right)$.

${ }^{13} \mathrm{C}$ NMR (125.75 MHz, MeOD/MeOH, DsBrSe13-500207, 16.9.2019): $\delta$ = 11.6 (C-12'), 12.6 (6'- $\left.\mathrm{CH}_{3}\right), 19.5\left(10^{\prime}-\mathrm{CH}_{3}\right), 21.5\left(8^{\prime}-\mathrm{CH}_{3}\right), 31.2\left(\mathrm{C}-11^{\prime}\right), 32.1\left(\mathrm{C}-8^{\prime}\right), 33.7(\mathrm{C}-10 '), 45.8$ (C-9'), 68.7 (C-5), 74.8 (C-1"'), 115.6 (C-2", C-6"), 120.7 (C-2'), 126.3 (C-4'), 129.6 (C-3", C-5"), 130.6 (C4"), 134.3 (C-6'), 147.2 (C-3'), 148.3 (C-7'), 150.3 (C-5'), 158.2 (C-1"), 175.3 (C-2).*

${ }^{*} \mathrm{C}-3, \mathrm{C}-4$ and C-1' were not found.

edHSQC [,,short-range H,C-COSY“, 500.10 MHz/125.75 MHz, MeOD; $\delta\left({ }^{1} \mathrm{H}\right) \leftrightarrow \delta\left({ }^{13} \mathrm{C}\right)$ : 0.84-0.87 (m, 12'- $\left.\mathrm{CH}_{3}\right) \leftrightarrow 11.6\left(\mathrm{C}-12^{\prime}\right), 0.80-0.87\left(\mathrm{~m}, 10^{\prime}-\mathrm{H}_{3}\right) \leftrightarrow 19.5\left(10^{\prime}-\mathrm{CH}_{3}\right), 0.98\left(\mathrm{~d}, 8^{\prime}-\right.$ $\left.\mathrm{CH}_{3}\right) \leftrightarrow 21.5\left(8^{\prime}-\mathrm{CH}_{3}\right), 1.10-1.19\left(\mathrm{~m}, 9^{\prime}-\mathrm{H}^{\mathrm{A}}\right) \leftrightarrow 45.8\left(\mathrm{C}-9^{\prime}\right), 1.10-1.19\left(\mathrm{~m}, 11^{\prime}-\mathrm{H}^{\mathrm{A}}\right) \leftrightarrow 31.2(\mathrm{C}-$ $\left.11^{\prime}\right), 1.22-1.38\left(\mathrm{~m}, 10^{\prime}-\mathrm{H}\right) \leftrightarrow 33.7\left(\mathrm{C}-10^{\prime}\right), 1.22-1.38\left(\mathrm{~m}, 9^{\prime}-\mathrm{H}^{\mathrm{B}}\right) \leftrightarrow 45.8(\mathrm{C}-9$ ') $1.22-1.38(\mathrm{~m}$, $\left.11^{\prime}-\mathrm{H}^{\mathrm{B}}\right) \leftrightarrow 31.2\left(\mathrm{C}-11^{\prime}\right), 1.86\left(\mathrm{~d}, 6^{\prime}-\mathrm{CH}_{3}\right) \leftrightarrow 12.6\left(6^{\prime}-\mathrm{CH}_{3}\right), 2.71\left(\mathrm{~m}_{\mathrm{c}}, 8^{\prime}-\mathrm{H}\right) \leftrightarrow 32.1\left(\mathrm{C}-8^{\prime}\right), 4.23$ (br. s, 5-H) ↔ 68.7 (C-5), 4.99 (d, 1"'-H) ↔ 74.8 (C-1'"), 5.59 (d, 7'-H) ↔ 148.3 (C-7'), 6.44 $\left(\mathrm{dd}, 4{ }^{\prime}-\mathrm{H}\right) \leftrightarrow 126.7$ (C-4'), 6.65-6.69 (m, 2"-H, 6"-H) ↔ 115.6 (C-2", C-6"), 6.76 (d, 5'-H) 150.3 (C-5'), 7.03 (d, 2'-H) ↔ 120.7 (C-2'), 7.10-7.14 (m, 3"-H, 5"-H) ↔ 129.6 (C-3", C-5"), $7.50\left(\mathrm{dd}, 3^{\prime}-\mathrm{H}\right) \leftrightarrow 147.2\left(\mathrm{C}-3^{\prime}\right)$.

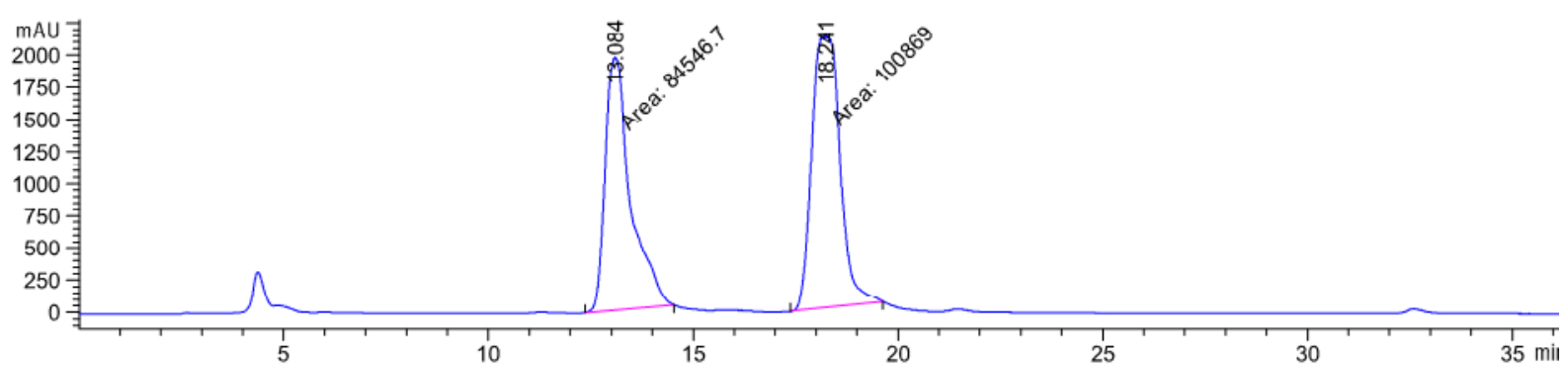

Isolation of militarinone $B(2), t_{R}=13.1 \mathrm{~min}$ and militarinone $C(1), t_{R}=18.2 \mathrm{~min}(U \mathrm{~V}$-detection: $400 \mathrm{~nm})$.

Specific rotation $(S, R, R)-2[a]_{D}^{20}=-282^{\circ}(\mathrm{c}=0.19$ in $\mathrm{MeOH})$

HRMS (ESI) m/z: [M-H] ${ }^{+}$Calcd for $\mathrm{C}_{26} \mathrm{H}_{32} \mathrm{O}_{5} \mathrm{~N} 438.2286$; Found 438.2287 
Christian Drescher, Morris Keller, Olivier Potterat, Matthias Hamburger and Reinhard Brückner, Structure-Elucidating Total Synthesis of the (Polyenoyl)tetramic Acid Militarinone C

Militarinone $\mathrm{D}[(R, R)-3]^{17}$ :

${ }^{1} \mathbf{H}$ NMR (500.10 MHz, MeOD/MeOH, DsBrSe20-500600, 23.9.2019): 0.86 (d, $J_{10}$ '$\left.\mathrm{CH}_{3}, 10^{\prime}=6.3 \mathrm{~Hz}, 3 \mathrm{H}, 10^{\prime}-\mathrm{CH}_{3}\right), 0.87\left(\mathrm{t}, J_{12^{\prime}, 11^{\prime}}=7.3 \mathrm{~Hz}, 3 \mathrm{H}, 12^{\prime}-\mathrm{H}_{3}\right), 0.98\left(\mathrm{~d}, J_{8^{\prime}-\mathrm{CH}_{3}, 8^{\prime}}=6.6 \mathrm{~Hz}\right.$, $\left.3 \mathrm{H}, 8^{\prime}-\mathrm{CH}_{3}\right), 1.11-1.19\left(\mathrm{~m}, 1 \mathrm{H}, 9^{\prime}-\mathrm{H}^{\mathrm{A}}\right), 1.11-1.19\left(\mathrm{~m}, 1 \mathrm{H}, 11^{\prime}-\mathrm{H}^{\mathrm{A}}\right), 1.23-1.38(\mathrm{~m}, 1 \mathrm{H}, 10 '-\mathrm{H})$, 1.23-1.38 (m, $\left.1 \mathrm{H}, 9^{\prime}-\mathrm{H}^{\mathrm{B}}\right), 1.23-1.38\left(\mathrm{~m}, 1 \mathrm{H}, 11^{\prime}-\mathrm{H}^{\mathrm{B}}\right), 1.86\left(\mathrm{~d}, J_{6^{\prime}}-\mathrm{CH}_{3}, 7^{\prime}=0.8 \mathrm{~Hz}, 3 \mathrm{H}, 6^{\prime}-\mathrm{CH}_{3}\right)$, $2.70\left(\mathrm{~m}_{\mathrm{c}}, 1 \mathrm{H}, 8^{\prime}-\mathrm{H}\right), 4.56$ (br. s, 2H, NH, 1"-OH), 5.56 (d, J $\left.J^{\prime}, 8^{\prime}=9.8 \mathrm{~Hz}, 1 \mathrm{H}, 7^{\prime}-\mathrm{H}\right), 6.48$ (dd, $\left.J_{4^{\prime}, 3^{\prime}}=11.3 \mathrm{~Hz}, J_{4^{\prime}, 5^{\prime}}=15.3 \mathrm{~Hz}, 1 \mathrm{H}, 4^{\prime}-\mathrm{H}\right), 6.77\left(\mathrm{~d}, J_{5^{\prime}, 4^{\prime}}=15.3 \mathrm{~Hz}, 1 \mathrm{H}, 5^{\prime}-\mathrm{H}\right), 6.79-6.82(\mathrm{~m}, 2 \mathrm{H}$, 2"-H, 6"-H), 7.26-7.39 (m, 2H, 3"-H, 5"-H), 7.46 (br. s, 1H, 6-H), 7.63 (dd, J' $3^{\prime}, 4^{\prime}=11.4$ Hz, $\left.J_{3^{\prime}, 2^{\prime}}=14.9 \mathrm{~Hz}, 1 \mathrm{H}, 3^{\prime}-\mathrm{H}\right), 7.97\left(\mathrm{~d}, J_{2^{\prime}, 3^{\prime}}=14.9 \mathrm{~Hz}, 1 \mathrm{H}, 2^{\prime}-\mathrm{H}\right)$.

${ }^{13} \mathrm{C}$ NMR (125.75 MHz, MeOD/MeOH, DsBrSe20-500603, 23.9.2019): $\delta=11.7$ (C-12'), 12.6 (6'- $\left.\mathrm{CH}_{3}\right), 19.5\left(10^{\prime}-\mathrm{CH}_{3}\right), 21.6\left(8^{\prime}-\mathrm{CH}_{3}\right), 31.2\left(\mathrm{C}-11\right.$ '), $32.0\left(\mathrm{C}-8^{\prime}\right), 33.7$ (C-10'), 45.9 (C-9'), 116.1 (C-2", C-6"), 126.5 (C-4'), 128.6 (C-2'), 131.4 (C-3", C-5"), 134.2 (C-6'), 140.3 (C-6), 147.3 (C-7', C-3'), 149.5 (C-5'), 158.4 (C-2).

*C-3, C-4, C-5, C-1', C-1" and C-4" were not found.

edHSQC [,short-range H,C-COSY“, 500.10 MHz/125.75 MHz, MeOD; $\delta\left({ }^{1} \mathrm{H}\right) \leftrightarrow \delta\left({ }^{13} \mathrm{C}\right)$ : $0.86\left(\mathrm{~d}, 10^{\prime}-\mathrm{CH}_{3}\right) \leftrightarrow 19.5\left(10^{\prime}-\mathrm{CH}_{3}\right), 0.87\left(\mathrm{t}, 12^{\prime}-\mathrm{H}_{3}\right) \leftrightarrow 11.7\left(\mathrm{C}-12^{\prime}\right), 0.98\left(\mathrm{~d}, 8^{\prime}-\mathrm{CH}_{3}\right) \leftrightarrow 21.6$ $\left(8^{\prime}-\mathrm{CH}_{3}\right), 1.11-1.19\left(\mathrm{~m}, 9^{\prime}-\mathrm{H}^{\mathrm{A}}\right) \leftrightarrow 45.9\left(\mathrm{C}-9^{\prime}\right), 1.11-1.19\left(\mathrm{~m}, 11^{\prime}-\mathrm{H}^{\mathrm{A}}\right) \leftrightarrow 31.2\left(\mathrm{C}-11^{\prime}\right), 1.23-1.38$ $\left(\mathrm{m}, 10^{\prime}-\mathrm{H}\right) \leftrightarrow 33.7\left(\mathrm{C}-10^{\prime}\right), 1.23-1.38\left(\mathrm{~m}, 9^{\prime}-\mathrm{H}^{\mathrm{B}}\right) \leftrightarrow 45.9\left(\mathrm{C}-9^{\prime}\right), 1.23-1.38\left(\mathrm{~m}, 11^{\prime}-\mathrm{H}^{\mathrm{B}}\right) \leftrightarrow 31.2$ $(\mathrm{C}-11 '), 1.86\left(\mathrm{~d}, 6^{\prime}-\mathrm{CH}_{3}\right) \leftrightarrow 12.6\left(6^{\prime}-\mathrm{CH}_{3}\right), 2.70\left(\mathrm{~m}_{\mathrm{c}}, 8^{\prime}-\mathrm{H}\right) \leftrightarrow 32.0\left(\mathrm{C}-8^{\prime}\right), 5.56\left(\mathrm{~d}, 7^{\prime}-\mathrm{H}\right) \leftrightarrow 147.3$ $\left(\mathrm{C}-7^{\prime}\right), 6.48\left(\mathrm{dd}, 4^{\prime}-\mathrm{H}\right) \leftrightarrow 126.5\left(\mathrm{C}-4^{\prime}\right), 6.77\left(\mathrm{~d}, 5^{\prime}-\mathrm{H}\right) \leftrightarrow 149.5\left(\mathrm{C}-5^{\prime}\right), 6.79-6.82$ (m, 2"-H, 6"H) 116.1 (C-2", C-6"), 7.26-7.39 (m, 3"-H, 5"-H) 131.4 (C-3", C-5"), 7.46 (br. s, 1H, 6$\mathrm{H}) \leftrightarrow 140.3(\mathrm{C}-6), 7.63\left(\mathrm{dd}, 3^{\prime}-\mathrm{H}\right) \leftrightarrow 147.3\left(\mathrm{C}-3{ }^{\prime}\right), 7.97\left(\mathrm{~d}, 2^{\prime}-\mathrm{H}\right) \leftrightarrow 128.6\left(\mathrm{C}-2^{\prime}\right)$.

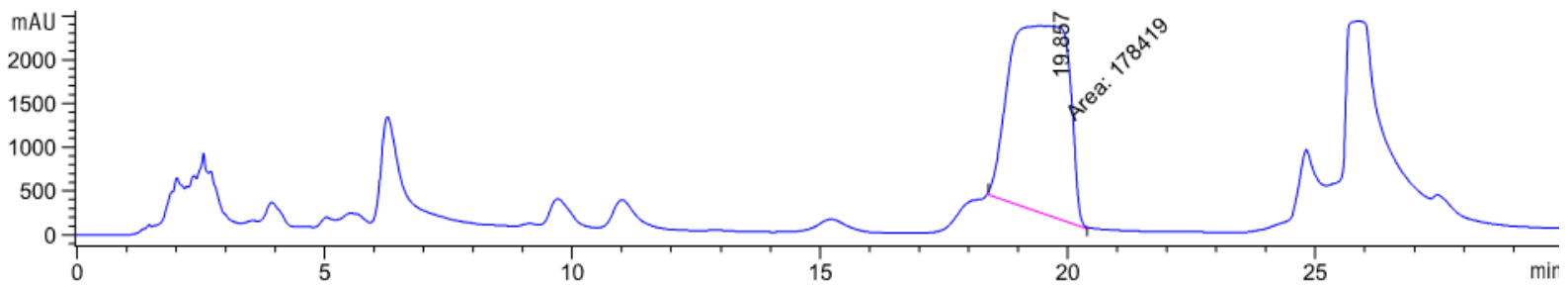
Isolation of militarinone $\mathrm{D}(3), t_{R}=19.9 \min (U V$-detection: $400 \mathrm{~nm})$. 
Christian Drescher, Morris Keller, Olivier Potterat, Matthias Hamburger and Reinhard Brückner, Structure-Elucidating Total Synthesis of the (Polyenoyl)tetramic Acid Militarinone C

Deoxymilitarinone A $[(R, R)-4]^{18}$ :

${ }^{1} \mathbf{H}$ NMR (500.10 MHz, MeOD/MeOH, DsBrSe17-500500, 18.9.2019): 0.85 (d, $J_{10}$ $\left.\mathrm{CH}_{3}, 10^{\prime}=6.3 \mathrm{~Hz}, 3 \mathrm{H}, 10^{\prime}-\mathrm{CH}_{3}\right), 0.86\left(\mathrm{t}, J_{12^{\prime}, 11^{\prime}}=7.3 \mathrm{~Hz}, 3 \mathrm{H}, 12^{\prime}-\mathrm{H}_{3}\right), 0.98\left(\mathrm{~d}, J_{8^{\prime}-\mathrm{CH}_{3}, 8^{\prime}}=6.6 \mathrm{~Hz}\right.$, $\left.3 \mathrm{H}, 8^{\prime}-\mathrm{CH}_{3}\right), 1.10-1.19\left(\mathrm{~m}, 1 \mathrm{H}, 9^{\prime}-\mathrm{H}^{\mathrm{A}}\right), 1.10-1.19\left(\mathrm{~m}, 1 \mathrm{H}, 11^{\prime}-\mathrm{H}^{\mathrm{A}}\right), 1.22-1.38(\mathrm{~m}, 1 \mathrm{H}, 10 \mathrm{\prime}-\mathrm{H})$, $1.22-1.38\left(\mathrm{~m}, 1 \mathrm{H}, 9^{\prime}-\mathrm{H}^{\mathrm{B}}\right), 1.22-1.38\left(\mathrm{~m}, 1 \mathrm{H}, 11^{\prime}-\mathrm{H}^{\mathrm{B}}\right), 1.61\left(\mathrm{~d}, J_{3^{\prime \prime}, 2^{\prime \prime}}=13.1 \mathrm{~Hz}, 1 \mathrm{H}, 3^{\prime \prime}-\mathrm{H}^{\mathrm{A}}\right), 1.61$ $\left(\mathrm{d}, J_{5 ", 6 "}=13.1 \mathrm{~Hz}, 1 \mathrm{H}, 5^{\prime \prime}-\mathrm{H}^{\mathrm{A}}\right), 1.74-1.80\left(\mathrm{~m}, 4 \mathrm{H}, 2^{\prime \prime}-\mathrm{H}_{2}, 6 "-\mathrm{H}_{2}\right), 1.85$ (s, 6'-CH$), 2.39$ (m, 2H, $\left.3^{\prime \prime}-\mathrm{H}^{\mathrm{B}}, 5^{\prime \prime}-\mathrm{H}^{\mathrm{B}}\right), 2.70\left(\mathrm{~m}_{\mathrm{c}}, 1 \mathrm{H}, 8^{\prime}-\mathrm{H}\right), 3.63\left(\mathrm{~m}_{\mathrm{c}}, 1 \mathrm{H}, 1^{\prime \prime}-\mathrm{H}\right), 5.56\left(\mathrm{~d}, J_{7^{\prime}}, 8^{\prime}=9.6 \mathrm{~Hz}, 1 \mathrm{H}, 7^{\prime}-\mathrm{H}\right), 6.46$ $\left(\mathrm{dd}, J_{4^{\prime}, 3^{\prime}}=11.3 \mathrm{~Hz}, J_{4^{\prime}, 5^{\prime}}=15.0 \mathrm{~Hz}, 1 \mathrm{H}, 4^{\prime}-\mathrm{H}\right), 6.77\left(\mathrm{~d}, J_{5^{\prime}, 4^{\prime}}=15.4 \mathrm{~Hz}, 1 \mathrm{H}, 5\right.$ ' $\left.^{\prime} \mathrm{H}\right), 7.58-7.67$ (m, 1H, 6-H), 7.58-7.67 (m, 1H, 3'-H), $7.93\left(\mathrm{~d}, J_{2^{\prime}, 3^{\prime}}=14.9 \mathrm{~Hz}, 1 \mathrm{H}, 2^{\prime}-\mathrm{H}\right)$.

${ }^{13} \mathrm{C}$ NMR (125.75 MHz, MeOD/MeOH, DsBrSe17-500503, 18.9.2019): $\delta=11.7$ (C-12'), 12.6 (6'- $\left.\mathrm{CH}_{3}\right), 19.5\left(10^{\prime}-\mathrm{CH}_{3}\right), 21.6\left(8^{\prime}-\mathrm{CH}_{3}\right), 31.2\left(\mathrm{C}-11\right.$ '), 31.5 (C-2", C-6"), $32.0\left(\mathrm{C}-8^{\prime}\right), 33.7$ (C10'), 34.7 (C-3", C-5"), 45.8 (C-9'), 70.7 (C-1"), 71.7 (C-4"), 107.5 (C-3), 121.3 (C-5), 126.4 (C-4'), 128.4 (C-2'), 134.1 (C-6'), 139.2 (C-6), 147.3 (C-7'), 147.4 (C-3'), 149.6 (C-5'), 164.7 (C-2), 178.7 (C-4), 195.5 (C-1').

edHSQC [,,short-range H,C-COSY“،, 500.10 MHz/125.75 MHz, MeOD; $\delta\left({ }^{1} \mathrm{H}\right) \leftrightarrow \delta\left({ }^{13} \mathrm{C}\right): 0.85$ $\left(\mathrm{d}, 10^{\prime}-\mathrm{CH}_{3}\right) \leftrightarrow 19.5\left(10^{\prime}-\mathrm{CH}_{3}\right), 0.86\left(\mathrm{t}, 12^{\prime}-\mathrm{H}_{3}\right) \leftrightarrow 11.7\left(\mathrm{C}-12^{\prime}\right), 0.98\left(\mathrm{~d}, 8^{\prime}-\mathrm{CH}_{3}\right) \leftrightarrow 21.6\left(8^{\prime}-\right.$ $\left.\mathrm{CH}_{3}\right), 1.11-1.19\left(\mathrm{~m}, 9^{\prime}-\mathrm{H}^{\mathrm{A}}\right) \leftrightarrow 45.9\left(\mathrm{C}-9^{\prime}\right), 1.11-1.19\left(\mathrm{~m}, 11^{\prime}-\mathrm{H}^{\mathrm{A}}\right) \leftrightarrow 31.2\left(\mathrm{C}-111^{\prime}\right), 1.22-1.38(\mathrm{~m}$, $\left.10^{\prime}-\mathrm{H}\right) \leftrightarrow 33.7\left(\mathrm{C}-10^{\prime}\right), 1.22-1.38\left(\mathrm{~m}, 9^{\prime}-\mathrm{H}^{\mathrm{B}}\right) \leftrightarrow 45.9\left(\mathrm{C}-9^{\prime}\right), 1.22-1.38\left(\mathrm{~m}, 11^{\prime}-\mathrm{H}^{\mathrm{B}}\right) \leftrightarrow 31.2(\mathrm{C}-$ $\left.11^{\prime}\right), 1.61\left(\mathrm{~d}, 3 "-\mathrm{H}^{\mathrm{A}}\right) \leftrightarrow 34.7(\mathrm{C}-3 "), 1.61\left(\mathrm{~d}, 5^{\prime \prime}-\mathrm{H}^{\mathrm{A}}\right) \leftrightarrow 34.7(\mathrm{C}-5 "), 1.74-1.80\left(\mathrm{~m}, 2^{\prime \prime}-\mathrm{H}_{2}, 6 "-\mathrm{H}_{2}\right)$ $\leftrightarrow 31.5\left(\mathrm{C}-2^{\prime \prime}, \mathrm{C}-6 "\right), 1.85\left(\mathrm{~d}, 6^{\prime}-\mathrm{CH}_{3}\right) \leftrightarrow 12.6\left(6^{\prime}-\mathrm{CH}_{3}\right), 2.39\left(\mathrm{~m}, 3^{\prime \prime}-\mathrm{H}^{\mathrm{B}}\right) \leftrightarrow 34.7\left(\mathrm{C}-3^{\prime \prime}\right), 2.39$ $\left(\mathrm{m}, 5^{\prime \prime}-\mathrm{H}^{\mathrm{B}}\right) \leftrightarrow 34.7\left(\mathrm{C}-5^{\prime \prime}\right), 2.70\left(\mathrm{~m}_{\mathrm{c}}, 8^{\prime}-\mathrm{H}\right) \leftrightarrow 32.0\left(\mathrm{C}-8^{\prime}\right), 3.63\left(\mathrm{~m}_{\mathrm{c}}, 1^{\prime \prime}-\mathrm{H}\right) \leftrightarrow 70.7\left(\mathrm{C}-1^{\prime \prime}\right), 5.56$ $\left(\mathrm{d}, 7^{\prime}-\mathrm{H}\right) \leftrightarrow 147.4\left(\mathrm{C}-7^{\prime}\right), 6.46\left(\mathrm{dd}, 4^{\prime}-\mathrm{H}\right) \leftrightarrow 126.5\left(\mathrm{C}-4^{\prime}\right), 6.77\left(\mathrm{~d}, 5^{\prime}-\mathrm{H}\right) \leftrightarrow 149.6\left(\mathrm{C}-5^{\prime}\right), 7.58-$ $7.67(\mathrm{~m}, 6-\mathrm{H}) \leftrightarrow 139.2(\mathrm{C}-6), 7.58-7.67\left(\mathrm{~m}, 3^{\prime}-\mathrm{H}\right) \leftrightarrow 147.4\left(\mathrm{C}-3^{\prime}\right), 7.93\left(\mathrm{~d}, 2^{\prime}-\mathrm{H}\right) \leftrightarrow 128.4(\mathrm{C}-$ 2 ').

Militarinone $\mathrm{A}[(R, R)-5]^{17}$ :

${ }^{1} \mathbf{H}$ NMR (500.10 MHz, MeOD/MeOH, DsBrSe16-501100, 17.9.2019): 0.85 (d, $J_{10}$ $\left.\mathrm{CH}_{3}, 10^{\prime}=6.3 \mathrm{~Hz}, 3 \mathrm{H}, 10^{\prime}-\mathrm{CH}_{3}\right), 0.86\left(\mathrm{t}, J_{12^{\prime}, 11^{\prime}}=7.3 \mathrm{~Hz}, 3 \mathrm{H}, 12^{\prime}-\mathrm{H}_{3}\right), 0.98\left(\mathrm{~d}, J_{8^{\prime}-\mathrm{CH}_{3}, 8^{\prime}}=6.6 \mathrm{~Hz}\right.$, $\left.3 \mathrm{H}, 8^{\prime}-\mathrm{CH}_{3}\right), 1.11-1.19\left(\mathrm{~m}, 1 \mathrm{H}, 9^{\prime}-\mathrm{H}^{\mathrm{A}}\right), 1.11-1.19\left(\mathrm{~m}, 1 \mathrm{H}, 11^{\prime}-\mathrm{H}^{\mathrm{A}}\right), 1.22-1.38(\mathrm{~m}, 1 \mathrm{H}, 10 '-\mathrm{H})$, $1.22-1.38\left(\mathrm{~m}, 1 \mathrm{H}, 9^{\prime}-\mathrm{H}^{\mathrm{B}}\right), 1.22-1.38\left(\mathrm{~m}, 1 \mathrm{H}, 11^{\prime}-\mathrm{H}^{\mathrm{B}}\right), 1.60\left(\mathrm{~d}, J_{3^{\prime \prime}, 2^{\prime \prime}}=13.0 \mathrm{~Hz}, 1 \mathrm{H}, 3^{\prime \prime}-\mathrm{H}^{\mathrm{A}}\right), 1.60$ $\left(\mathrm{d}, J_{5 "}^{\prime \prime} 6^{\prime \prime}=13.0 \mathrm{~Hz}, 1 \mathrm{H}, 5^{\prime \prime}-\mathrm{H}^{\mathrm{A}}\right), 1.73-1.81\left(\mathrm{~m}, 4 \mathrm{H}, 2^{\prime \prime}-\mathrm{H}_{2}, 6 "-\mathrm{H}_{2}\right), 1.85\left(\mathrm{~s}, 6{ }^{\prime}-\mathrm{CH}_{3}\right), 2.42(\mathrm{~m}, 1 \mathrm{H}$,

\footnotetext{
${ }^{18}$ Y. Cheng, B. Schneider, U. Riese, B. Schubert, Z. Li, M. Hamburger, J. Nat. Prod. 2006, 69, 436-438.
} 
3"-- $\left.{ }^{\mathrm{B}}\right), 2.42\left(\mathrm{~m}, 1 \mathrm{H}, 5^{\prime \prime}-\mathrm{H}^{\mathrm{B}}\right), 2.70\left(\mathrm{~m}_{\mathrm{c}}, 1 \mathrm{H}, 8^{\prime}-\mathrm{H}\right), 3.63\left(\mathrm{q}, J_{1^{\prime \prime}, 2^{\prime \prime}}=J_{1^{\prime \prime}, 6^{\prime \prime}}=6.3 \mathrm{~Hz}, 1 \mathrm{H}, 1^{\prime \prime}-\mathrm{H}\right), 5.56$ $\left(\mathrm{d}, J_{7^{\prime}, 8^{\prime}}=9.8 \mathrm{~Hz}, 1 \mathrm{H}, 7^{\prime}-\mathrm{H}\right), 6.46\left(\mathrm{dd}, J_{4^{\prime}, 3^{\prime}}=11.1 \mathrm{~Hz}, J_{4^{\prime}, 5^{\prime}}=14.9 \mathrm{~Hz}, 1 \mathrm{H}, 4^{\prime}-\mathrm{H}\right), 6.77(\mathrm{~d}$, $\left.J_{5^{\prime}, 4^{\prime}}=15.2 \mathrm{~Hz}, 1 \mathrm{H}, 5^{\prime}-\mathrm{H}\right), 7.64\left(\mathrm{dd}, J_{3^{\prime}, 4^{\prime}}=11.2 \mathrm{~Hz}, J_{3^{\prime}, 2^{\prime}}=14.6 \mathrm{~Hz}, 1 \mathrm{H}, 3{ }^{\prime}-\mathrm{H}\right), 7.96(\mathrm{~d}$, $\left.J_{2^{\prime}, 3^{\prime}}=14.9 \mathrm{~Hz}, 1 \mathrm{H}, 2^{\prime}-\mathrm{H}\right), 8.02(\mathrm{~s}, 1 \mathrm{H}, 6-\mathrm{H})$.

${ }^{13}$ C NMR (125.75 MHz, MeOD/MeOH, DsBrSe16-501103, 17.9.2019): $\delta=11.7$ (C-12'), 12.6 $\left(6^{\prime}-\mathrm{CH}_{3}\right), 19.5\left(10^{\prime}-\mathrm{CH}_{3}\right), 21.6\left(8^{\prime}-\mathrm{CH}_{3}\right), 31.2\left(\mathrm{C}-11^{\prime}\right), 31.5\left(\mathrm{C}-2^{\prime \prime}, \mathrm{C}-6 "\right), 32.0\left(\mathrm{C}-8^{\prime}\right), 33.7(\mathrm{C}-$ 10'), 34.7 (C-3", C-5"), 45.9 (C-9'), 70.7 (C-1"), 71.7 (C-4"), 107.5 (C-3), 119.6 (C-5), 126.4 (C-4'), 128.4 (C-2'), 134.2 (C-6'), 139.1 (C-6), 147.5 (C-7'), 147.7 (C-3'), 149.7 (C-5'), 160.1 (C-2), 174.8 (C-4), $195.4(\mathrm{C}-1 ')$.

edHSQC [,,short-range H,C-COSY“, 500.10 MHz/125.75 MHz, MeOD; $\delta\left({ }^{1} \mathrm{H}\right) \leftrightarrow \delta\left({ }^{13} \mathrm{C}\right): 0.85$ $\left(\mathrm{d}, 10^{\prime}-\mathrm{CH}_{3}\right) \leftrightarrow 19.5\left(10^{\prime}-\mathrm{CH}_{3}\right), 0.86\left(\mathrm{t}, 12^{\prime}-\mathrm{H}_{3}\right) \leftrightarrow 11.7\left(\mathrm{C}-12^{\prime}\right), 0.98\left(\mathrm{~d}, 8^{\prime}-\mathrm{CH}_{3}\right) \leftrightarrow 21.6\left(8^{\prime}-\right.$ $\left.\mathrm{CH}_{3}\right), 1.11-1.19\left(\mathrm{~m}, 9^{\prime}-\mathrm{H}^{\mathrm{A}}\right) \leftrightarrow 45.9\left(\mathrm{C}-9^{\prime}\right), 1.11-1.19\left(\mathrm{~m}, 11^{\prime}-\mathrm{H}^{\mathrm{A}}\right) \leftrightarrow 31.2\left(\mathrm{C}-11^{\prime}\right), 1.22-1.38(\mathrm{~m}$, $\left.10^{\prime}-\mathrm{H}\right) \leftrightarrow 33.7\left(\mathrm{C}-10^{\prime}\right), 1.22-1.38\left(\mathrm{~m}, 9^{\prime}-\mathrm{H}^{\mathrm{B}}\right) \leftrightarrow 45.9\left(\mathrm{C}-9^{\prime}\right), 1.22-1.38\left(\mathrm{~m}, 11^{\prime}-\mathrm{H}^{\mathrm{B}}\right) \leftrightarrow 31.2(\mathrm{C}-$ $\left.11^{\prime}\right), 1.60\left(\mathrm{~d}, 3 "-\mathrm{H}^{\mathrm{A}}\right) \leftrightarrow 34.7(\mathrm{C}-3 "), 1.60\left(\mathrm{~d}, 5^{\prime \prime}-\mathrm{H}^{\mathrm{A}}\right) \leftrightarrow 34.7(\mathrm{C}-5 "), 1.73-1.81\left(\mathrm{~m}, 2^{\prime \prime}-\mathrm{H}_{2}, 6^{\prime \prime}-\mathrm{H}_{2}\right)$ $\leftrightarrow 31.5\left(\mathrm{C}-2^{\prime \prime}, \mathrm{C}-6 "\right), 1.85\left(\mathrm{~s}, 6^{\prime}-\mathrm{CH}_{3}\right) \leftrightarrow 12.6\left(6^{\prime}-\mathrm{CH}_{3}\right), 2.42\left(\mathrm{~m}, 3^{\prime \prime}-\mathrm{H}^{\mathrm{B}}\right) \leftrightarrow 34.7\left(\mathrm{C}-3^{\prime \prime}\right), 2.42$ $\left(\mathrm{m}, 5^{\prime \prime}-\mathrm{H}^{\mathrm{B}}\right) \leftrightarrow 34.7\left(\mathrm{C}-5^{\prime \prime}\right), 2.70\left(\mathrm{~m}_{\mathrm{c}}, 8^{\prime}-\mathrm{H}\right) \leftrightarrow 32.0\left(\mathrm{C}-8^{\prime}\right), 3.63\left(\mathrm{q}, 1^{\prime \prime}-\mathrm{H}\right) \leftrightarrow 70.7\left(\mathrm{C}-1^{\prime \prime}\right), 5.56$ $\left(\mathrm{d}, 7^{\prime}-\mathrm{H}\right) \leftrightarrow 147.5\left(\mathrm{C}-7^{\prime}\right), 6.46\left(\mathrm{dd}, 4^{\prime}-\mathrm{H}\right) \leftrightarrow 126.4\left(\mathrm{C}-4^{\prime}\right), 6.77\left(\mathrm{~d}, 5^{\prime}-\mathrm{H}\right) \leftrightarrow 149.7\left(\mathrm{C}-5^{\prime}\right), 7.64$ $\left(\mathrm{dd}, 3^{\prime}-\mathrm{H}\right) \leftrightarrow 147.7\left(\mathrm{C}-3^{\prime}\right), 7.96\left(\mathrm{~d}, 2^{\prime}-\mathrm{H}\right) \leftrightarrow 128.4\left(\mathrm{C}-2^{\prime}\right), 8.02(\mathrm{~s}, 6-\mathrm{H}) \leftrightarrow 139.1$ (C-6).

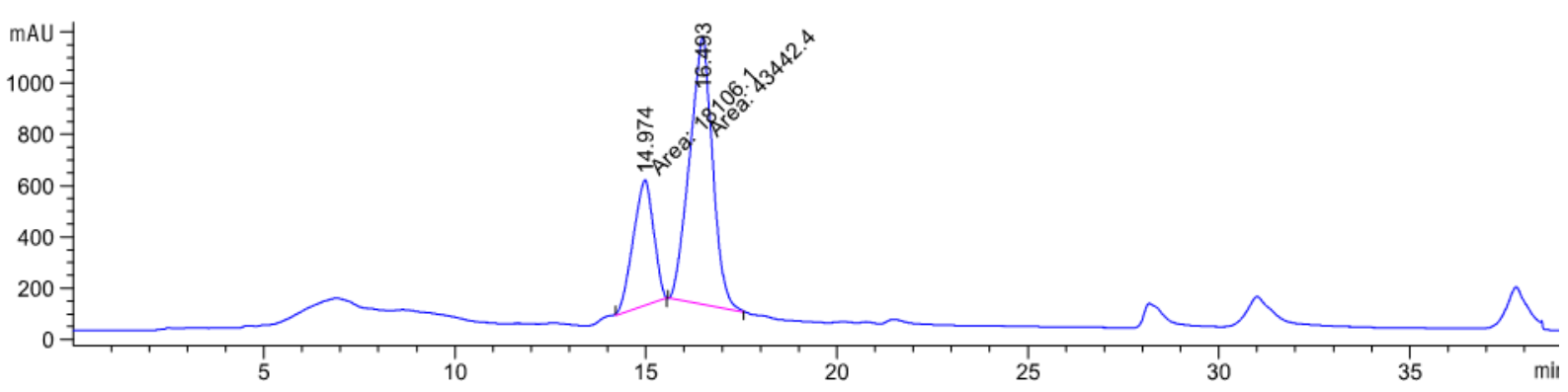
Isolation of deoxymilitarinone $A(4), t_{R}=14.9 \mathrm{~min}$ and militarinone $A(5), t_{R}=16.5 \mathrm{~min}(U V$-detection: $400 \mathrm{~nm})$.

HRMS (ESI) m/z: [M-H] ${ }^{+}$Calcd for $\mathrm{C}_{26} \mathrm{H}_{36} \mathrm{O}_{6} \mathrm{~N} 458.2548$; Found 458.2550 
and

$(4 S, 6 S, E)-2,4,6-T r i m e t h y l o c t-2-e n-1-o l ~[(S, S)-35]$

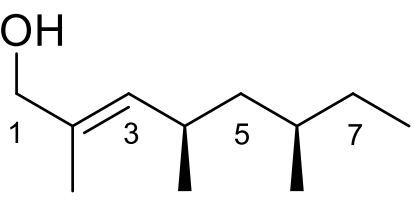

$(R, R)-35$

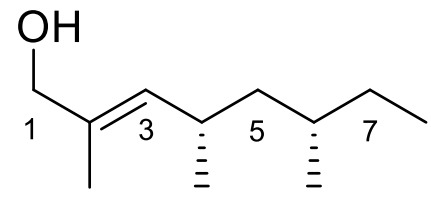

$(S, S)-35$

Method A: To a solution of the aldehyde $(R, R)-\mathbf{1 0}(31.8 \mathrm{mg}, 190 \mu \mathrm{mol})$ in $\mathrm{Et}_{2} \mathrm{O}(1.0 \mathrm{~mL})$ was added $\mathrm{LiAlH}_{4}(1 \mathrm{M}$ solution in THF, $250 \mu \mathrm{L}, 250 \mu \mathrm{mol}, 1.3$ eq. $)$ at $0^{\circ} \mathrm{C}$. The mixture was warmed to $25^{\circ} \mathrm{C}$ and held this temperature for $1.5 \mathrm{~h}$. The mixture was diluted with $\mathrm{H}_{2} \mathrm{O}(0.3 \mathrm{~mL})$ and $\mathrm{NaOH}$ (6 M, 3 drops). The mixture was filtered. The solvent was removed under reduced pressure and the residue was purified by flash chromatography (PE 30/50: $\mathrm{Et}_{2} \mathrm{O}=3: 1,1.5 \mathrm{~cm}$, $15 \mathrm{~cm}, 5 \mathrm{~mL})$. The alcohol $(R, R)-35(26.2 \mathrm{mg}, 79 \%, 160 \mu \mathrm{mol})$ was obtained as a colorless oil.

The enantiomer $(R, R)-35$ was obtained analogously in $80 \%$ yield.

Method B: Natural militarinone $\mathrm{C}[(S, R, R)-\mathbf{1}](12.0 \mathrm{mg}, 28.8 \mu \mathrm{mol})$ in THF: $\mathrm{H}_{2} \mathrm{O}(1: 1,3.8 \mathrm{~mL})$ was treated with $\mathrm{OsO}_{4} \cdot 2 \mathrm{H}_{2} \mathrm{O}\left(3.0 \mathrm{mg}, 10.6 \mu \mathrm{mol}, 0.37\right.$ eq.) and $\mathrm{NaIO}_{4}(86.4 \mathrm{mg}, 403 \mu \mathrm{mol}$, 14.0 eq.). The suspension stirred at $25^{\circ} \mathrm{C}$ for $1.5 \mathrm{~h}$. Then $\mathrm{OsO}_{4} \cdot 2 \mathrm{H}_{2} \mathrm{O}(1.5 \mathrm{mg}, 5.7 \mu \mathrm{mol}$, 0.20 eq.) and $\mathrm{NaIO}_{4}(61.1 \mathrm{mg}, 288 \mu \mathrm{mol}, 10.0$ eq. $)$ was added. After $1 \mathrm{~h}$ at $25^{\circ} \mathrm{C}$ it was added again $\mathrm{OsO}_{4} \cdot 2 \mathrm{H}_{2} \mathrm{O}$ (1.5 mg, $5.7 \mu \mathrm{mol}, 0.20$ eq.) and $\mathrm{NaIO}_{4}(61.1 \mathrm{mg}, 288 \mu \mathrm{mol}, 10.0$ eq.). The mixture stirred for $1 \mathrm{~h}$. The mixture cooled to $0^{\circ} \mathrm{C}$ and it was added $\mathrm{NaBH}_{4}(130 \mathrm{mg}, 3.46 \mathrm{mmol}$, 120 eq.). The mixture warmed to $25^{\circ} \mathrm{C}$ and held this temperature for $1.5 \mathrm{~h}$. The mixture was diluted with brine $(2 \mathrm{~mL})$ and $\mathrm{Et}_{2} \mathrm{O}(5 \mathrm{~mL})$. The layers were separated and the aq. layer was extracted with $\mathrm{Et}_{2} \mathrm{O}(3 \times 10 \mathrm{~mL})$. The combined organic layers were dried over $\mathrm{Na}_{2} \mathrm{SO}_{4}$ and the solvent was removed under reduced pressure. The residue was purified by flash chromatography $\left(\mathrm{PE} 30 / 50: \mathrm{Et}_{2} \mathrm{O}=3: 1,0.5 \mathrm{~cm}, 12 \mathrm{~cm}, 0.5 \mathrm{~mL}\right)$. The alcohol $(R, R)-35(1.0 \mathrm{mg}$, $20 \%, 5.8 \mu \mathrm{mol})$ was obtained as a colorless oil.

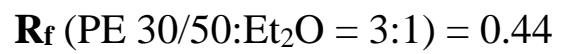

${ }^{1} \mathbf{H}$ NMR $\left(300.13 \mathrm{MHz}, \mathrm{CDCl}_{3} / \mathrm{CHCl}_{3}, \mathrm{DsBrOc} 15-30120,15.10 .2018\right): \delta=0.82\left(\mathrm{~d}, J_{6-}\right.$ $\left.\mathrm{CH}_{3}, 6=6.3 \mathrm{~Hz}, 3 \mathrm{H}, 6-\mathrm{CH}_{3}\right), 0.85\left(\mathrm{t}, J_{8,7}=7.1 \mathrm{~Hz}, 3 \mathrm{H}, 8-\mathrm{H}_{3}\right), 0.92\left(\mathrm{~d}, J_{4-\mathrm{CH}_{3}, 4}=6.7 \mathrm{~Hz}, 3 \mathrm{H}, 4-\right.$ 
Christian Drescher, Morris Keller, Olivier Potterat, Matthias Hamburger and Reinhard Brückner, Structure-Elucidating Total Synthesis of the (Polyenoyl)tetramic Acid Militarinone C

$\left.\mathrm{CH}_{3}\right), 1.02-1.16\left(\mathrm{~m}, 1 \mathrm{H}, 7-\mathrm{H}^{\mathrm{A}}\right), 1.02-1.16\left(\mathrm{~m}, 1 \mathrm{H}, 5-\mathrm{H}^{\mathrm{A}}\right), 1.19-1.32(\mathrm{~m}, 1 \mathrm{H}, 6-\mathrm{H}), 1.19-1.32$ $\left(\mathrm{m}, 1 \mathrm{H}, 7-\mathrm{H}^{\mathrm{B}}\right), 1.19-1.32\left(\mathrm{~m}, 1 \mathrm{H}, 5-\mathrm{H}^{\mathrm{B}}\right), 1.68\left(\mathrm{~d}, J_{2-\mathrm{CH}_{3}, 3}=1.4 \mathrm{~Hz}, 3 \mathrm{H}, 2-\mathrm{CH}_{3}\right), 2.49\left(\mathrm{~m}_{\mathrm{c}}, 1 \mathrm{H}\right.$, 4-H), $3.99\left(\mathrm{~d}, J_{1,1-\mathrm{OH}}=1.2 \mathrm{~Hz}, 2 \mathrm{H}, 1-\mathrm{H}_{2}\right), 5.13\left(\mathrm{dq}, J_{3,4}=9.6 \mathrm{~Hz},{ }^{4} J_{3,2-\mathrm{CH}_{3}}=1.3 \mathrm{~Hz}, 1 \mathrm{H}, 3-\mathrm{H}\right)$. 


\subsection{Chiral GLC Studies of the Enantiomeric Alcohol 35}
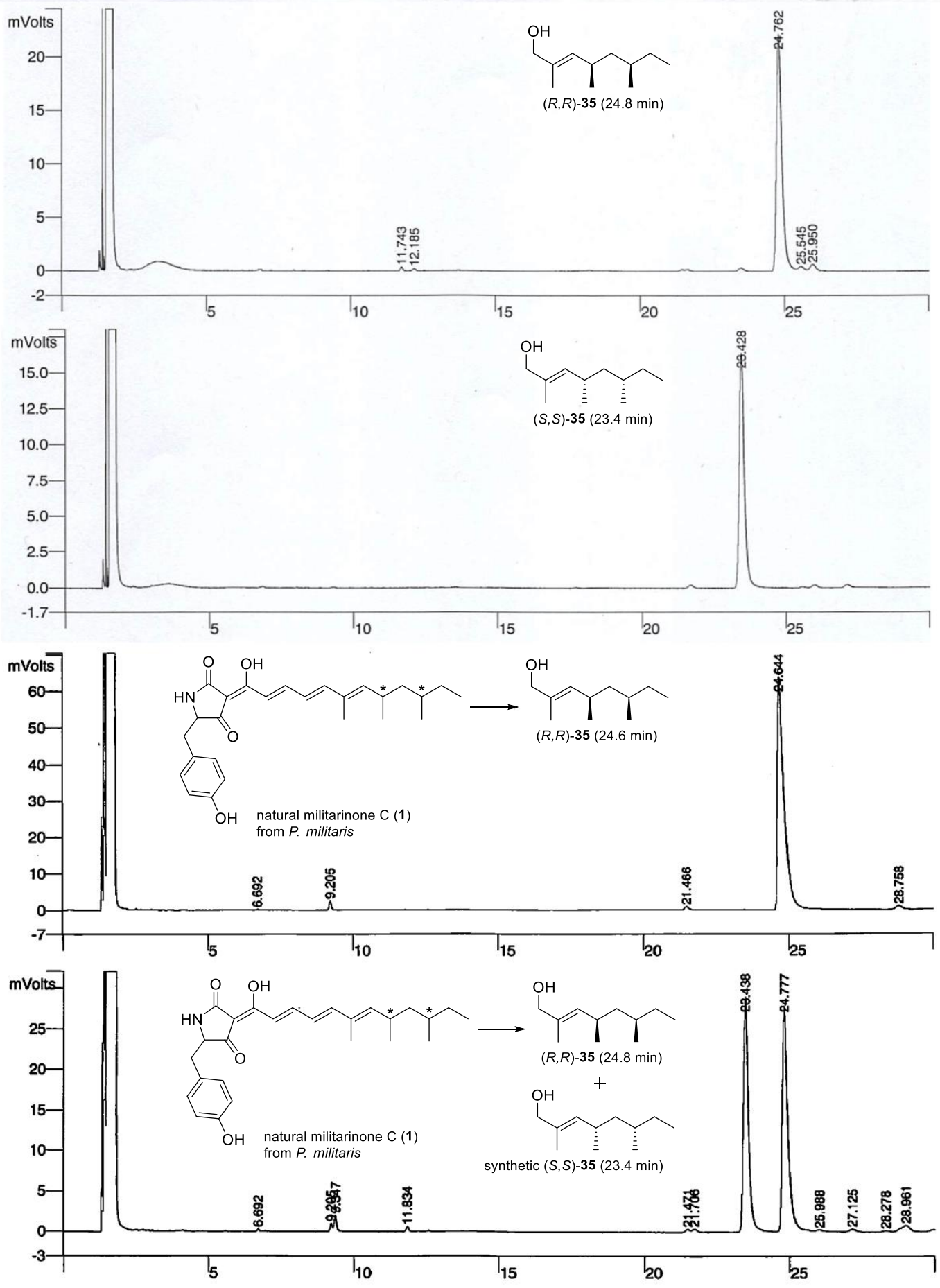
Christian Drescher, Morris Keller, Olivier Potterat, Matthias Hamburger and Reinhard Brückner, Structure-Elucidating Total Synthesis of the (Polyenoyl)tetramic Acid Militarinone C
Sample ID:
Drescher_TL-205-(R, R)
Operator (Calc):
Braukmueller

Injection Method: d:lbraukmuellerlmethodsldrescher_tl204_205_test_ma.mth Operator (Inj):

Data File: $\quad$ d:Ibraukmuellerldataldrescher_tl-205_03.09.19_17;46;06.run Channel FRONT INJEKTION:

Run Time (min): $\quad 29.970$

Instrument (Inj): $\quad$ Braukmueller

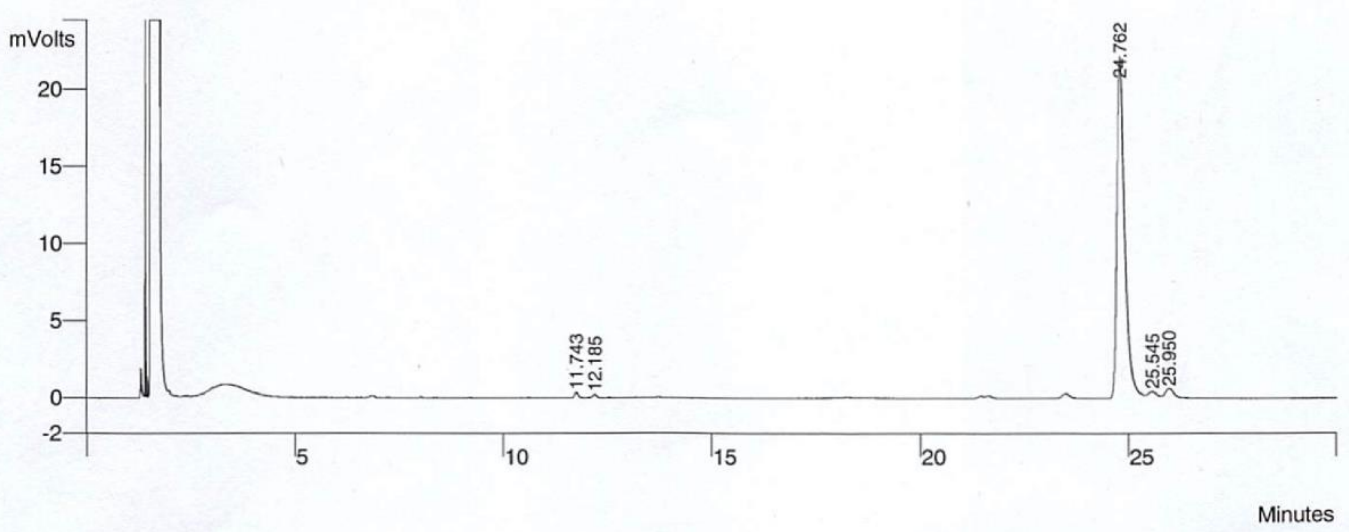

\begin{tabular}{cccccr} 
Peak Name & $\begin{array}{c}\text { Ret. Time } \\
\text { (min) }\end{array}$ & Result (\%) & Area (counts) & $\begin{array}{c}\text { Sep. } \\
\text { Code }\end{array}$ & $\begin{array}{c}\text { Peak Area } \\
\text { (counts) }\end{array}$ \\
\cline { 1 - 2 } & 11.743 & 0.7 & 2043 & BB & 2043 \\
12.185 & 0.4 & 1265 & BB & 1265 \\
24.762 & 95.9 & 296101 & BB & 296101 \\
25.545 & 0.9 & 2746 & BP & 2746 \\
25.950 & 2.1 & 6510 & PB & 6510
\end{tabular}

Method Notes

Varian $3800 \mathrm{GC}$ with Autosampler CP-8410

Front:
Agilent CYCLOSIL-B $30 \mathrm{~m}$ (Length), $0.250 \mathrm{~mm}$ (Diameter), $0.25 \mu \mathrm{m}$ (Film)

Erstinstallation 0222018

Ser-Nr.:

Agilent CP-ChiraSil-DEX CB $25 \mathrm{~m}$ (Length), $0.250 \mathrm{~mm}$ (Diameter), $0.25 \mu \mathrm{m}$ (Film)

Agilent CP-ChiraSil-

Ser.-Nr.: 9310258

Injektor (Front and Middle): $250^{\circ} \mathrm{C}$, Split-ratio: 100

Detektor (Front and Middle): $275^{\circ} \mathrm{C}$, FID

Column Oven: $100^{\circ} \mathrm{C}$ isotherma

Collumn Flow and Pressure:
Front: $10.0 \mathrm{psi},(1.4 \mathrm{~mL} / \mathrm{min})$, Linear Velocity $=44.4 \mathrm{~cm} / \mathrm{sec}$, Total-Flow $=148.2 \mathrm{~mL}$

Middle: $10.0 \mathrm{psi},(1.7 \mathrm{~mL} / \mathrm{min})$. Linear Velocity $=53.3 \mathrm{~cm} / \mathrm{sec}$, Total-Flow $=176.8 \mathrm{~mL}$

Injection Notes: $\quad 100^{\circ} \mathrm{C}$ isotherm, front $10 \mathrm{psi}, \mathrm{Split}=100,8 \mu \mathrm{L}$, front only

Calculation Notes: 
Christian Drescher, Morris Keller, Olivier Potterat, Matthias Hamburger and Reinhard Brückner, Structure-Elucidating Total Synthesis of the (Polyenoyl)tetramic Acid Militarinone C

Sample ID: $\quad$ Drescher_TL-204 - $(S, S) \quad$ Operator (Calc): Braukmueller

Injection Method: d:Ibraukmuellerlmethodsldrescher_t1204_205_test_ma.mth Operator (Inj):

Data File: d:lbraukmuellerldataldrescher_tl-204_03.09.19_17;14;18.run

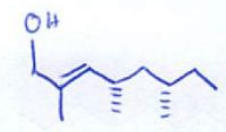

Channel FRONT INJEKTION:

Run Time (min): $\quad 29.970$

Instrument (Inj): $\quad$ Braukmueller

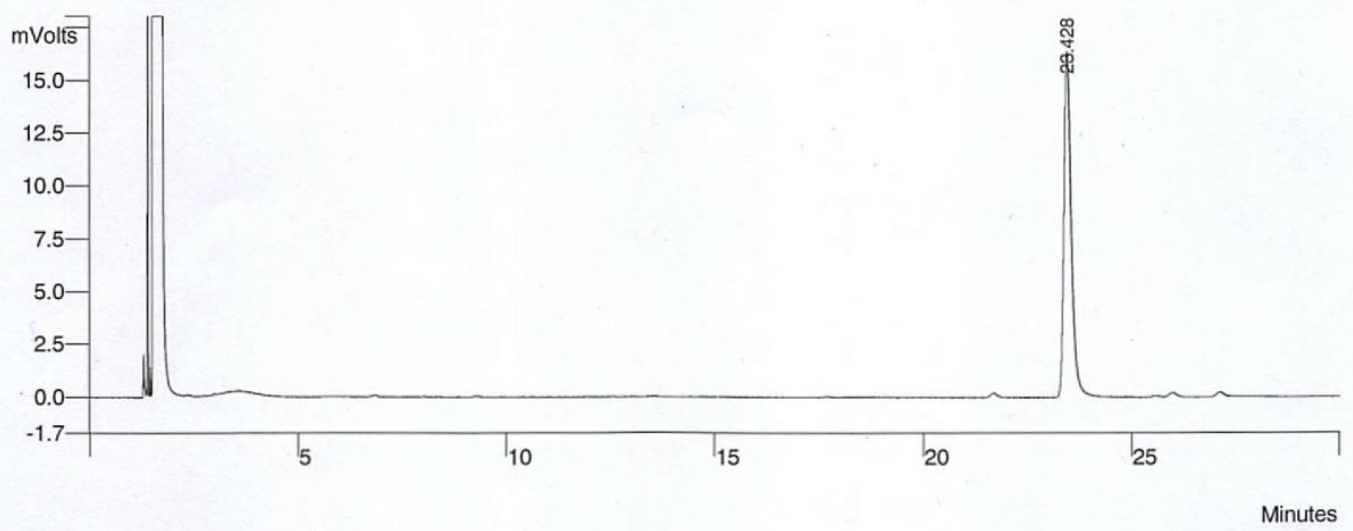

\begin{tabular}{|c|c|c|c|c|c|}
\hline Peak Name & $\underset{\text { (min) }}{\text { Ret. Time }}$ & Result (\%) & Area (counts) & $\begin{array}{l}\text { Sep. } \\
\text { Code }\end{array}$ & $\begin{array}{c}\text { Peak Area } \\
\text { (counts) }\end{array}$ \\
\hline & 23.428 & 100.0 & 198691 & BB & 198691 \\
\hline
\end{tabular}

Method Notes

with Autosampler CP-8410

V.

Erstinstallation 022018

Ser-Nr: USD681842H

Middle:

Agilent CP-ChiraSil-DEX CB $25 \mathrm{~m}$ (Length), $0.250 \mathrm{~mm}$ (Diameter), $0.25 \mu \mathrm{m}$ (Film)

Erstinstallation $02 / 2018$

Ser-Nr.: 9310258

Injektor (Front and Middle): $250^{\circ} \mathrm{C}$, Split-ratio: 100

Detektor (Front and Middle): $275^{\circ} \mathrm{C}$, FID

Column Oven: $100^{\circ} \mathrm{C}$ isothermal
Collumn Flow and Pressure:

Front: $10.0 \mathrm{psi}$, (1.4 mL

Mront: $10.0 \mathrm{psi},(1.4 \mathrm{~mL} / \mathrm{min})$. Linear Velocity $=44.4 \mathrm{~cm} / \mathrm{sec}$, Total-Flow $=148.2 \mathrm{~mL}$

Injection Notes: $\quad 100^{\circ} \mathrm{C}$ isotherm, front $10 \mathrm{psi}, \mathrm{Split}=100,8 \mu \mathrm{L}$, front only

Calculation Notes: 
Christian Drescher, Morris Keller, Olivier Potterat, Matthias Hamburger and Reinhard Brückner, Structure-Elucidating Total Synthesis of the (Polyenoyl)tetramic Acid Militarinone C

Sample ID: Drescher_CD-extract Operator (Calc): Braukmueller

Injection Method: d:Ibraukmuellertmethodsldrescher_t204_205_split_ma.mth

Operator (Inj):

Data File: · d:lbraukmuellerldataldrescher_cd-extract_03.09.19_15;40;30.run

Channel FRONT INJEKTION:

Run Time (min): $\quad 29.970$

Instrument (Inj): $\quad$ Braukmueller

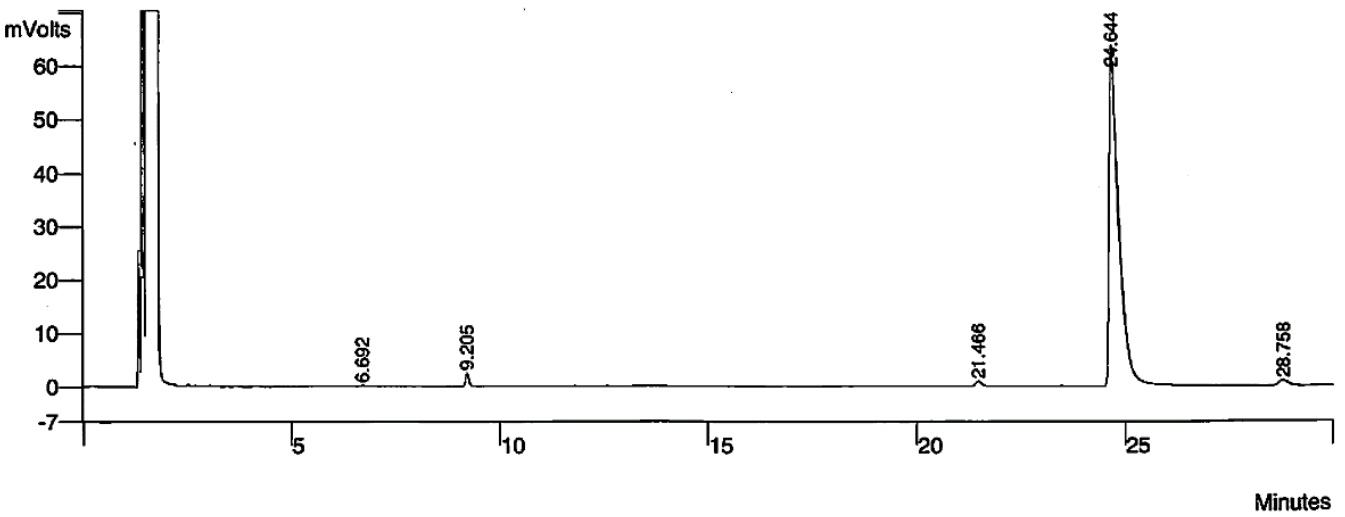

\begin{tabular}{cccccr} 
Peak Name & $\begin{array}{c}\text { Ret. Time } \\
\text { (min) }\end{array}$ & Result (\%) & Area (counts) & $\begin{array}{c}\text { Sep. } \\
\text { Code }\end{array}$ & $\begin{array}{c}\text { Peak Area } \\
\text { (counts) }\end{array}$ \\
\hline & & & & & \\
\hline 9.692 & 0.1 & 1050 & BB & 1050 \\
21.465 & 1.3 & 14183 & BB & 14183 \\
24.644 & 96.1 & 10676 & BB & 10676 \\
28.758 & 1.5 & 1051405 & BB & 1051405 \\
& & 16441 & BB & 16441
\end{tabular}

Mathod Notes

Varian $3800 \mathrm{GC}$ with Autosampler CP-8410

Front:

Erstinstallation 02/2018

Injektor (Front and Middle): $250^{\circ} \mathrm{C}$. Split-ratio: 25

Detektor (Front and Middle): $275^{\circ} \mathrm{C}$, FD

Column Oven: $100^{\circ} \mathrm{C}$ isotherm

Front: 10.0 psi., (1.4 mL/min), Linear Velocity $=44.4 \mathrm{~cm} / \mathrm{sec}$, Total - Flow $=76.8 \mathrm{~mL}$

Injection Notes:

$100^{\circ} \mathrm{C}$ isotherm, front $10 \mathrm{psi}, \mathrm{Split}=25,4 \mu \mathrm{L}$, front only

Calculation Notes: 
Christian Drescher, Morris Keller, Olivier Potterat, Matthias Hamburger and Reinhard Brückner, Structure-Elucidating Total Synthesis of the (Polyenoyl)tetramic Acid Militarinone C

Sample ID: $\quad$ CD-178-SS+CDextract $\quad$ Operator (Calc): $\quad$ Braukmueller

Injection Method: d:Ibraukmuellerlmethodsldrescher_t1204_205_split_ma.mth

Operator (Inj):

Data File: $\quad$ d:Ibraukmuellerldatalcd-178-ss+cdextract_03.09.19_16;12;16.run

Channel FRONT INJEKTION:

Run Time (min): $\quad 29.970$

Instrument (Inj): $\quad$ Braukmueller

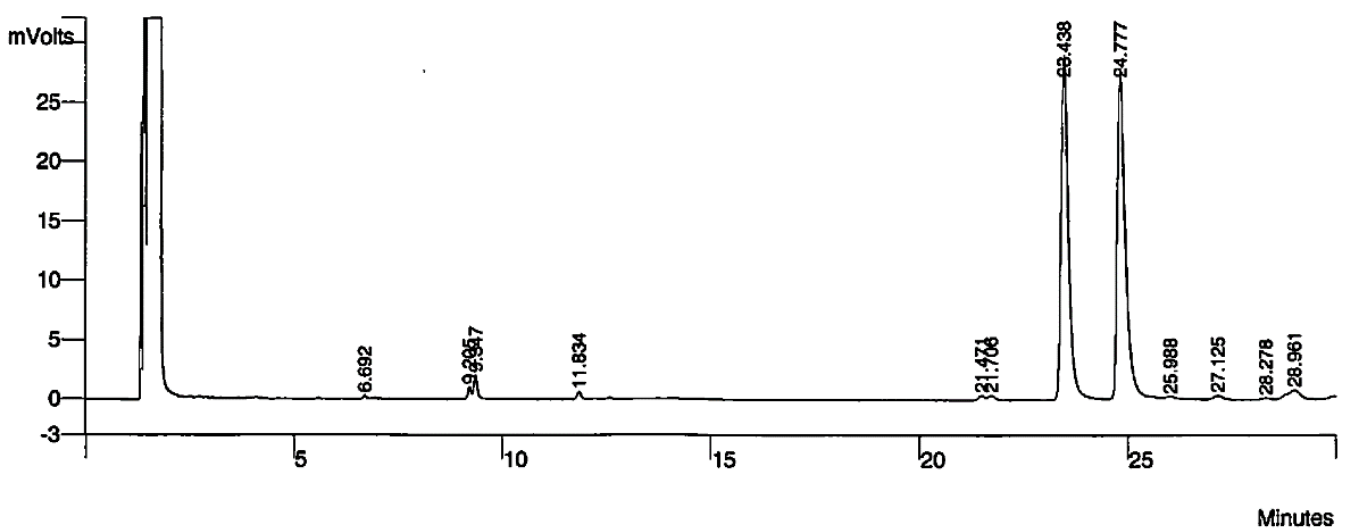

\begin{tabular}{cccccr} 
Peak Name & $\begin{array}{c}\text { Ret. Time } \\
\text { (min) }\end{array}$ & Result (\%) & Area (counts) & $\begin{array}{c}\text { Sep. } \\
\text { Code }\end{array}$ & $\begin{array}{c}\text { Peak Area } \\
\text { (counts) }\end{array}$ \\
\hline 6.692 & 0.2 & 1301 & BB & 1301 \\
& 0.7 & 5340 & BV & 5340 \\
9.347 & 1.5 & 11891 & VB & 11891 \\
11.834 & 0.5 & 4066 & BB & 4066 \\
21.471 & 0.4 & 3602 & BV & 3602 \\
21.706 & 0.5 & 4142 & VB & 4142 \\
23.438 & 44.5 & 360705 & BB & 360705 \\
24.777 & 48.6 & 393776 & BB & 393776 \\
25.988 & 0.2 & 2009 & BB & 2009 \\
27.125 & 0.6 & 4947 & BB & 4947 \\
28.278 & 0.2 & 1568 & BV & 1568 \\
28.961 & 2.2 & 17437 & VB & 17437
\end{tabular}

\section{Method Notes}

Varian 3800 GC with Autosampler CP-8410

Front:

Agilent CYCLOSIL-B $30 \mathrm{~m}$ (Length), $0.250 \mathrm{~mm}$ (Diameter), $0.25 \mu \mathrm{m}$ (Film)

Erstinstallation 0222018

Ser.-Nr.: USD681842H

Injektor (Front and Middle): $250^{\circ} \mathrm{C}$, Split-ratio: 25

Detektor (Front and Middle): $275^{\circ} \mathrm{C}$. FID

Column Oven: $100^{\circ} \mathrm{C}$ isothermen

Front: $10.0 \mathrm{psi},(1.4 \mathrm{~mL} / \mathrm{min})$, Linear Velociry a $44.4 \mathrm{~cm} / \mathrm{sec}$, Total-Flow $=76.8 \mathrm{~mL}$

Injection Notes:

$100^{\circ} \mathrm{C}$ isotherm, front $10 \mathrm{psi}$, Split $=25,4 \mu \mathrm{L}$, front only

Calculation Notes: 


\subsection{HPLC-Report of the DMB-Tyrosine Ester 13 and the Western}

\section{Block 8}

\section{HPLC-report of the DMB-tyrosine ester 13.}

\section{D-7000 HPLC System Manager Report}

Analyzed: 06/06/18 05:31 PM

Reported: 06/06/18 05:47 PM

Data Path: D: $\backslash$ Aquisition $\backslash$ Drescher $\backslash D A T A \backslash 0002 \backslash$ Processed: 06/06/18 05:47 PM Processing Method: Drescher_TL-170

System(acquisition): Sys No. 9 Application: Drescher

Series : 0002

Sample Name: Drescher_TL-170-20-30-rac

Vial Number:

Injection from this vial: 1 of 1

Vial Type: UNK

Sample Description: nHept/EtOH95:5,0.8mL,229nm, RT

Absorbance Mode: NORMAL (2.0 AU)

Spectral Bandwidth: AUTO

Absorbance Scale: Auto Spectral Interval: $200 \mathrm{~ms}$

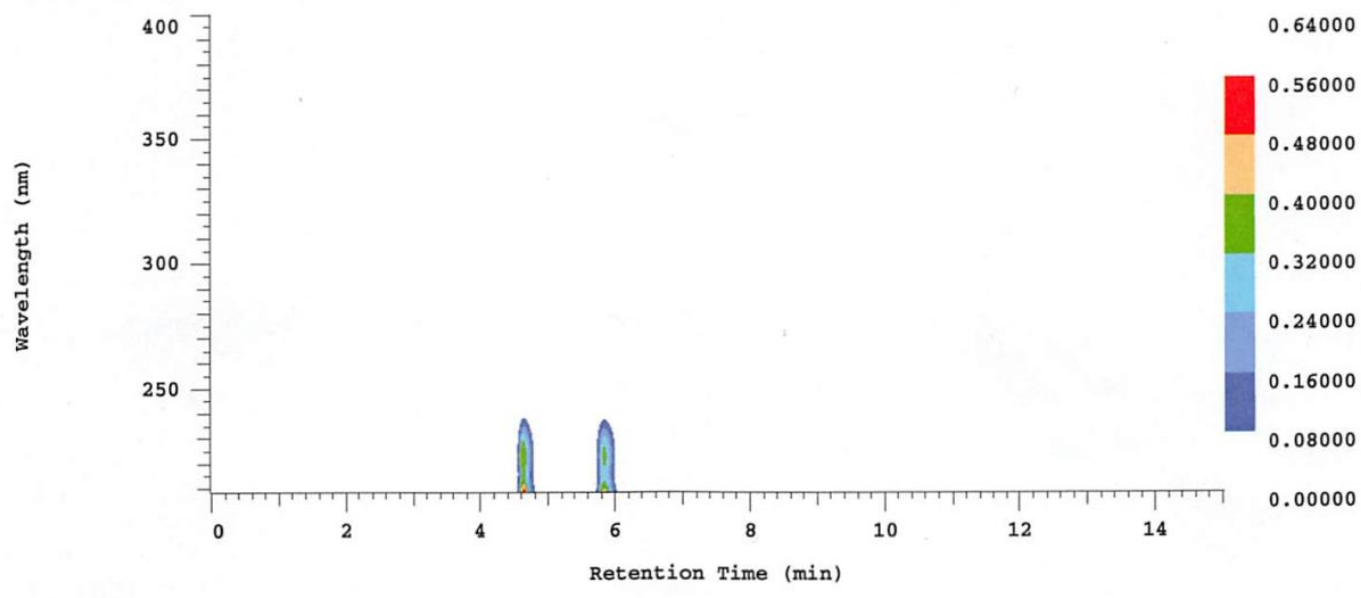

Chrom Type: Fixed WL Chromatogram, 229 nm

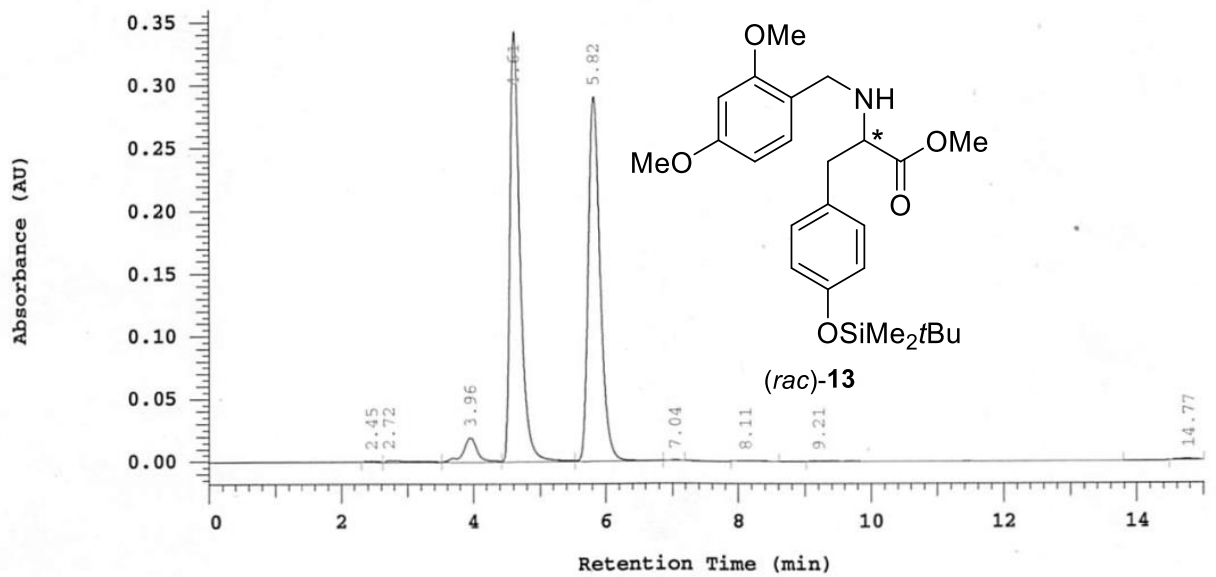

Acquisition Method: Drescher_TL-170

Column Type: Kromasil 3-CelluCoat 
Christian Drescher, Morris Keller, Olivier Potterat, Matthias Hamburger and Reinhard Brückner, Structure-Elucidating Total Synthesis of the (Polyenoyl)tetramic Acid Militarinone C

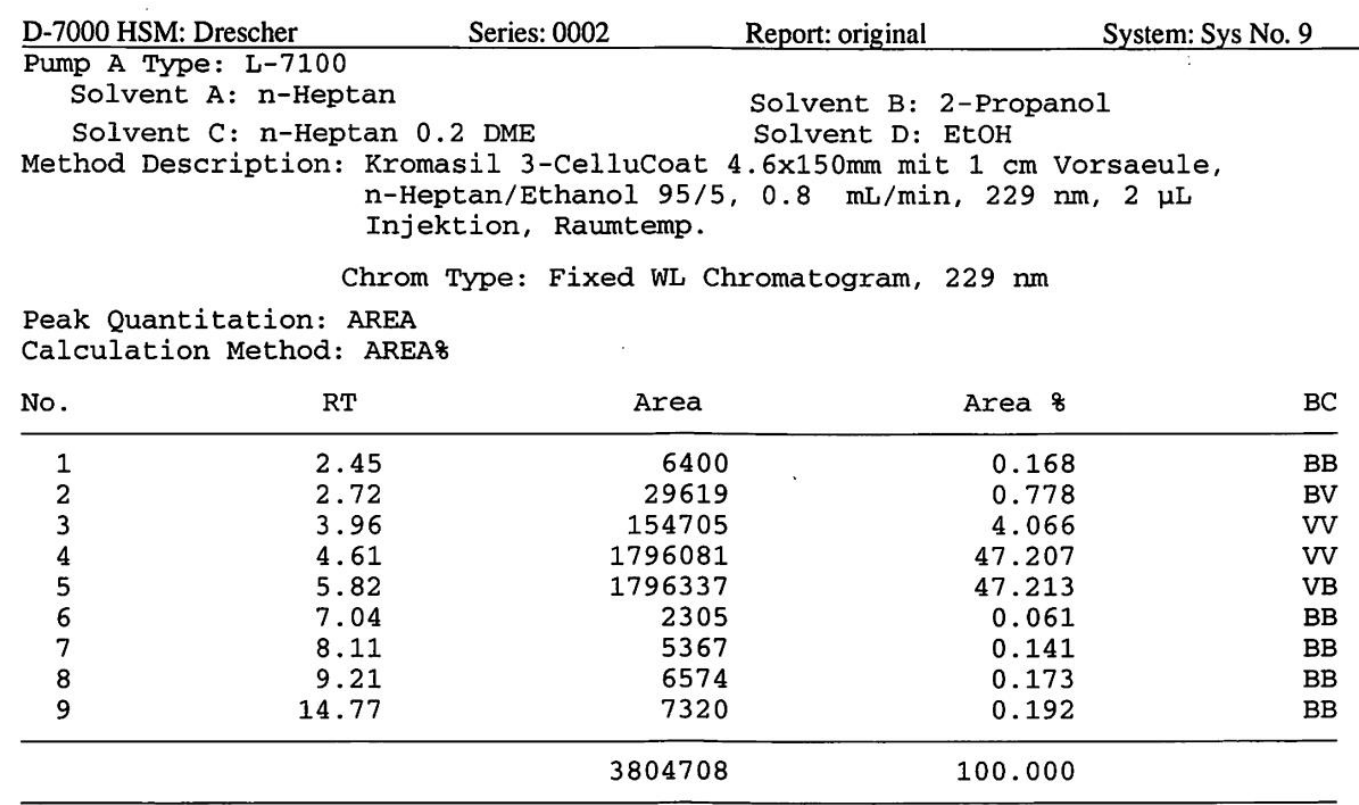

Peak rejection level: 0 


\section{D-7000 HPLC System Manager Report}

Analyzed: 07/16/18 11:26 AM

Data Path: D: \Aquisition \Drescher $\backslash$ DATA $\backslash 0013 \backslash$

Processing Method: Drescher_TL-170

System(acquisition): Sys No. 9

Application: Drescher

Sample Name: Drescher CD-185-2_ee

Injection from this vial: 1 of 1

Sample Description: nHept/EtOH95:

Absorbance Mode: NORMAL (2.0 AU)

Spectral Bandwidth: AUTO

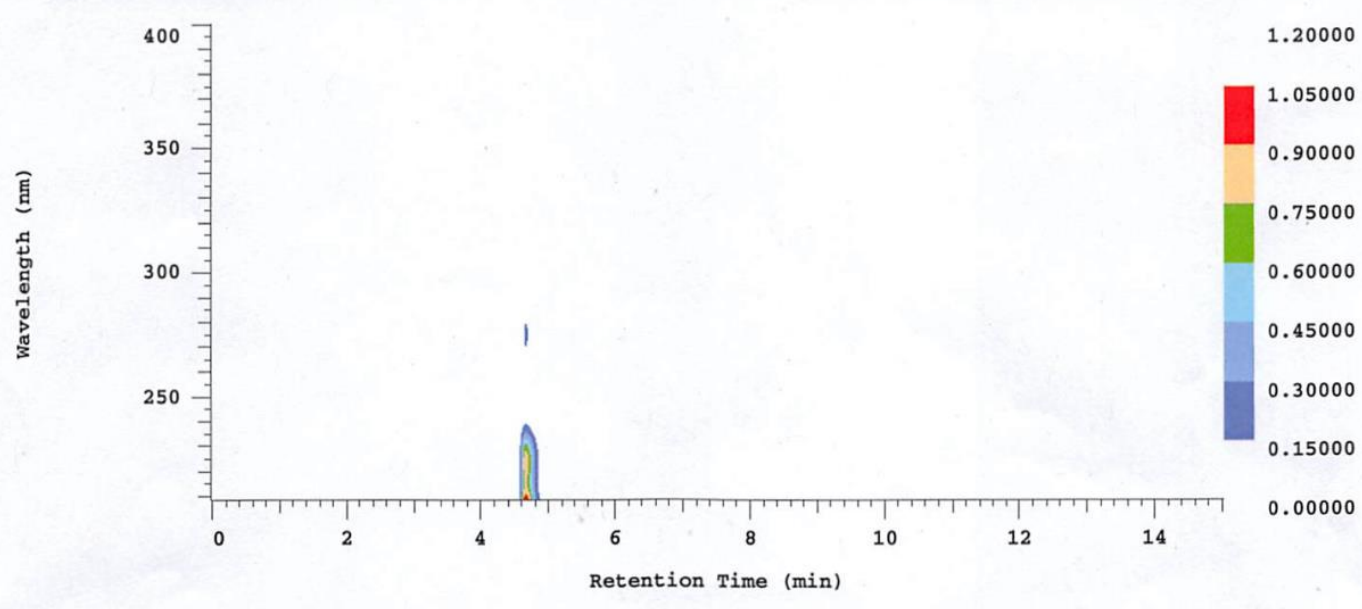

Chrom Type: Fixed WL Chromatogram, 229 nm

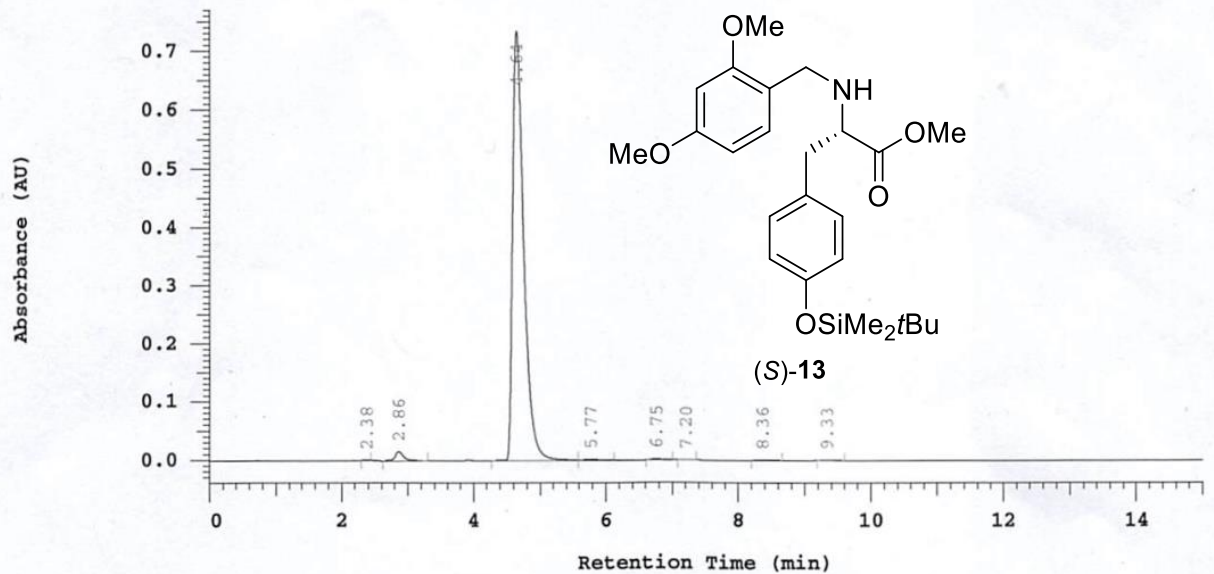

Acquisition Method: Drescher_TL-170

Column Type: Kromasil 3-CelluCoat

$$
4.6 \times 150 \mathrm{~mm}
$$

Reported: 07/16/18 11:41 AM Processed: 07/16/18 11:41 AM

Series : 0013

Vial Number: 1

Vial Type: UNK

Absorbance Scale: Auto

Spectral Interval: $200 \mathrm{~ms}$

.20000

.9000

.75000

60000

30000

15000

.00000
Volume: $2.0 \mathrm{ul}$ 
Christian Drescher, Morris Keller, Olivier Potterat, Matthias Hamburger and Reinhard Brückner, Structure-Elucidating Total Synthesis of the (Polyenoyl)tetramic Acid Militarinone C

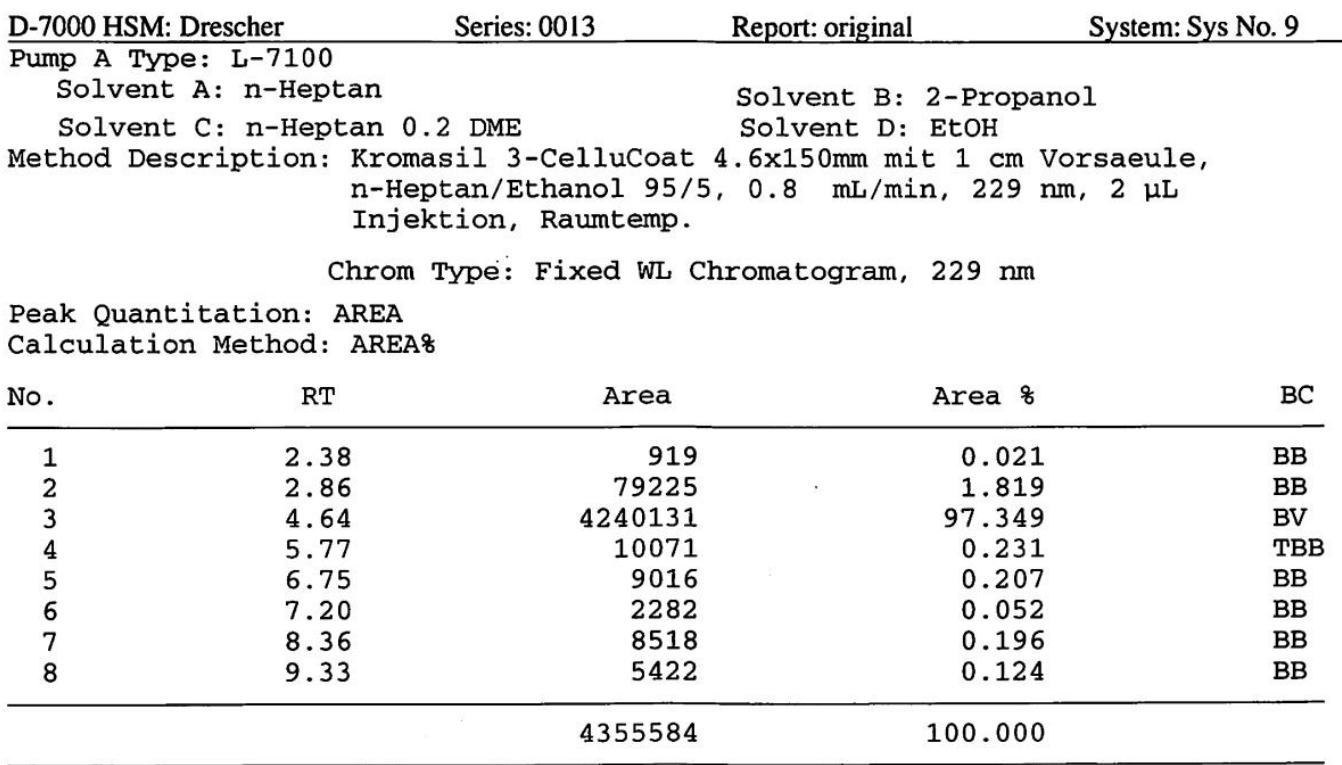

Peak rejection level: 0 


\section{5}

\section{D-7000 HPLC System Manager Report}

Analyzed: 12/05/19 06:24 PM

Reported: 12/05/19 06:39 PM

Processed: 12/05/19 06:39 PM

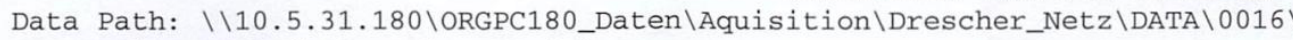
Processing Method: Drescher_TL-170

System(acquisition): Sys No. 9

Series: 0016

Application: Drescher_Netz

Sample Name: Drescher_CD-185-S_ee

Injection from this vial: 1 of 1

Sample Description: nHept/EtOH95:5,0.8mL,229nm, R

Absorbance Mode: NORMAL (2.0 AU)

Spectral Bandwidth: AUTO

Absorbance Scale: Auto

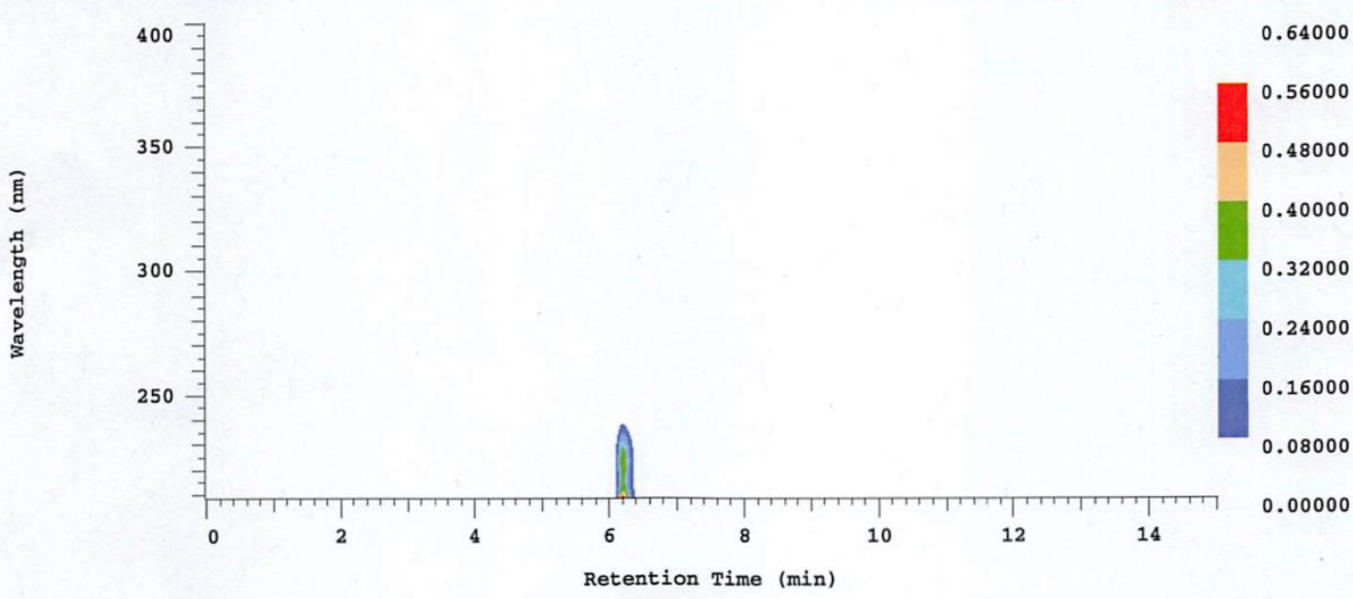

Chrom Type: Fixed WL Chromatogram, 229 nm

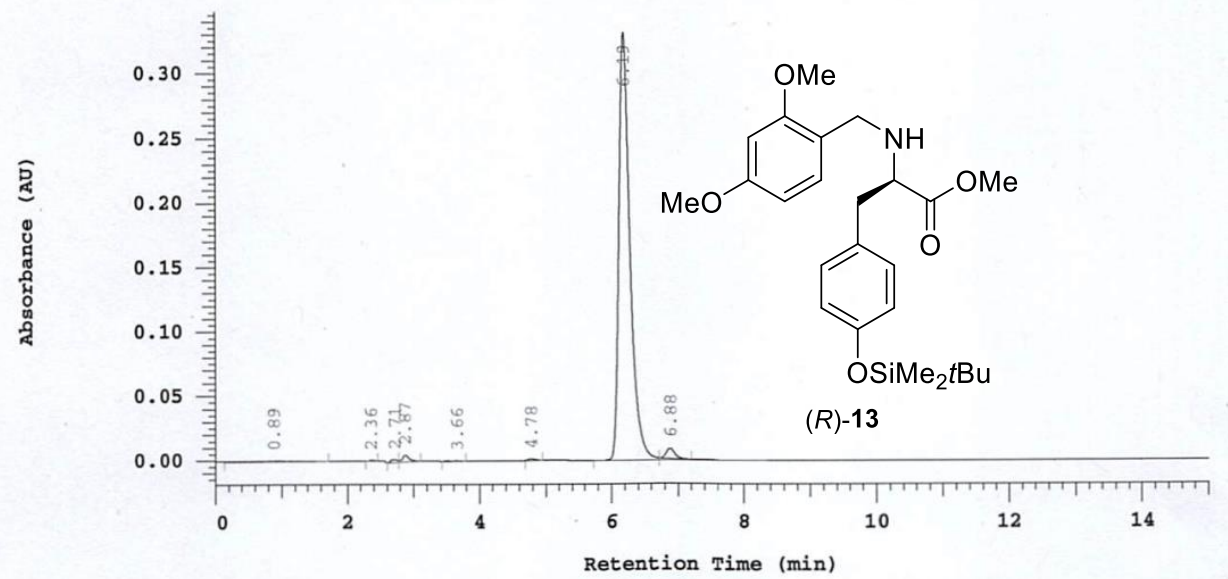

Acquisition Method: Drescher_TL-170

Column Type: Kromasil 3-CelluCoat

Developed by: Braukmueller

$4.6 \times 150 \mathrm{~mm}$

Page Indicator 
Christian Drescher, Morris Keller, Olivier Potterat, Matthias Hamburger and Reinhard Brückner, Structure-Elucidating Total Synthesis of the (Polyenoyl)tetramic Acid Militarinone C

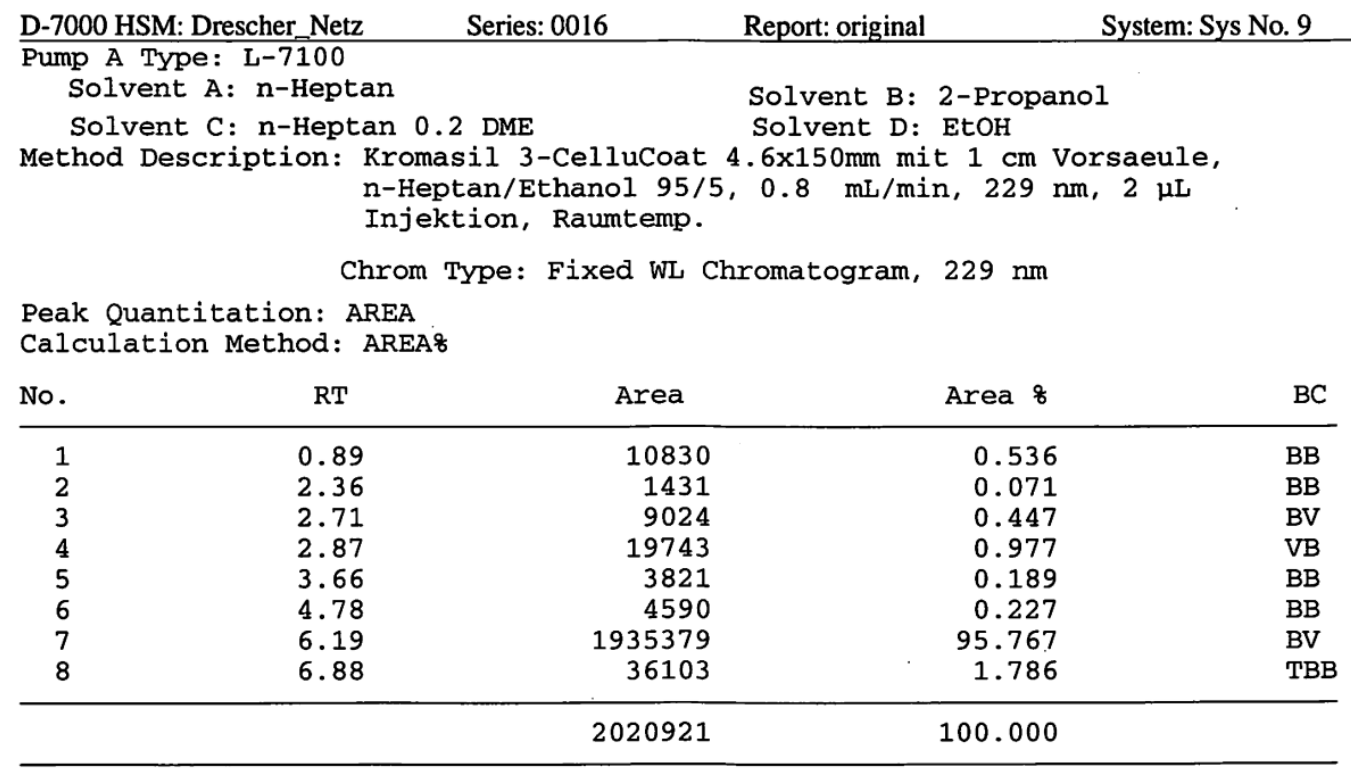

Peak rejection level: 0 
Christian Drescher, Morris Keller, Olivier Potterat, Matthias Hamburger and Reinhard Brückner, Structure-Elucidating Total Synthesis of the (Polyenoyl)tetramic Acid Militarinone C

HPLC-report of the Western Block 8.

Data File D: ILC_DATA IDRESCHER \DATA IDRESCHER_TL-166_RAC_2018-05-1714-42-22SYSTEM.D

Sample Name: Drescher_TL-166_rac_
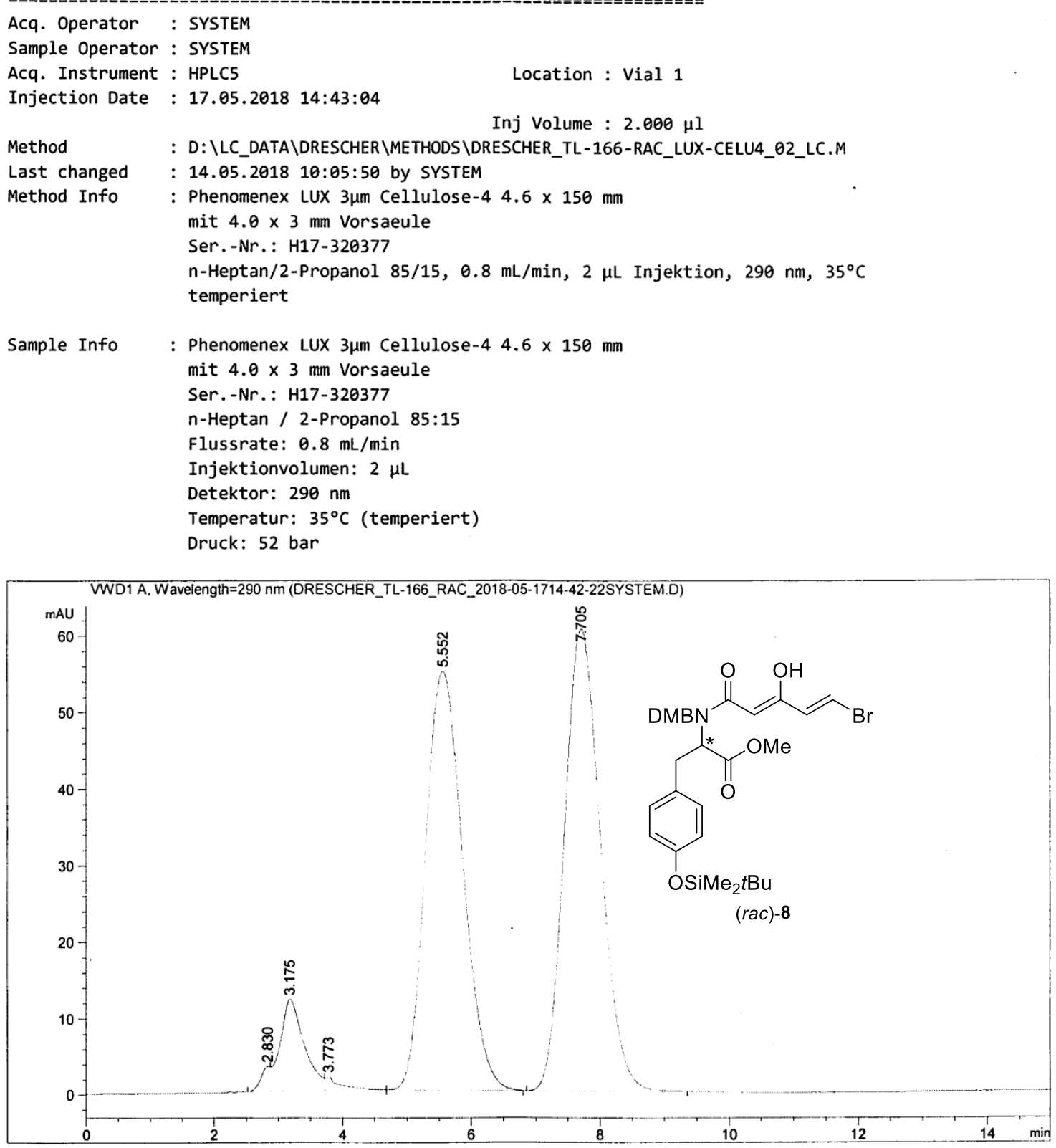
Christian Drescher, Morris Keller, Olivier Potterat, Matthias Hamburger and Reinhard Brückner, Structure-Elucidating Total Synthesis of the (Polyenoyl)tetramic Acid Militarinone C

Data File D: \LC_DATA \DRESCHER \DATA \DRESCHER_TL-166_RAC_2018-05-1714-42-22SYSTEM.D Sample Name: Drescher_TL-166_rac_

\begin{tabular}{|c|c|c|}
\hline \multicolumn{3}{|c|}{ Area Percent Report } \\
\hline 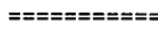 & $==x==x========0$ & 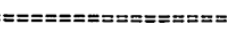 \\
\hline Sorted By & Signal & \\
\hline Multiplier & 1.0000 & \\
\hline Dilution & 1.0000 & $\cdot$ \\
\hline
\end{tabular}

Signal 1: VWD1 A, Wavelength $=290 \mathrm{~nm}$

\begin{tabular}{|c|c|c|c|c|c|c|}
\hline $\begin{array}{c}\text { Peak } \\
\quad \#\end{array}$ & $\begin{array}{c}\text { RetTime } \\
\text { [min] }\end{array}$ & Type & $\begin{array}{l}\text { Width } \\
\text { [min] }\end{array}$ & $\begin{array}{c}\text { Area } \\
{\left[\mathrm{mAU}^{*} \mathrm{~s}\right]}\end{array}$ & $\begin{array}{l}\text { Height } \\
\text { [mAU] }\end{array}$ & $\begin{array}{c}\text { Area } \\
\%\end{array}$ \\
\hline---1 & 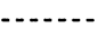 & & & | & $6-2-0-1$ & 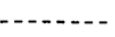 \\
\hline 1 & 2.830 & BV & 0.1521 & 33.48678 & 3.29279 & 0.6767 \\
\hline 2 & 3.175 & w & 0.3670 & 311.13867 & 12.03973 & 6.2879 \\
\hline 3 & 3.773 & VB & 0.2026 & 29.99809 & 1.89060 & 0.6062 \\
\hline 4 & 5.552 & BB & 0.6378 & 2221.90723 & 54.77637 & 44.9032 \\
\hline 5 & 7.705 & BB & 0.6085 & 2351.67993 & 60.41617 & 47.5259 \\
\hline Tota & & & & 4948.21071 & 132.41567 & \\
\hline
\end{tabular}

*** End of Report *** 
Christian Drescher, Morris Keller, Olivier Potterat, Matthias Hamburger and Reinhard Brückner, Structure-Elucidating Total Synthesis of the (Polyenoyl)tetramic Acid Militarinone C

Data Fille D: \LC_DATA \DRESCHER \DATA \DRESCHER_CD-WEST-S_EE_2018-08-1618-31-42SYSTEM.D

Sample Name: Drescher_CD-West-S_ee_
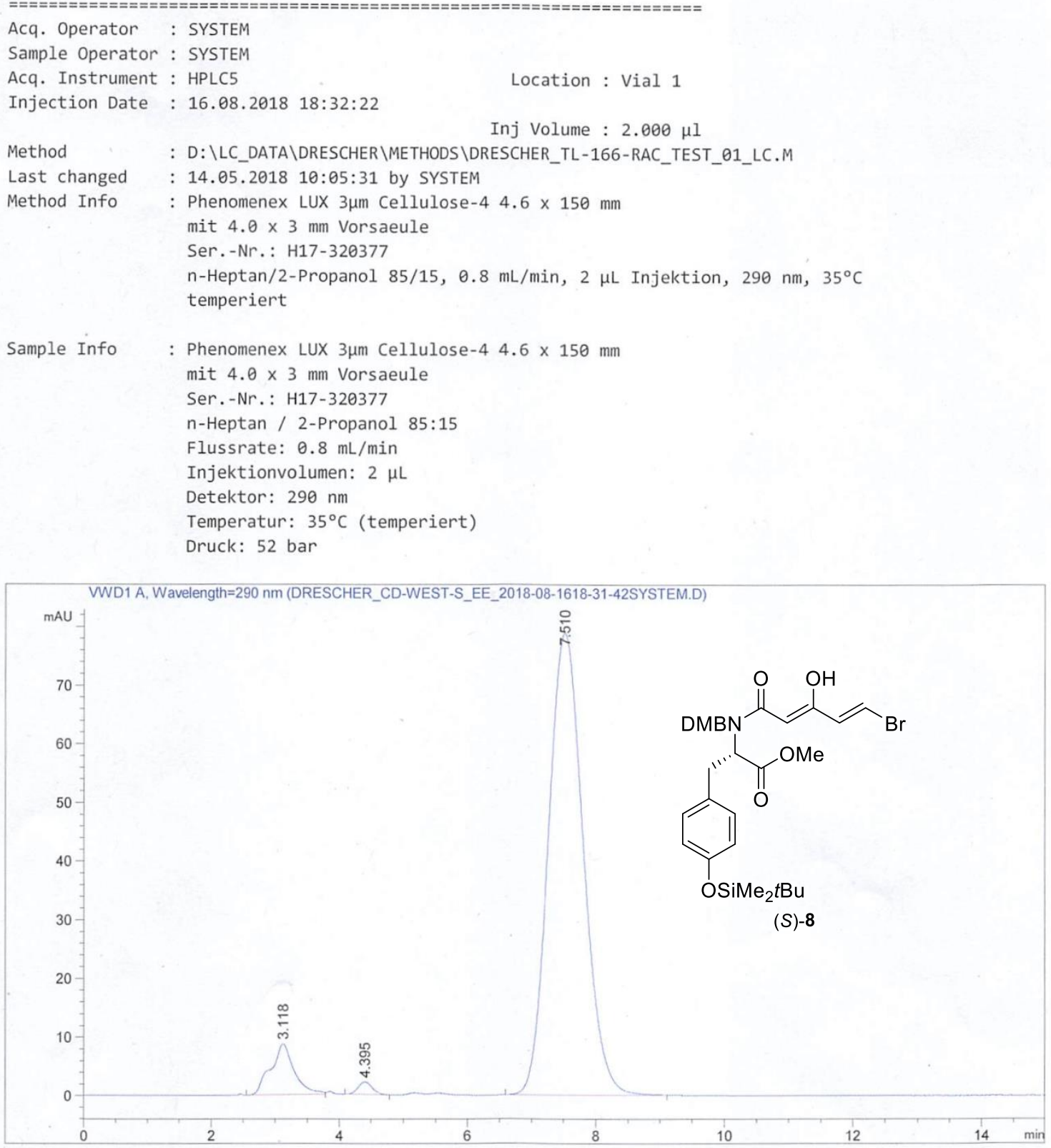
Christian Drescher, Morris Keller, Olivier Potterat, Matthias Hamburger and Reinhard Brückner, Structure-Elucidating Total Synthesis of the (Polyenoyl)tetramic Acid Militarinone C

Dáta File D: \LC_DATA IDRESCHER \DATA DRESCHER_CD-WEST-S_EE_2018-08-1618-31-42SYSTEM.D Sample Name: Drescher_CD-West-S_ee_

Area Percent Report

\begin{tabular}{|c|c|c|}
\hline Sorted By & $:$ & Signal \\
\hline Multiplier & : & 1.0000 \\
\hline Dilution & : & 1.0000 \\
\hline
\end{tabular}

Use Multiplier \& Dilution Factor with ISTDs

Signal 1: VWD1 A, Wavelength $=290 \mathrm{~nm}$

\begin{tabular}{|c|c|c|c|c|c|c|}
\hline $\begin{array}{c}\text { Peak } \\
\#\end{array}$ & $\begin{array}{c}\text { RetTime } \\
\text { [min] }\end{array}$ & Type & $\begin{array}{l}\text { Width } \\
\text { [min] }\end{array}$ & $\begin{array}{c}\text { Area } \\
{\left[\mathrm{mAU}^{*} \mathrm{~s}\right]}\end{array}$ & $\begin{array}{l}\text { Height } \\
{[\mathrm{mAU}]}\end{array}$ & $\begin{array}{c}\text { Area } \\
\%\end{array}$ \\
\hline & & & & 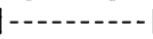 & & \\
\hline 1 & 3.118 & BV & 0.3496 & 223.66139 & 8.73809 & 6.8968 \\
\hline 2 & 4.395 & BB & 0.2422 & 33.51468 & 2.17689 & 1.0335 \\
\hline 3 & 7.510 & BB & 0.5917 & 2985.81372 & 78.86128 & 92.0698 \\
\hline Tota] & Is : & & & 3242.98980 & 89.77626 & \\
\hline
\end{tabular}

*** End of Report *** 
Christian Drescher, Morris Keller, Olivier Potterat, Matthias Hamburger and Reinhard Brückner, Structure-Elucidating Total Synthesis of the (Polyenoyl)tetramic Acid Militarinone C

Dáta File D: \LC_DATA \DRESCHER\DATA \DRESCHER_CD-WEST-R_EE_2018-08-1619-00-00SYSTEM.D Sample Name: Drescher_CD-West-R_ee_
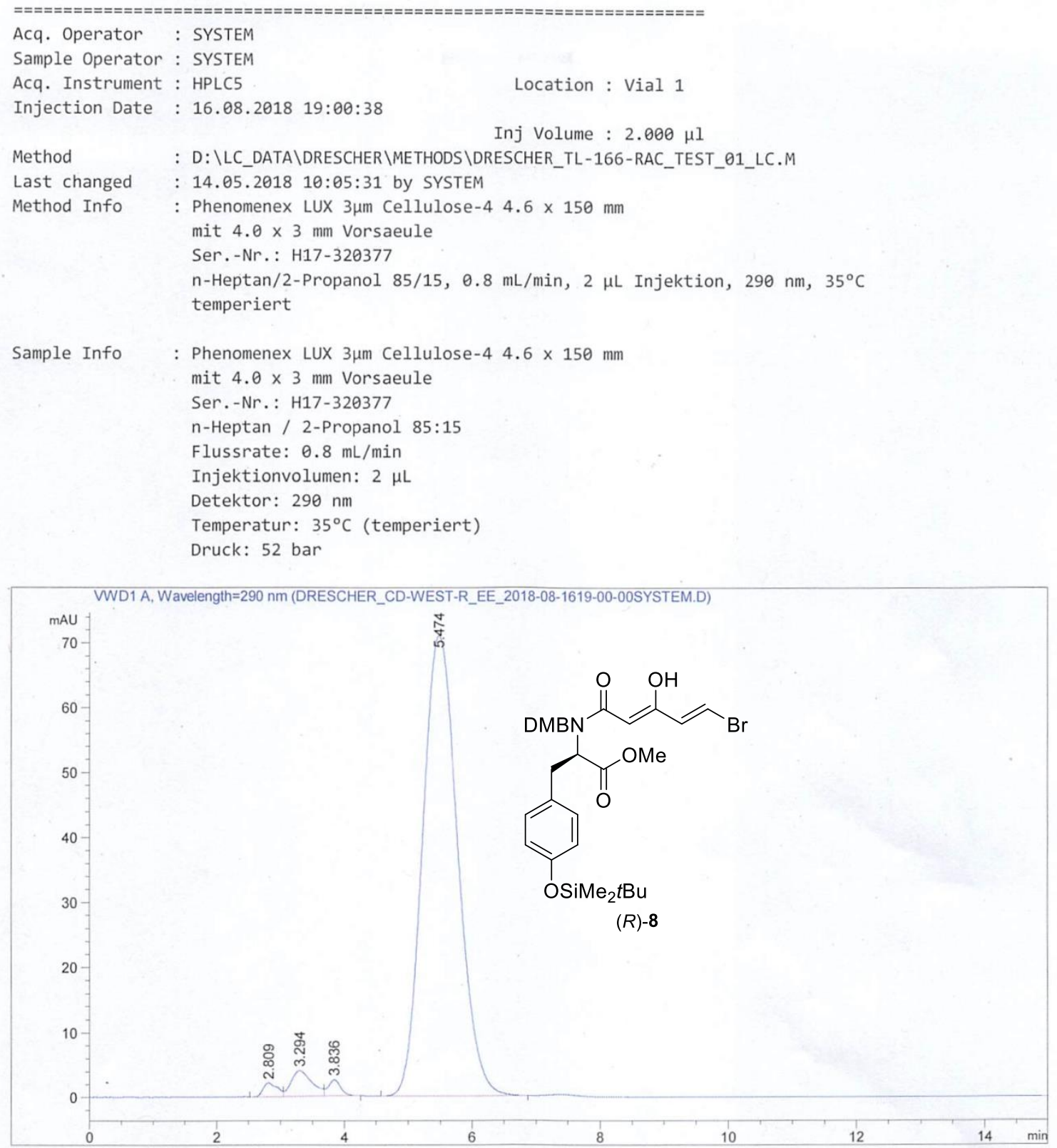
Christian Drescher, Morris Keller, Olivier Potterat, Matthias Hamburger and Reinhard Brückner, Structure-Elucidating Total Synthesis of the (Polyenoyl)tetramic Acid Militarinone C

Data File D: \LC_DATA \DRESCHER \DATA \DRESCHER_CD-WEST-R_EE_2018-08-1619-00-00SYSTEM.D Sample Name: Drescher_CD-West-R_ee_

Signal 1: VWD1 A, Wavelength $=290 \mathrm{~nm}$

\begin{tabular}{|c|c|c|c|c|c|c|}
\hline $\begin{array}{c}\text { Peak } \\
\#\end{array}$ & $\begin{array}{c}\text { RetTime } \\
\text { [min] }\end{array}$ & Type & $\begin{array}{l}\text { Width } \\
\text { [min] }\end{array}$ & $\begin{array}{c}\text { Area } \\
{\left[\mathrm{MAU}^{\star} \mathrm{s}\right]}\end{array}$ & $\begin{array}{l}\text { Height } \\
\text { [MAU] }\end{array}$ & $\begin{array}{c}\text { Area } \\
8\end{array}$ \\
\hline & & & & & & \\
\hline 1 & 2.809 & BV & 0.2286 & 34.12468 & 2.17030 & 1.1787 \\
\hline 2 & 3.2 & VV & 0.3314 & 90.09335 & 3.97439 & 3.1120 \\
\hline 3 & 3.836 & VB & 0.1991 & 34.04159 & 2.55474 & 1.1759 \\
\hline 4 & 5.474 & BB & 0.5958 & 2727.04272 & 70.90721 & 94.1965 \\
\hline 5 & 7.431 & MM & 0.5687 & 9.75443 & $2.85860 \mathrm{e}-1$ & 0.3369 \\
\hline & & & & & 89250 & \\
\hline
\end{tabular}

*** End of Report *** 
Christian Drescher, Morris Keller, Olivier Potterat, Matthias Hamburger and Reinhard Brückner, Structure-Elucidating Total Synthesis of the (Polyenoyl)tetramic Acid Militarinone C

\section{3. $\quad{ }^{1} \mathrm{H}$ and ${ }^{13} \mathrm{C}$ NMR Spectra in Numerical Order}

$3.1{ }^{1} \mathrm{H}$ and ${ }^{13} \mathrm{C}$ NMR Spectra of the Western Block 8 


\section{trans-3-Bromacrylic acid (14)}

${ }^{1} \mathbf{H}$ NMR (300.13 MHz, $\left.\mathrm{CDCl}_{3}\right)$ :

HO $\frac{\mathrm{O}}{1} / \mathrm{C}_{3}$

14

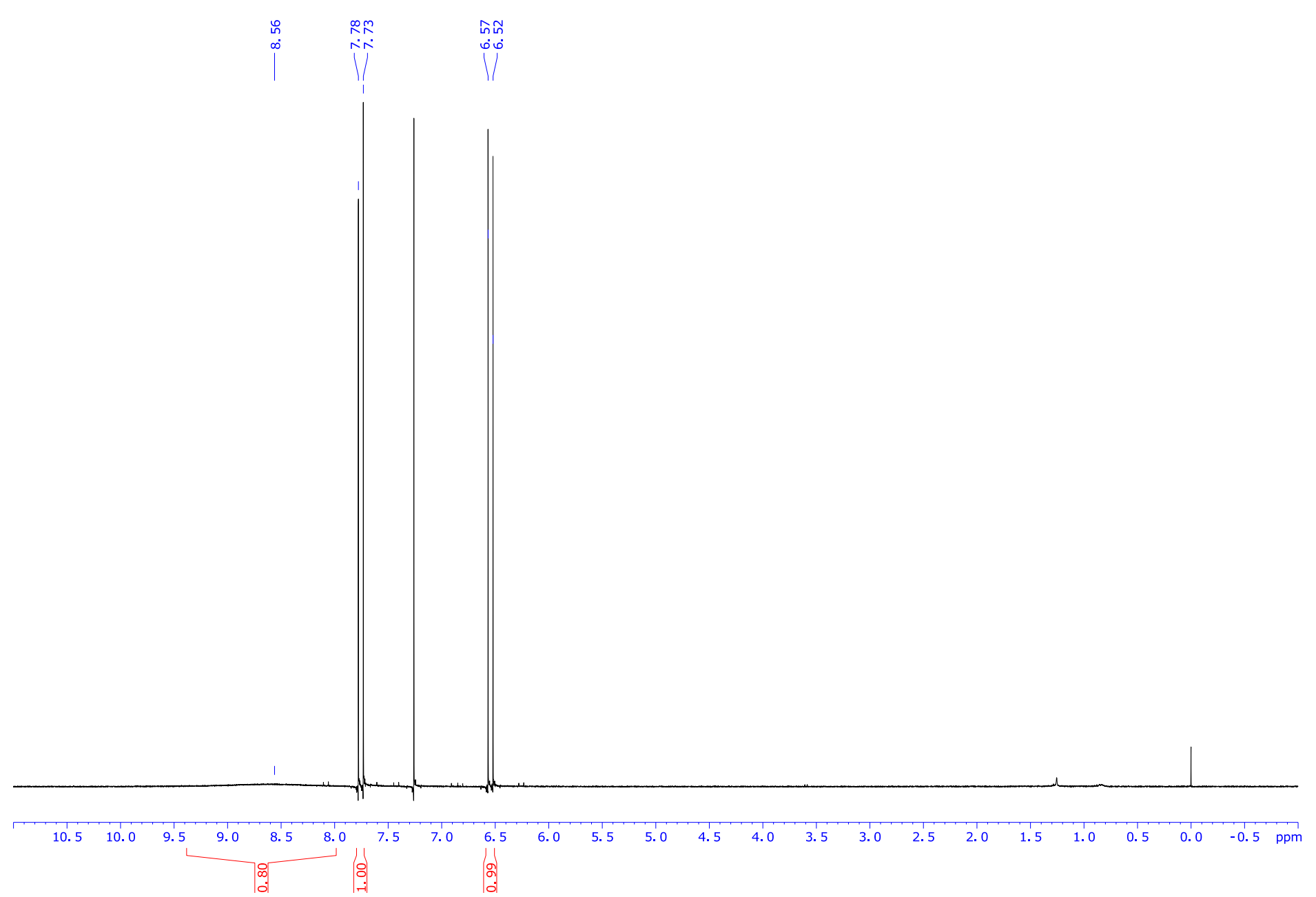




\section{$N$-Methylmethoxyamine (SI-1)}

${ }^{1} \mathbf{H}$ NMR (300.13 MHz, $\left.\mathrm{CDCl}_{3}\right)$ :<smiles>CNOC</smiles>

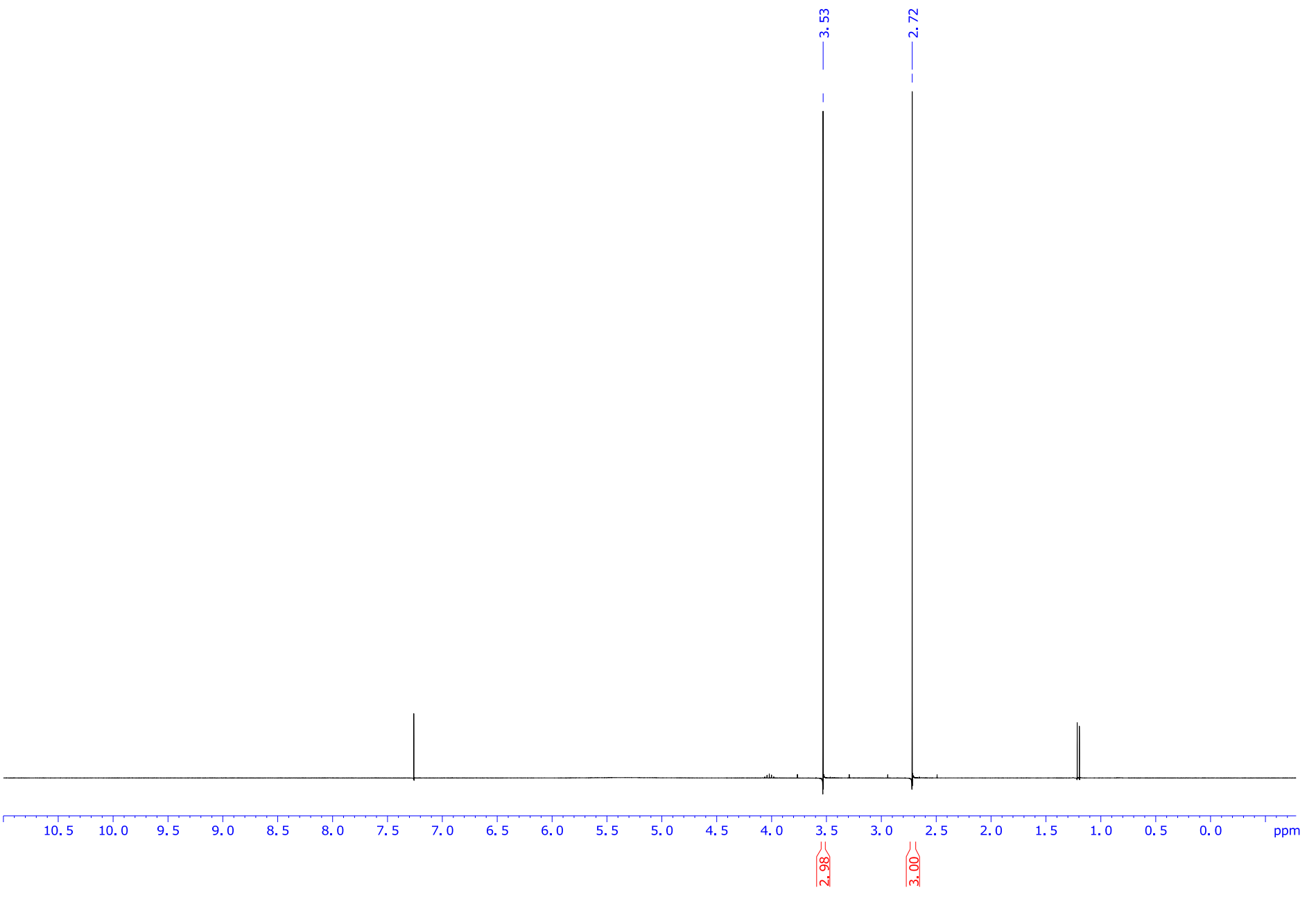


trans-3-Bromo- $N$-methoxy- $N$-methylacrylamide (23)

${ }^{1} \mathbf{H}$ NMR (300.13 MHz, $\left.\mathrm{CDCl}_{3}\right)$ :

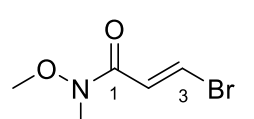

23

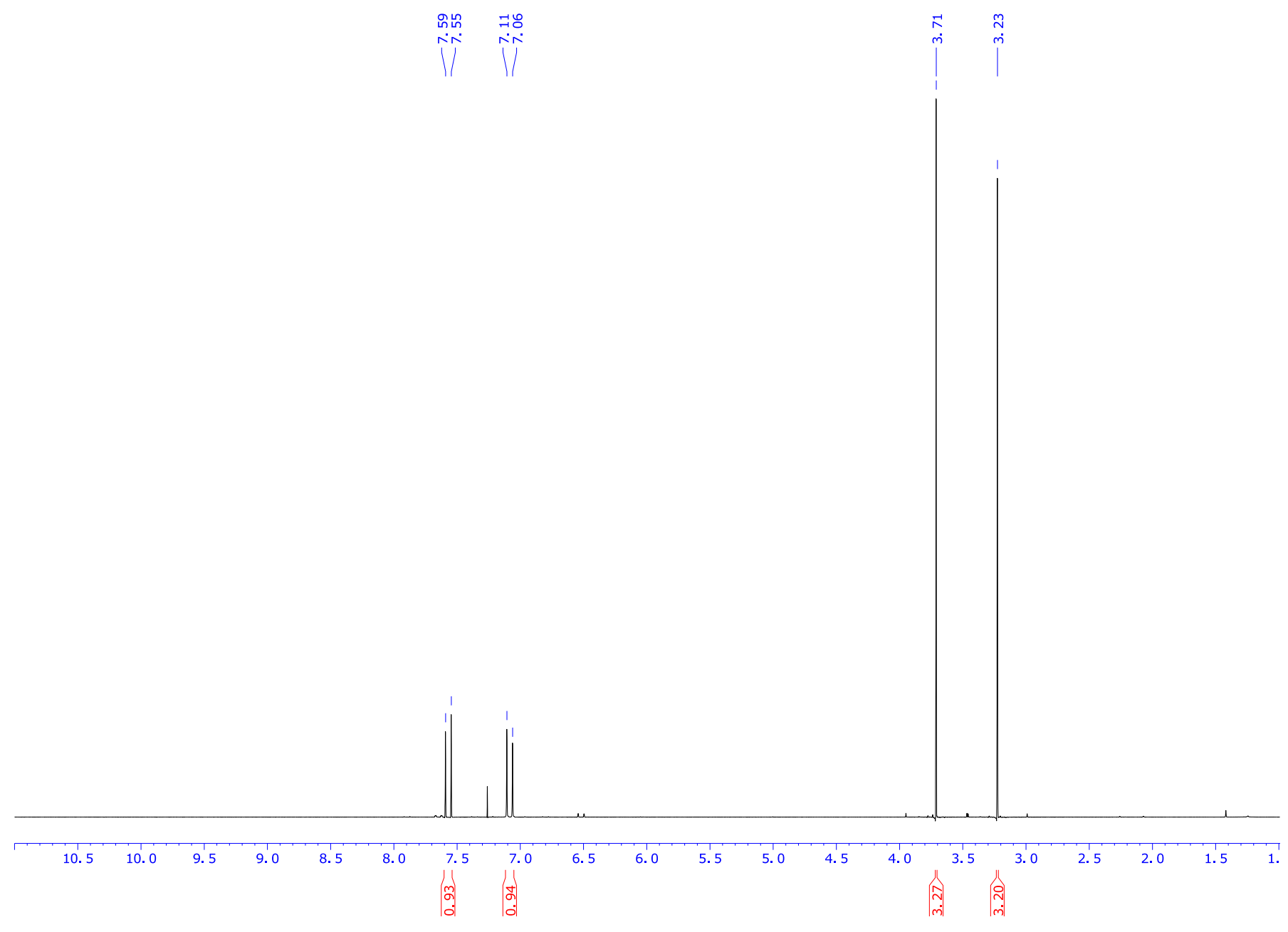




\section{$t$-Butylthioacetate (24)}

${ }^{1} \mathbf{H}$ NMR $\left(300.13 \mathrm{MHz}, \mathrm{CDCl}_{3}\right)$ :<smiles>CC(=O)SC(C)(C)C</smiles>

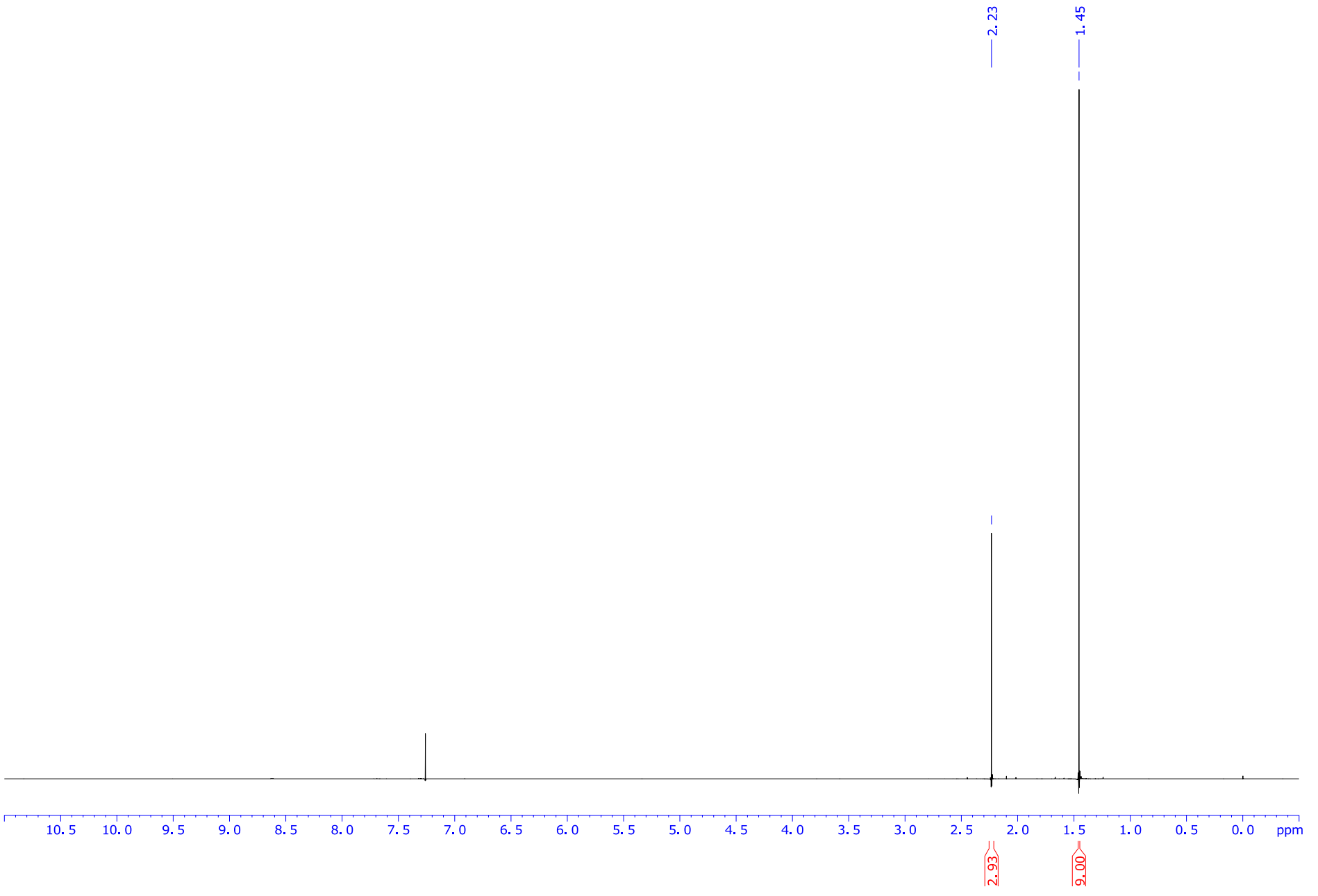




\section{S-tert-Butyl (E,E)-5-Bromo-3-hydroxypenta-2,4-dienethioate [enol-(11)]}

${ }^{1} \mathbf{H}$ NMR (300.13 MHz, $\left.\mathrm{CDCl}_{3}\right)$ :
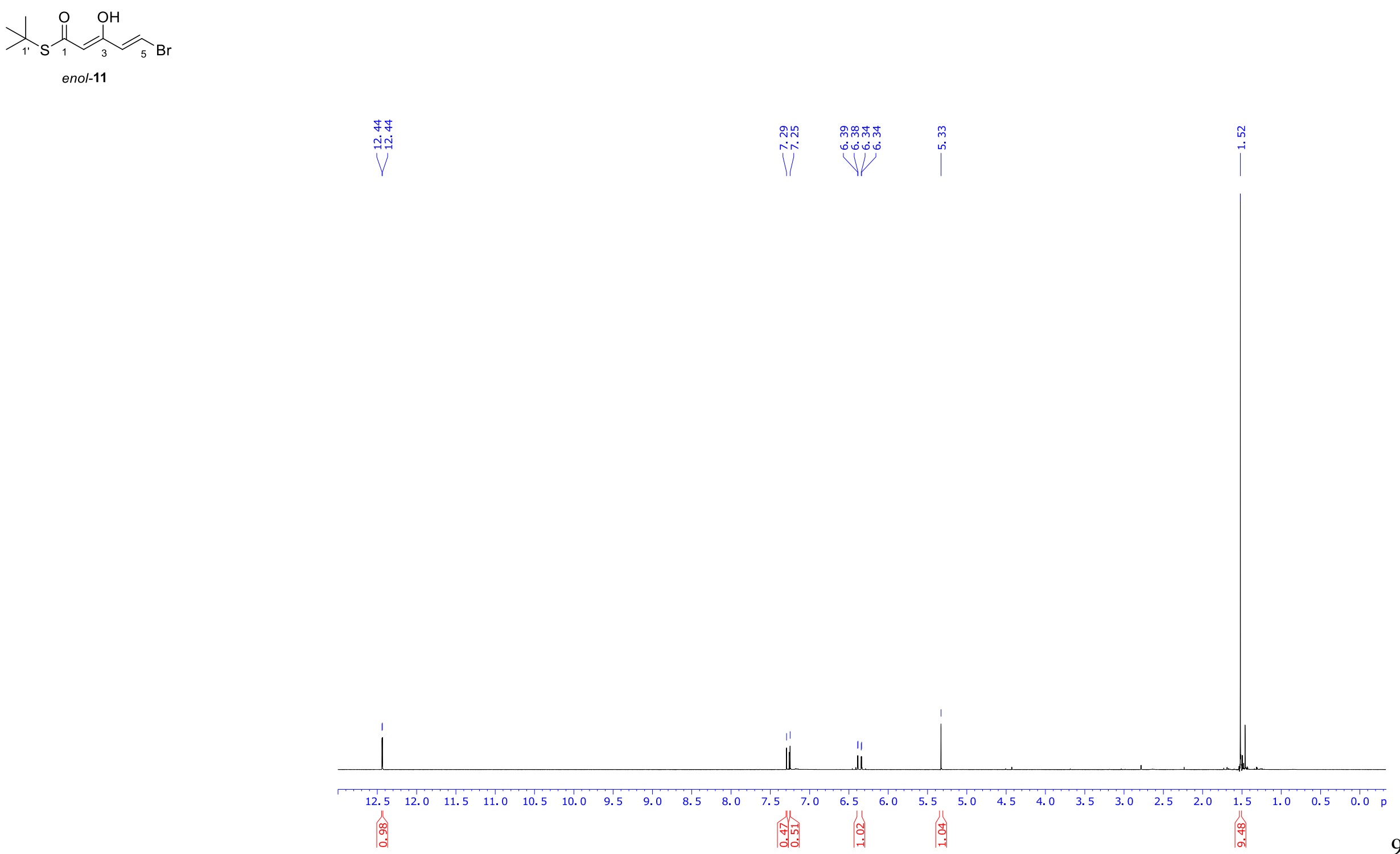


\section{Methyl (2S)-Amino-3-(4'-hydroxyphenyl)propanoate [(S)-20]}

and

\section{Methyl (2R)-Amino-3-(4'-hydroxyphenyl)propanoate [(R)-20]}

${ }^{1} \mathbf{H}$ NMR (300.13 MHz, $\left.\mathrm{D}_{2} \mathrm{O}\right)$ :

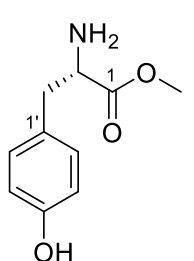

$(S)-20$

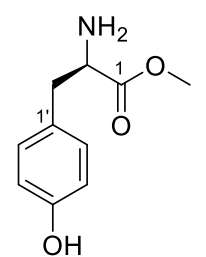

(R)-20

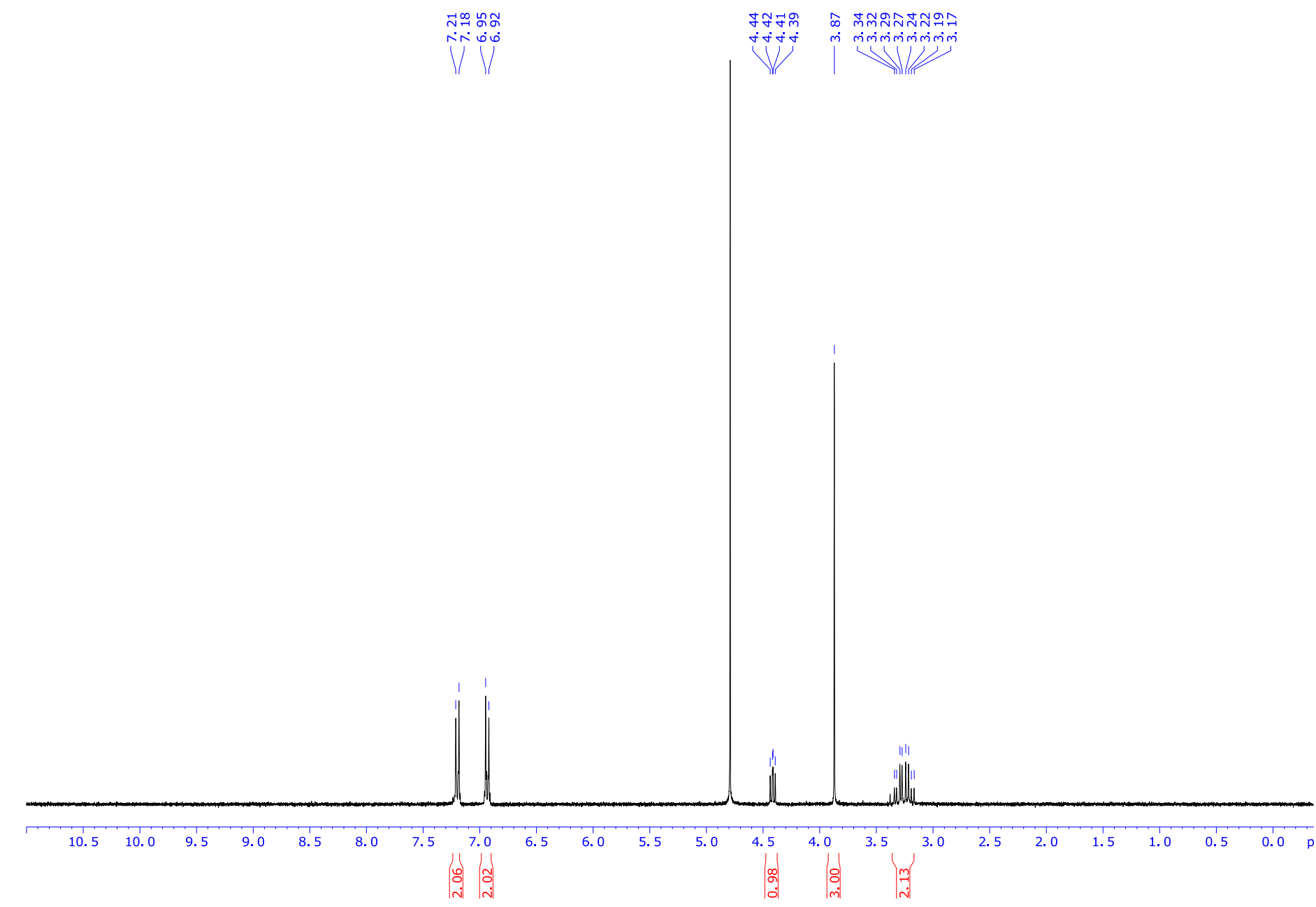


Methyl (2S)-2-Amino-3-\{4'-[(tert-butyldimethylsilyl)oxy]phenyl $\}$ propanoate $[(S)-21]$

and

Methyl (2R)-2-Amino-3-\{4'-[(tert-butyldimethylsilyl)oxy]phenyl $\}$ propanoate $[(R)-21]$

${ }^{1} \mathbf{H}$ NMR (300.13 MHz, $\left.\mathrm{CDCl}_{3}\right)$ :

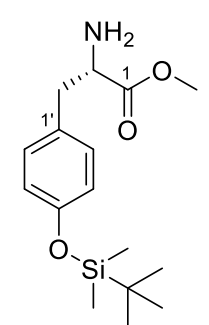

$(S)-21$

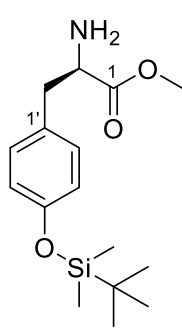

$(R)-21$

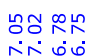

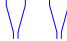

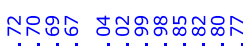
mining

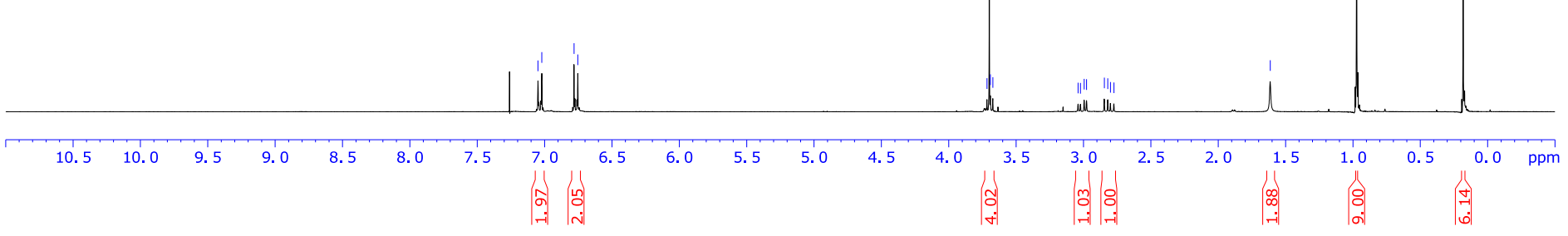


Methyl (2S)-3-\{4'-[(tert-Butyldimethylsilyl)oxy]phenyl\}-2-[(1",5"-dimethoxybenzyl)amino]propanoate $[(S)-13]$

and

Methyl (2R)-3-\{4'-[(tert-Butyldimethylsilyl)oxy]phenyl\}-2-[(1",5"'-dimethoxybenzyl)amino]propanoate [(R)-13]

${ }^{1} \mathbf{H}$ NMR (500.10 MHz, $\left.\mathrm{CDCl}_{3}\right)$ :

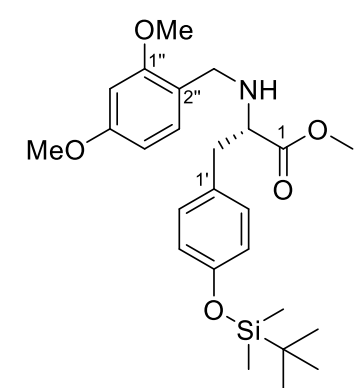

$(S)-13$

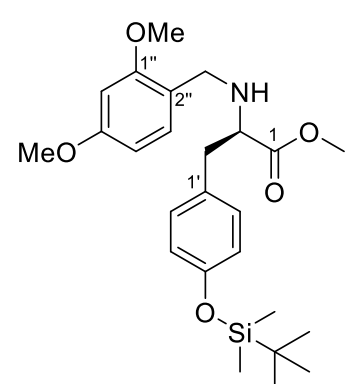

$(R)-13$

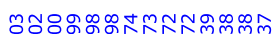

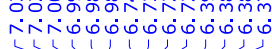

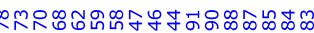
minimiminiminiminivivivin

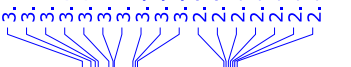


Methyl (2S)-3-\{4'-[(tert-Butyldimethylsilyl)oxy]phenyl\}-2-[(1",5"-dimethoxybenzyl)amino]propanoate $[(S)-13]$ and

Methyl (2R)-3-\{4'-[(tert-Butyldimethylsilyl)oxy]phenyl\}-2-[(1",5"'-dimethoxybenzyl)amino]propanoate [(R)-13] ${ }^{13}$ C NMR (125.75 MHz, $\left.\mathrm{CDCl}_{3}\right)$ :

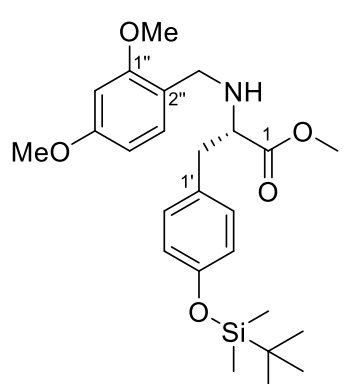

$(S)-13$

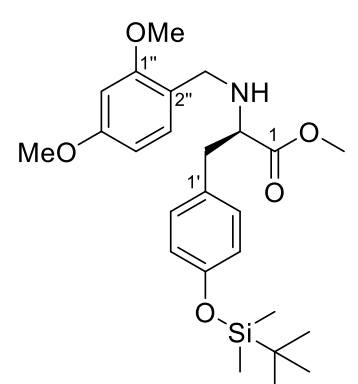

$(R)-13$

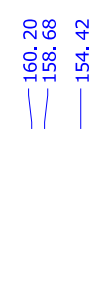

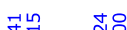

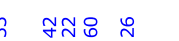

$\therefore \quad \stackrel{1}{0}$

$\forall|+| \stackrel{\infty}{\stackrel{\infty}{\infty}} \mid$

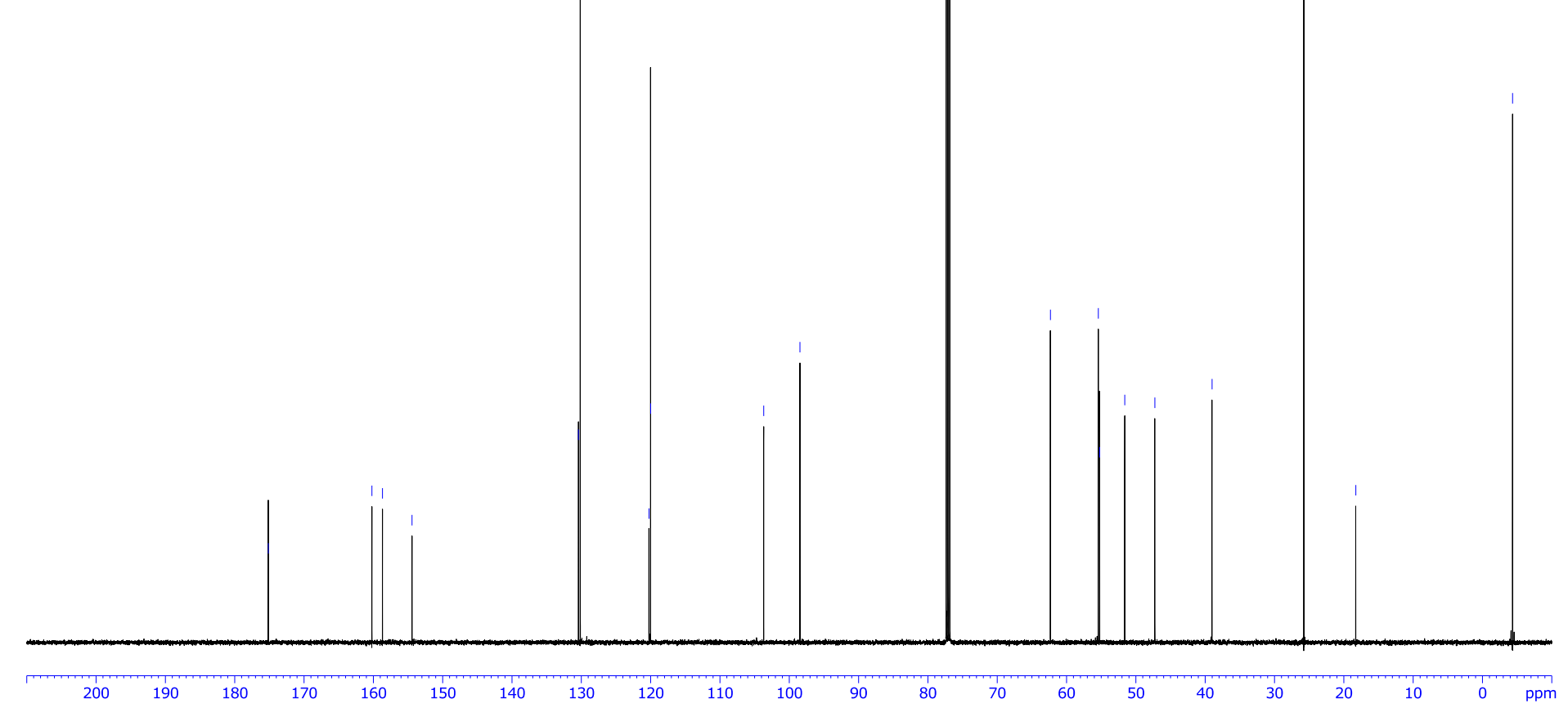


Methyl (2'S)-2'-\{N-[(1E,2Z,4E)-5-Bromo-3-hydroxy-1-oxoypenta-2,4-dienyl]- $N$-(1'"',5'"-dimethoxybenzyl) $\}-3$ '- $\{1$ "'-[(tertbutyldimethylsilyl)oxy]phenyl $\}$ propanoate $[$ E-enol-(S)-8]

and

Methyl (2'R)-2'-\{N-[(1E,2Z,4E)-5-Bromo-3-hydroxy-1-oxoypenta-2,4-dienyl]- $N$-(1'",5"'-dimethoxybenzyl) $\}-3$ '- $\left\{1^{\prime \prime}-[(t e r t-\right.$ butyldimethylsilyl)oxy]phenyl $\}$ propanoate $[$ E-enol-(R)-8]

${ }^{1} \mathbf{H}$ NMR $\left(500.10 \mathrm{MHz}, \mathrm{CDCl}_{3}\right)$ :
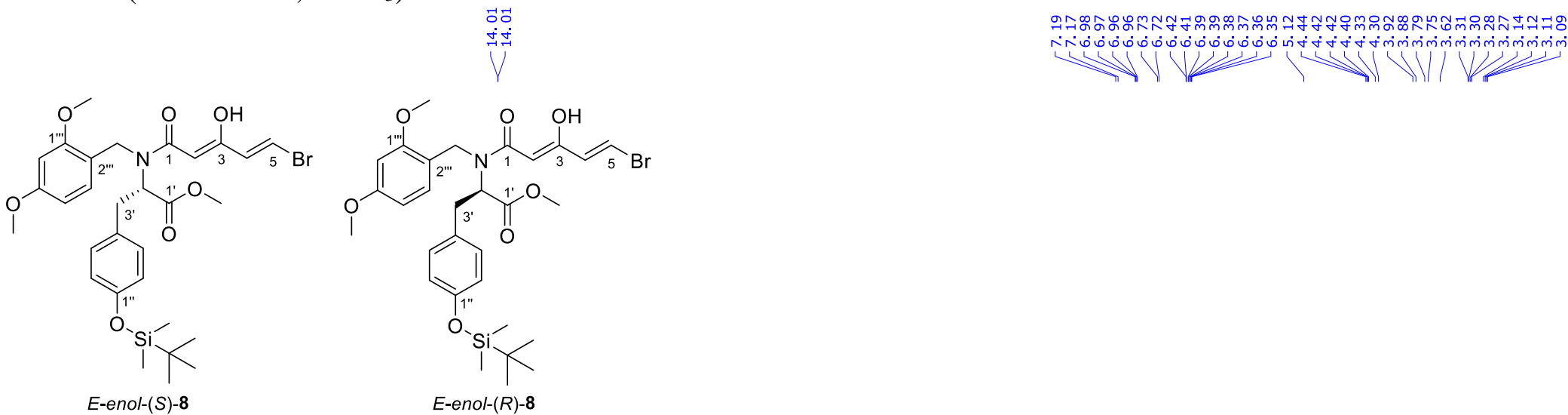

E-enol-(S)-8

E-enol-(R)-8

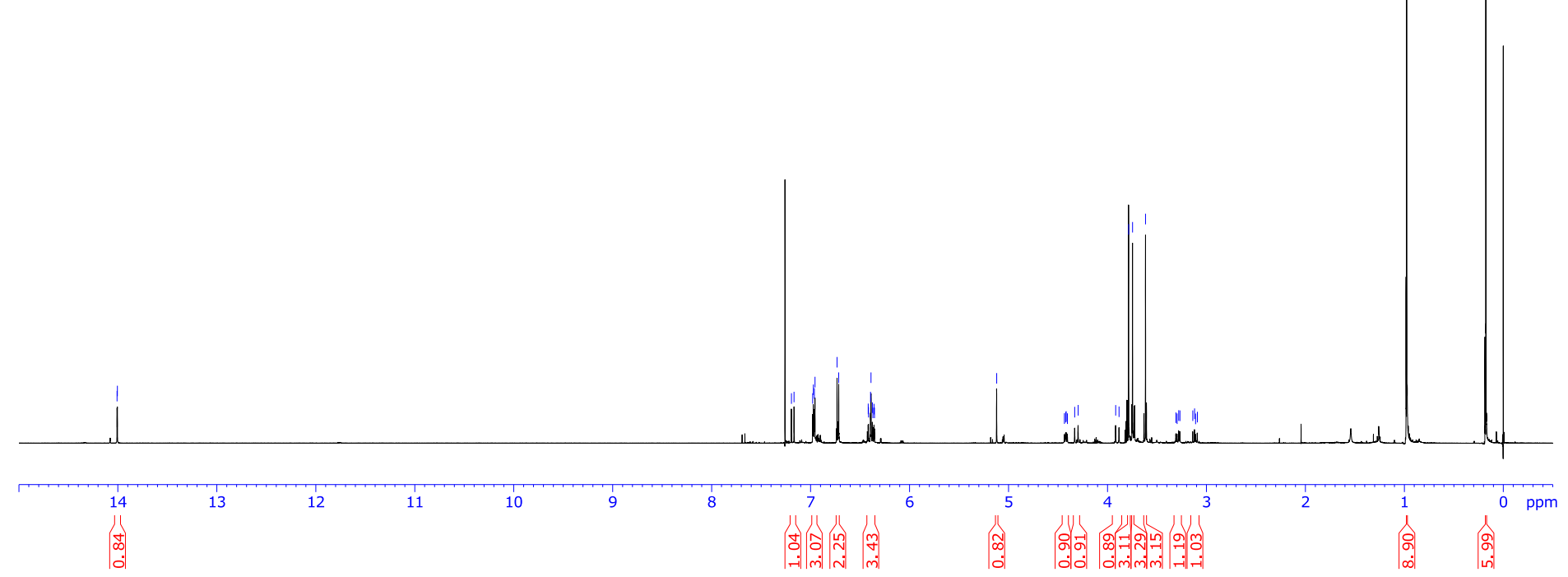


Methyl (2'S)-2'-\{N-[(1E,2Z,4E)-5-Bromo-3-hydroxy-1-oxoypenta-2,4-dienyl]-N-(1"',5'"-dimethoxybenzyl) $\}-3$ '- $\{1 "$ "-[(tertbutyldimethylsilyl)oxy]phenyl $\}$ propanoate $[$ E-enol-(S)-8]

$$
\text { and }
$$

Methyl (2'R)-2'-\{N-[(1E,2Z,4E)-5-Bromo-3-hydroxy-1-oxoypenta-2,4-dienyl]- $N$-(1'"',5"'-dimethoxybenzyl) $\}-3$ '- $\{1$ '"-[(tertbutyldimethylsilyl)oxy]phenyl $\}$ propanoate $[E-e n o l-(R)-8]$

${ }^{13}$ C NMR (125.75 MHz, $\left.\mathrm{CDCl}_{3}\right)$ :
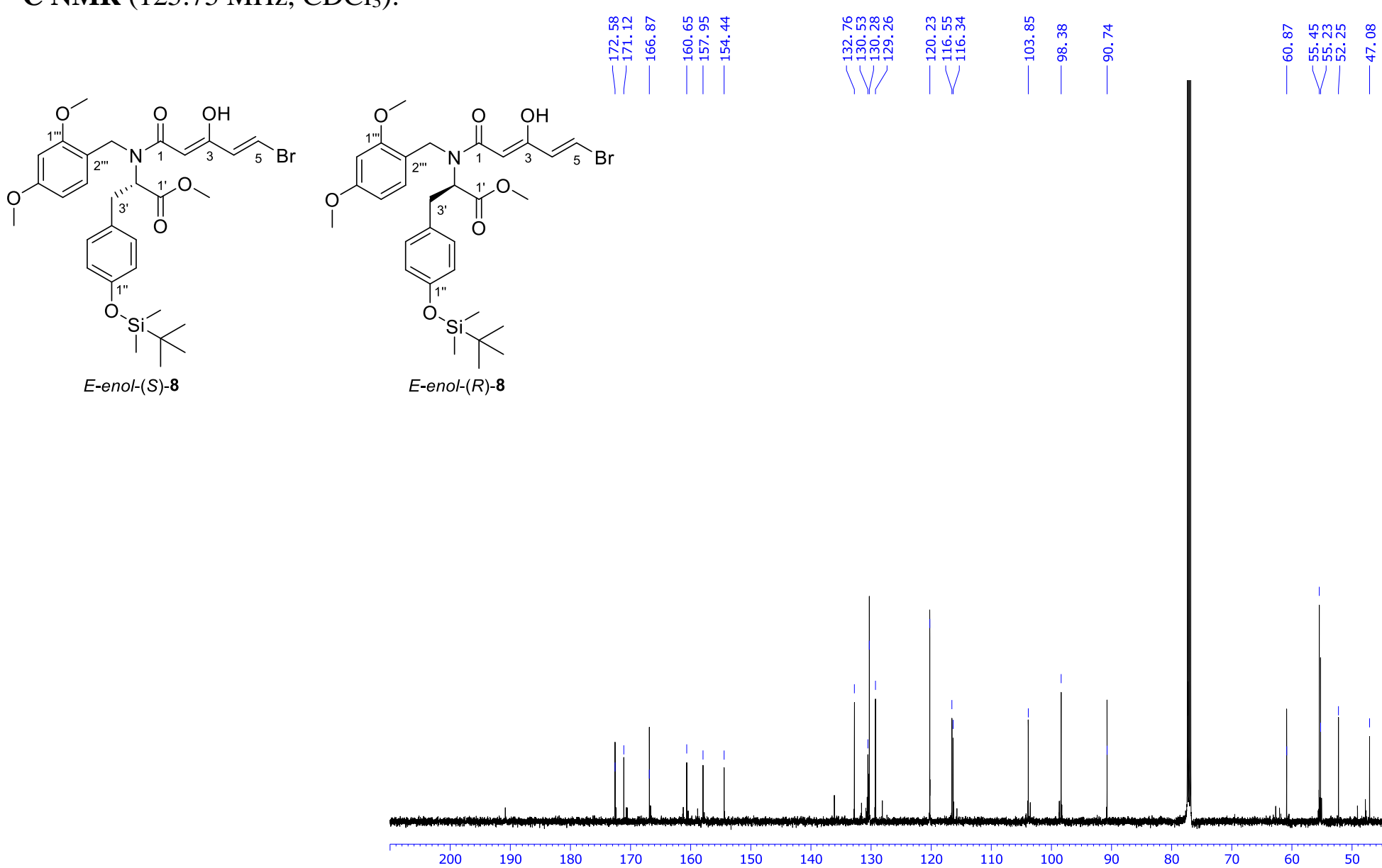
Christian Drescher, Morris Keller, Olivier Potterat, Matthias Hamburger and Reinhard Brückner, Structure-Elucidating Total Synthesis of the (Polyenoyl)tetramic Acid Militarinone C

\subsection{NMR Spectra of the Eastern Block Precursor 32}


$N$-[(1Z,1'S,2'S)-2'-Hydroxy-2'-phenylpropan-1'-yl]- $N$-methylpropionamide $[(Z, S, S)-18]$

and

$N$-[(1Z,1'R,2'R)-2'-Hydroxy-2'-phenylpropan-1'-yl]- $N$-methylpropionamide $[(Z, R, R)-18]$

${ }^{1} \mathbf{H}$ NMR $\left(300.13 \mathrm{MHz}, \mathrm{C}_{6} \mathrm{D}_{6}\right)$ :

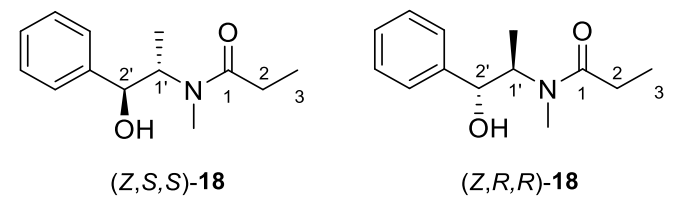




\section{$(1 Z, 2 R)-N-\left[\left(1^{\prime} S, 2 ' S\right)-2\right.$ '-Hydroxy-2'-phenylpropan-1'-yl]-N,2-dimethylbutanamide $(Z, S, S, R)$-26}

${ }^{1} \mathbf{H}$ NMR (300.13 MHz, $\left.\mathrm{CDCl}_{3}\right)$ :

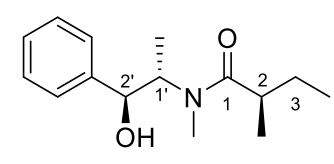

$(Z, S, S, R)-26$

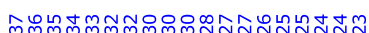

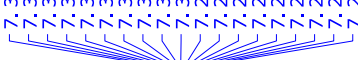

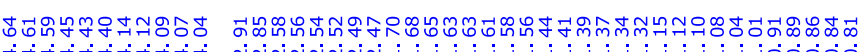

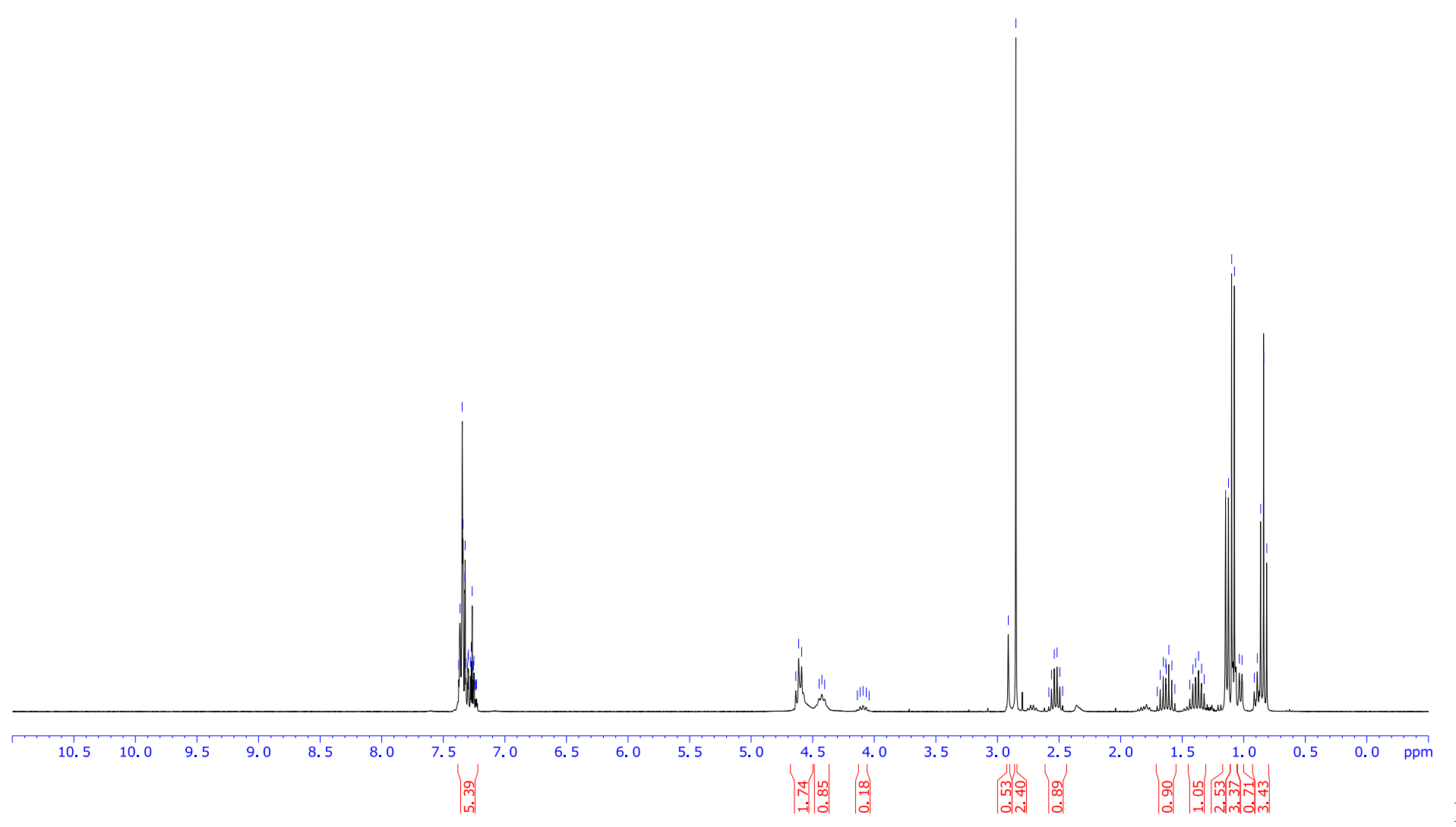




\section{(R)-2-Methylbutanol [(R)-19]}

${ }^{1} \mathbf{H}$ NMR (300.13 MHz, $\left.\mathrm{CDCl}_{3}\right)$ :

$\mathrm{OH}$

$$
\text { 13 }
$$

$(R)-19$ 
(R)-2-Methylbutyl 4-Methylbenzenesulfonate [(R)-27]

and

(S)-2-Methylbutyl 4-Methylbenzenesulfonate [(S)-27]

${ }^{1} \mathbf{H}$ NMR (300.13 MHz, $\left.\mathrm{CDCl}_{3}\right)$ :

$\left.\left.\right|_{(R)-27} ^{\text {OTs }}\right|_{\substack{\vdots \\(S)-27}} ^{\text {OTs }} \underbrace{2}{ }^{2}$

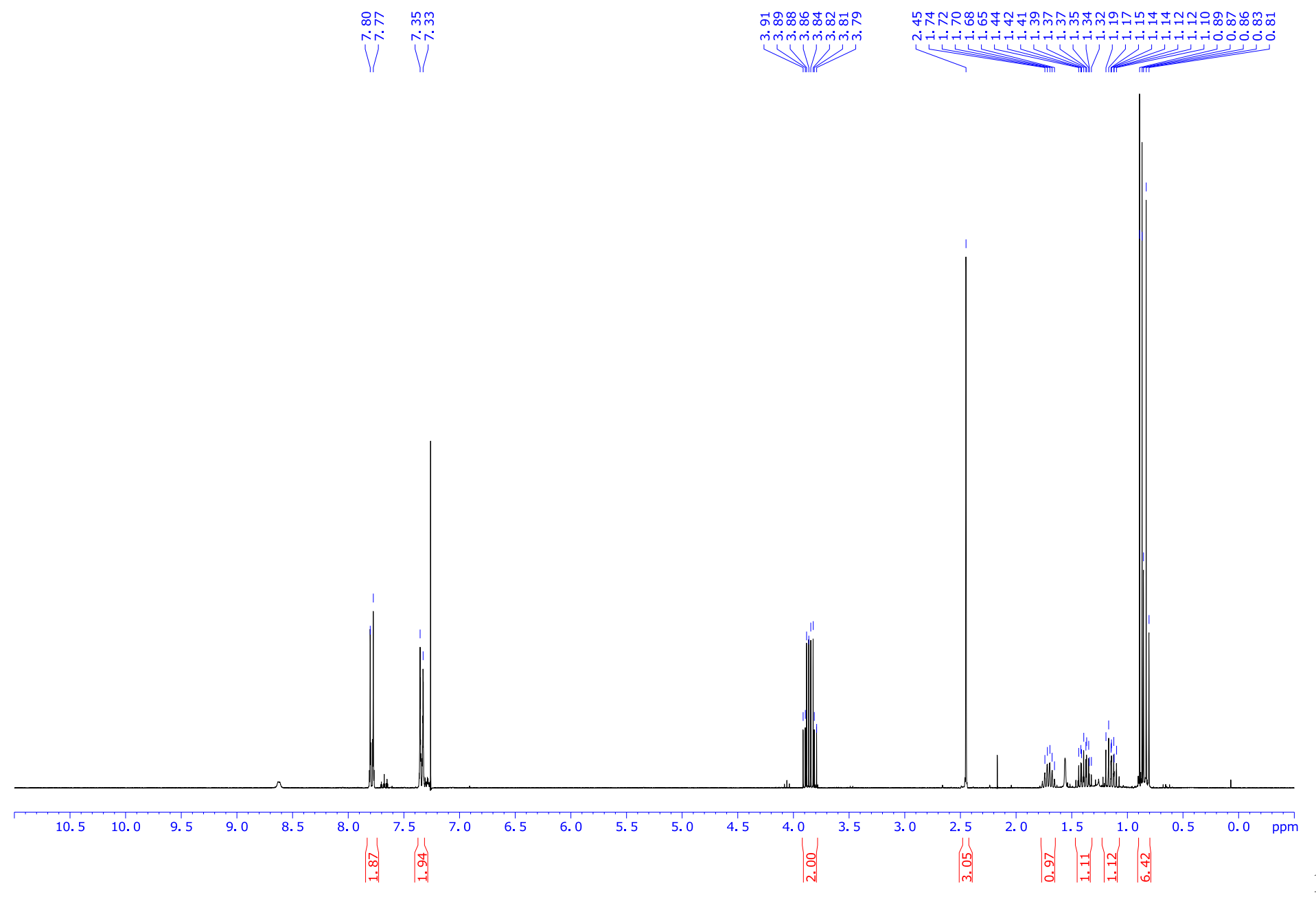




\section{(R)-1-Iodo-2-methylbutane [(R)-28]}

and

\section{(S)-1-Iodo-2-methylbutane [(S)-28]}

${ }^{\mathbf{1}} \mathbf{H}$ NMR (300.13 MHz, $\mathrm{CDCl}_{3}$ ):

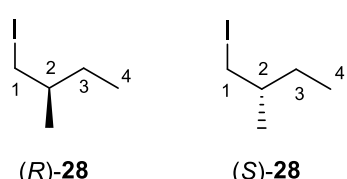


$(1 Z, 2 R, 4 R)-N$-[(1'S,2'S)-2'-Hydroxy-1'-methyl-2'-phenylethyl]- $N$-methyl-2,4-dimethylhexanamide [(Z,S,S,R,R)-15]

and

$(1 Z, 2 S, 4 S)-N$-[(1'R,2'R)-2'-Hydroxy-1'-methyl-2'-phenylethyl]- $N$-methyl-2,4-dimethylhexanamide [(Z,R,R,S,S)-15]

${ }^{1} \mathbf{H}$ NMR (300.13 MHz, $\left.\mathrm{CDCl}_{3}\right)$ :

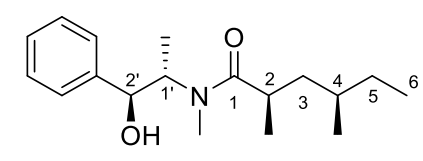

$(Z, S, S, R, R)-15$

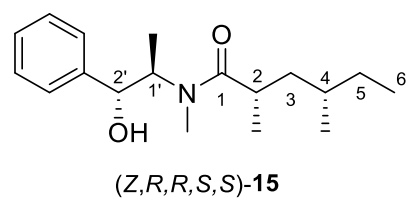

$(Z, R, R, S, S)-15$

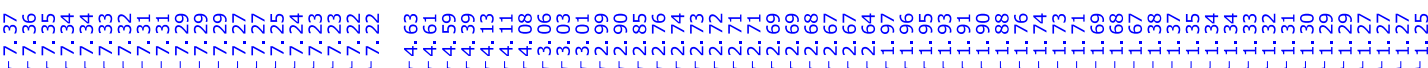

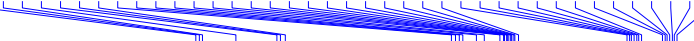

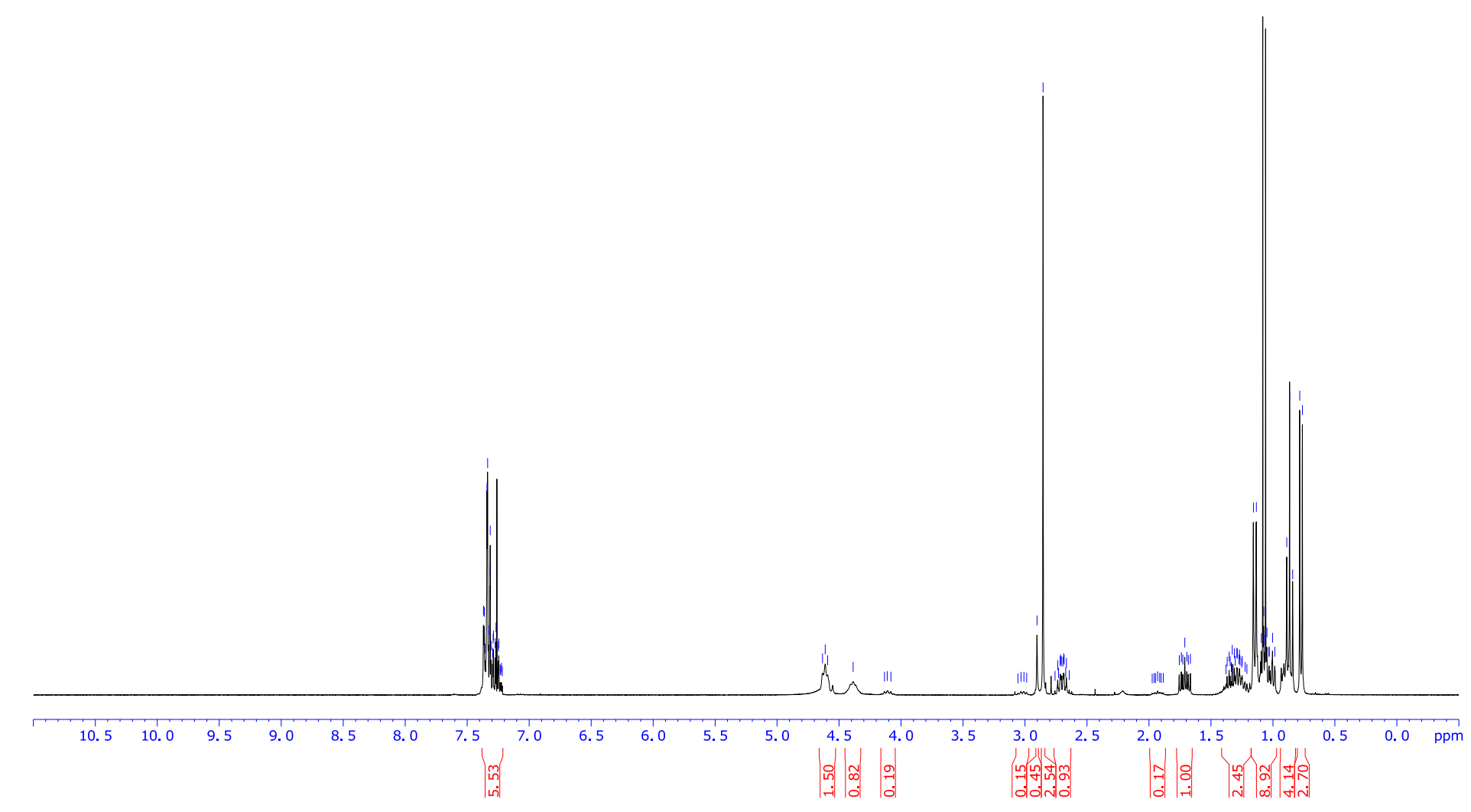




\section{$(2 R, 4 R)$-2,4-Dimethylhexan-1-ol $[(R, R)-29]$}

and

\section{$(2 S, 4 S)$-2,4-Dimethylhexan-1-ol $[(S, S)-29]$}

${ }^{\mathbf{1}} \mathbf{H}$ NMR $\left(500.10 \mathrm{MHz}, \mathrm{CDCl}_{3}\right)$ :

$\left.\right|_{(R, R)-29} ^{2}$

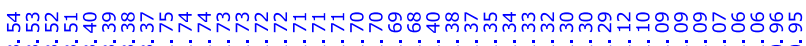

$(R, R)-29 \quad(S, S)-29$
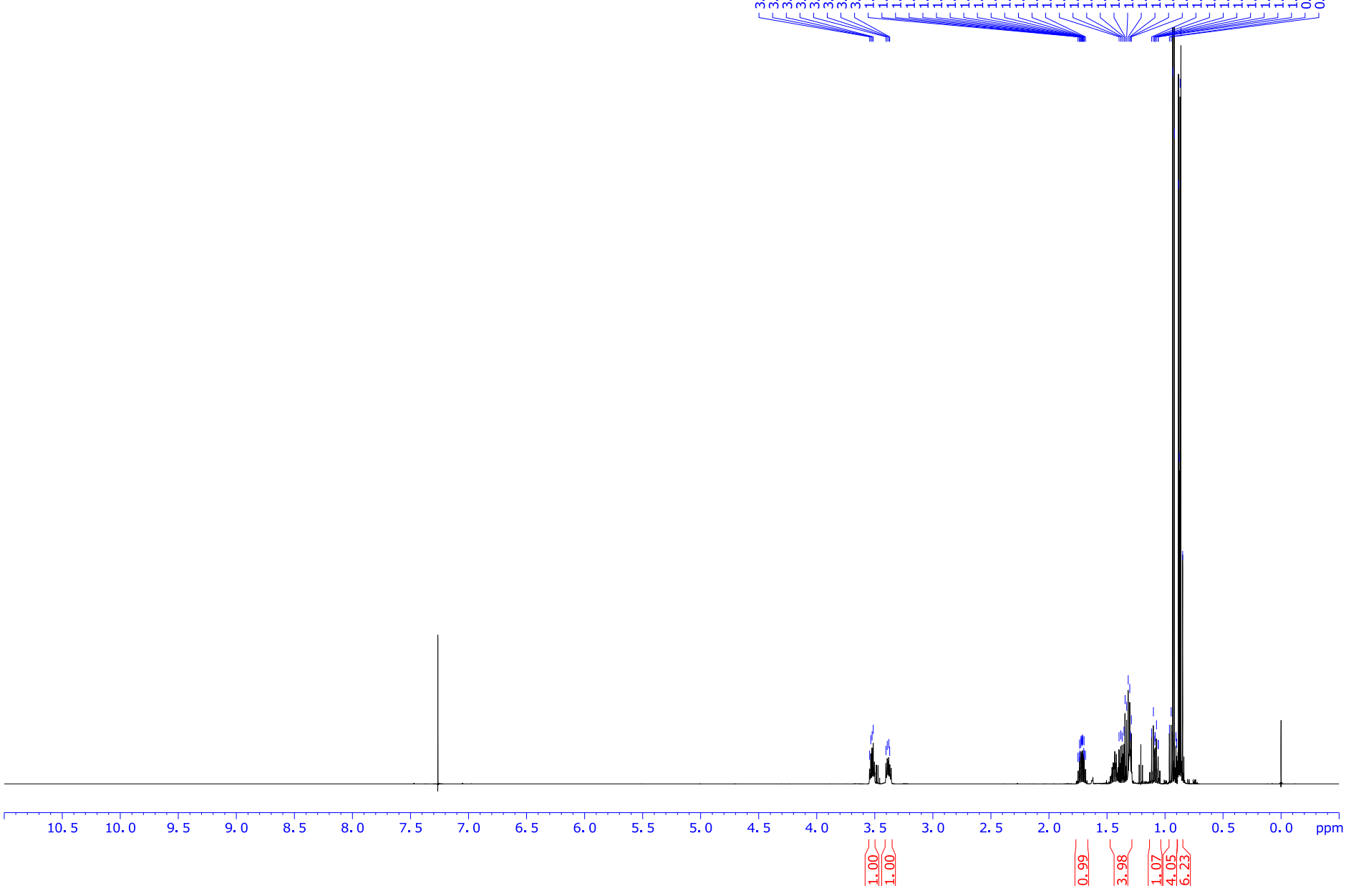


\section{$(2 R, 4 R)-2,4-D i m e t h y l h e x a n-1-o l ~[(R, R)-29]$}

and

\section{$(2 S, 4 S)$-2,4-Dimethylhexan-1-ol $[(S, S)-29]$}

${ }^{13}$ C NMR (125.75 MHz, $\left.\mathrm{CDCl}_{3}\right)$ :

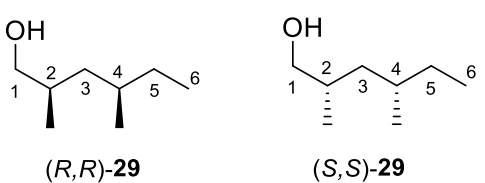

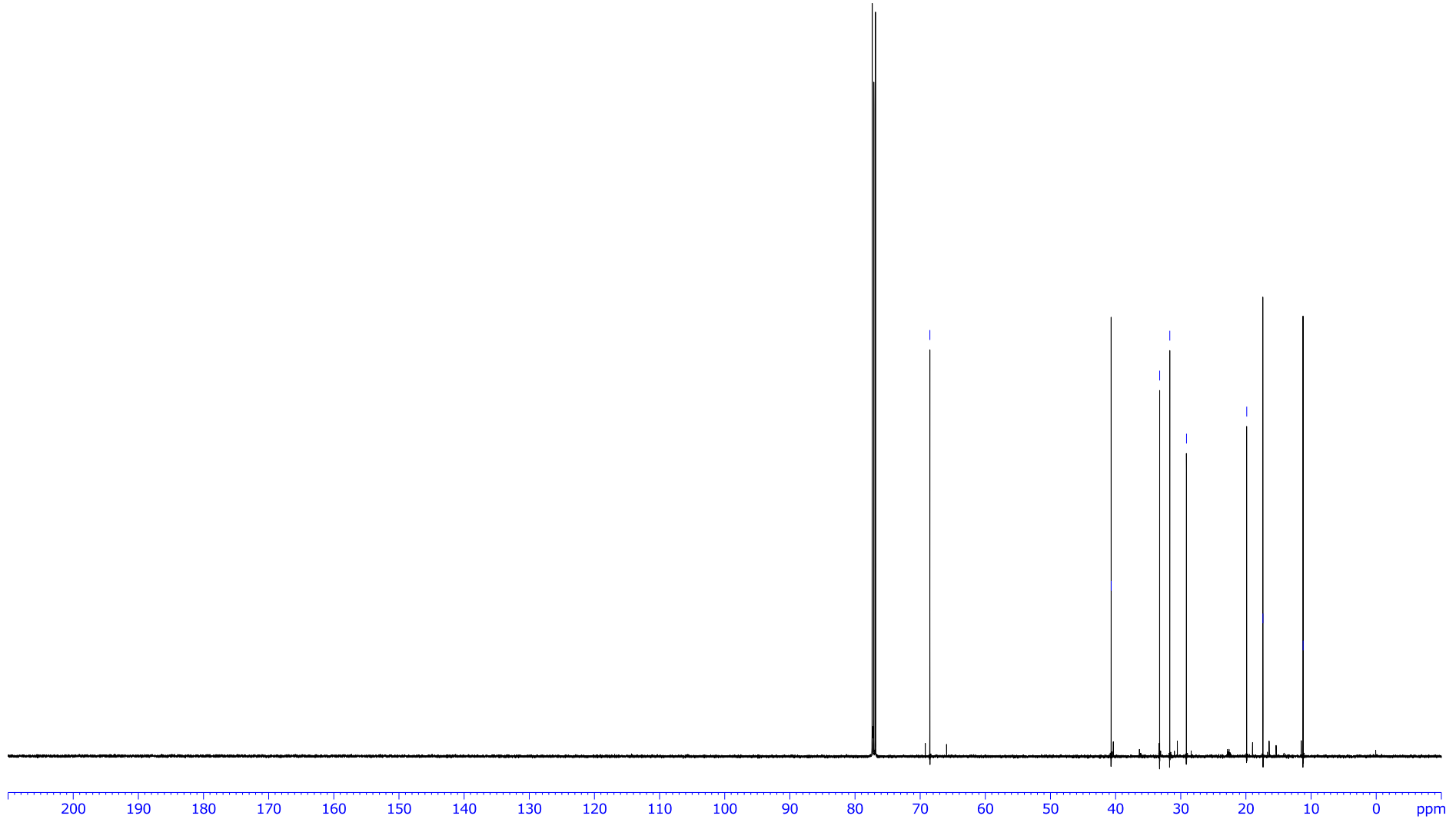




\section{$(2 R, 4 R)-2,4-D i m e t h y l h e x a n a l ~[(R, R)-12]$}

and

\section{$(2 S, 4 S)-2,4-D i m e t h y l h e x a n a l ~[(S, S)-12]$}

${ }^{1} \mathbf{H}$ NMR (300.13 MHz, $\mathrm{CDCl}_{3}$ ):

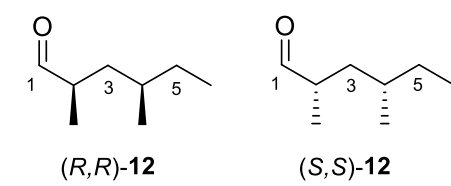

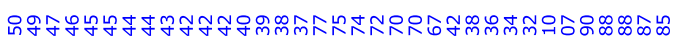
$(R, R)-12 \quad(S, S)-12$

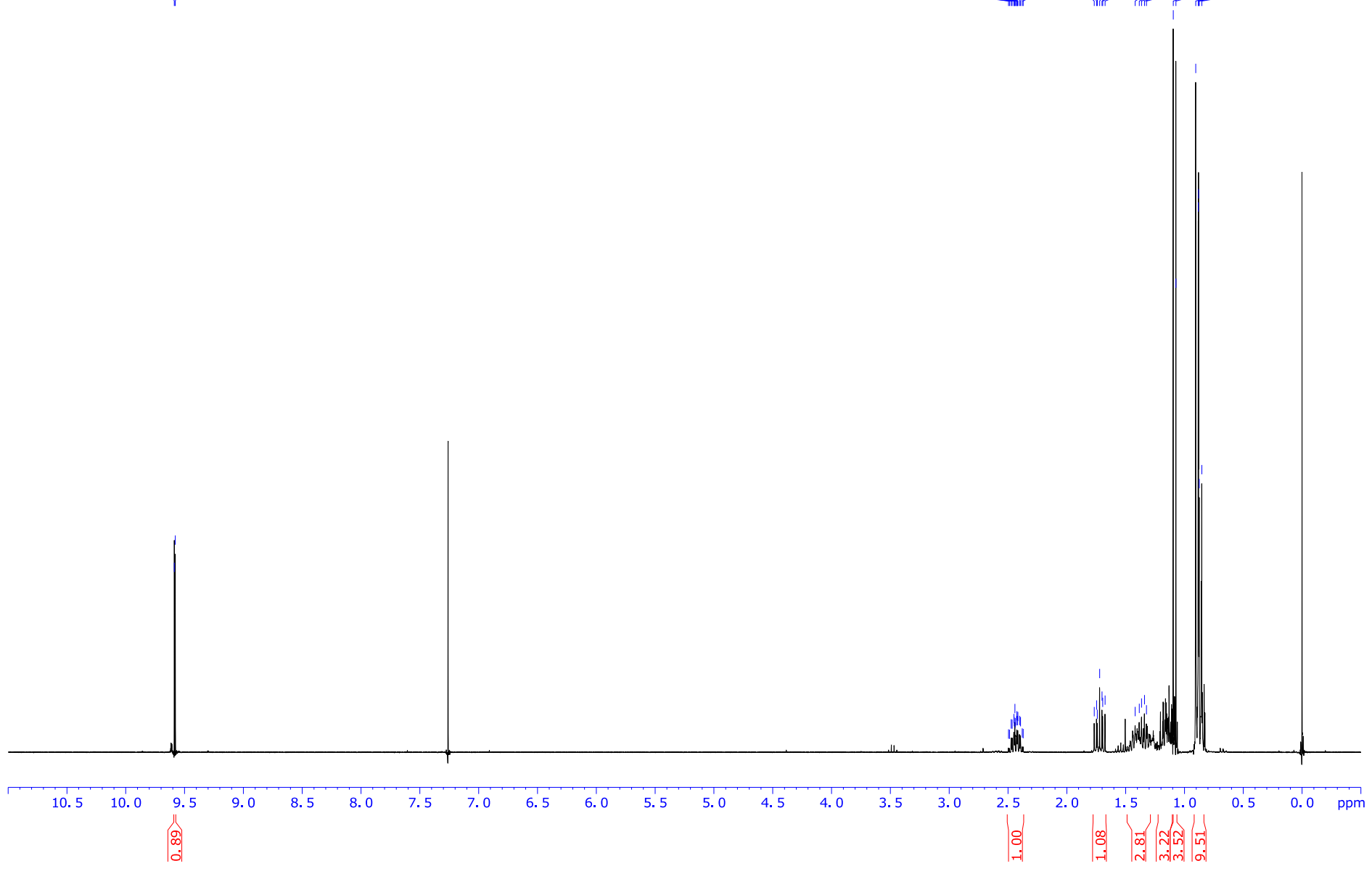




\section{(E)-N-Cyclohexylpropan-1-imine (SI-2)}

${ }^{1} \mathbf{H}$ NMR (300.13 MHz, $\mathrm{CDCl}_{3}$ ):

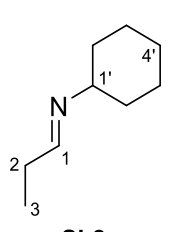

SI-2

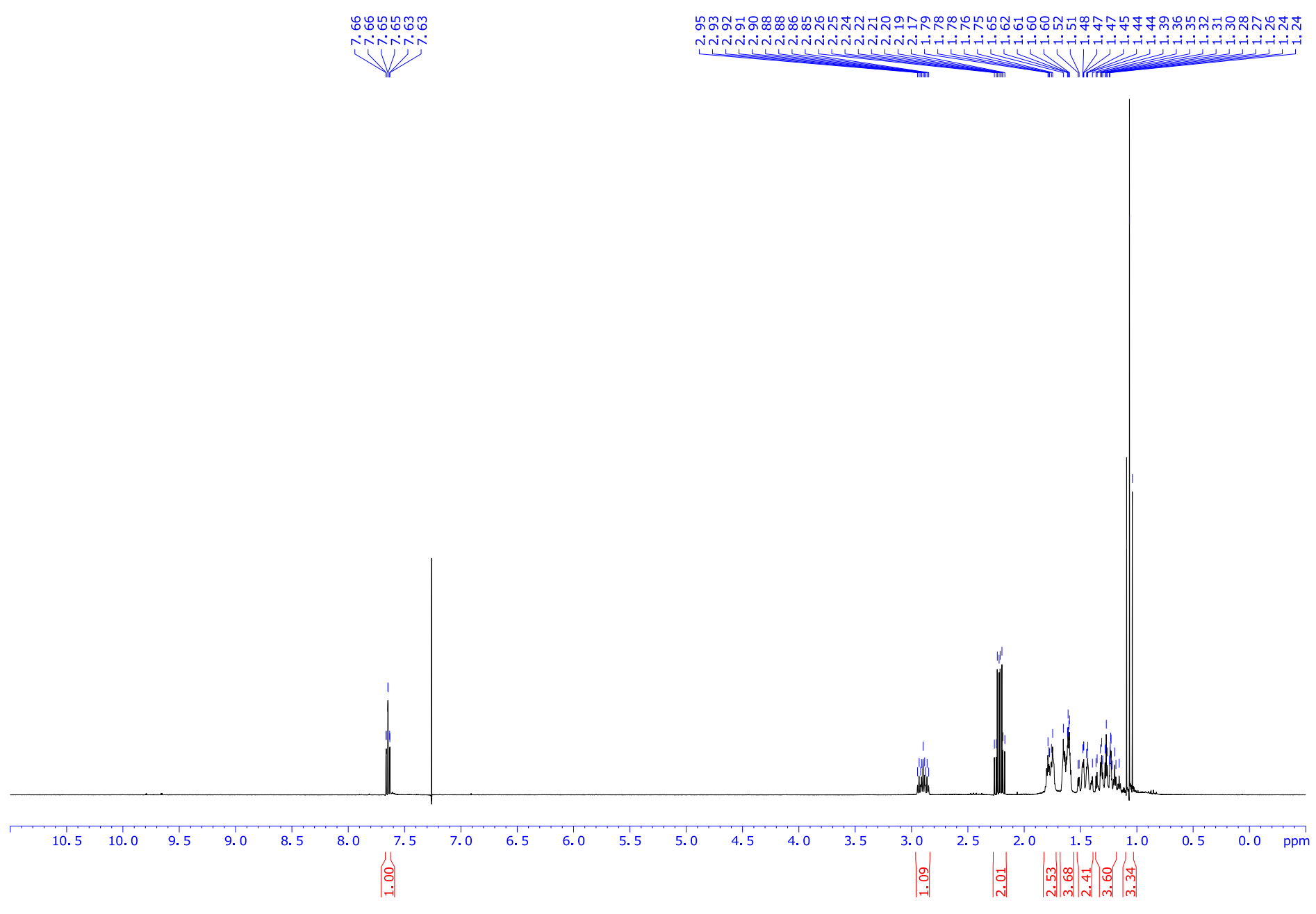




\section{(E)-N-Cyclohexyl-2-(triethylsilyl)propan-1-imine (30)}

${ }^{\mathbf{1}} \mathbf{H}$ NMR (300.13 MHz, $\mathrm{CDCl}_{3}$ ):

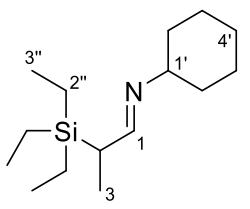

30

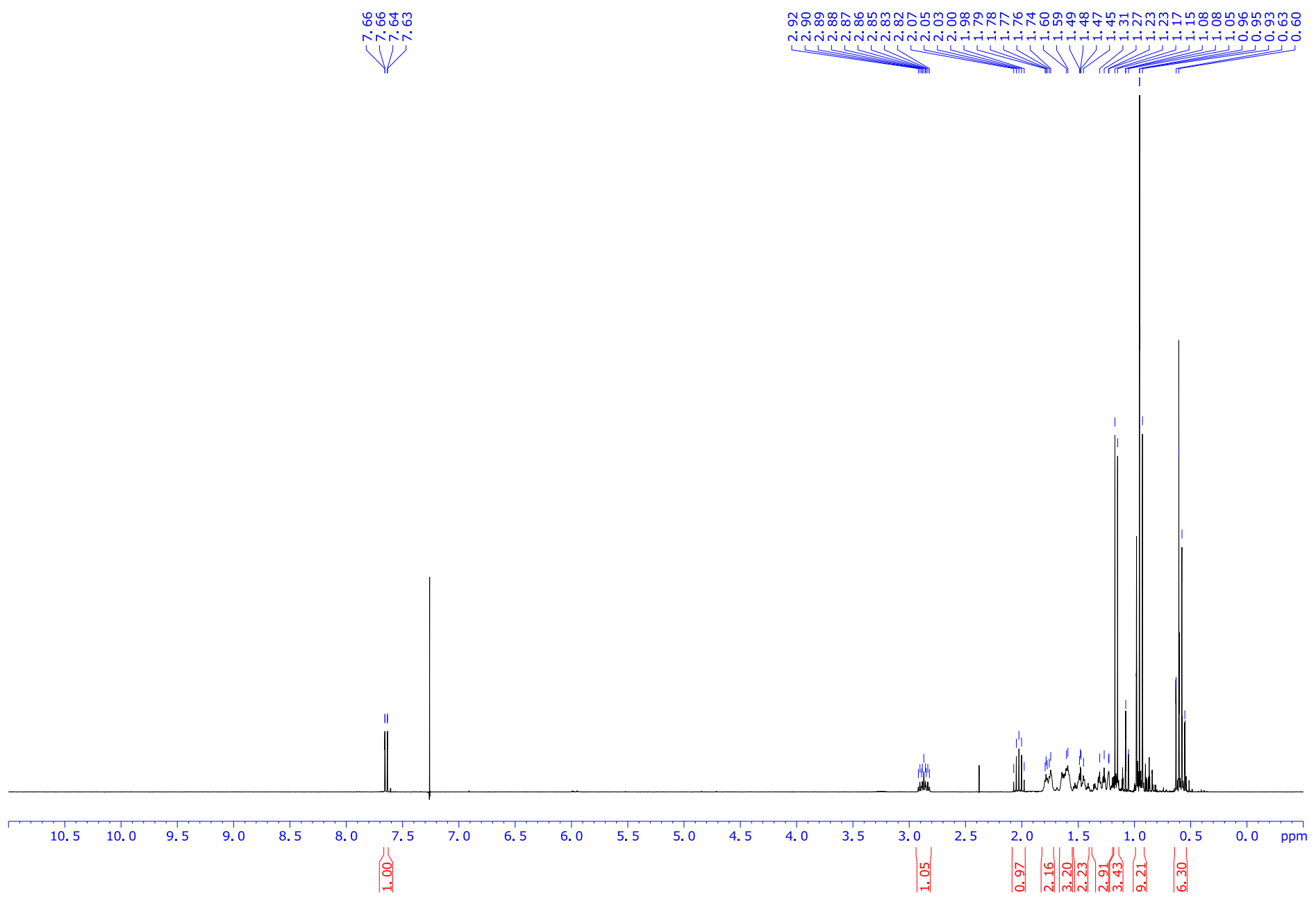




\section{$(4 R, 6 R, E)-2,4,6-T$ rimethyloct-2-enal $[(R, R)-10]$}

and

\section{$(4 S, 6 S, E)-2,4,6-$ Trimethyloct-2-enal $[(S, S)-10]$}

${ }^{1} \mathbf{H}$ NMR (500.10 MHz, $\left.\mathrm{CDCl}_{3}\right)$ :

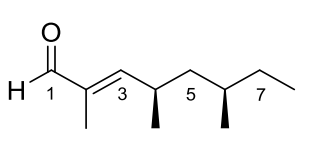

$(R, R)-10$

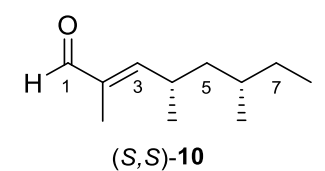

$(S, S)-10$

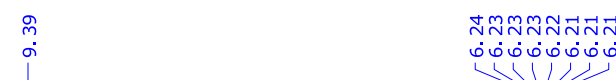

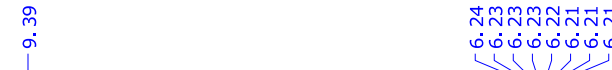

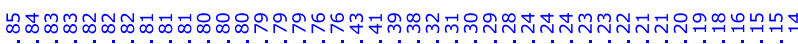

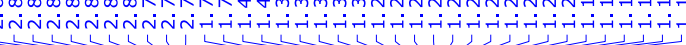

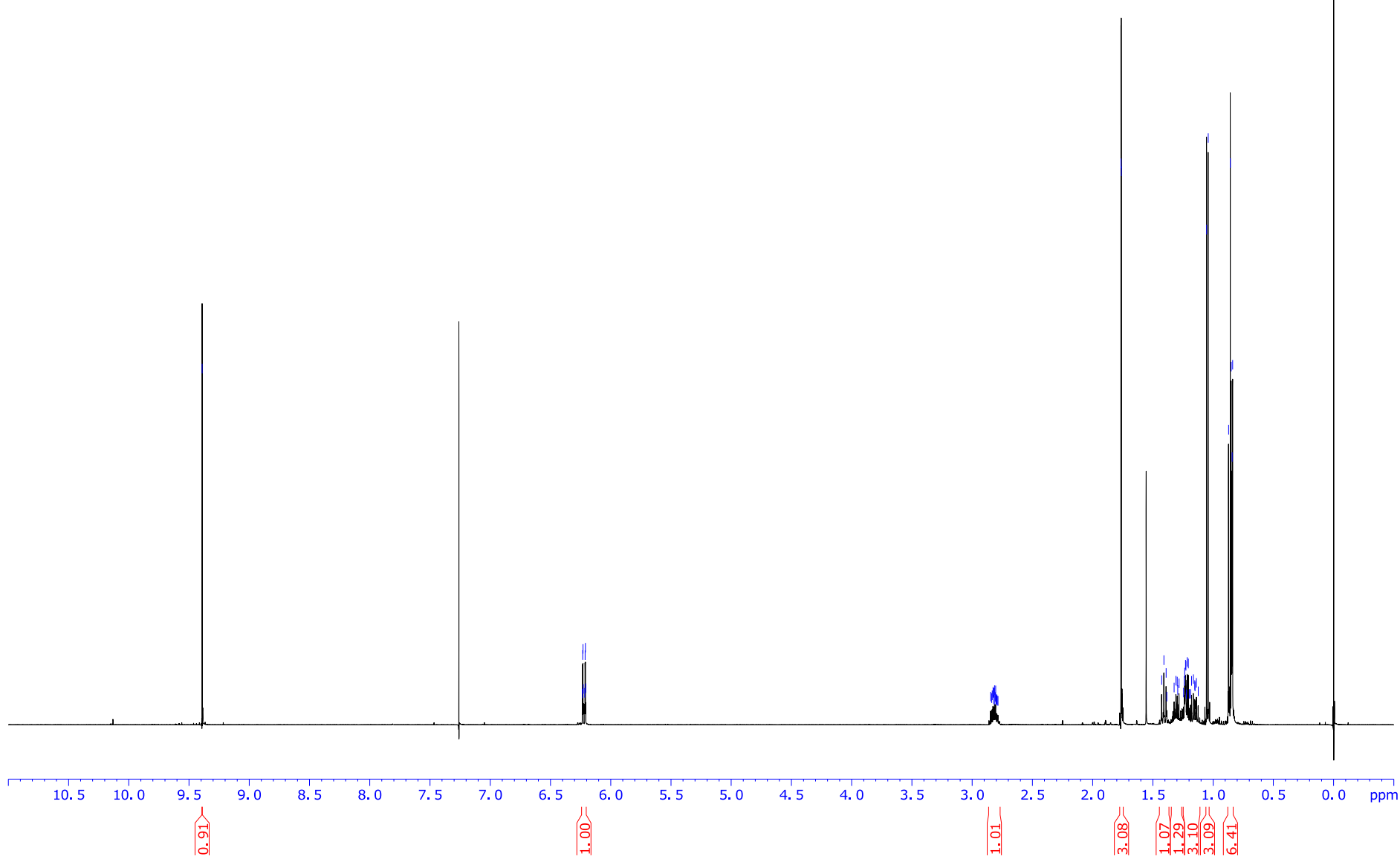




\section{$(4 R, 6 R, E)-2,4,6$-trimethyloct-2-enal $[(R, R)-10]$}

and

\section{$(4 S, 6 S, E)-2,4,6$-trimethyloct-2-enal $[(S, S)-10]$}

${ }^{13}$ C NMR (125.75 MHz, $\left.\mathrm{CDCl}_{3}\right)$ :

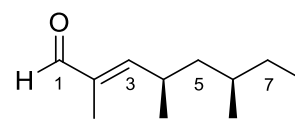

$(R, R)-10$
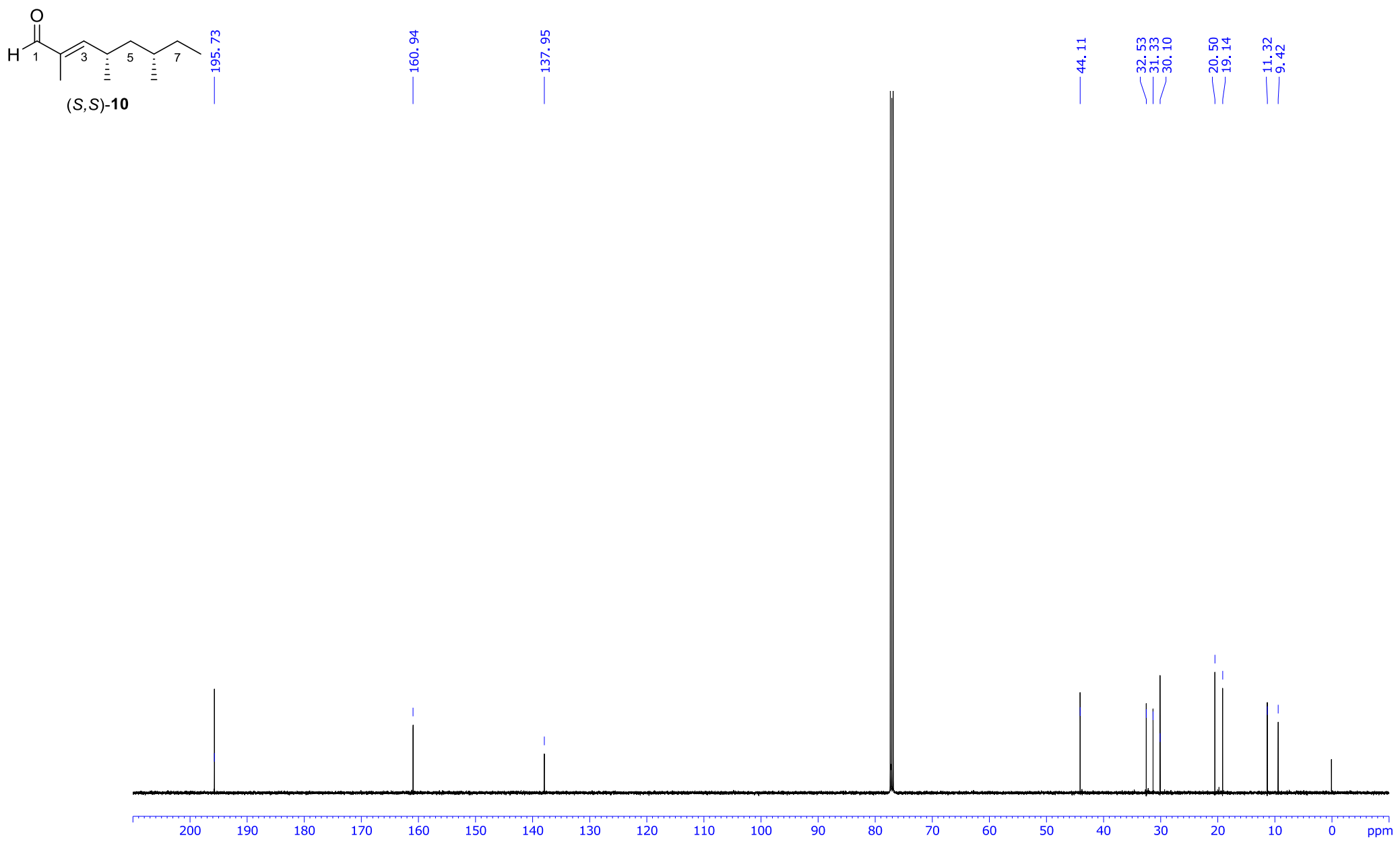


\section{Dimethyl (2-Oxopropyl)phosphonate (SI-3)}

${ }^{1}$ H NMR (300.13 MHz, $\left.\mathrm{CDCl}_{3}\right)$ :
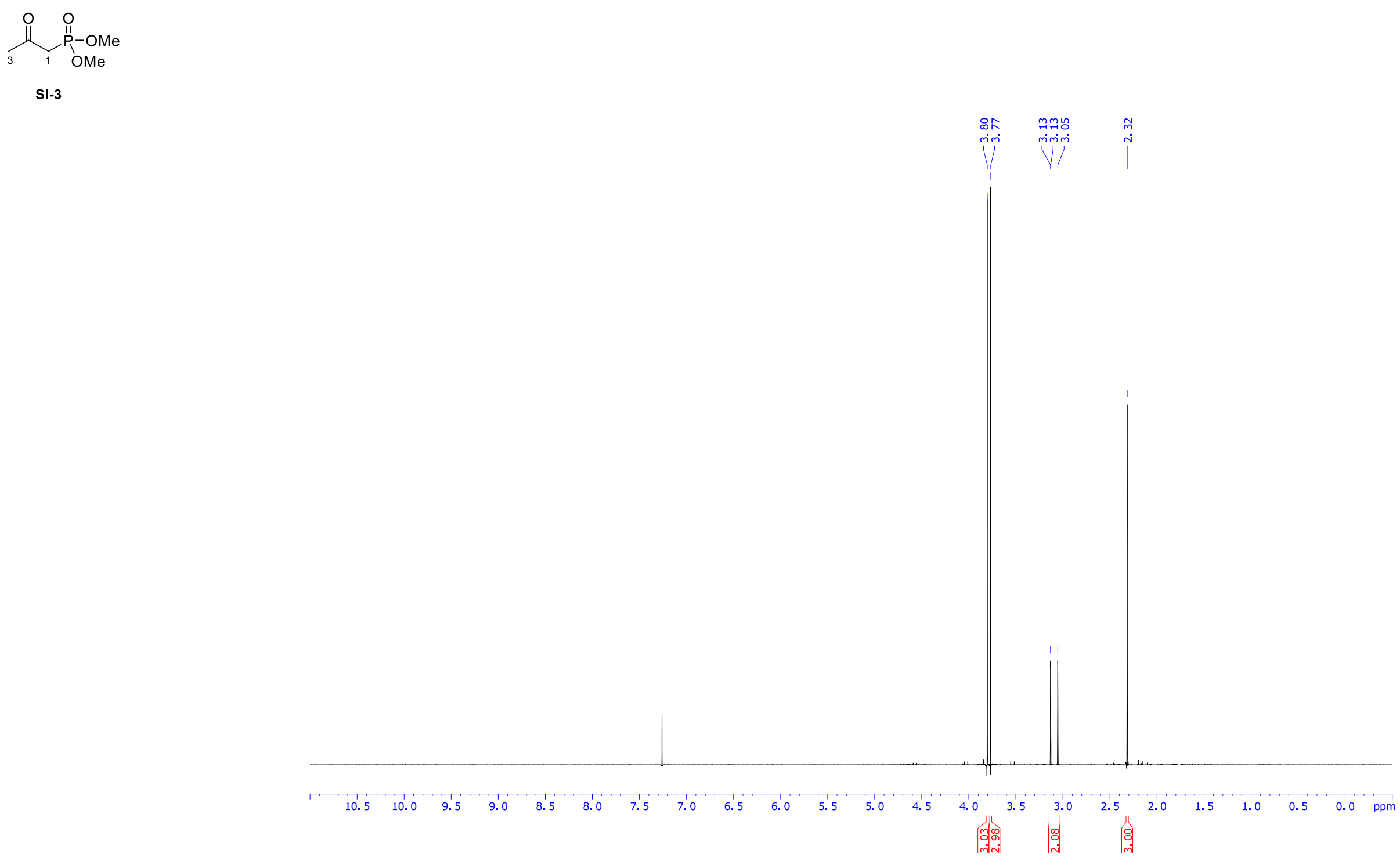


\section{Dimethyl (1-Diazo-2-oxopropyl)phosphonate (SI-4)}

${ }^{1}$ H NMR (300.13 MHz, $\left.\mathrm{CDCl}_{3}\right)$ :
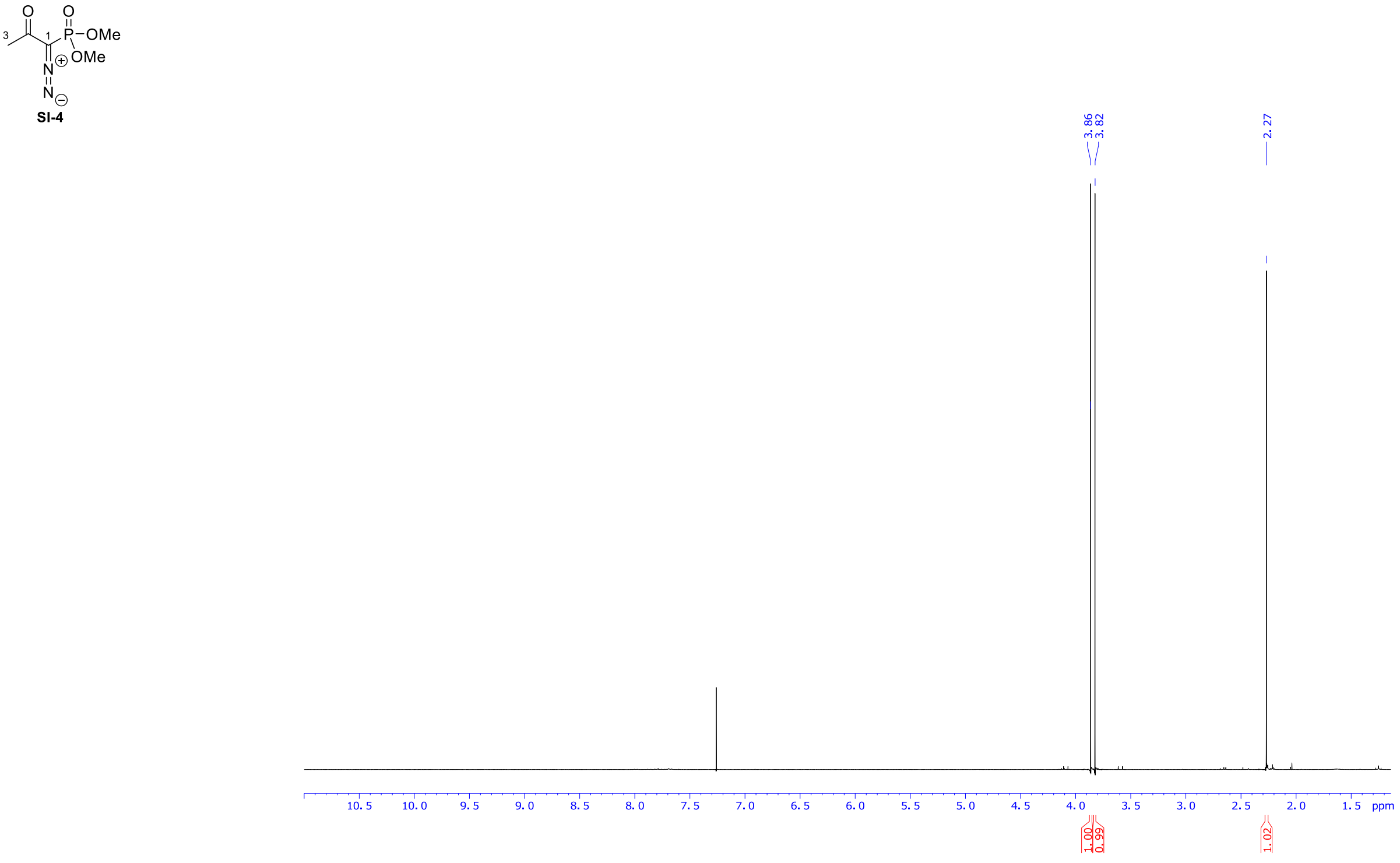


\section{Dimethyl (Diazomethyl)phosphonate (31)}

${ }^{1}$ H NMR (300.13 MHz, $\left.\mathrm{CDCl}_{3}\right)$ :

$$
\begin{aligned}
& \text { 1. } \\
& \prod_{\oplus} \mathrm{OMe} \\
& \mathrm{N}_{\ominus}
\end{aligned}
$$

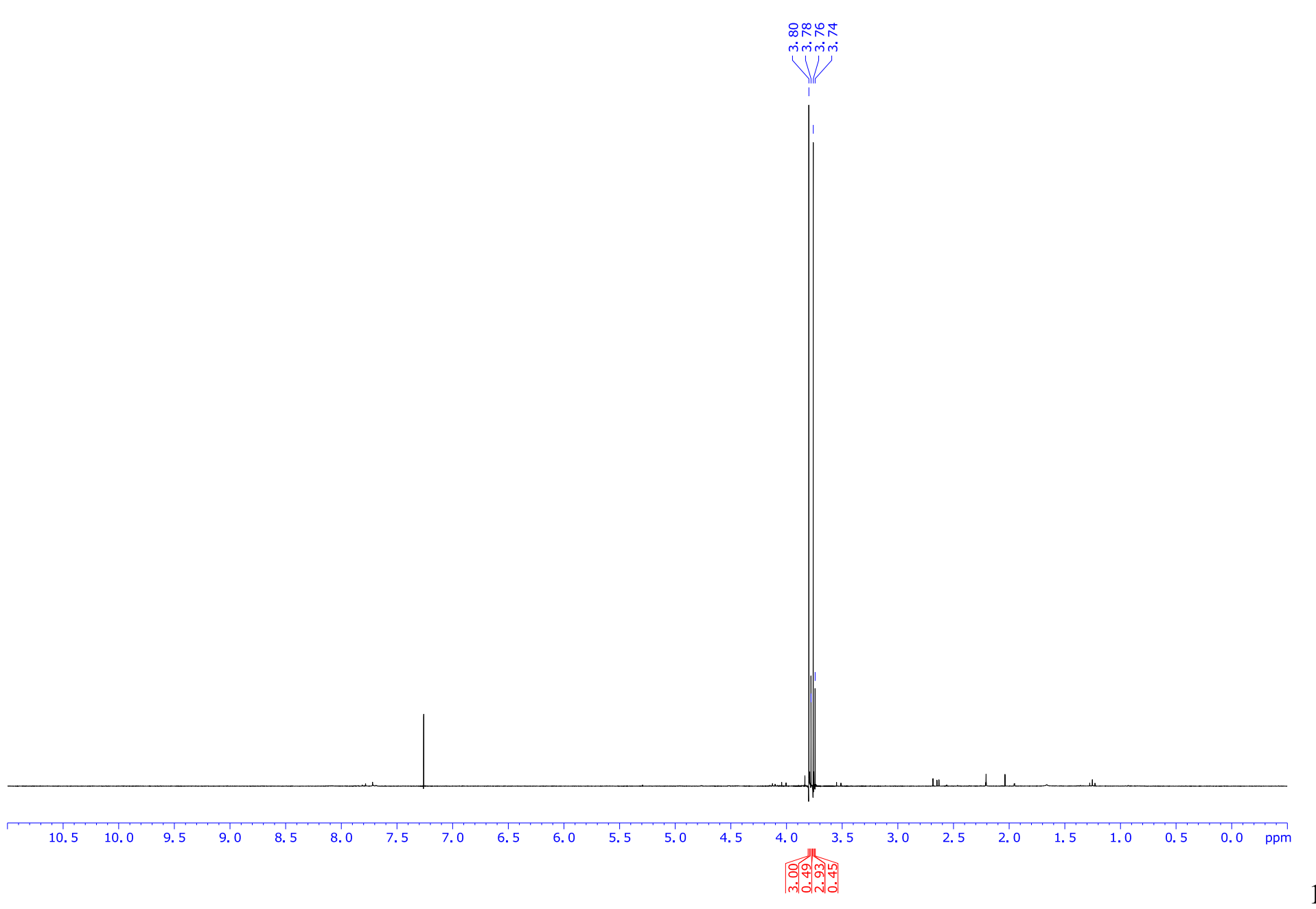


$(5 R, 7 R, E)-3,5,7-T r i m e t h y l n o n-3-e n-1-y n e ~[(R, R)-32]$

and

$(5 S, 7 S, E)-3,5,7-T r i m e t h y l n o n-3-e n-1-y n e ~[(S, S)-32]$

${ }^{1} \mathbf{H}$ NMR (500.10 MHz, $\left.\mathrm{CDCl}_{3}\right)$ :
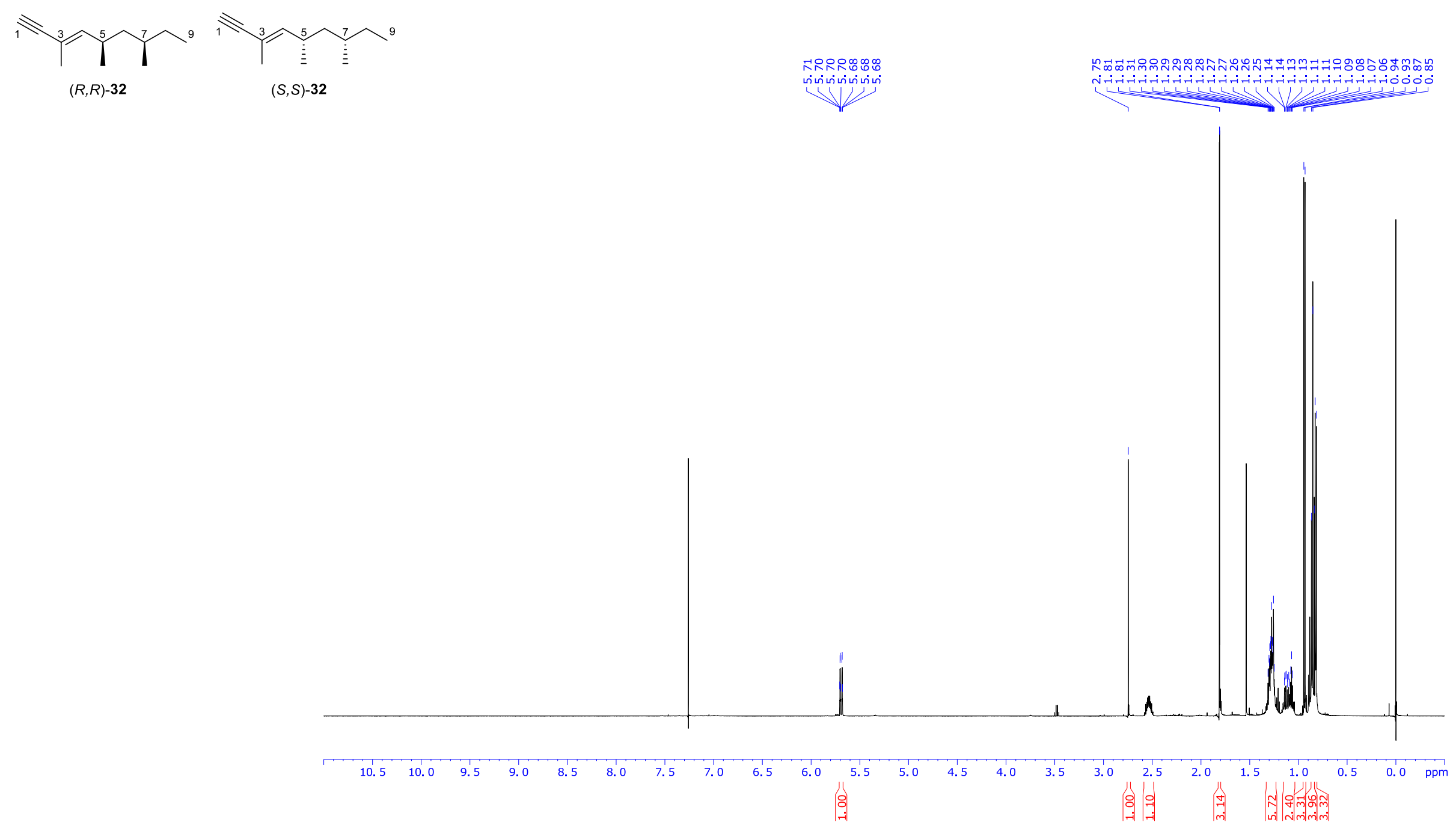
$(5 R, 7 R, E)-3,5,7-t r i m e t h y l n o n-3-e n-1-y n e ~[(R, R)-32]$

and

$(5 S, 7 S, E)$-3,5,7-trimethylnon-3-en-1-yne [( $S, S)$-32]

${ }^{13}$ C NMR (125.75 MHz, $\left.\mathrm{CDCl}_{3}\right)$ :

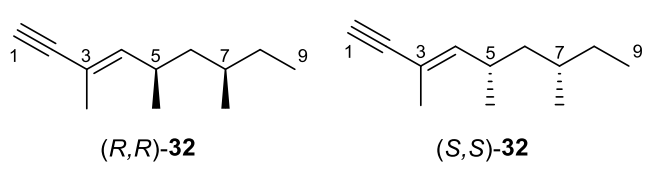

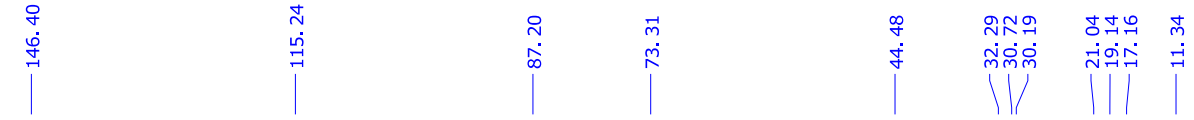

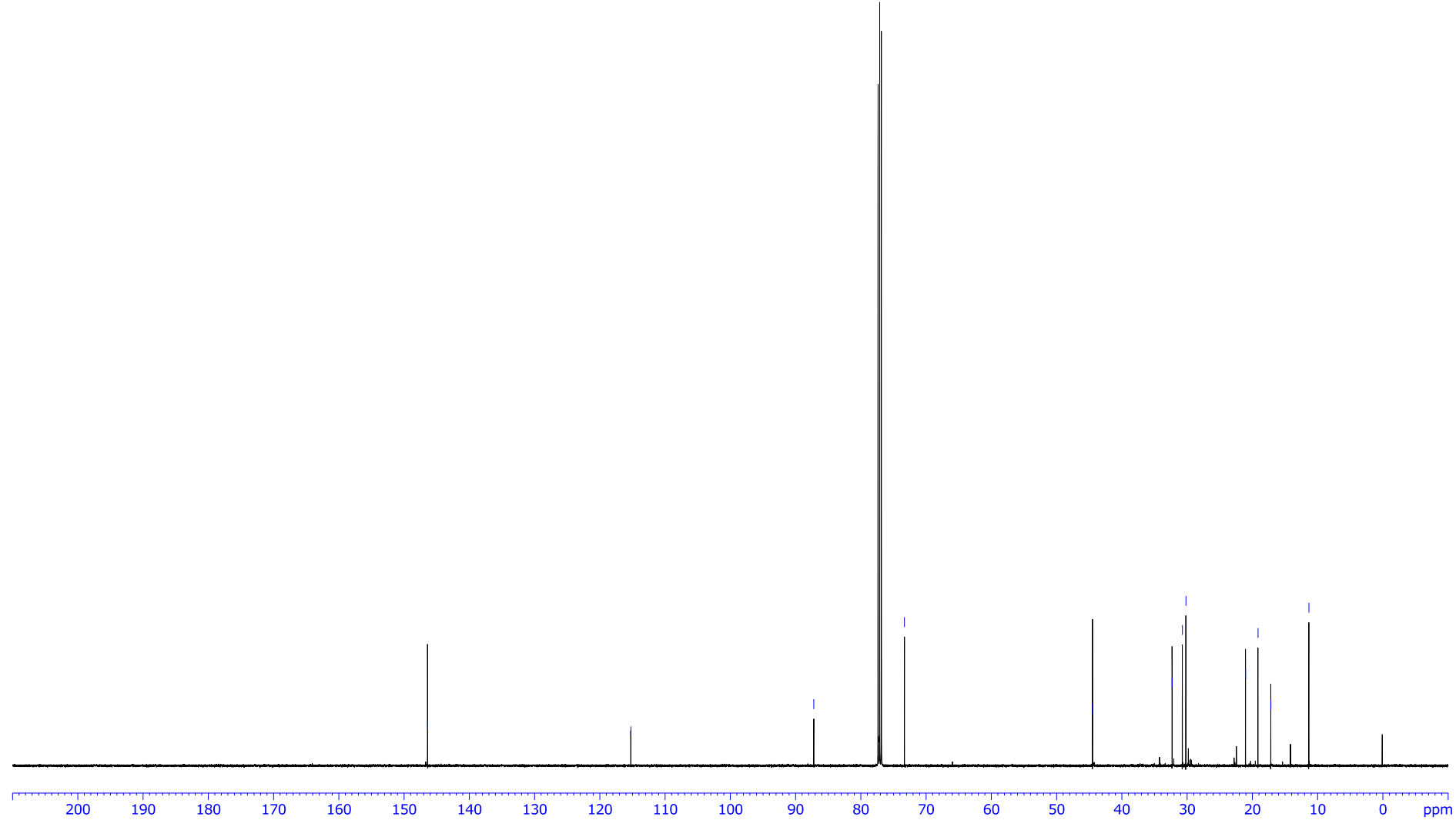


Christian Drescher, Morris Keller, Olivier Potterat, Matthias Hamburger and Reinhard Brückner, Structure-Elucidating Total Synthesis of the (Polyenoyl)tetramic Acid Militarinone C

3.3 NMR Spectra of all 4 Diastereomeres of the Tetramic Acid Militarionone C (1) 
Methyl (2'S)-2'- $\{N$-[(1E,2Z,4E,6E,8E,10S,12S)-3-Hydroxy-8,10,12-trimethyltetradeca-2,4,6,8-tetraenamido]- $N$-(1'"',5"'-dimethoxybenzyl) $\}-$ $3 '-\left\{1^{\prime \prime}-[(\right.$ tert-butyldimethylsilyl)oxy]phenyl $\}$ propanoate $[E-e n o l-(S, S, S)-33]$

and

Methyl $(2 ' R)-2$ '- $\{N$-[(1E,2Z,4E,6E,8E,10R,12R)-3-Hydroxy-8,10,12-trimethyltetradeca-2,4,6,8-tetraenamido]- $N$-(1'"',5'"-dimethoxybenzyl) $\}$ $3 '-\left\{1^{\prime \prime}-[(\right.$ tert-butyldimethylsilyl)oxy]phenyl $\}$ propanoate $[E$-enol-(R,R,R)-33]

${ }^{1} \mathbf{H}$ NMR $\left(500.10 \mathrm{MHz}, \mathrm{CDCl}_{3}\right)$ :
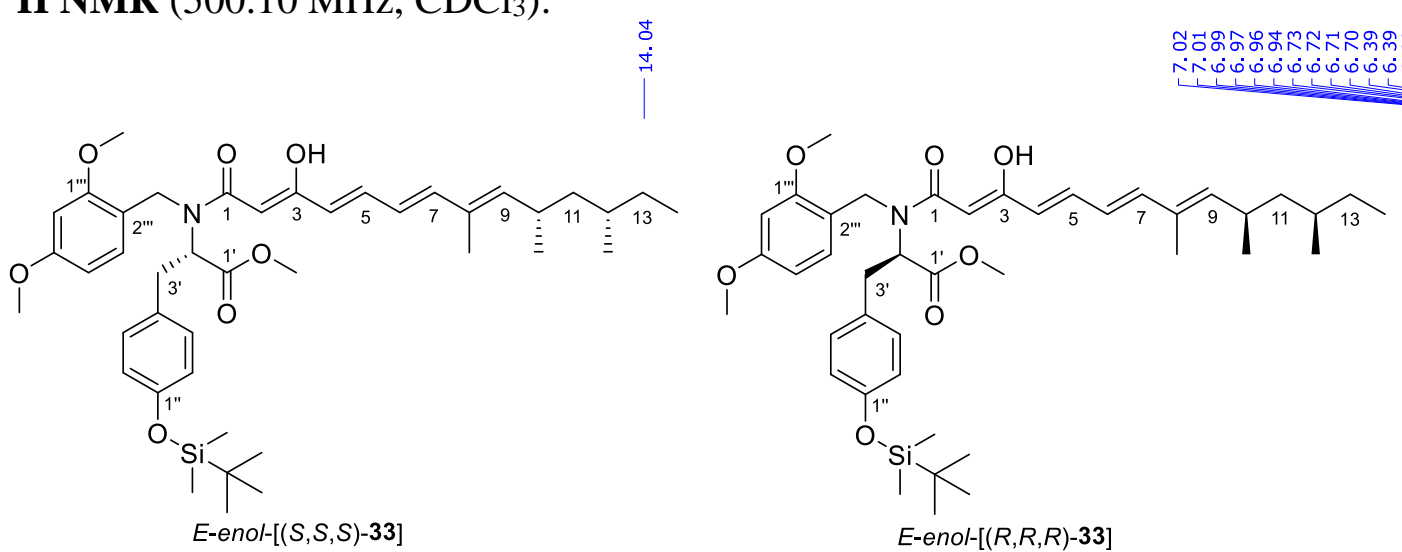

E-enol-[(S,S,S)-33)

E-enol-[(R,R,R)-33]

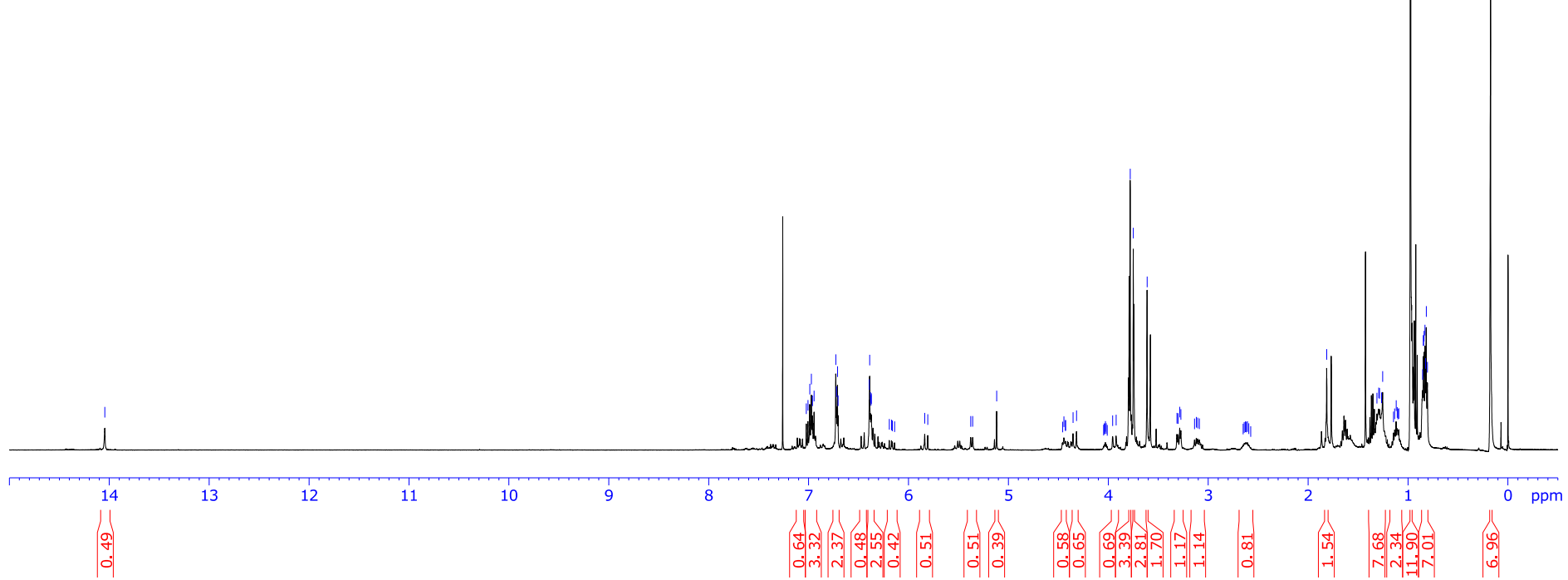


Methyl (2'S)-2'- $\{N$-[(1E,2Z,4E,6E,8E,10S,12S)-3-Hydroxy-8,10,12-trimethyltetradeca-2,4,6,8-tetraenamido]- $N$-(1'"',5"'-dimethoxybenzyl) $\}$ $3 '-\left\{1^{\prime \prime}-[(\right.$ tert-butyldimethylsilyl)oxy]phenyl $\}$ propanoate $[E-e n o l-(S, S, S)-33]$

and

Methyl (2'R)-2'- $\{N$-[(1E,2Z,4E,6E,8E,10R,12R)-3-Hydroxy-8,10,12-trimethyltetradeca-2,4,6,8-tetraenamido]- $N$-(1'"',5'"'-dimethoxybenzyl) $\}$ $3 '-\left\{1^{\prime \prime}-[(\right.$ tert-butyldimethylsilyl)oxy]phenyl $\}$ propanoate $[E$-enol-(R,R,R)-33]

${ }^{13} \mathrm{C}$ NMR $\left(125.75 \mathrm{MHz}, \mathrm{CDCl}_{3}\right)$
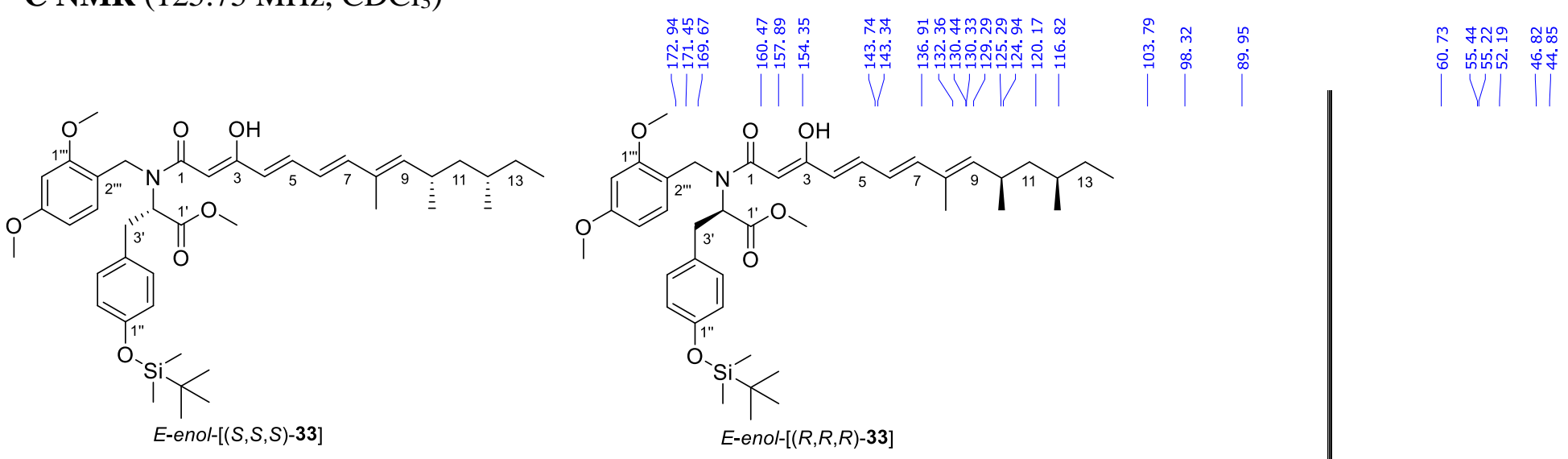

E-enol-[(R,R,R)-33] 
Methyl (2'S)-2'- $\{N-[(1 E, 4 E, 6 E, 8 E, 10 S, 12 S)-3-O x 0-8,10,12$-trimethyltetradeca-4,6,8-trienamido]- $N$-(1'"',5'"'-dimethoxybenzyl) $\}-3$ '- $\{1$ "'-[(tertbutyldimethylsilyl)oxy]phenyl $\}$ propanoate $[E-k e t o-(S, S, S)-33]$

$$
\text { and }
$$

Methyl (2'R)-2'- $\{N$-[(1E,4E,6E,8E,10R,12R)-3-Oxo-8,10,12-trimethyltetradeca-4,6,8-trienamido]- $N$-(1"',5"'-dimethoxybenzyl) $\}-3$ '- $\{1$ "'[(tert-butyldimethylsilyl)oxy $]$ phenyl $\}$ propanoate $[$ E-keto- $(R, R, R)-33]$

${ }^{1} \mathbf{H}$ NMR $\left(500.10 \mathrm{MHz}, \mathrm{CDCl}_{3}\right)$ :

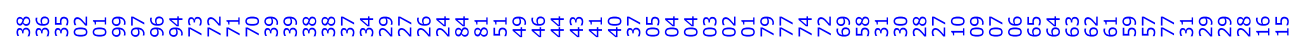
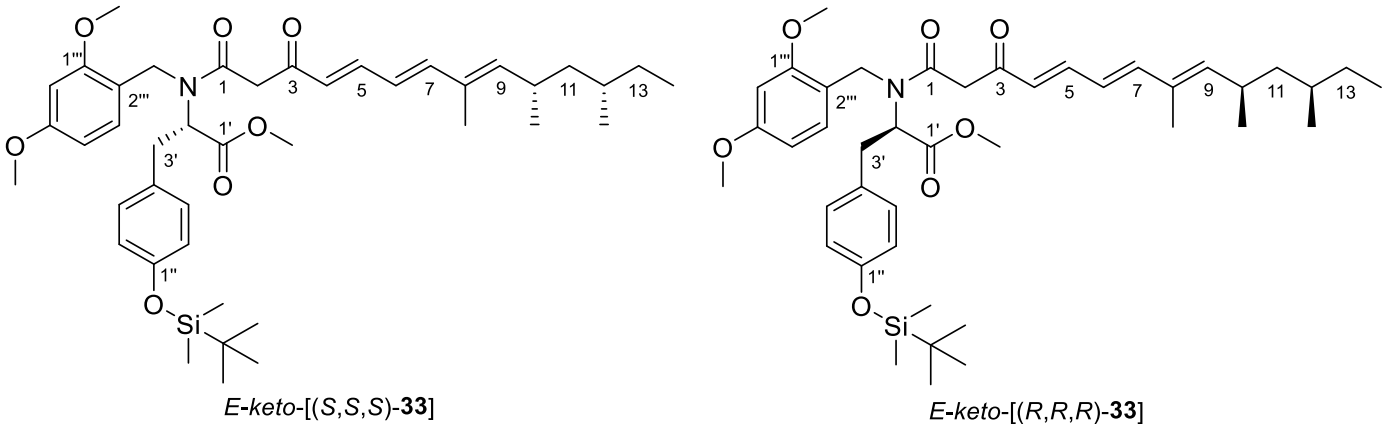

E-keto-[(R,R,R)-33]

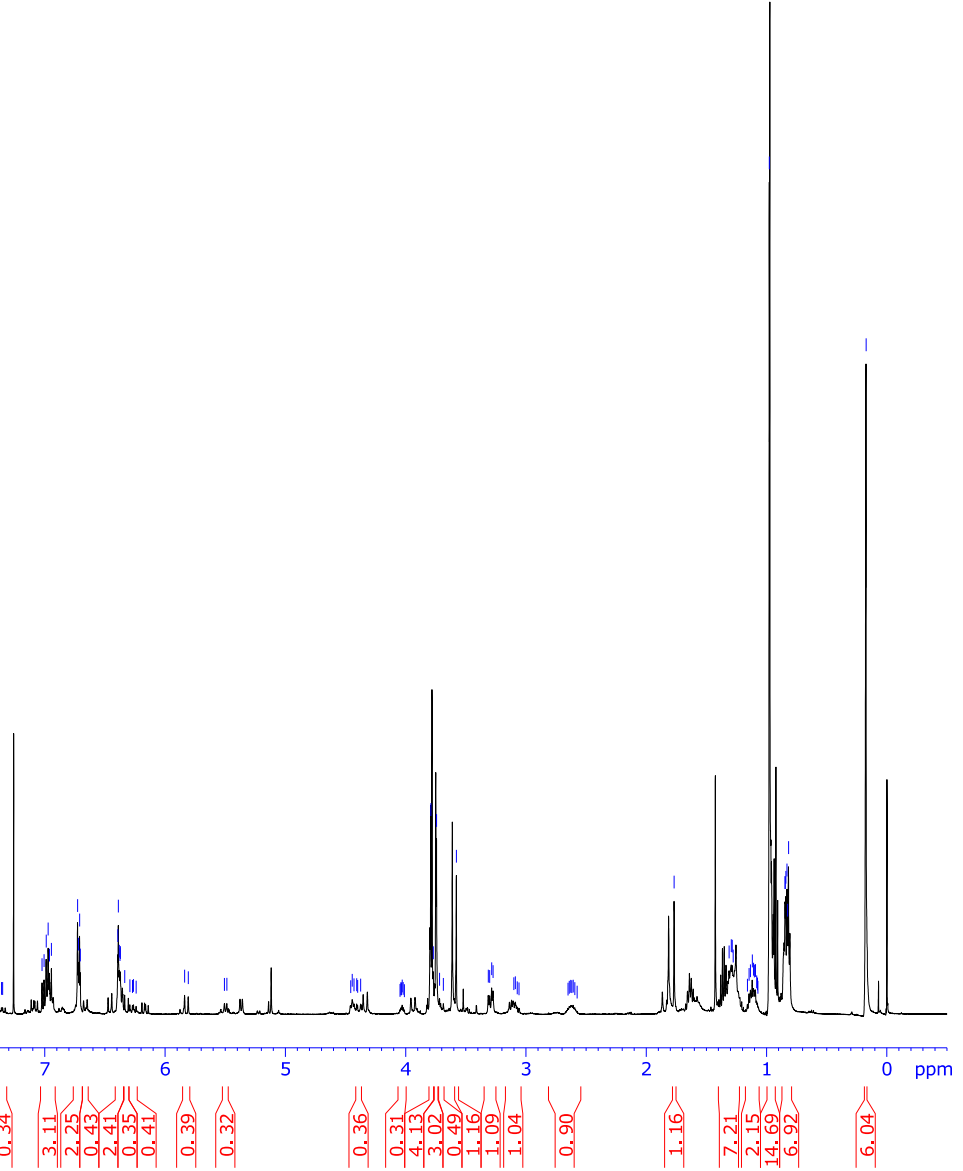


Methyl (2'S)-2'- $\{N$-[(1E,4E,6E,8E,10S,12S)-3-Oxo-8,10,12-trimethyltetradeca-4,6,8-trienamido]- $N$-(1'"',5'"'-dimethoxybenzyl) $\}-3$ '- $\{1$ "'-[(tertbutyldimethylsilyl)oxy]phenyl $\}$ propanoate $[E-k e t o-(S, S, S)-33]$

$$
\text { and }
$$

Methyl (2'R)-2'- $\{N$-[(1E,4E,6E,8E,10R,12R)-3-Oxo-8,10,12-trimethyltetradeca-4,6,8-trienamido]- $N$-(1"',5'"'-dimethoxybenzyl) $\}-3$ '- $\{1$ "'[(tert-butyldimethylsilyl)oxy]phenyl $\}$ propanoate $[$ E-keto- $(R, R, R)-33]$

${ }^{13} \mathrm{C}$ NMR (125.75 MHz, $\left.\mathrm{CDCl}_{3}\right)$ :

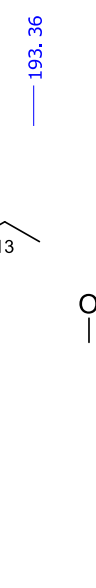

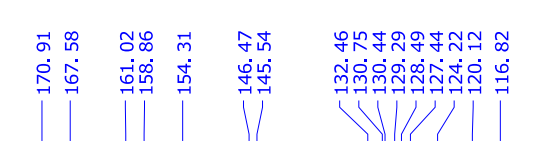
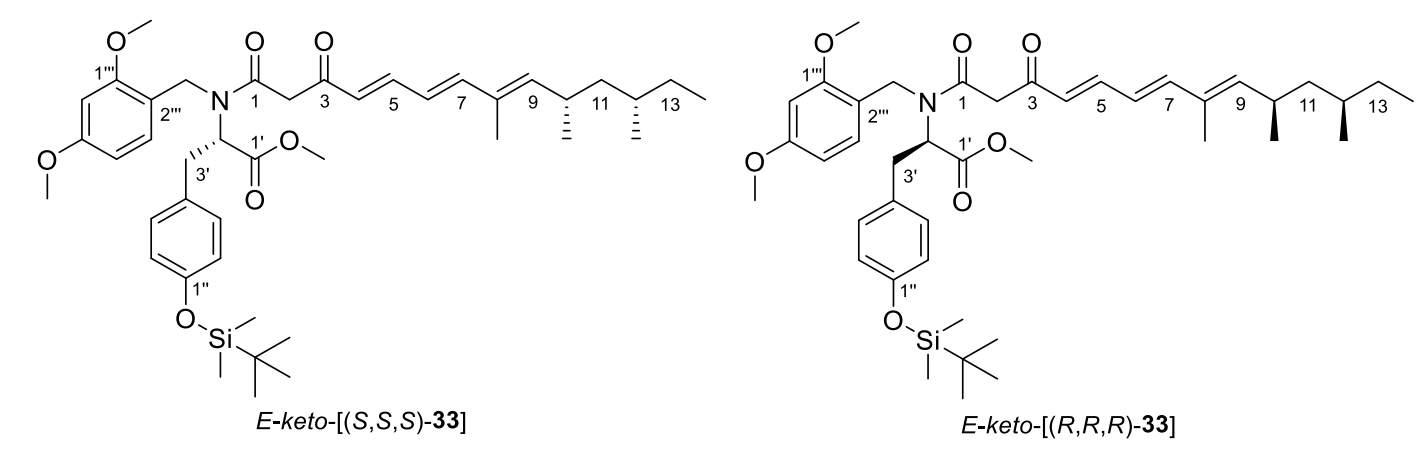

E-keto-[(R,R,R)-33]
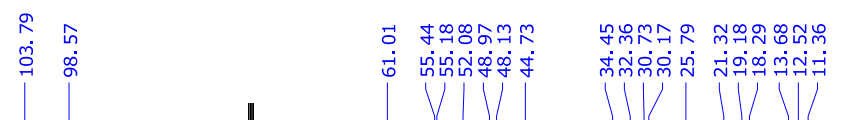

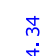

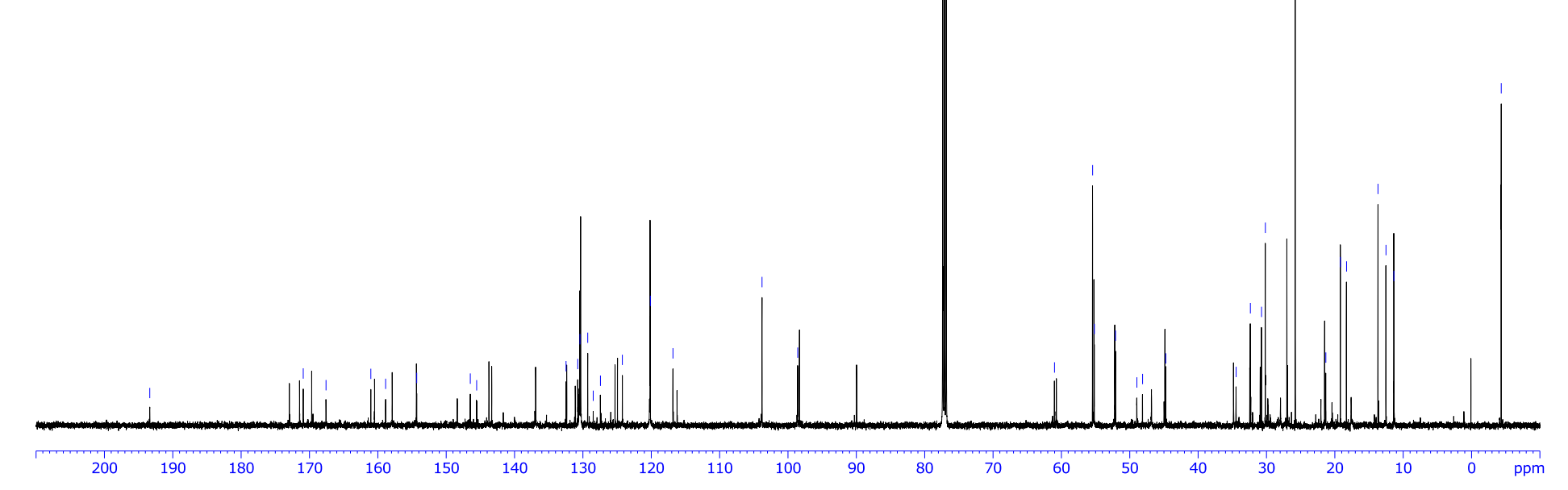


Methyl (2'S)-2'- $\{N-[(1 E, 2 Z, 4 E, 6 E, 8 E, 10 R, 12 R)-3-H y d r o x y-8,10,12$-trimethyltetradeca-2,4,6,8-tetraenamido]- $N$-(1"',5'"'-dimethoxybenzyl) $\}$ 3 '- $\{1$ "-[(tert-butyldimethylsilyl)oxy]phenyl $\}$ propanoate $[E$-enol-(S,R,R)-33]

and

Methyl (2'R)-2'- $\{N-[(1 E, 2 Z, 4 E, 6 E, 8 E, 10 S, 12 S)-3-H y d r o x y-8,10,12-t r i m e t h y l t e t r a d e c a-2,4,6,8-t e t r a e n a m i d o]-N-(1 ' ", 5 ' " '-d i m e t h o x y b e n z y l)\}-$ $3^{\prime}-\left\{1^{\prime \prime}-[(\right.$ tert-butyldimethylsilyl)oxy]phenyl $\}$ propanoate $[E-e n o l-(R, S, S)-33]$

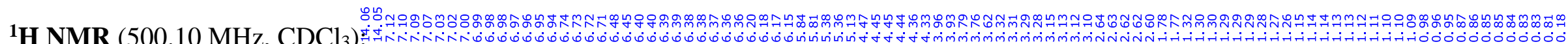<smiles>CCC(C)C=C(C)/C=C(C)/C=C/C=C/C(O)=C/C(=O)N(Cc1ccc(OC)cc1OC)[C@H](Cc1ccc(O[Si](C)(C)C(C)(C)C)cc1)C(=O)OC</smiles>

E-enol-[(S,R,R)-33]
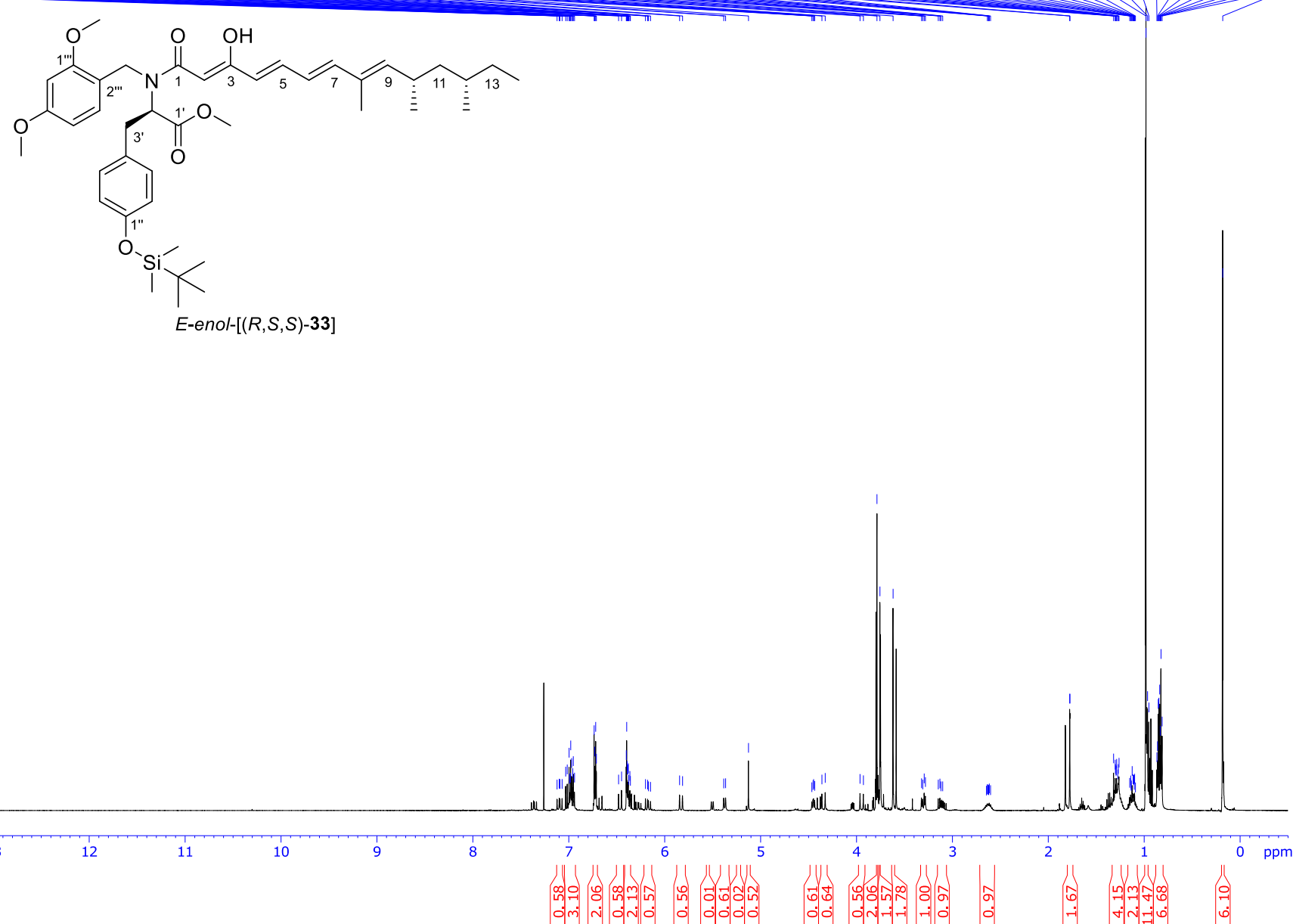
Methyl (2'S)-2'- $\{N-[(1 E, 2 Z, 4 E, 6 E, 8 E, 10 R, 12 R)-3-H y d r o x y-8,10,12$-trimethyltetradeca-2,4,6,8-tetraenamido]- $N$-(1"',5'"'-dimethoxybenzyl) $\}$ 3 '- $\{1$ "-[(tert-butyldimethylsilyl)oxy]phenyl $\}$ propanoate $[E$-enol-(S,R,R)-33]

and

Methyl (2' $R)-2$ '- $\{N-[(1 E, 2 Z, 4 E, 6 E, 8 E, 10 S, 12 S)-3-H y d r o x y-8,10,12-t r i m e t h y l t e t r a d e c a-2,4,6,8-t e t r a e n a m i d o]-N-(1 ' ", 5 ' " '-d i m e t h o x y b e n z y l)\}-$ $3^{\prime}-\left\{1^{\prime \prime}-[(\right.$ tert-butyldimethylsilyl)oxy]phenyl $\}$ propanoate $[E$-enol-(R,S,S)-33]

${ }^{13}$ C NMR (125.75 MHz, $\left.\mathrm{CDCl}_{3}\right)$ :
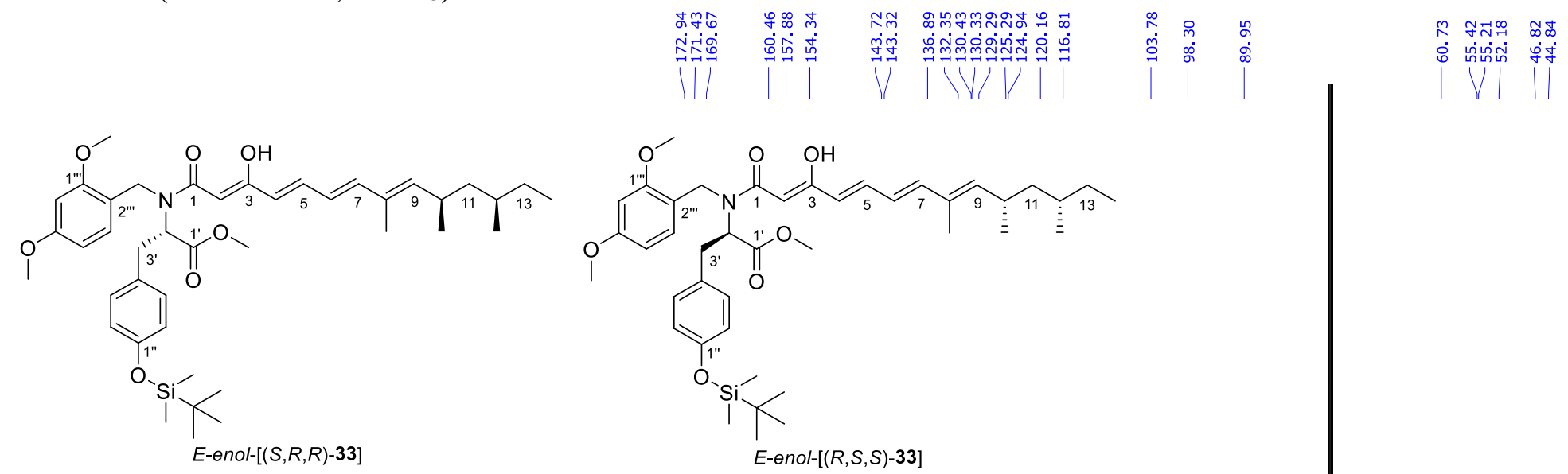

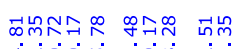

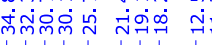

E-enol-[(R,S,S)-33]

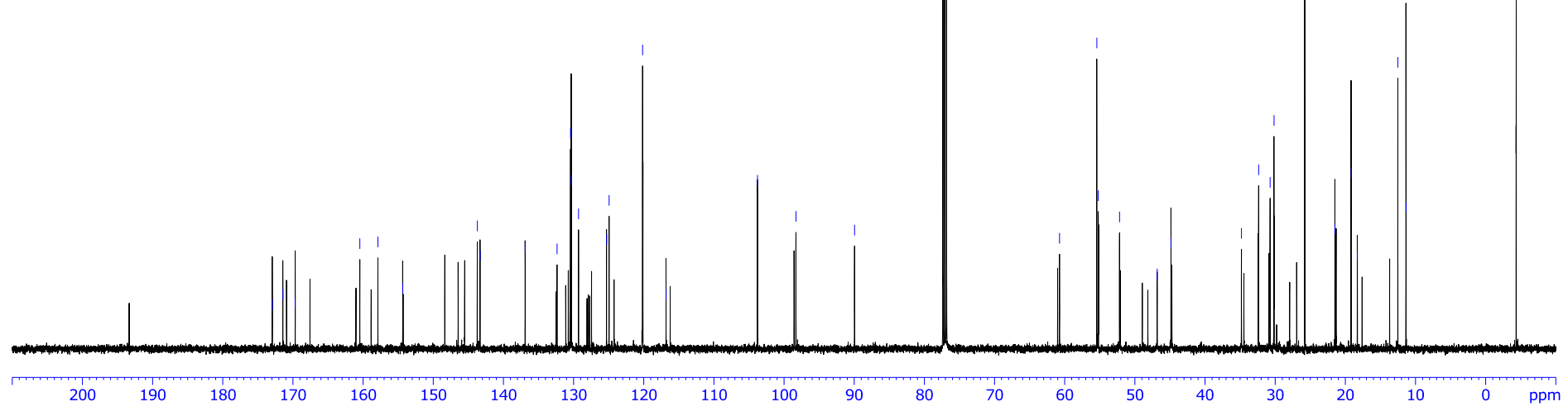


Methyl (2'S)-2'- $\{N$-[(1E,4E,6E,8E,10R,12R)-3-Oxo-8,10,12-trimethyltetradeca-4,6,8-trienamido]- $N$-(1'"',5'"'-dimethoxybenzyl) $\}-3$ '- $\{1$ '"[(tert-butyldimethylsilyl)oxy]phenyl $\}$ propanoate $[E-k e t o-(S, R, R)-33]$

and

Methyl (2'R)-2'- $\{N$-[(1E,4E,6E,8E,10S,12S)-3-Oxo-8,10,12-trimethyltetradeca-4,6,8-trienamido]- $N$-(1"',5'"'-dimethoxybenzyl $)\}-3$ '- $\{1 "$ '-[(tertbutyldimethylsilyl)oxy]phenyl $\}$ propanoate $[$ E-keto- $(R, S, S)-33]$

${ }^{1} \mathbf{H}$ NMR (500.10 MHz, $\left.\mathrm{CDCl}_{3}\right)$ :

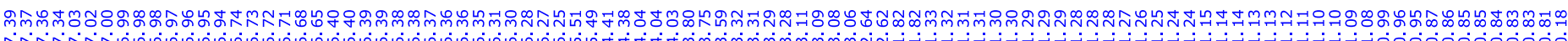

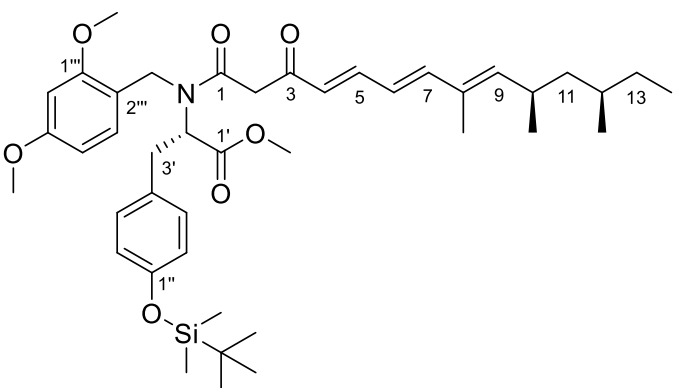

E-keto-[(S,R,R)-33]

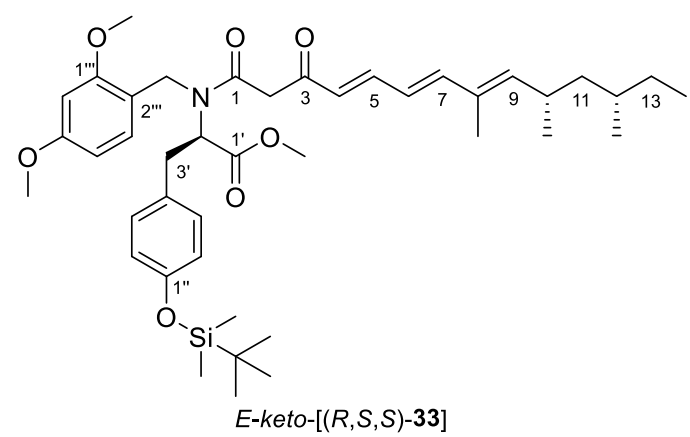

E-keto-[(R,S,S)-33]

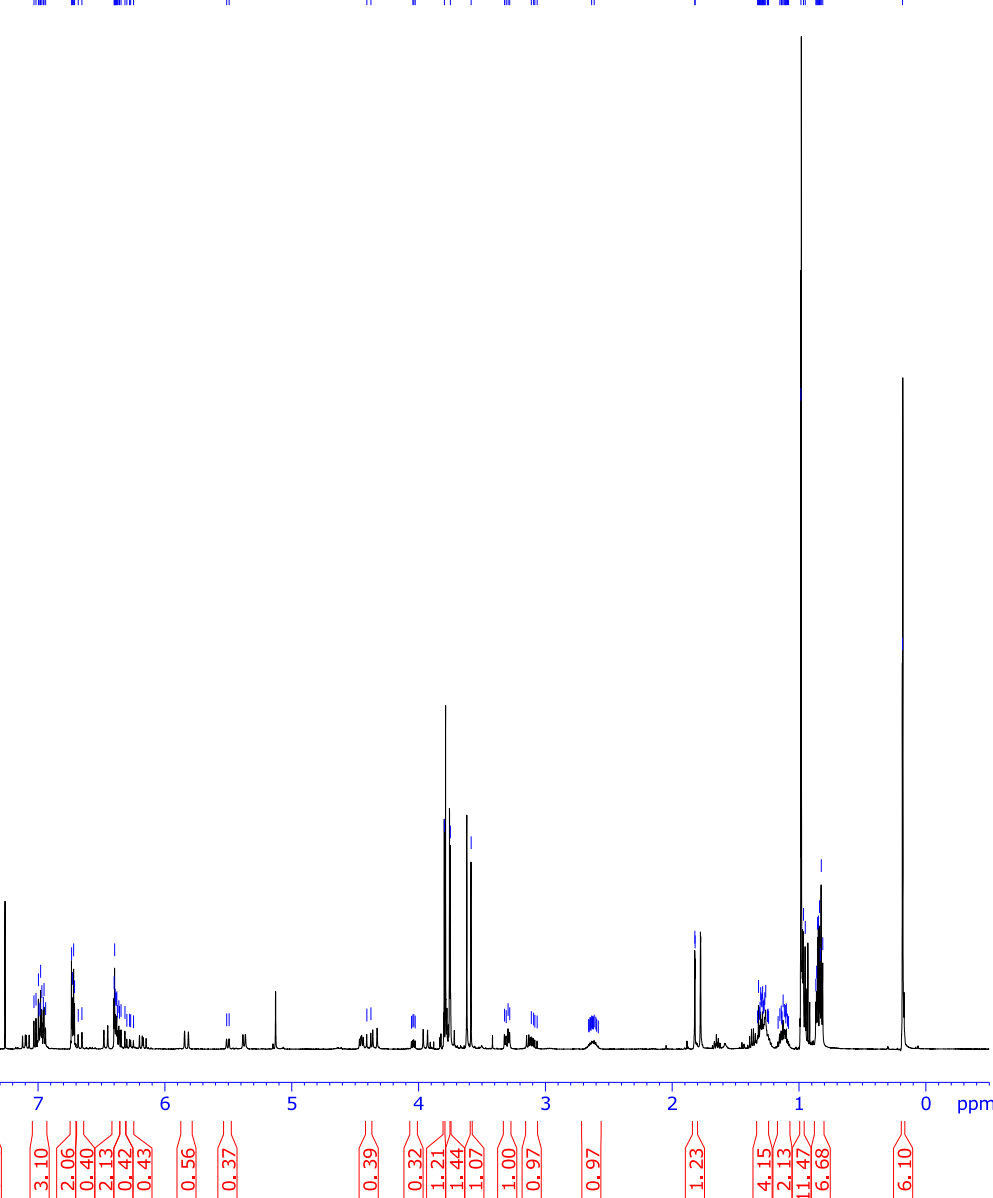


Methyl (2'S)-2'- $\{N$-[(1E,4E,6E,8E,10R,12R)-3-Oxo-8,10,12-trimethyltetradeca-4,6,8-trienamido]- $N$-(1'"',5'"'-dimethoxybenzyl) $\}-3$ '- $\{1$ '"[(tert-butyldimethylsilyl)oxy]phenyl $\}$ propanoate $[E-k e t o-(S, R, R)-33]$

and

Methyl (2'R)-2'- $\{N$-[(1E,4E,6E,8E,10S,12S)-3-Oxo-8,10,12-trimethyltetradeca-4,6,8-trienamido]- $N$-(1"',5'"'-dimethoxybenzyl $\}$-3'- $\{1 "$ "-[(tertbutyldimethylsilyl)oxy]phenyl $\}$ propanoate $[$ E-keto- $(R, S, S)-33]$

${ }^{13}$ C NMR (125.75 MHz, $\left.\mathrm{CDCl}_{3}\right)$ :
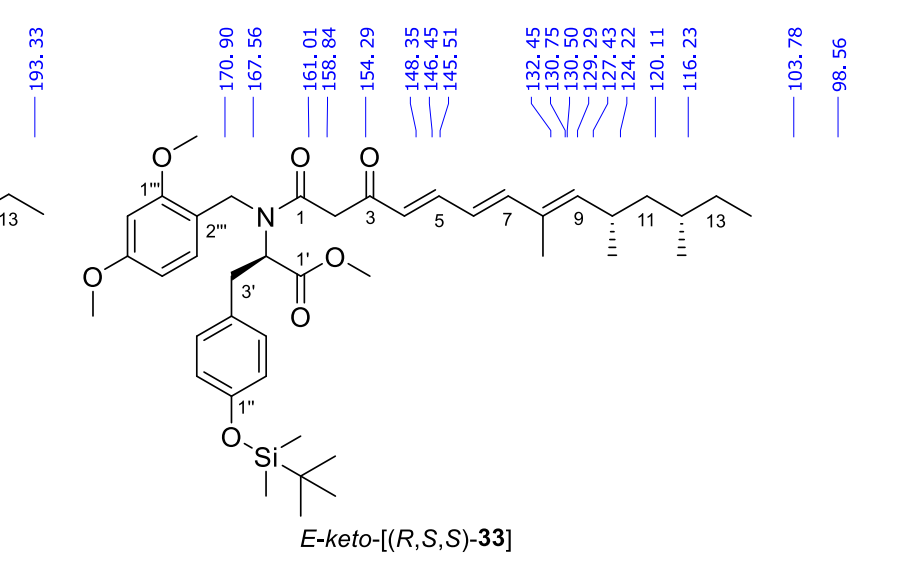

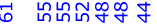

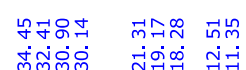

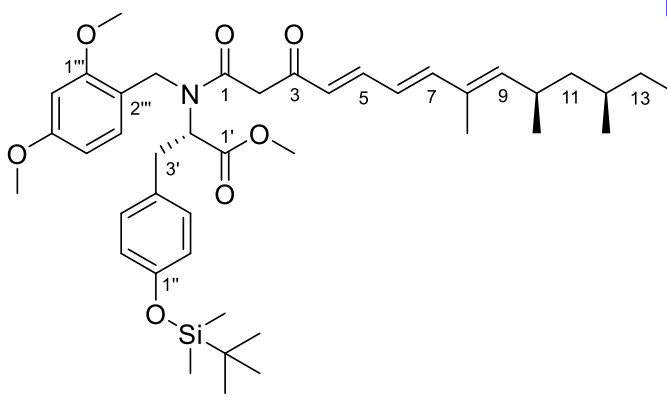

E-keto-[(S,R,R)-33]

E-keto-[(R,S,S)-33]

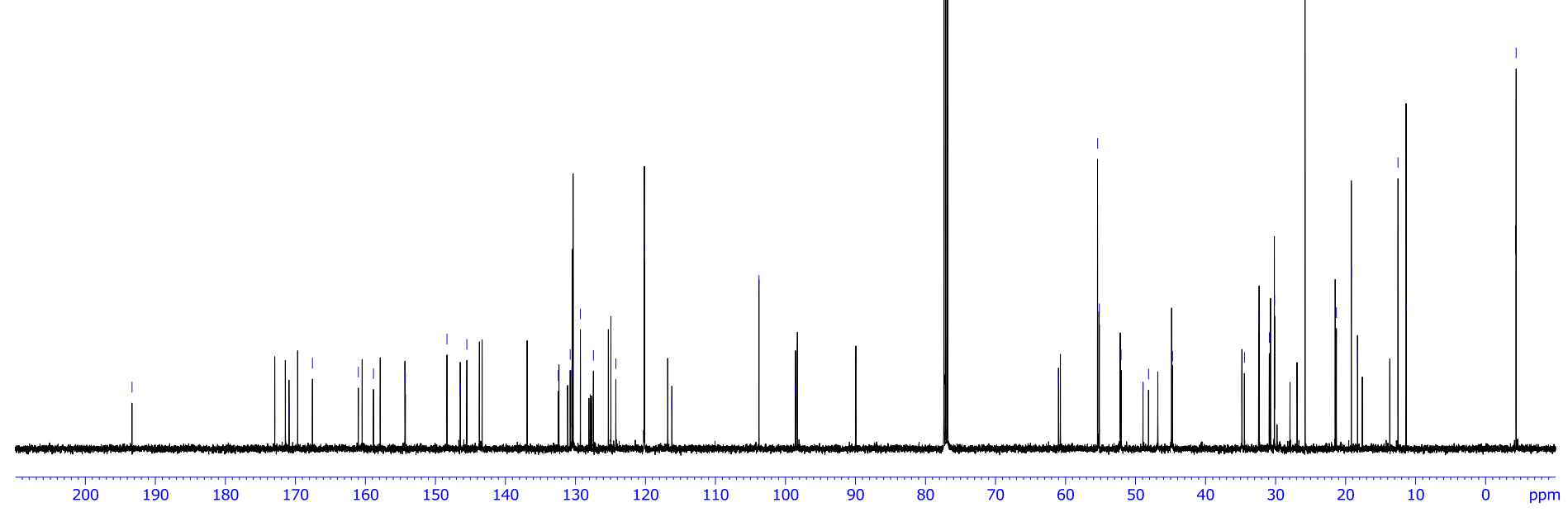


(5S)-5-(1'-Hydroxybenzyl)-1-(1'"',5'"'-dimethoxybenzyl)-3-(1'Z,2' $E, 4^{\prime} E, 6^{\prime} E, 8^{\prime} S, 10$ 'S)-1'-hydroxy-6',8',10'-trimethyldodeca-2',4',6'-trien-1'ylidene)pyrrolidine-2,4-dion [Z-enol-(S,S,S)-34]

and

(5R)-5-(1"'-Hydroxybenzyl)-1-(1'"',5'"'-dimethoxybenzyl)-3-(1'Z,2' $\left.E, 4^{\prime} E, 6^{\prime} E, 8^{\prime} R, 10^{\prime} R\right)-1^{\prime}$-hydroxy-6', $8^{\prime}, 10^{\prime}$-trimethyldodeca-2' $4^{\prime}, 6^{\prime}$-trien-1'ylidene)pyrrolidine-2,4-dion [Z-enol-(R,R,R)-34]

${ }^{1} \mathbf{H}$ NMR (500.10 MHz, $\left.\mathrm{CDCl}_{3}\right)$ :

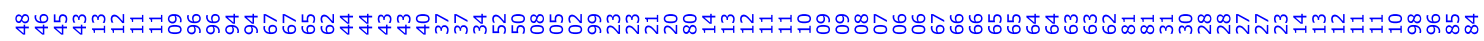

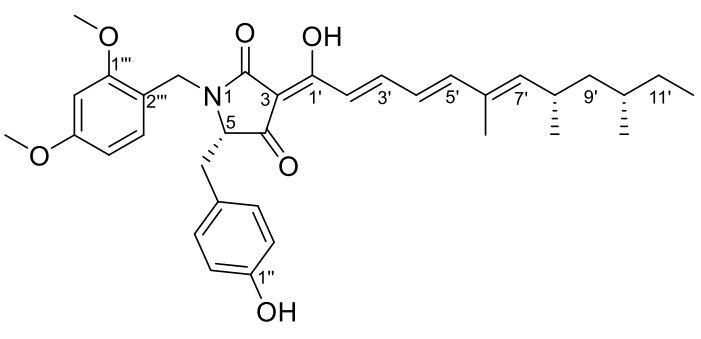

Z-enol-(S,S,S)-34

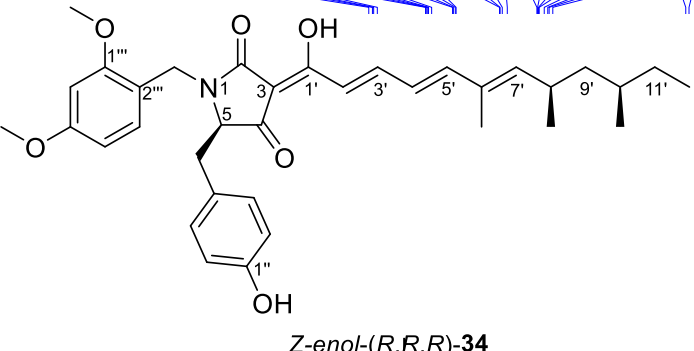

Z-enol-(R, $R, R)-34$ 
(5S)-5-(1'-Hydroxybenzyl)-1-(1'"',5'"'-dimethoxybenzyl)-3-(1'Z,2' $E, 4^{\prime} E, 6^{\prime} E, 8^{\prime} S, 10$ 'S)-1'-hydroxy-6',8',10'-trimethyldodeca-2',4',6'-trien-1'ylidene)pyrrolidine-2,4-dion [Z-enol-(S,S,S)-34]

and

(5R)-5-(1"'-Hydroxybenzyl)-1-(1'",5'"'-dimethoxybenzyl)-3-(1'Z,2' $\left.E, 4^{\prime} E, 6^{\prime} E, 8^{\prime} R, 10 ' R\right)-1^{\prime}$-hydroxy-6',8',10'-trimethyldodeca-2',4',6'-trien-1'ylidene)pyrrolidine-2,4-dion [Z-enol-(R,R,R)-34]

${ }^{13}$ C NMR (125.75 MHz, $\left.\mathrm{CDCl}_{3}\right)$ :

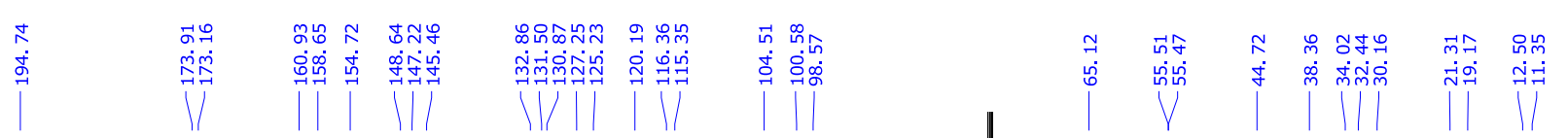

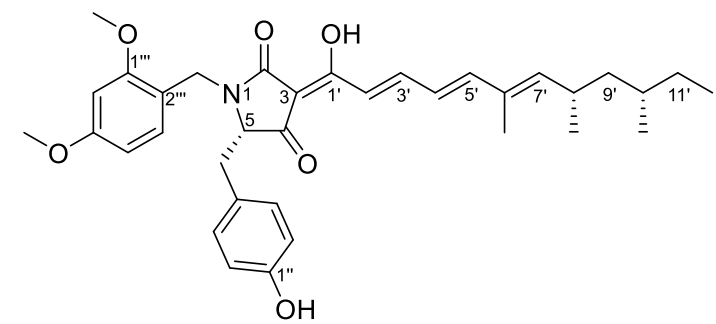

Z-enol-(S,S,S)-34

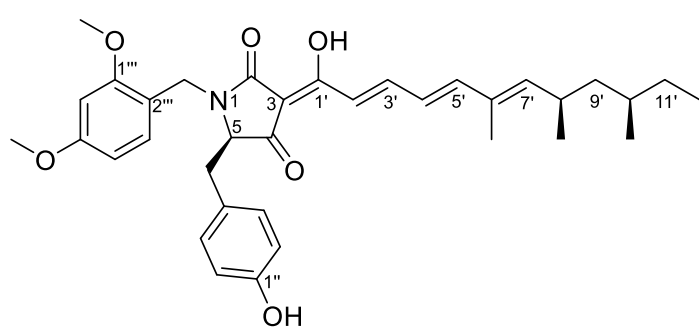

Z-enol-(R,R,R)-34 
(5S)-5-(1'-Hydroxybenzyl)-1-(1'",5'"'-dimethoxybenzyl)-3-(1'Z,2' $\left.E, 4^{\prime} E, 6^{\prime} E, 8^{\prime} R, 10 ' R\right)-1^{\prime}-$ hydroxy-6',8',10'-trimethyldodeca-2',4',6'-trien-1'ylidene)pyrrolidine-2,4-dion [Z-enol-(S,R,R)-34]

and

(5R)-5-(1"'-Hydroxybenzyl)-1-(1'"',5'"-dimethoxybenzyl)-3-(1'Z,2' $E, 4^{\prime} E, 6^{\prime} E, 8^{\prime} S, 10$ ' $\left.S\right)-1^{\prime}$-hydroxy-6', $8^{\prime}, 10^{\prime}$-trimethyldodeca-2',4',6'-trien-1'ylidene)pyrrolidine-2,4-dion [Z-enol-(R,S,S)-34]

${ }^{1} \mathbf{H}$ NMR $\left(500.10 \mathrm{MHz}, \mathrm{CDCl}_{3}\right)$ :

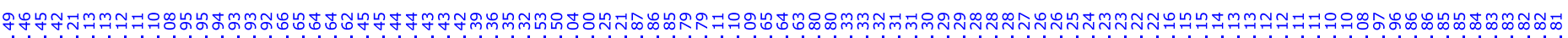

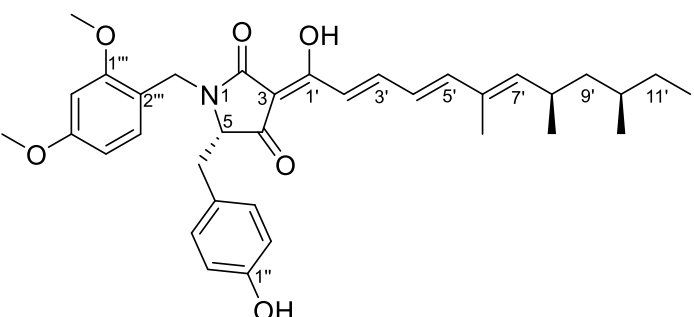

Z-enol-(S,R,R)-34

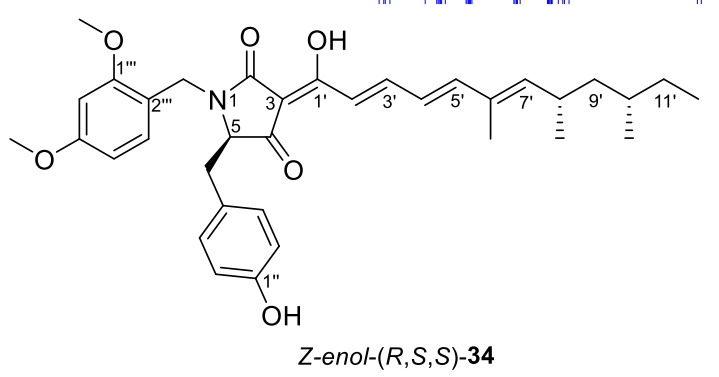

Z-enol-( $R, S, S)-\mathbf{3 4}$ 
(5S)-5-(1'-Hydroxybenzyl)-1-(1'",5'"'-dimethoxybenzyl)-3-(1'Z,2' $\left.E, 4^{\prime} E, 6^{\prime} E, 8^{\prime} R, 10 ' R\right)-1^{\prime}-$ hydroxy-6',8',10'-trimethyldodeca-2',4',6'-trien-1'ylidene)pyrrolidine-2,4-dion [Z-enol-(S,R,R)-34]

and

(5R)-5-(1"'-Hydroxybenzyl)-1-(1'"',5'"-dimethoxybenzyl)-3-(1'Z,2' $E, 4^{\prime} E, 6^{\prime} E, 8^{\prime} S, 10$ ' $\left.S\right)-1^{\prime}$-hydroxy-6', $8^{\prime}, 10^{\prime}$-trimethyldodeca-2',4',6'-trien-1'ylidene)pyrrolidine-2,4-dion [Z-enol-(R,S,S)-34]

${ }^{13}$ C NMR (125.75 MHz, $\left.\mathrm{CDCl}_{3}\right)$ :
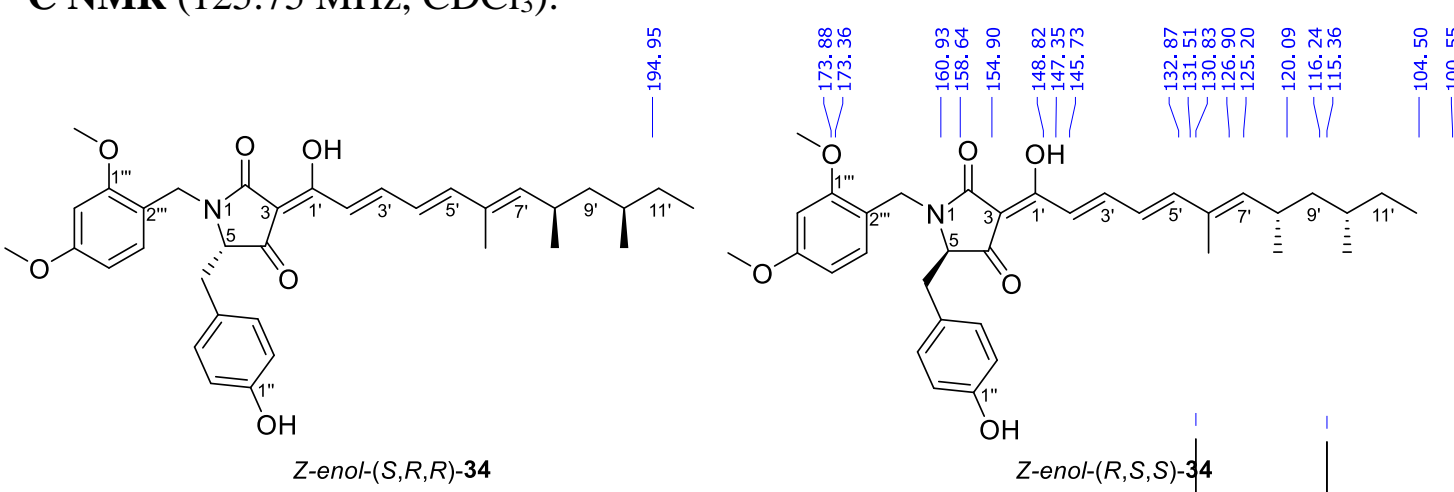

Z-enol-(S,R,R)-34

Z-enol-(R,S,S)-34 
(5S)-5-(1'-Hydroxybenzyl)-3-(1'Z,2' $\left.E, 4^{\prime} E, 6^{\prime} E, 8^{\prime} S, 10 ' S\right)-1^{\prime}$-hydroxy-6',8',10'-trimethyldodeca-2',4',6' -trien-1'-ylidene)pyrrolidine-2,4-dion [Z-enol-(S,S,S)-1]

and

(5R)-5-(1'-Hydroxybenzyl)-3-(1'Z,2' $\left.E, 4^{\prime} E, 6^{\prime} E, 8^{\prime} R, 10 ' R\right)-1$ '-hydroxy-6',8',10'-trimethyldodeca-2',4',6'-trien-1'-ylidene)pyrrolidine-2,4-dion [Z-enol- $(\boldsymbol{R}, \boldsymbol{R}, \boldsymbol{R})-1]$

${ }^{1}$ H NMR (500.10 MHz, MeOD):
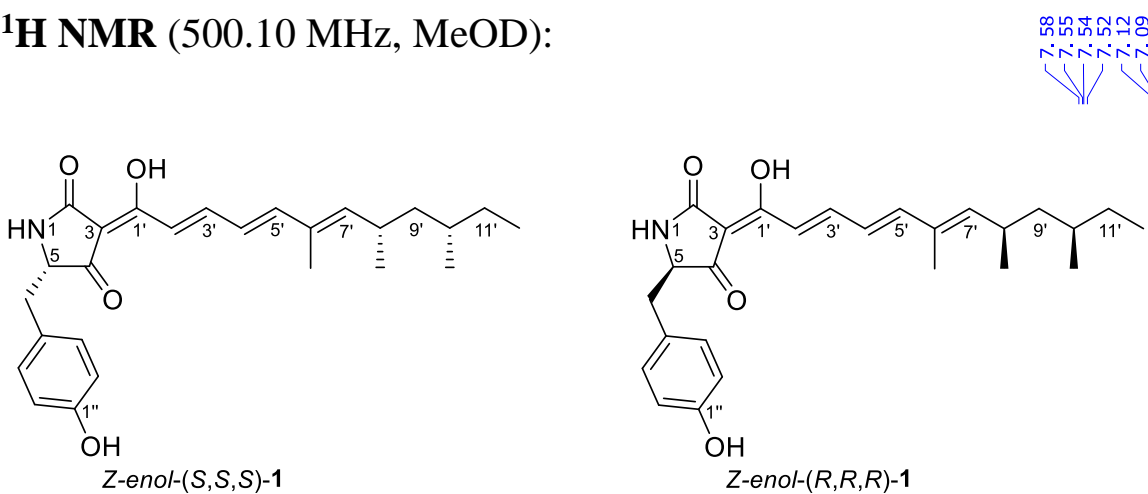

Z-enol-(R,R,R)-1

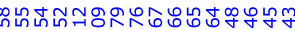

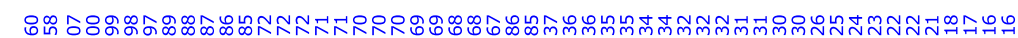

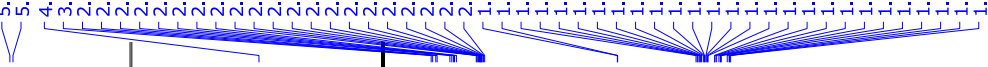

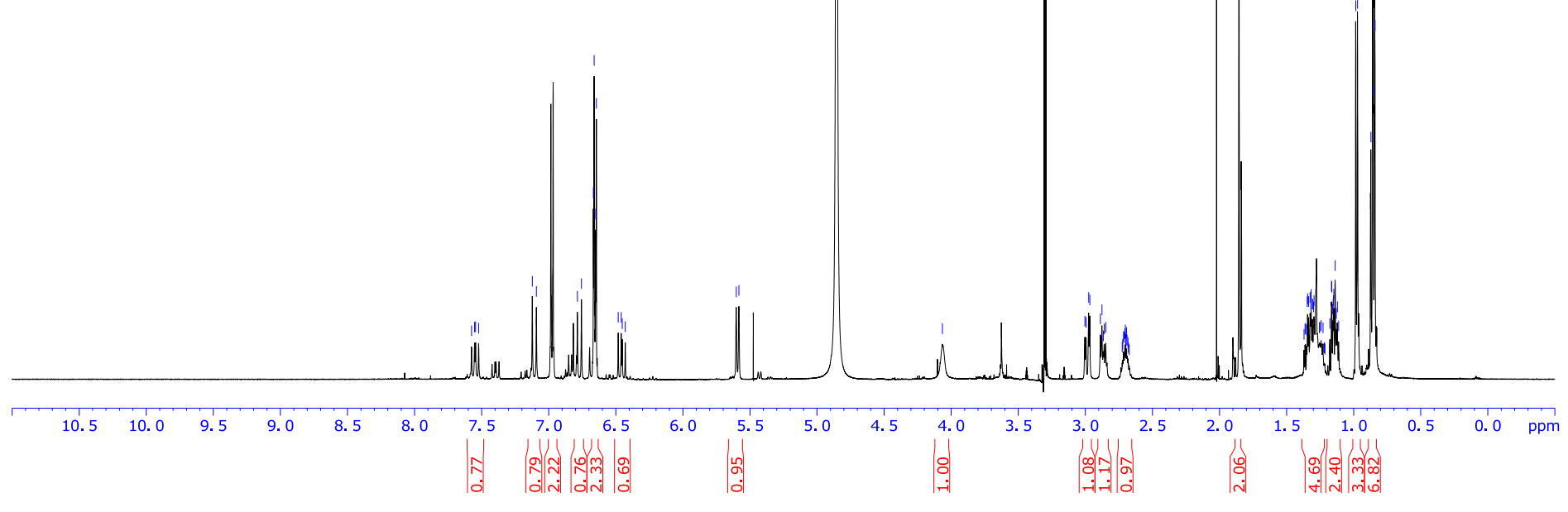


(5S)-5-(1'-Hydroxybenzyl)-3-(1'Z,2' $\left.E, 4^{\prime} E, 6^{\prime} E, 8^{\prime} S, 10 ' S\right)-1^{\prime}$-hydroxy-6',8',10'-trimethyldodeca-2',4',6'-trien-1'-ylidene)pyrrolidine-2,4-dion [Z-enol-(S,S,S)-1]

and

(5R)-5-(1'-Hydroxybenzyl)-3-(1'Z,2' $\left.E, 4^{\prime} E, 6^{\prime} E, 8^{\prime} R, 10 ' R\right)-1^{\prime}$-hydroxy-6',8',10'-trimethyldodeca-2',4',6'-trien-1'-ylidene)pyrrolidine-2,4-dion [Z-enol- $(R, R, R)-1]$

${ }^{13} \mathrm{C}$ NMR (125.75 MHz, MeOD):
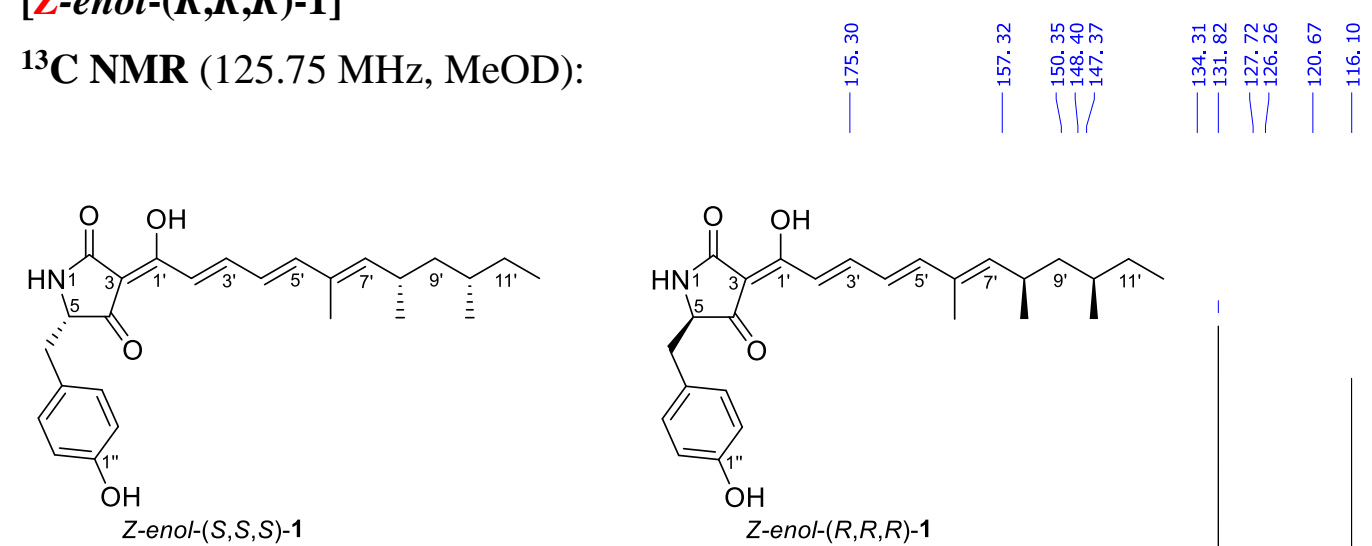

Z-enol-(S,S,S)-1

Z-enol-(R,R,R)-1

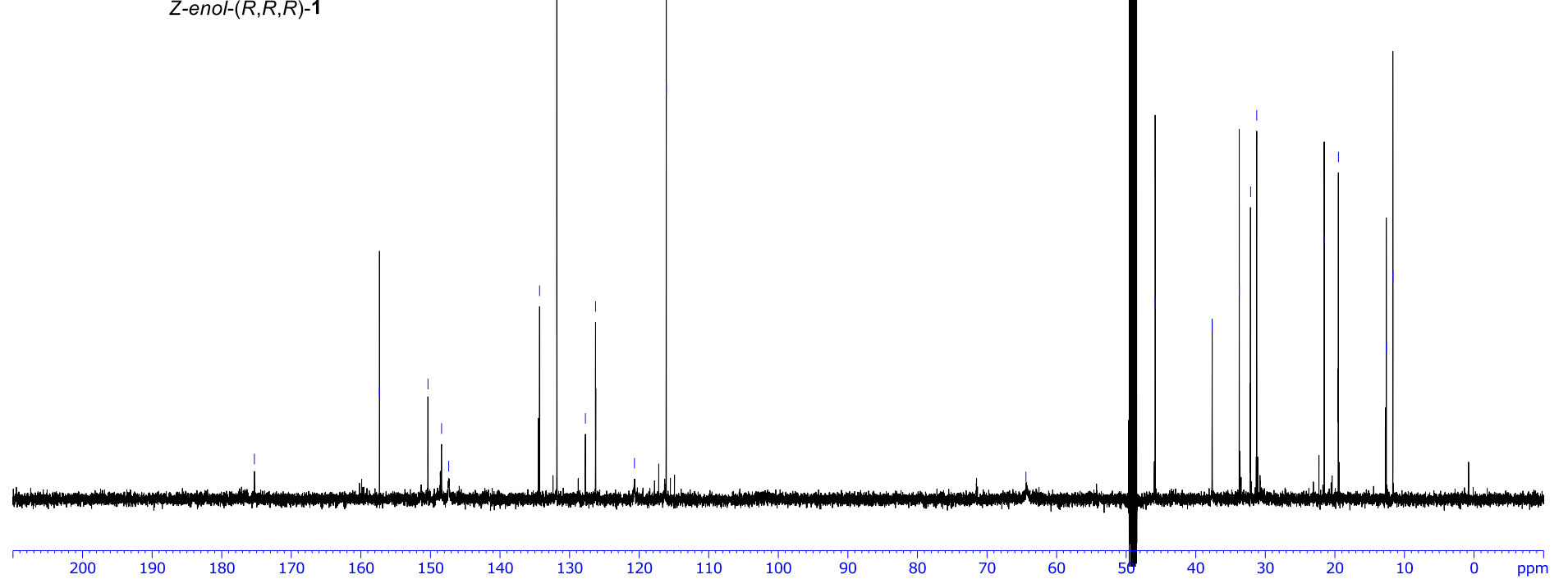


(5S)-5-(1'-Hydroxybenzyl)-3-(1'Z,2' $\left.E, 4^{\prime} E, 6^{\prime} E, 8^{\prime} R, 10 ' R\right)-1^{\prime}$-hydroxy-6',8',10'-trimethyldodeca-2',4',6'-trien-1'-ylidene)pyrrolidine-2,4-dion [Z-enol-(S,R,R)-1]

and

(5R)-5-(1'-Hydroxybenzyl)-3-(1'Z,2' $E, 4^{\prime} E, 6^{\prime} E, 8^{\prime} S, 10$ ' $\left.S\right)$-1'-hydroxy-6',8',10'-trimethyldodeca-2',4',6'-trien-1'-ylidene)pyrrolidine-2,4-dion [Z-enol-(R,S,S)-1]

${ }^{1} \mathbf{H}$ NMR (500.10 MHz, MeOD):
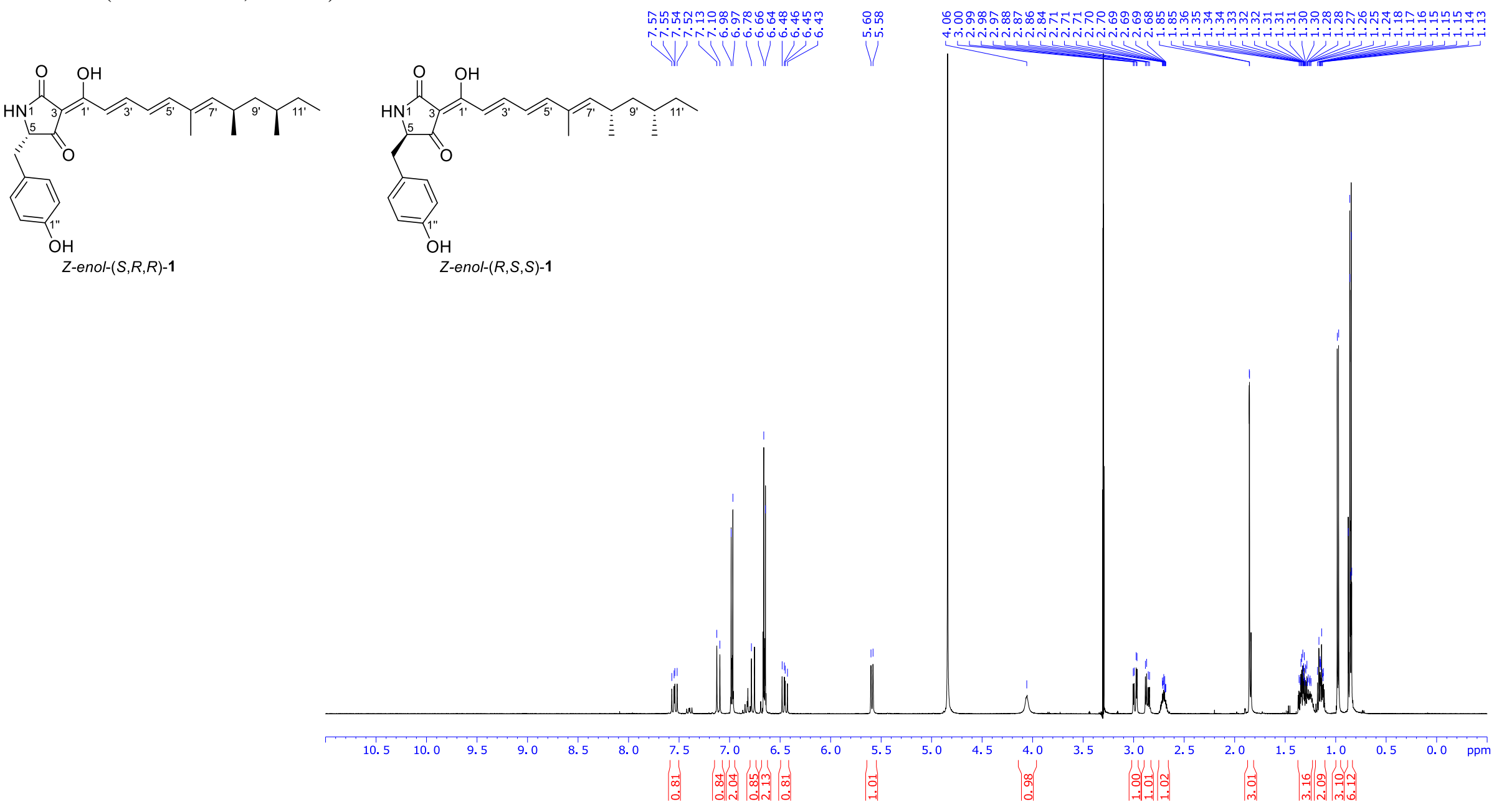
(5S)-5-(1''-Hydroxybenzyl)-3-(1'Z,2' $\left.E, 4^{\prime} E, 6^{\prime} E, 8^{\prime} R, 10 ' R\right)-1^{\prime}$-hydroxy-6',8',10'-trimethyldodeca-2',4',6'-trien-1'-ylidene)pyrrolidine-2,4-dion [Z-enol-(S,R,R)-1]

and

(5R)-5-(1'-Hydroxybenzyl)-3-(1'Z,2' $E, 4^{\prime} E, 6^{\prime} E, 8^{\prime} S, 10$ ' $\left.S\right)-1^{\prime}$-hydroxy-6',8',10'-trimethyldodeca-2',4',6'-trien-1'-ylidene)pyrrolidine-2,4-dion [Z-enol-(R,S,S)-1]

${ }^{13}$ C NMR (125.75 MHz, MeOD):
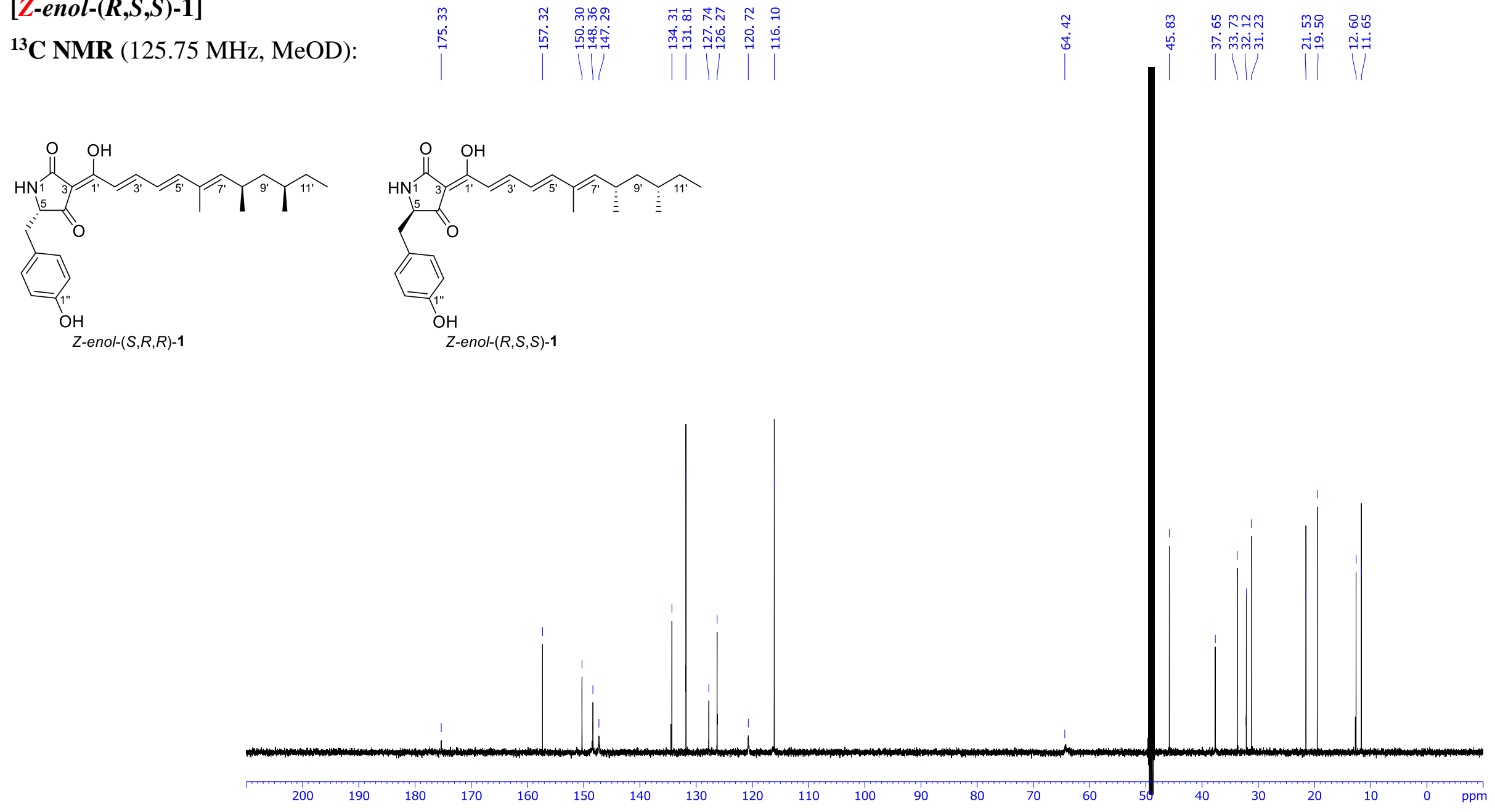
Christian Drescher, Morris Keller, Olivier Potterat, Matthias Hamburger and Reinhard Brückner, Structure-Elucidating Total Synthesis of the (Polyenoyl)tetramic Acid Militarinone C

3.4 Spectra of the Isolated Products of Paecilomyces militaris RCEF 0095 
(5S)-5-[1'"'-Hydroxy(1"'-hydroxybenzyl)]-3-(1'Z,2' $\left.E, 4^{\prime} E, 6^{\prime} E, 8^{\prime} R, 10^{\prime} R\right)-1^{\prime}$-hydroxy-6',8',10'-trimethyldodeca-2',4',6'-trien-1'ylidene)pyrrolidine-2,4-dion [Z-enol-(S,R,R)-2]

${ }^{1}$ H NMR (500.10 MHz, MeOD):

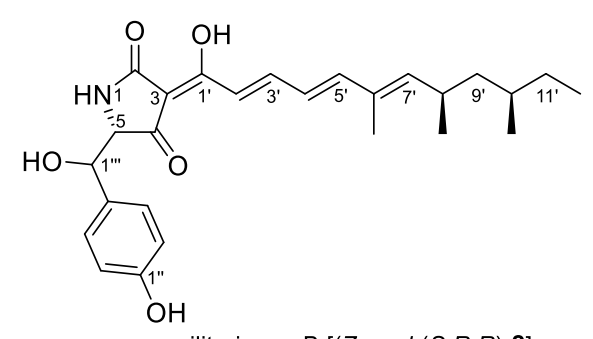

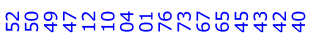

militarinone B $[(Z-e n o l-(S, R, R)-2]$

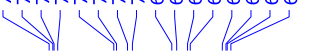

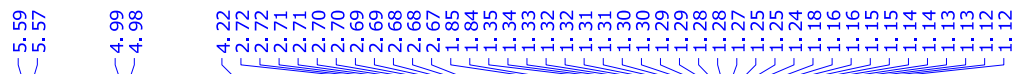

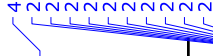

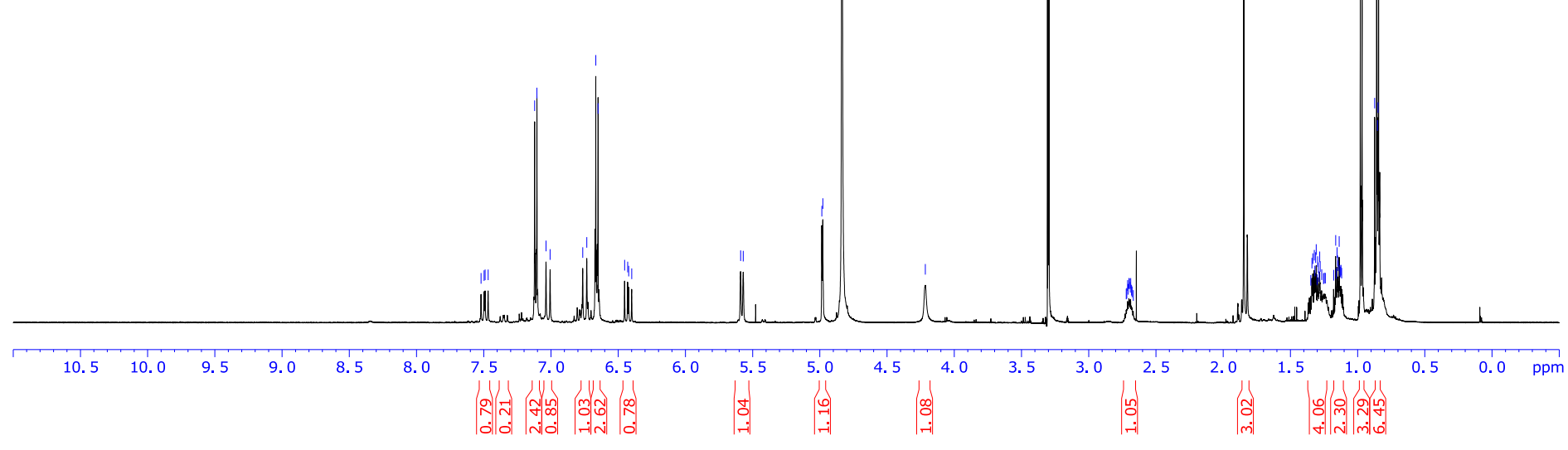


(5S)-5-[1'"'-Hydroxy(1"'-hydroxybenzyl)]-3-(1'Z,2' $\left.E, 4^{\prime} E, 6^{\prime} E, 8^{\prime} R, 10^{\prime} R\right)-1^{\prime}$-hydroxy-6',8',10'-trimethyldodeca-2',4',6'-trien-1'ylidene)pyrrolidine-2,4-dion [Z-enol- $(S, R, R)-2]$

${ }^{13}$ C NMR (125.75 MHz, MeOD):

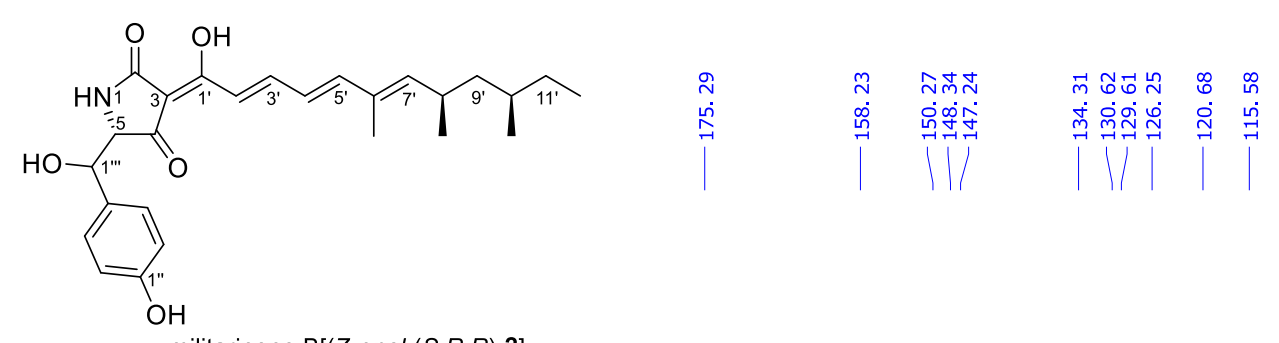

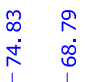

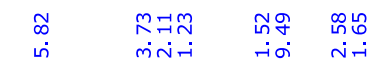

militarinone $\mathrm{B}[(Z-e n o l-(S, R, R)-2]$ 


\section{4-Hydroxy-5-(1"'-hydroxyphenyl)-3-[(2E,4E,6E,8R,10R)-6,8,10-trimethyldodeca-2,4,6-trienoyl]pyridin-2(1H)-one $[(R, R)-3]$}

${ }^{1}$ H NMR (500.10 MHz, MeOD):
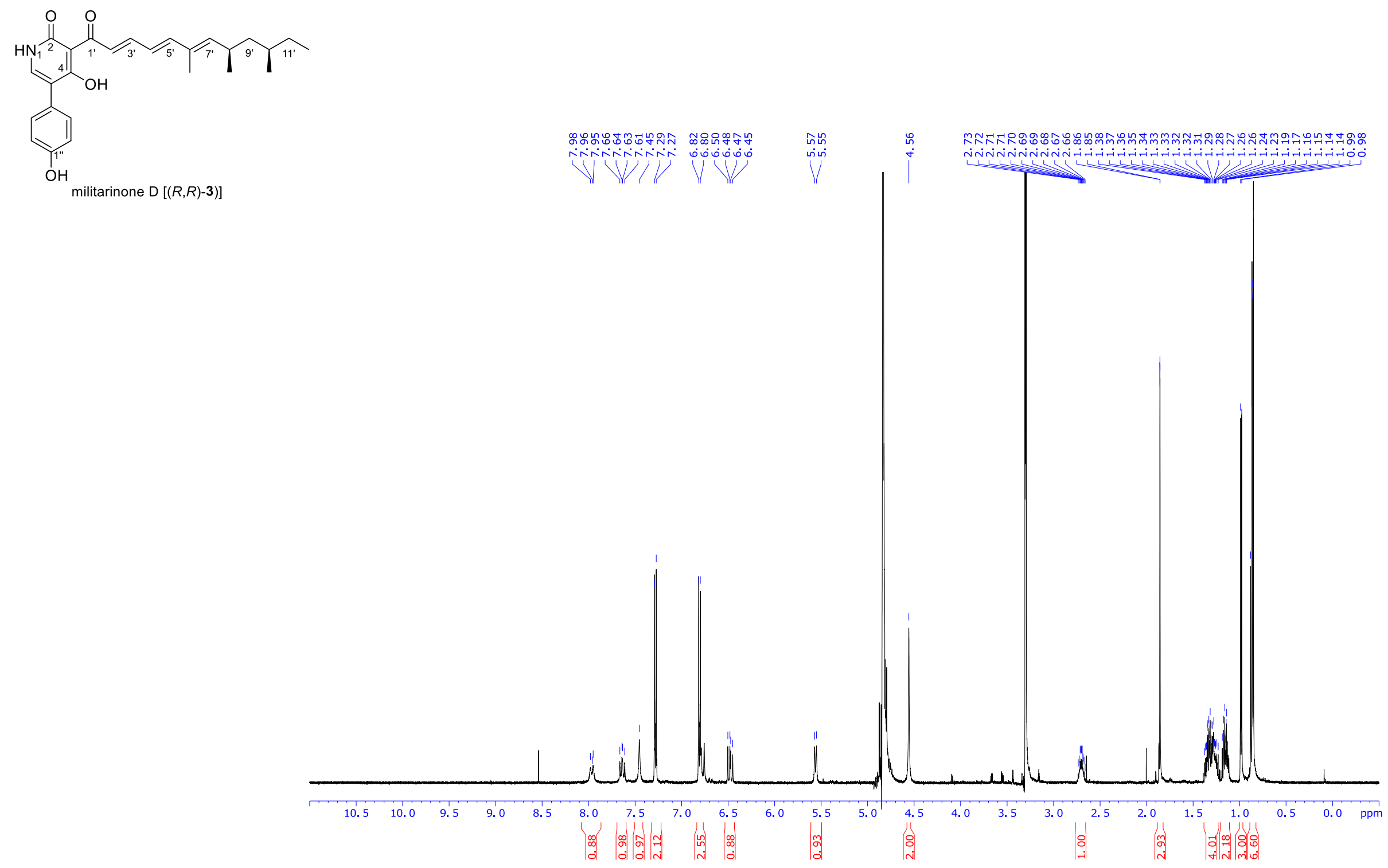
4-Hydroxy-5-(1"-hydroxyphenyl)-3-[(2E,4E,6E,8R,10R)-6,8,10-trimethyldodeca-2,4,6-trienoyl]pyridin-2(1H)-one $[(R, R)-3]$ ${ }^{13}$ C NMR (125.75 MHz, MeOD):
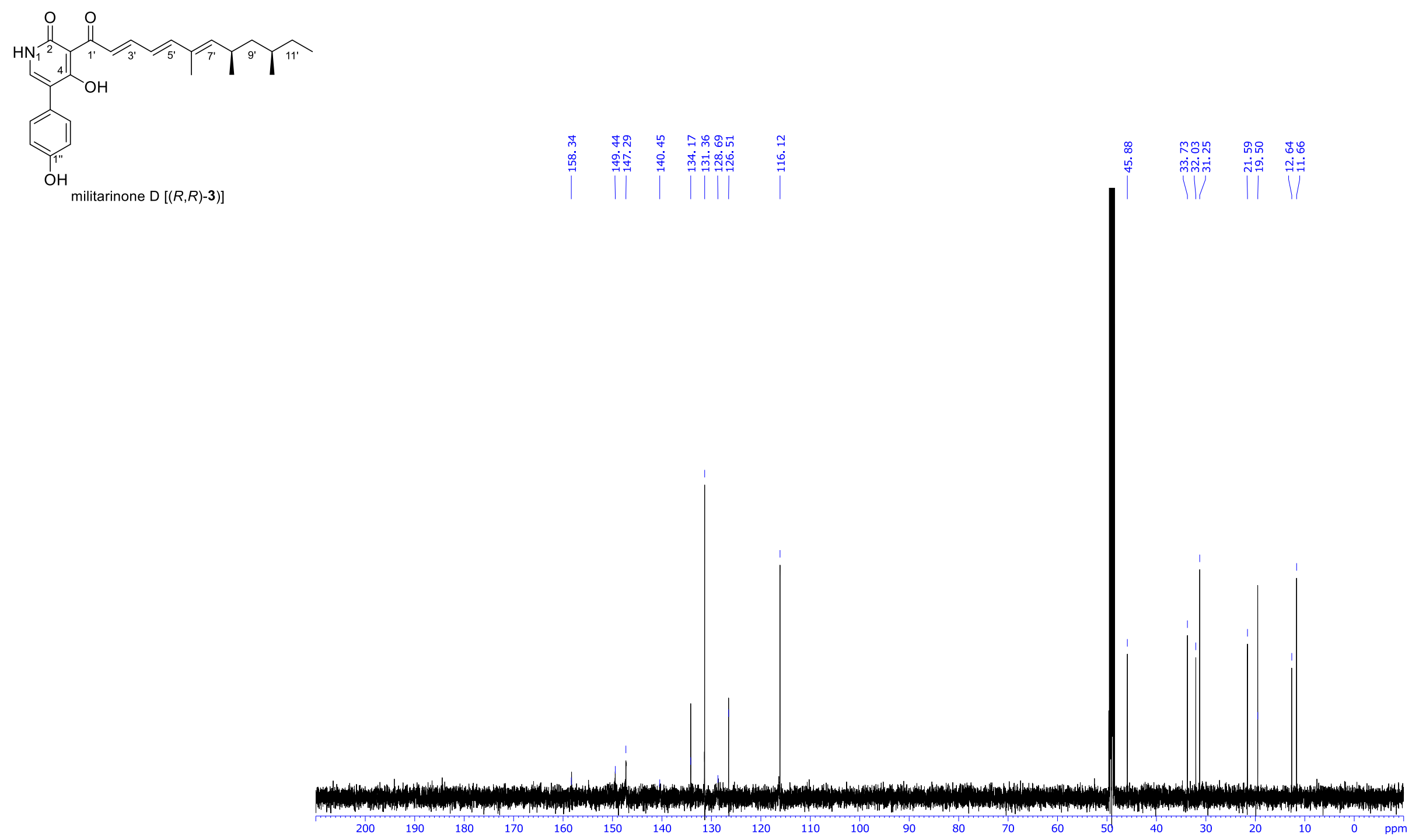
5-[(1"s,4"S)-1",4"-Dihydroxycyclohexyl]-4-hydroxy-3-[(2E,4E,6E,8R,10R)-6,8,10-trimethyldodeca-2,4,6-trienoyl]pyridin-2(1H)-one [(R,R4]

${ }^{1} \mathbf{H}$ NMR (500.10 MHz, MeOD):
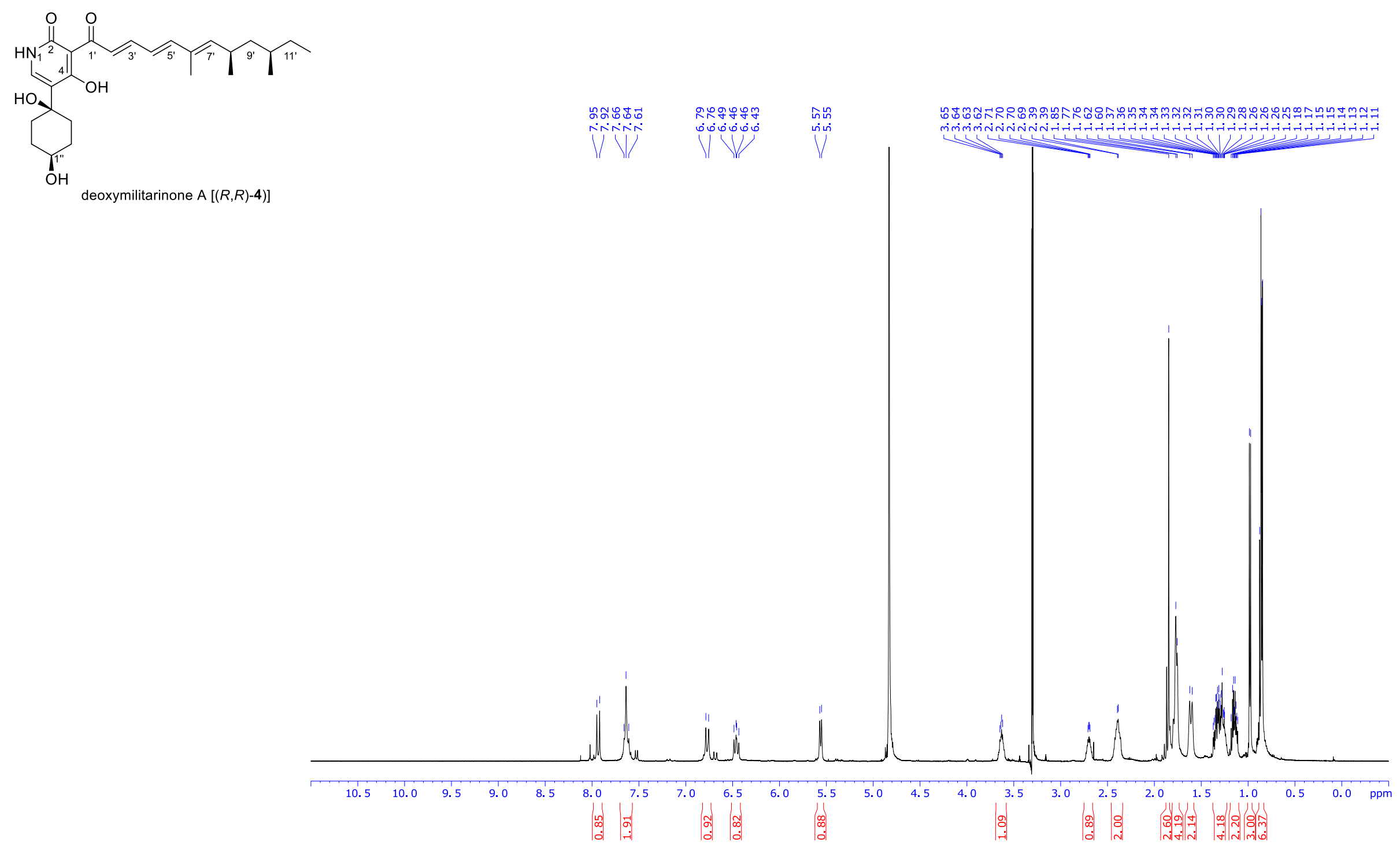
5-[(1"s,4"'S)-1",4"-Dihydroxycyclohexyl]-4-hydroxy-3-[(2E,4E,6E,8R,10R)-6,8,10-trimethyldodeca-2,4,6-trienoyl]pyridin-2(1H)-one [(R,R)4]

${ }^{13}$ C NMR (125.75 MHz, MeOD):

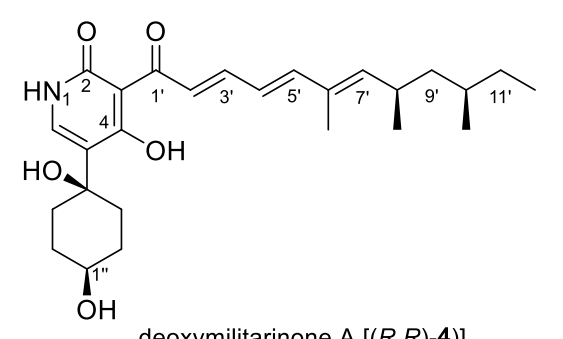

deoxymilitarinone $\mathrm{A}[(R, R)-4)]$ 


\section{5-[(1"s,4"'S)-1",4"-Dihydroxycyclohexyl]-1,4-dihydroxy-3-[(2E,4E,6E,8R,10R)-6,8,10-trimethyldodeca-2,4,6-trienoyl]pyridin-2(1H)-one} $[(\boldsymbol{R}, \boldsymbol{R})-5]$

${ }^{1}$ H NMR (500.10 MHz, MeOD):

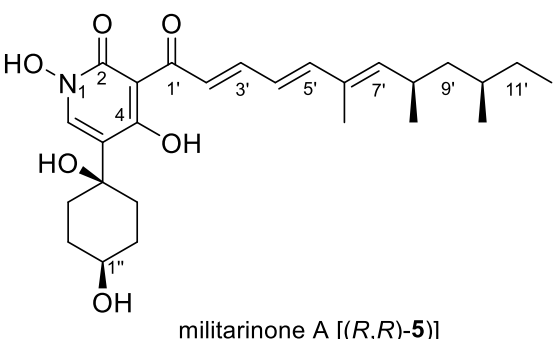

militarinone $\mathrm{A}[(R, R)-5)]$

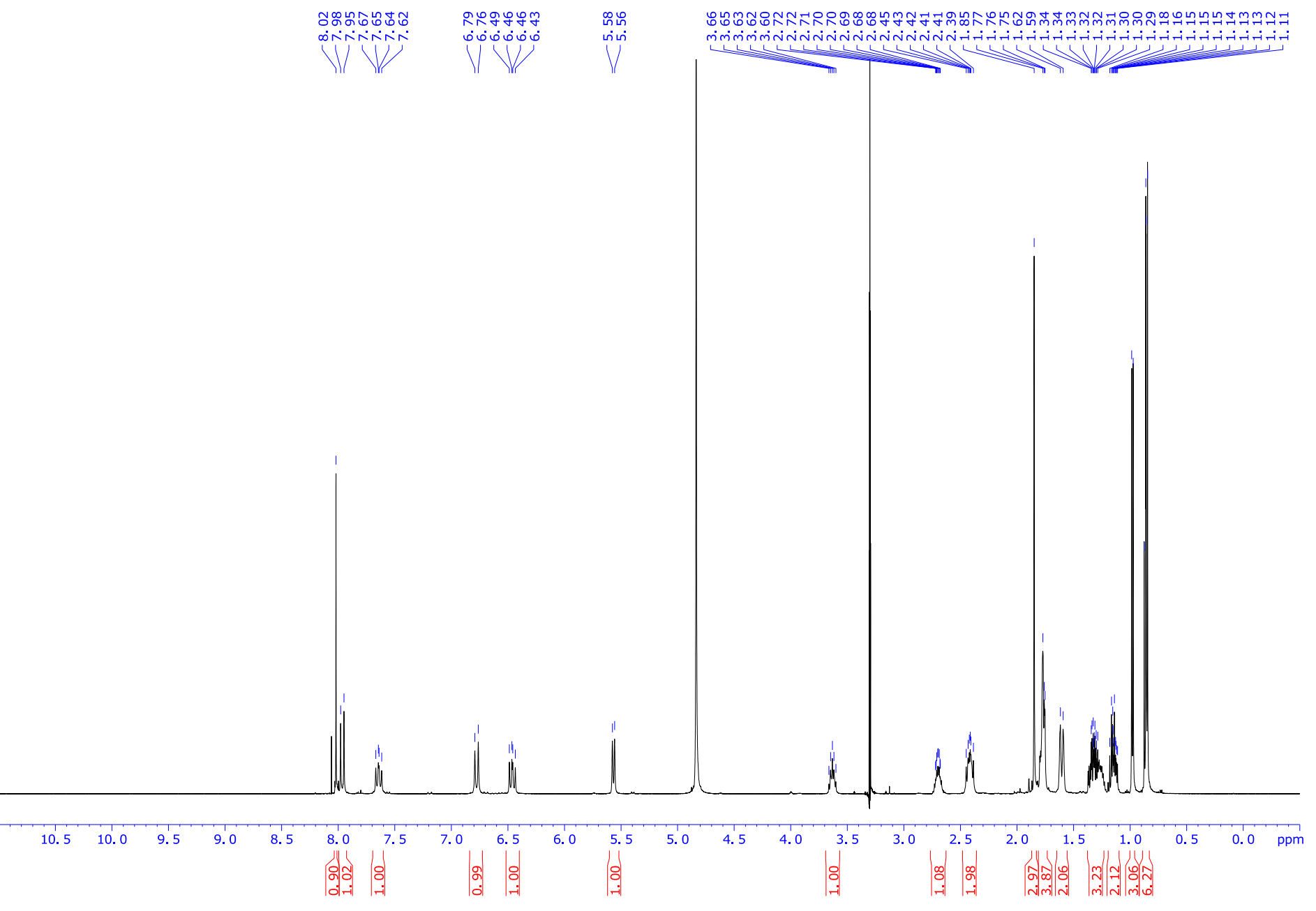


5-[(1"s,4"'S)-1",4"-Dihydroxycyclohexyl]-1,4-dihydroxy-3-[(2E,4E,6E,8R,10R)-6,8,10-trimethyldodeca-2,4,6-trienoyl]pyridin-2(1H)-one $[(\boldsymbol{R}, \boldsymbol{R})-5]$

${ }^{13}$ C NMR (125.75 MHz, MeOD):

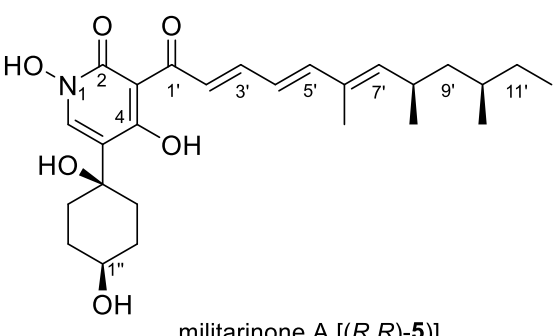

militarinone $\mathrm{A}[(R, R)-5)]$
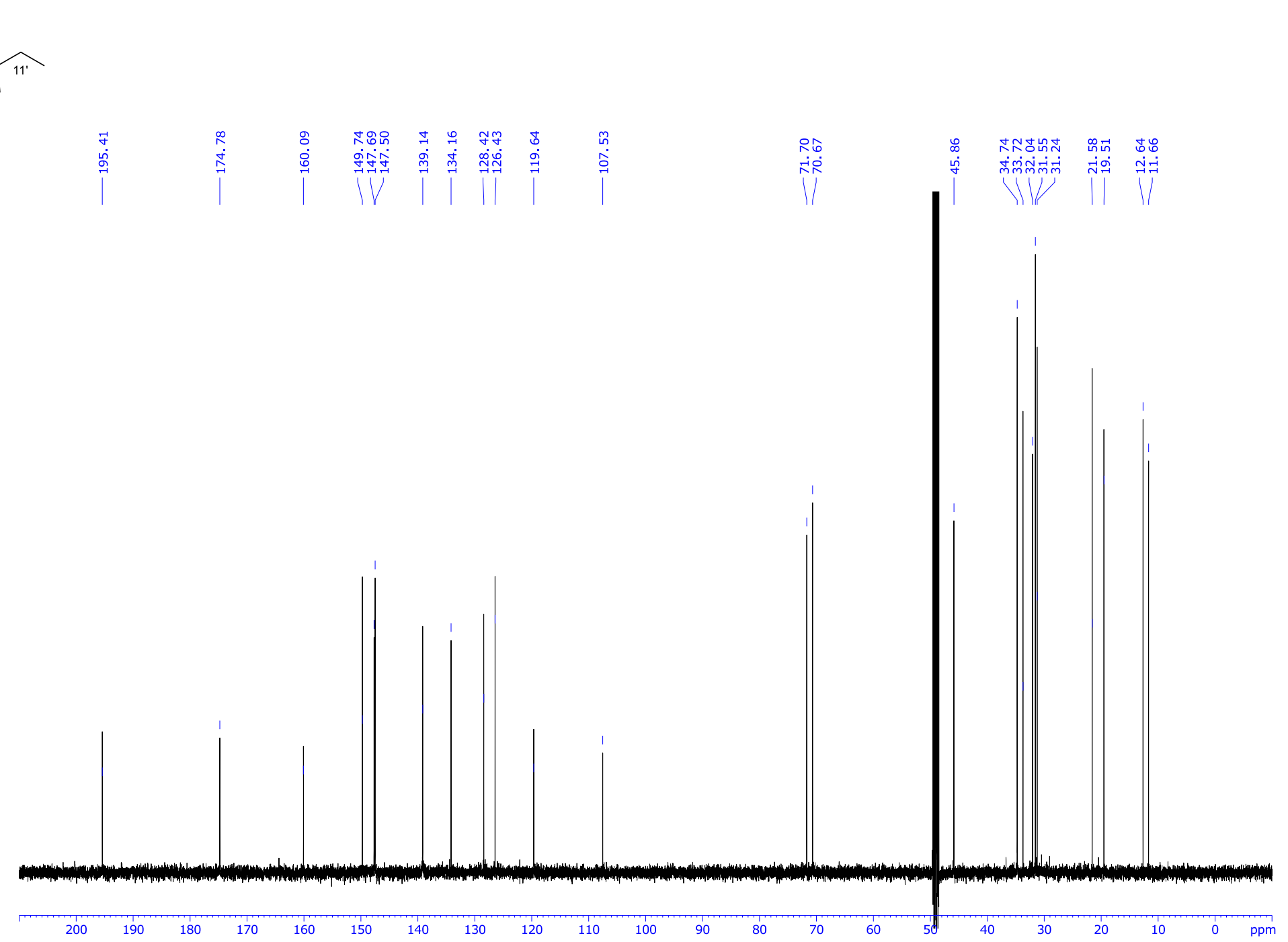


\section{$(4 R, 6 R, E)-2,4,6-T r i m e t h y l o c t-2-e n-1-o l ~[(R, R)-35]$}

and

\section{$(4 S, 6 S, E)-2,4,6-T r i m e t h y l o c t-2-e n-1-o l[(S, S)-35]$}

${ }^{1} \mathbf{H}$ NMR (300.13 MHz, $\left.\mathrm{CDCl}_{3}\right)$ :

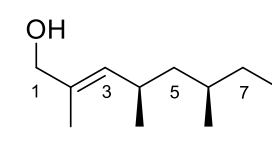

$(R, R)-35$
$\mathrm{OH}$

$$
\overbrace{(s, s)-35}
$$

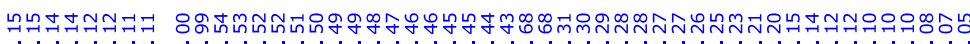

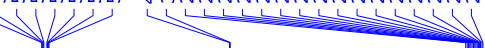

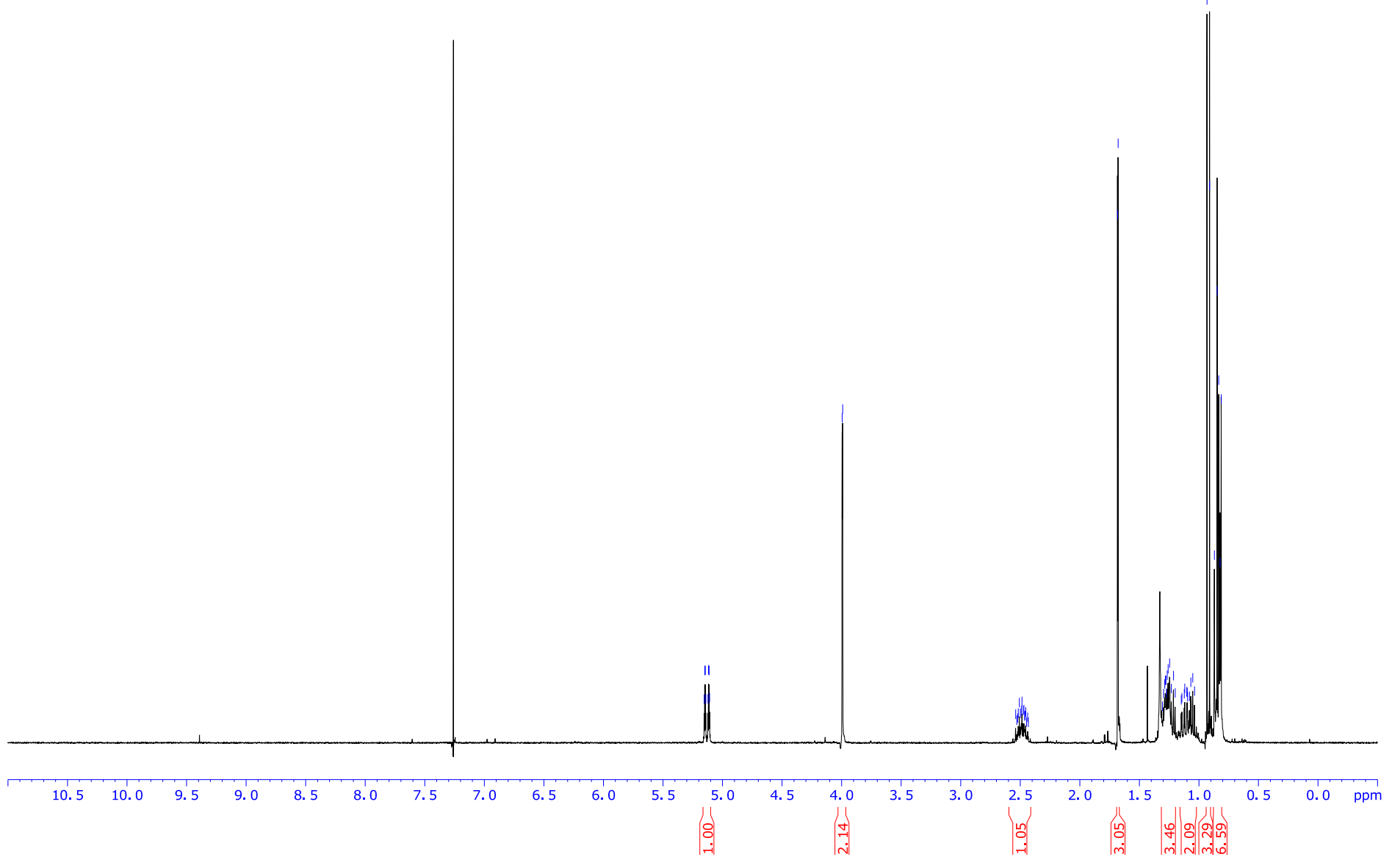

